\title{
Quantum Corrections to the Second Virial Coefficient for the Lennard-Jones (m-6) Potential
}

\author{
Marjorie E. Boyd \\ Institute for Basic Standards \\ National Bureau of Standards \\ Washington, D.C. 20234
}

(September 18, 1970)

\begin{abstract}
Tables are presented for the second virial coefficient of a Lennard-Jones $(m-6)$ gas in reduced variables for seven values of $\mathrm{m}$. A wide range of reduced temperatures, in a mesh chosen for ease of interpolation, is covered. Values are given for the classical term and three quantum corrections as well an the first two temperature derivatives of each of these terms. A discussion of the variation of behavior with $m$, especially at the limits $m=6$ and $m=\infty$, is included.
\end{abstract}

Key words: Lennard-Jones gas; $(m-6)$ intermolecular potential; quantum corrections; second virial coefficient; statistical mechanics; thermodynamics.

For the temperature range in which one is approaching classical behavior the second virial coefficient, $B$, for a Boltzmann gas may be represented by an asymptotic series in powers of $\hbar^{2}$. [1] ${ }^{1}$ That is, we can express $B$ as a classical term, $B^{(0)}$, plus quantum correction terms,

$$
B=B^{(0)}+q B^{(1)}+q^{2} B^{(2)}+\ldots .
$$

where $q=\hbar^{2} / 2 \mu, \quad(\hbar$ is Planck's constant divided by $2 \pi, \mu$ is the reduced mass of the molecule), and the $B^{(p)}$ are the quantum corrections of order $p$. Kihara, Midzuno and Shizume [2] give expressions for these terms up through third order as integrals over functions of the intermolecular potential and its derivatives.

This expansion of $B$ will not be applicable in cases where the intermolecular potential function or its derivatives contain discontinuities, as, for example, the hard sphere or square well cases. It has also been shown [3] that the exchange term of the second virial coefficient cannot be represented by such an expansion, so eq (1) will not be adequate for calculating $B$ at temperatures so low that spin and statistical effects are important. In practice, however, these temperatures will usually be below the range in which eq (1) converges.

At very low temperatures the second virial coefficient may be accurately calculated by means of the phase shift formulation, and we can examine the range of validity of eq (1) by comparing results obtained by both methods. In the case of $\mathrm{He}^{4}$, for example, it has been shown [3] that eq (1) with three quantum cor-

\footnotetext{
1 Figures in brackets indicate the literature references at the end of this paper.
}

rections gives results within 1 percent of the exact values down to almost $50 \mathrm{~K}$, while two quantum corrections are sufficient to give this accuracy above $70 \mathrm{~K}$. In general, results become much poorer as one approaches a temperature corresponding to the well depth, and eq (1) tends to become divergent below this temperature. For the more classical gases, such as the heavier rare gases, eq (1) should give useful values down to nearly their melting temperatures.

If we introduce the reduced quantities:

$$
\begin{aligned}
B^{*} & =B /\left(b_{0}\right) \\
U^{*} & =U / \epsilon \\
T^{*} & =h T / \epsilon \\
R & =r / \sigma \\
\Lambda^{*} & =h / \sigma \sqrt{2 \mu \epsilon}
\end{aligned}
$$

where $b_{0}=2 / 3 \pi N \sigma^{3}$ is the classical hard sphere second virial coefficient, $U$ is the intermolecular potential, $\epsilon$ is the depth of the potential well, $T$ is the temperature, $k$ is Boltzmann's constant, and $\sigma$ is the molecular diameter, we can write eq (1) as:

$$
B^{*}=B^{*(0)}+\Lambda^{* 2} B^{*(1)}+\Lambda^{* 4} B^{*(2)}+\ldots .
$$

where the Kihara $B^{(p)}$ 's become:

$B^{*(0)}=-3 \int_{0}^{\infty}\left(e^{-l * / T *}-1\right) R^{2} d R$

$B^{*(1)}=\frac{1}{16 \pi^{2} T^{* 3}} \int_{0}^{\infty} e^{-l^{*} * / T^{*}}\left(U^{* \prime}\right)^{2} R^{2} d R$ 


$$
\begin{aligned}
B^{*(2)}= & -\frac{1}{4\left(4 \pi^{2}\right)^{2} T^{* 4}} \int_{0}^{\infty} e^{-U^{* / T} *}\left[\frac{\left(U^{* \prime \prime}\right)^{2}}{10}+\frac{\left(U^{* \prime}\right)^{2}}{4 R^{2}}\right. \\
& \left.+\frac{\left(U^{* \prime}\right)^{3}}{9 T^{*}}-\frac{\left(U^{* \prime}\right)^{4}}{72\left(T^{*}\right)^{2}}\right] R^{2 \cdot} d R \\
B^{*(3)=}= & \frac{3}{2\left(4 \pi^{2}\right)^{3} T^{* 5}} \int_{0}^{\infty} e^{-l^{*} / T^{*}}\left[\frac{\left(U^{* \prime \prime \prime}\right)^{2}}{840}+\frac{\left(U^{* \prime \prime}\right)^{2}}{140 R^{2}}\right. \\
& +\frac{\left(U^{* \prime \prime}\right)^{3}}{756 T^{*}}+\frac{U^{* \prime}\left(U^{* \prime \prime}\right)^{2}}{180 T^{*} R}+\frac{\left(U^{* \prime}\right)^{3}}{945 T^{*} R^{3}} \\
& -\frac{\left(U^{* \prime}\right)^{2}\left(U^{* \prime \prime}\right)^{2}}{720\left(T^{*}\right)^{2}}-\frac{\left(U^{* \prime}\right)^{4}}{6480\left(T^{*}\right)^{2} R^{2}} \\
& \left.-\frac{\left(U^{* \prime}\right)^{5}}{2160\left(T^{*}\right)^{3} R}+\frac{\left(U^{* \prime}\right)^{6}}{25920\left(T^{*}\right)^{4}}\right] R^{22} d R .
\end{aligned}
$$

For the case of the general Lennard-Jones $(m-6)$ potential in reduced variables,

$$
U^{*}(r)=\frac{\left[R^{-m}-R^{-6}\right]}{C(m, 6)}
$$

where:

$$
C(m, 6)=\left(\frac{6}{m}\right)^{\frac{6}{m-6}}\left(1-\frac{6}{m}\right)
$$

it has been shown [4] that when the part of the exponential containing the $R^{-6}$ term is expanded in an infinite series, these expressions can be integrated term by term, giving power series. The resulting expressions for $B$ through third order are given by Kihara et al. [2] and, when translated into reduced variables, are:

$$
\begin{aligned}
B^{*(p)}= & \frac{(-)^{p+1} \cdot 3}{(2 \pi)^{2 p}} \sum_{t=0}^{\infty} T^{*\left[\left(\frac{6}{m}-1\right) t-p-\frac{p^{\prime}}{m}\right]} \Gamma\left(\frac{6 t-p^{\prime}}{m}\right) \frac{1}{t !} \\
& \times \frac{m^{\left[t-1+\frac{p^{\prime}}{m-6}\right]} \cdot J_{t}^{(p)}(6, m)}{(m-6)^{\left[\left(1-\frac{6}{m}\right) t+\frac{p^{\prime}}{m}\right]} \cdot 6^{\left[\frac{6}{m} t-\frac{p^{\prime}}{m}+\frac{p^{\prime}}{m-6}\right]}}
\end{aligned}
$$

where $p^{\prime}=3-2 p$, and

$$
\begin{aligned}
J_{t}^{(0)}= & 1 . \\
J_{t}^{(1)}= & {[(m-6) 6 t-(m-1)] / 12 . } \\
J_{t}^{(2)}= & {\left[21(m-6)^{2} 6^{2} t^{2}+\left(6 m^{2}+30 m-42\right)(m-6) 6 t\right.} \\
& \left.+6 m^{3}-27 m^{2}+30 m+63\right] / 4320 . \\
J_{t}^{(3)}= & {\left[93(m-6)^{3} 6^{3} t^{3}+\left(82 m^{2}+657 m-741\right)\right.} \\
& (m-6)^{2} 6^{2} t^{2} \\
+ & \left(24 m^{4}+348 m^{3}+93 m^{2}-810 m+2241\right)(m-6) 6 t \\
+ & 72 m^{5}-126 m^{4}-531 m^{3}+1755 m^{2}-405 m \\
& -3645] / 362880 .
\end{aligned}
$$

Since these corrections find wide applicability for Lennard-Jones $(m-6)$ type potentials, we have prepared numerical tables for a family of values of $m(6,9,12,15,18,24,36)$ over a wide range of values of $T^{*}$ for which eq (3) is useful. The first two temperature derivatives,

$$
T^{*} \partial B^{(p)} / \partial T^{*} \quad \text { and } \quad\left(T^{*}\right)^{2} \frac{\partial^{2} B^{(p)}}{\partial T^{* 2}},
$$

which are necessary for the calculation of thermodynamic quantities and sound velocities, are also given. They were obtained by differentiating eqs (4) with respect to $T^{*}$ and numerically integrating the resulting expressions.

These tables were calculated by Simpson numerical integration of eqs (4) from a value of $R=R_{s}$ below which the exponential becomes negligibly small. The contribution to the classical term is then simply $R_{s}^{3}$ while this part of the integral gives a vanishingly small contribution to the quantum corrections and derivatives. The numerical integration is carried out from $R_{s}$ to $R=10$, and the contributions from the large $R$ region are obtained by expanding the exponential and integrating analytically a sufficient number of terms to give the desired numerical accuracy. The resulting values were tested for accuracy by decreasing the interval size used in the integration until no changes resulted. For the $(6-6)$ and $(9-6)$ the value $1 / 128$ was sufficient, for (12-6) through (24-6), 1/256 was used, and for the $(36-6), 1 / 320$ was required. Higher order integration procedures than Simpson were also tried and found to be unnecessary. The numbers given in the tables are believed to be correct to within 1 in the last digit given.

$B^{*(0)}$ and its temperature derivatives could be checked against previous calculations for several of the potentials. For the (9-6) Epstein and Hibbert [5] give values for a number of $T^{*}$ 's ranging from 0.5 to 100 calculated by means of the series expansions (eq 6). Bird and Spotz [6] give results obtained in the same way for the (12-6) for $T^{*}$ 's covering our complete range, and Michels [7] also gives (12-6) results at low $T^{*}$ values (below 10), including the first two quantum corrections, obtained in terms of confluent hypergeometric functions. In all cases our results were in complete agreement to all figures available.

For the (18-6) Saxena and Joshi [8] calculated values for $B^{*(0)}$ and its first derivative from the series, but in terms of $T_{S . J}^{*}=(8 / 3 \sqrt{3}) T^{*}$, so comparisons are somewhat difficult. Interpolating in our tables for a few values over their range $\left(T_{S, J}^{*}=0.5\right.$ to 400$)$ indicates approximate agreement. Values of $B^{*(0)}$ for a large number of values of $m$ have been given by Sze and Hsu [9] but the formula they evaluated is incorrect except for $m=12$. For this case results are also in agreement. Spot checks of values for the other potentials were made by comparison with values calculated by means of eqs (6).

The $T^{*}$ intervals have been chosen such that fourpoint Lagrangian interpolation will suffice to give 
values correct to within 5 in the sixth digit given throughout the table, with the exception of -ome $T^{*}$ 's $<1$, and some higher order correction terms which have very small values and are given to fewer digits. We have included only a few small values of $T^{*}$ as eq (1) is convergent here only for very small values of $\Lambda^{*}$, i.e. nearly classical systems.

In considering the behavior of $B^{*}$ with $m$, it is useful to refer to figure 1 which shows the reduced potential

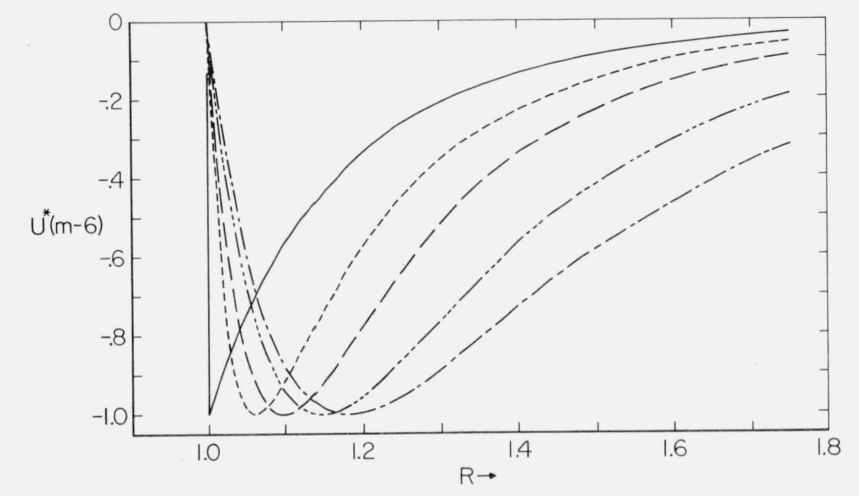

FIGURE 1. Lennard-Jones $(\mathrm{m}-6)$ potential, $\mathrm{U}^{*}(\mathrm{~m}-6)$, for various values of $\mathrm{m}:-, \mathrm{m}=\infty ;---, \mathrm{m}=36 ;--\longrightarrow, \mathrm{m}=18 ;----$, $\mathrm{m}=9 ;---, \mathrm{m}=6$.

function for selected values of $m$ includin $\%$ the limiting cases $m=6$ and $m=\infty$. One notes that as $m$ increases, the well becomes narrower and the strength of the well can thus be considered as weaker. As a consequence, the reduced Boyle temperature (where the repulsive effects cancel the attractive effects and $B^{*}$ becomes zero) becomes lower as $m$ increases. (It can also be noted from eq (4) that the first quantum correction is always positive and thus the more quantum mechanical the system, the more the Boyle temperature is lowered from the classical value.)

At higher $T^{*}$ 's the repulsive effect, which predominates, is eventually dimmed somewhat by core penetration, as was pointed out by Lennard-Jones, and $B^{*}$ passes through a maximum and then falls off as the system sees a smaller and smaller effective core size. This decrease is smaller the larger the $m$ value, and the drop-off becomes less and less, until for $m=\infty$, no maximum occurs. The temperature at which the maximum occurs $\left(T_{\max }^{*}\right)$ is the result of a competition between the rise in $B^{*}$ due to the loss of attractive effects from the declining strength of the well, and the drop in $B^{*}$ due to decreasing effective core size. Thus $T_{\max }^{*}$ as a function of $m$ passes through a minimum for $m$ near 18.

Examination of the limiting cases is of interest to illustrate the caution which must be exercised in inferring the behavior of $B$ in dimensioned units with $m$ from that of $B^{*}$.
If we consider the most general Lennard-Jones potential in the usual dimensioned coordinates

$$
U(r)=\lambda r^{-m}-\mu r^{-n}
$$

it is apparent that a nonvanishing well appears at a finite $r$ only if $m>n$. As $m \rightarrow n, U(r)$ becomes purely attractive or repulsive depending on the relative values of $\lambda$ and $\mu$, and it vanishes identically when they are equal. If we now change over into a representation in terms of $(\epsilon)$, the magnitude of the potential at the location where its derivative is zero, and $(\sigma)$, the location of the zero of the potential, instead of $\lambda$ and $\mu$, we obtain

where

$$
U(r)=\frac{\epsilon\left[\left(\frac{\sigma}{r}\right)^{-m}-\left(\frac{\sigma}{r}\right)^{-n}\right]}{C_{m, n}}
$$

$$
C_{m, n}=\left(\frac{n}{m}\right)^{\frac{n}{m-n}}\left(1-\frac{n}{m}\right) .
$$

In terms of reduced variables this becomes

$$
U^{*}(R)=\frac{R^{-m}-R^{-n}}{C_{m, n}}
$$

which, when $n=6$, becomes eq (5).

Now, however, because of the variables chosen, eq (10) will always contain a well at a finite $R$. If $m<n$, it is apparent from eq (9) that the coefficient changes sign and thus the larger exponent is always on the repulsive term. When $m \rightarrow n$, eq (10) approaches the limiting form

$$
U^{*}(R)=-n e R^{-n} \ln R
$$

which has a zero at $R=1$ and a minimum at $R=e^{1 / n}$.

For this limiting case it must be noted that the variables $\sigma$ and $\epsilon$ are no longer appropriate quantities for parameterizing the potential function as, when $m \rightarrow n, \sigma$ becomes infinite or indeterminate and $\epsilon$ is zero. Equation (11) cannot distinguish between the vanishing and purely attractive or repulsive limits of $U$ having lost the information about $\lambda$ and $\mu$.

When $B^{*}\left(T^{*}\right)$ is calculated for the potential given by eq (11), it has the usual form, beginning at negative values for low $T^{*}$ and becoming positive at higher $T^{*}$ s. Because of the loss of physical information in the potential function, however, these all correspond to $T=0$ and indeterminate $B$ 's. Thus these values, which we include in our tables, should be regarded as useful only as limiting values for interpolating with respect to $m$ and one must keep in mind the peculiar nature of the limit.

When $m \rightarrow \infty$, eq (10) becomes

$$
\begin{aligned}
U^{*}(R) & =-R^{-n} & \text { for } R & >1 \\
& =\infty & R & <1
\end{aligned}
$$

which is the so-called Sutherland potential. The $B^{*(0)}$ 
has been calculated in this case by Sherwood and Prausnitz [10] over a range of $T^{*}$ from 0.3 to 10 , again by series expansion. The expressions for the quantum corrections given here blow up as the potential is no longer analytic and the expansion in eq (1) does not hold.

Interpolating with respect to $m$ in these tables is more difficult for smaller $m$ values since $B^{*}$ changes more rapidly with $m$ as $m \rightarrow 6$. For large $m$ values it is necessary to use more widely spaced $m$ values if one wishes to interpolate with equal intervals, but $B$ is more slowly changing. Roughly, for low $T^{*}$ 's, about 3 digits can be expected below $m=9$ with 4 point Lag- range interpolation and for $m$ between 9 and 18 with 3 point interpolation. At high $T^{*}$ s (above the Boyle temperature) these same interpolation schemes would give about 4 digits.

A computer program giving the quantum corrections to $B$ for the (12-6) potential was prepared and tested by S. Y. Larsen with the assistance of the author [3] and served as a starting point for the more generalized program used here. We also wish to thank M. Klein for use of a differencing program to examine the mesh size needed for interpolation as well as encouragement and support of these calculations. 
$(m=6)$

\begin{tabular}{|c|c|c|c|c|c|c|c|c|c|c|c|c|}
\hline $\mathrm{T}^{*}$ & $\mathrm{~B}^{*(0)}$ & $\mathrm{B}^{*(1)}$ & $\mathrm{B}^{*(2)}$ & $\mathrm{B}^{*(3)}$ & $\mathrm{T}^{*} \frac{\partial \mathrm{B}^{*(0)}}{\partial \mathrm{T}^{*}}$ & $\mathrm{~T}^{*} \frac{\partial \mathrm{B}^{*(1)}}{\partial \mathrm{T}^{*}}$ & $\mathrm{~T}^{*} \frac{\partial \mathbf{B}^{*(2)}}{\partial \mathrm{T}^{*}}$ & $\mathrm{~T}^{*} \frac{\partial \mathrm{B}^{*(3)}}{\partial \mathrm{T}^{*}}$ & $T^{* 2} \frac{\partial^{2} B^{*(0)}}{\partial T^{* 2}}$ & $\mathrm{~T}^{* 2} \frac{\partial^{2} \mathrm{~B}^{*(1)}}{\partial \mathrm{T}^{* 2}}$ & $\mathrm{~T}^{* 2} \frac{\partial^{2} \mathrm{~B}^{*(2)}}{\partial \mathrm{T}^{* 2}}$ & $\mathrm{~T}^{* 2} \frac{\partial^{2} \mathrm{~B}^{*(3)}}{\partial \mathrm{T}^{* 2}}$ \\
\hline $\begin{array}{l}.300 \\
.400 \\
.500 \\
.600 \\
.700\end{array}$ & $\begin{array}{l}-.5200306+02 \\
-.2687889+02 \\
-.1760689+02 \\
-.1292147+02 \\
-.1012043+02\end{array}$ & $\begin{array}{l}.1808918+02 \\
.5034017+01 \\
.2166802+01 \\
.1177066+01 \\
.7362933+00\end{array}$ & \begin{tabular}{|l|}
$-.1083039+02$ \\
$-.1862926+01$ \\
$-.5573014+00$ \\
$-.2260761+00$ \\
$-.1108476+00$
\end{tabular} & $\begin{array}{l}.8828317+01 \\
.1004028+01 \\
.2209051+00 \\
.7031486-01 \\
.282457 i-01\end{array}$ & $\begin{array}{l}.1352779+03 \\
.5477869+02 \\
.3122047+02 \\
.2105902+02 \\
.1563714+02\end{array}$ & \begin{tabular}{|l|}
$-.8837434+02$ \\
$-.2035962+02$ \\
$-.7644583+01$ \\
$-.3737462+01$ \\
$-.2148052+01$
\end{tabular} & $\begin{array}{l}.7130301+02 \\
.1060411+02 \\
.2865666+01 \\
.1077223+01 \\
.4974850+00\end{array}$ & \begin{tabular}{|l|}
$-.7112935+02$ \\
$-.7124064+01$ \\
$-.1434309+01$ \\
$-.4271379+00$ \\
$-.1628252+00$
\end{tabular} & $\begin{array}{l}-.6191167+03 \\
-.2058364+03 \\
-.1031736+03 \\
-.6373810+02 \\
-.4444770+02\end{array}$ & $\begin{array}{l}.5804663+03 \\
.1155539+03 \\
.3914991+02 \\
.1770730+02 \\
.9566481+01\end{array}$ & \begin{tabular}{|}
$-.5788742+03$ \\
$-.7602279+02$ \\
$-.1884592+02$ \\
$-.6642625+01$ \\
$-.2916919+01$
\end{tabular} & $\begin{array}{l}.6774773+03 \\
.6063949+02 \\
.1129137+02 \\
.3171862+01 \\
.1154970+01\end{array}$ \\
\hline $\begin{array}{l}.750 \\
.775 \\
.800 \\
.825 \\
.850\end{array}$ & $\begin{array}{l}-.9106524+01 \\
-.8666709+01 \\
-.8264145+01 \\
-.7894325+01 \\
-.7553437+01\end{array}$ & $\begin{array}{l}.6042573+00 \\
.5514550+00 \\
.5054719+00 \\
.4651899+00 \\
.4297086+00\end{array}$ & $\begin{array}{l}-.8165069-01 \\
-.7079891-01 \\
-.6176610-01 \\
-.5419135-01 \\
-.4779541-01\end{array}$ & $\begin{array}{l}.1906125-01 \\
.1585957-01 \\
.1329683-01 \\
.1122733-01 \\
.9542392-02\end{array}$ & $\begin{array}{l}.1380438+02 \\
.1303156+02 \\
.1233613+02 \\
.1170740+02 \\
.1113652+02\end{array}$ & \begin{tabular}{|}
$-.1699538+01$ \\
$-.1524744+01$ \\
$-.1374918+01$ \\
$-.1245653+01$ \\
$-.1133450+01$
\end{tabular} & $\begin{array}{l}.3572215+00 \\
.3060960+00 \\
.2640395+00 \\
.2291677+00 \\
.2000394+00\end{array}$ & \begin{tabular}{|}
$-.1074525+00$ \\
$-.8847997-01$ \\
$-.7345038-01$ \\
$-.6143328-01$ \\
$-.5174168-01$
\end{tabular} & $\begin{array}{l}-.3827307+02 \\
-.3572874+02 \\
-.3347101+02 \\
-.3145674+02 \\
-.2965070+02\end{array}$ & $\begin{array}{l}.7370382+01 \\
.6530819+01 \\
.5819563+01 \\
.5212764+01 \\
.4691705+01\end{array}$ & $\begin{array}{c}-.2050108+01 \\
-.1739312+01 \\
-.1486170+01 \\
-.1278253+01 \\
-.1106150+01\end{array}$ & $\begin{array}{l}.7474701+00 \\
.6099395+00 \\
.5019725+00 \\
.4163896+00 \\
.3479376+00\end{array}$ \\
\hline $\begin{array}{l}.875 \\
.900 \\
.925 \\
.950 \\
.975\end{array}$ & $\begin{array}{l}-.7238237+01 \\
-.6945946+01 \\
-.6674169+01 \\
-.6420834+01 \\
-.6184137+01\end{array}$ & $\begin{array}{l}.3982969+00 \\
.3703563+00 \\
.3453938+00 \\
.3230000+00 \\
.3028332+00\end{array}$ & $\begin{array}{l}-.4236016-01 \\
-.3771366-01 \\
-.3371925-01 \\
-.3026749-01 \\
-.2727002-01\end{array}$ & $\begin{array}{l}.8160038-02 \\
.7017830-02 \\
.6067750-02 \\
.5272539-02 \\
.4603047-02\end{array}$ & $\begin{array}{l}.1061608+02 \\
.1013989+02 \\
.9702672+01 \\
.9299966+01 \\
.8927939+01\end{array}$ & \begin{tabular}{|}
$-.1035509+01$ \\
$-.9495703+00$ \\
$-.8737942+00$ \\
$-.8066742+00$ \\
$-.7469674+00$
\end{tabular} & $\begin{array}{l}.1755415+00 \\
.1548062+00 \\
.1371507+00 \\
.1220335+00 \\
.1090218+00\end{array}$ & \begin{tabular}{|}
$-.4386263-01$ \\
$-.3740904-01$ \\
$-.3208590-01$ \\
$-.2766636-01$ \\
$-.2397440-01$
\end{tabular} & $\begin{array}{l}-.2802390+02 \\
-.2655229+02 \\
-.2521577+02 \\
-.2399746+02 \\
-.2288307+02\end{array}$ & $\begin{array}{l}.4241563+01 \\
.3850497+01 \\
.3508969+01 \\
.3209239+01 \\
.2944984+01\end{array}$ & $\begin{array}{l}-.9626580+00 \\
-.8422134+00 \\
-.7404765+00 \\
-.6540348+00 \\
-.5801825+00\end{array}$ & $\begin{array}{l}.2927274+00 \\
.2478486+00 \\
.2111010+00 \\
.1808051+00 \\
.1556676+00\end{array}$ \\
\hline $\begin{array}{l}1.000 \\
1.020 \\
1.040 \\
1.060 \\
1.080\end{array}$ & $\begin{array}{l}-.5962500+01 \\
-.5795098+01 \\
-.5635814+01 \\
-.5484075+01 \\
-.5339360+01\end{array}$ & $\begin{array}{l}.2846063+00 \\
.2712573+00 \\
.2588869+00 \\
.2474010+00 \\
.2367164+00\end{array}$ & $\begin{array}{l}-.2465508-01 \\
-.2279874-01 \\
-.2112430-01 \\
-.1961014-01 \\
-.1823766-01\end{array}$ & $\begin{array}{l}.4036291-02 \\
.3644458-02 \\
.3298998-02 \\
.2993507-02 \\
.2722584-02\end{array}$ & $\begin{array}{l}.8583300+01 \\
.8325379+01 \\
.8081874+01 \\
.7851634+01 \\
.7633628+01\end{array}$ & \begin{tabular}{|}
$-.6936423+00$ \\
$-.6549932+00$ \\
$-.6194981+00$ \\
$-.5868289+00$ \\
$-.5566980+00$
\end{tabular} & $\begin{array}{l}.9776733-01 \\
.8983730-01 \\
.8273007-01 \\
.7634321-01 \\
.7058903-01\end{array}$ & \begin{tabular}{|}
$-.2087236-01$ \\
$-.1874178-01$ \\
$-.1687391-01$ \\
$-.1523118-01$ \\
$-.1378209-01$
\end{tabular} & $\begin{array}{l}-.2186046+02 \\
-.2110142+02 \\
-.2038981+02 \\
-.1972151+02 \\
-.1909284+02\end{array}$ & $\begin{array}{l}.2711000+01 \\
.2542688+01 \\
.2389113+01 \\
.2248657+01 \\
.2119912+01\end{array}$ & $\begin{array}{r}-.5167584+00 \\
-.4723474+00 \\
-.4327570+00 \\
-.3973644+00 \\
-.3656390+00\end{array}$ & $\begin{array}{l}.1346841+00 \\
.1203541+00 \\
.1078523+00 \\
.9690966-01 \\
.8730156-01\end{array}$ \\
\hline $\begin{array}{l}1.100 \\
1.120 \\
1.140 \\
1.160 \\
1.180\end{array}$ & $\begin{array}{l}-.5201197+01 \\
-.5069153+01 \\
-.4942833+01 \\
-.4821874+01 \\
-.4705946+01\end{array}$ & $\begin{array}{l}.2267594+00 \\
.2174648+00 \\
.2087743+00 \\
.2006359+00 \\
.1930031+00\end{array}$ & $\begin{array}{l}-.1699075-01 \\
-.1585543-01 \\
-.1481955-01 \\
-.1387250-01 \\
-.1300499-01\end{array}$ & $\begin{array}{l}.2481658-02 \\
.2266841-02 \\
.2074822-02 \\
.1902763-02 \\
.1748233-02\end{array}$ & $\begin{array}{l}.7426925+01 \\
.7230685+01 \\
.7044150+01 \\
.6866628+01 \\
.6697495+01\end{array}$ & $\begin{array}{l}-.5288532+00 \\
-.5030723+00 \\
-.4791588+00 \\
-.4569390+00 \\
-.4362583+00\end{array}$ & $\begin{array}{l}.6539216-01 \\
.6068764-01 \\
.5641927-01 \\
.5253828-01 \\
.4900223-01\end{array}$ & $\begin{array}{l}-.1250013-01 \\
-.1136288-01 \\
-.1035133-01 \\
-.9449285-02 \\
-.8642939-02\end{array}$ & $\begin{array}{l}-.1850054+02 \\
-.1794165+02 \\
-.1741354+02 \\
-.1691384+02 \\
-.1644040+02\end{array}$ & $\begin{array}{l}.2001650+01 \\
.1892796+01 \\
.1792405+01 \\
.1699646+01 \\
.1613785+01\end{array}$ & $\begin{array}{l}-.3371275+00 \\
-.3114410+00 \\
-.2882448+00 \\
-.2672500+00 \\
-.2482063+00\end{array}$ & $\begin{array}{l}.7883983-01 \\
.7136620-01 \\
.6474703-01 \\
.5886910-01 \\
.5363610-01\end{array}$ \\
\hline $\begin{array}{l}1.200 \\
1.220 \\
1.240 \\
1.260 \\
1.280\end{array}$ & $\begin{array}{l}-.4594743+01 \\
-.4487984+01 \\
-.4385409+01 \\
-.4286779+01 \\
-.4191873+01\end{array}$ & $\begin{array}{l}.1858343+00 \\
.1790919+00 \\
.1727423+00 \\
.1667549+00 \\
.1611021+00\end{array}$ & \begin{tabular}{|}
$-.1220887-01$ \\
$-.1147695-01$ \\
$-.1080290-01$ \\
$-.1018114-01$ \\
$-.9606678-02$
\end{tabular} & $\begin{array}{l}.1609135-02 \\
.1483658-02 \\
.1370235-02 \\
.1267503-02 \\
.1174277-02\end{array}$ & $\begin{array}{l}.6536179+01 \\
.6382159+01 \\
.6234960+01 \\
.6094145+01 \\
.5959314+01\end{array}$ & $\begin{array}{l}-.4169796+00 \\
-.3989800+00 \\
-.3821499+00 \\
-.3663909+00 \\
-.3516148+00\end{array}$ & $\begin{array}{l}.4577407-01 \\
.4282138-01 \\
.4011571-01 \\
.3763202-01 \\
.3534823-01\end{array}$ & \begin{tabular}{|}
$-.7920441-02$ \\
$-.7271613-02$ \\
$-.6687678-02$ \\
$-.6161045-02$ \\
$-.5685136-02$
\end{tabular} & $\begin{array}{l}-.1599127+02 \\
-.1556470+02 \\
-.1515909+02 \\
-.1477298+02 \\
-.1440505+02\end{array}$ & $\begin{array}{l}.1534173+01 \\
.1460232+01 \\
.1391449+01 \\
.1327367+01 \\
.1267577+01\end{array}$ & $\begin{array}{l}-.2308962+00 \\
-.2151304+00 \\
-.2007434+00 \\
-.1875901+00 \\
-.1755433+00\end{array}$ & $\begin{array}{l}.4896588-01 \\
.4478812-01 \\
.4104243-01 \\
.3767682-01 \\
.3464638-01\end{array}$ \\
\hline $\begin{array}{l}1.300 \\
1.320 \\
1.340 \\
1.360 \\
1.380\end{array}$ & $\begin{array}{l}-.4100485+01 \\
-.4012424+01 \\
-.3927514+01 \\
-.3845590+01 \\
-.3766497+01\end{array}$ & $\begin{array}{l}.1557590+00 \\
.1507028+00 \\
.1459130+00 \\
.1413707+00 \\
.1370587+00\end{array}$ & \begin{tabular}{|}
$-.9075118-02$ \\
$-.8582525-02$ \\
$-.8125394-02$ \\
$-.7700587-02$ \\
$-.7305295-02$
\end{tabular} & $\begin{array}{l}.1089520-02 \\
.1012326-02 \\
.9418981-03 \\
.8775369-03 \\
.8186250-03\end{array}$ & $\begin{array}{l}.5830098+01 \\
.5706160+01 \\
.5587186+01 \\
.5472888+01 \\
.5363000+01\end{array}$ & $\begin{array}{l}-.3377420+00 \\
-.3247007+00 \\
-.3124262+00 \\
-.3008598+00 \\
-.2899481+00\end{array}$ & $\begin{array}{l}.3324481-01 \\
.3130446-01 \\
.2951182-01 \\
.2785321-01 \\
.2631644-01\end{array}$ & \begin{tabular}{|l|}
$-.5254229-02$ \\
$-.4863339-02$ \\
$-.4508109-02$ \\
$-.4184722-02$ \\
$-.3889827-02$
\end{tabular} & $\begin{array}{l}-.1405408+02 \\
-.1371897+02 \\
-.1339869+02 \\
-.1309232+02 \\
-.1279899+02\end{array}$ & $\begin{array}{l}.1211711+01 \\
.1159442+01 \\
.1110474+01 \\
.1064539+01 \\
.1021398+01\end{array}$ & $\begin{array}{l}-.1644908+00 \\
-.1543338+00 \\
-.1449846+00 \\
-.1363658+00 \\
-.1284084+00\end{array}$ & $\begin{array}{l}.3191220-01 \\
.2944052-01 \\
.2720193-01 \\
.2517076-01 \\
.2332456-01\end{array}$ \\
\hline $\begin{array}{l}1.400 \\
1.420 \\
1.440 \\
1.460 \\
1.480\end{array}$ & $\begin{array}{l}-.3690094+01 \\
-.3616247+01 \\
-.3544829+01 \\
-.3475725+01 \\
-.3408825+01\end{array}$ & $\begin{array}{l}.1329614+00 \\
.1290643+00 \\
.1253544+00 \\
.1218194+00 \\
.1184482+00\end{array}$ & $\begin{array}{l}-.6936996-02 \\
-.6593418-02 \\
-.6272520-02 \\
-.5972455-02 \\
-.5691555-02\end{array}$ & $\begin{array}{l}.7646175-03 \\
.7150316-03 \\
.6694390-03 \\
.6274592-03 \\
.5887531-03\end{array}$ & $\begin{array}{l}.5257273+01 \\
.5155479+01 \\
.5057405+01 \\
.4962853+01 \\
.4871639+01\end{array}$ & $\begin{array}{l}-.2796430+00 \\
-.2699002+00 \\
-.2606797+00 \\
-.2519448+00 \\
-.2436620+00\end{array}$ & $\begin{array}{l}.2489059-01 \\
.2356591-01 \\
.2233365-01 \\
.2118594-01 \\
.2011568-01\end{array}$ & $\begin{array}{l}-.3620475-02 \\
-.3374066-02 \\
-.3148301-02 \\
-.2941146-02 \\
-.2750794-02\end{array}$ & $\begin{array}{l}-.1251791+02 \\
-.1224836+02 \\
-.1198965+02 \\
-.1174117+02 \\
-.1150233+02\end{array}$ & $\begin{array}{l}.9808317+00 \\
.9426436+00 \\
.9066544+00 \\
.8727012+00 \\
.8406358+00\end{array}$ & $\begin{array}{l}-.1210511+00 \\
-.1142391+00 \\
-.1079234+00 \\
-.1020602+00 \\
-.9661029-01\end{array}$ & $\begin{array}{l}.2164364-01 \\
.2011068-01 \\
.1871044-01 \\
.1742947-01 \\
.1625585-01\end{array}$ \\
\hline $\begin{array}{l}1.500 \\
1.540 \\
1.580 \\
1.620 \\
1.660\end{array}$ & $\begin{array}{l}-.3344026+01 \\
-.3220351+01 \\
-.3103994+01 \\
-.2994332+01 \\
-.2890808+01\end{array}$ & $\begin{array}{l}.1152306+00 \\
.1092191+00 \\
.1037173+00 \\
.9866765-01 \\
.9402029-01\end{array}$ & \begin{tabular}{|}
$-.5428309-02$ \\
$-.4949424-02$ \\
$-.4526267-02$ \\
$-.4150927-02$ \\
$-.3816804-02$
\end{tabular} & $\begin{array}{l}.5530182-03 \\
.4894089-03 \\
.4347880-03 \\
.3876680-03 \\
.3468411-03\end{array}$ & $\begin{array}{l}.4783590+01 \\
.4616359+01 \\
.4459999+01 \\
.4313493+01 \\
.4175946+01\end{array}$ & $\begin{array}{c}-.2358005+00 \\
-.2212316+00 \\
-.2080389+00 \\
-.1960539+00 \\
-.1851327+00\end{array}$ & $\begin{array}{l}.1911649-01 \\
.1730879-01 \\
.1572291-01 \\
.1432595-01 \\
.1309071-01\end{array}$ & $\begin{array}{l}-.2575640-02 \\
-.2265358-02 \\
-.2000599-02 \\
-.1773586-02 \\
-.1578045-02\end{array}$ & $\begin{array}{l}-.1127260+02 \\
-.1083852+02 \\
-.1043532+02 \\
-.1005991+02 \\
-.9709564+01\end{array}$ & $\begin{array}{l}.8103229+00 \\
.7544709+00 \\
.7042768+00 \\
.6590101+00 \\
.6180529+00\end{array}$ & $\begin{array}{c}-.9153826-01 \\
-.8240377-01 \\
-.7443770-01 \\
-.6746083-01 \\
-.6132563-01\end{array}$ & $\begin{array}{l}.1517905-01 \\
.1327944-01 \\
.1166733-01 \\
.1029231-01 \\
.9113914-02\end{array}$ \\
\hline $\begin{array}{l}1.700 \\
1.740 \\
1.780 \\
1.820 \\
1.860\end{array}$ & $\begin{array}{l}-.2792925+01 \\
-.2700241+01 \\
-.2612356+01 \\
-.2528910+01 \\
-.2449581+01\end{array}$ & $\begin{array}{l}.8973227-01 \\
.8576623-01 \\
.8208963-01 \\
.7867397-01 \\
.7549423-01\end{array}$ & $\left|\begin{array}{l}-.3518361-02 \\
-.3250930-02 \\
-.3010560-02 \\
-.2793888-02 \\
-.2598041-02\end{array}\right|$ & $\begin{array}{l}.3113207-03 \\
.2802961-03 \\
.2530977-03 \\
.2291698-03 \\
.2080486-03\end{array}$ & $\begin{array}{l}.4046565+01 \\
.3924651+01 \\
.3809579+01 \\
.3700793+01 \\
.3597794+01\end{array}$ & $\left|\begin{array}{c}-.1751523+00 \\
-.1660069+00 \\
-.1576051+00 \\
-.1498676+00 \\
-.1427254+00\end{array}\right|$ & $\begin{array}{l}.1199449-01 \\
.1101831-01 \\
.1014621-01 \\
.9364685-02 \\
.8662282-02\end{array}$ & $\left|\begin{array}{|}-.1408884-02 \\
-.1261943-02 \\
-.1133807-02 \\
-.1021659-02 \\
-.9231578-03\end{array}\right|$ & $\begin{array}{l}-.9381905+01 \\
-.9074843+01 \\
-.8786530+01 \\
-.8515330+01 \\
-.8259790+01\end{array}$ & $\begin{array}{l}.5808802+00 \\
.5470433+00 \\
.5161575+00 \\
.4878915+00 \\
.4619586+00\end{array}$ & \begin{tabular}{|}
$-.5591000-01$ \\
$-.5111227-01$ \\
$-.4684744-01$ \\
$-.4304401-01$ \\
$-.3964164-01$
\end{tabular} & $\begin{array}{l}.8099470-02 \\
.7222444-02 \\
.6461157-02 \\
.5797800-02 \\
.5217671-02\end{array}$ \\
\hline
\end{tabular}


$(m=6)$

\begin{tabular}{|c|c|c|c|c|c|c|c|c|c|c|c|c|}
\hline$T^{*}$ & $\mathrm{~B}^{*(0)}$ & $\mathrm{B}^{*(1)}$ & $\mathrm{B}^{*(2)}$ & $\mathrm{B}^{*(3)}$ & $\mathrm{T}^{*} \frac{\partial \mathrm{B}^{*(0)}}{\partial \mathrm{T}^{*}}$ & $\mathrm{~T}^{*} \frac{\partial \mathrm{B}^{*(1)}}{\partial \mathrm{T}^{*}}$ & $\frac{\partial B^{*(2)}}{\partial T^{*}}$ & $\mathrm{~T}^{*} \frac{\partial \mathrm{B}^{*(3)}}{\partial \mathrm{T}^{*}}$ & $\mathrm{~T}^{* 2} \frac{\partial^{2} \mathrm{~B}^{*(0)}}{\partial \mathrm{T}^{* 2}}$ & $\mathrm{~T}^{* 2} \frac{\partial^{2} \mathrm{~B}^{*(1)}}{\partial \mathrm{T}^{* 2}}$ & $\mathrm{~T}^{* 2} \frac{\partial^{2} \mathrm{~B}^{*(2)}}{\partial \mathrm{T}^{* 2}}$ & $\mathrm{~T}^{* 2} \frac{\partial^{2} \mathrm{~B}^{*(3)}}{\partial \mathrm{T}^{* 2}}$ \\
\hline $\begin{array}{l}1.900 \\
1.940 \\
1.980 \\
2.020 \\
2.060\end{array}$ & $\begin{array}{l}-.2374074+01 \\
-.2302122+01 \\
-.2233485+01 \\
-.2167940+01 \\
-.2105287+01\end{array}$ & $\begin{array}{l}.7252835-01 \\
.6975682-01 \\
.6716236-01 \\
.6472958-01 \\
.6244477-01\end{array}$ & $\begin{array}{l}-.2420556-02 \\
-.2259312-02 \\
-.2112476-02 \\
-.1978457-02 \\
-.1855874-02\end{array}$ & $\begin{array}{l}.1893456-03 \\
.1727337-03 \\
.1579364-03 \\
.1447192-03 \\
.1328821-03\end{array}$ & $\begin{array}{l}.3500135+01 \\
.3407415+01 \\
.3319268+01 \\
.3235368+01 \\
.3155416+01\end{array}$ & $\begin{array}{l}-.1361184+00 \\
-.1299935+00 \\
-.1243045+00 \\
-.1190102+00 \\
-.1140744+00\end{array}$ & $\begin{array}{l}.8029235-02 \\
.7457184-02 \\
.6938948-02 \\
.6468333-02 \\
.6039982-02\end{array}$ & $\begin{array}{l}-.8363557-03 \\
-.7596199-03 \\
-.6915776-03 \\
-.6310696-03 \\
-.5771131-03\end{array}$ & $\begin{array}{l}-.8018614+01 \\
-.7790643+01 \\
-.7574839+01 \\
-.7370267+01 \\
-.7176084+01\end{array}$ & $\begin{array}{l}.4381099+00 \\
.4161286+00 \\
.3958250+00 \\
.3770329+00 \\
.3596058+00\end{array}$ & $\begin{array}{l}-.3658912-01 \\
-.3384287-01 \\
-.3136559-01 \\
-.2912529-01 \\
-.2709440-01\end{array}$ & $\begin{array}{l}.4708568-02 \\
.4260322-02 \\
.3864415-02 \\
.3513687-02 \\
.3202091-02\end{array}$ \\
\hline $\begin{array}{l}2.100 \\
2.140 \\
2.180 \\
2.220 \\
2.260\end{array}$ & $\begin{array}{l}-.2045341+01 \\
-.1987932+01 \\
-.1932906+01 \\
-.1880120+01 \\
-.1829440+01\end{array}$ & $\begin{array}{l}.6029564-01 \\
.5827121-01 \\
.5636157-01 \\
.5455781-01 \\
.5285190-01\end{array}$ & $\begin{array}{l}-.1743519-02 \\
-.1640335-02 \\
-.1545395-02 \\
-.1457882-02 \\
-.1377074-02\end{array}$ & $\begin{array}{l}.1222544-03 \\
.1126894-03 \\
.1040610-03 \\
.9626009-04 \\
.8919243-04\end{array}$ & $\begin{array}{l}.3079140+01 \\
.3006294+01 \\
.2936652+01 \\
.2870009+01 \\
.2806176+01\end{array}$ & $\begin{array}{l}-.1094648+00 \\
-.1051530+00 \\
-.1011132+00 \\
-.9732256-01 \\
-.9376065-01\end{array}$ & $\begin{array}{l}.5649242-02 \\
.5292061-02 \\
.4964900-02 \\
.4664658-02 \\
.4388609-02\end{array}$ & $\begin{array}{l}-.5288717-03 \\
-.4856311-03 \\
-.4467791-03 \\
-.4117892-03 \\
-.3802074-03\end{array}$ & $\begin{array}{l}-.6991530+01 \\
-.6815915+01 \\
-.6648614+01 \\
-.6489056+01 \\
-.6336723+01\end{array}$ & $\begin{array}{l}.3434147+00 \\
.3283450+00 \\
.3142953+00 \\
.3011752+00 \\
.2889041+00\end{array}$ & $\begin{array}{l}-.2524910-01 \\
-.2356872-01 \\
-.2203529-01 \\
-.2063312-01 \\
-.1934848-01\end{array}$ & $\begin{array}{l}.2924502-02 \\
.2676560-02 \\
.2454541-02 \\
.2255256-02 \\
.2075964-02\end{array}$ \\
\hline $\begin{array}{l}2.300 \\
2.340 \\
2.380 \\
2.420 \\
2.460\end{array}$ & $\begin{array}{l}-.1780747+01 \\
-.1733927+01 \\
-.1688876+01 \\
-.1645497+01 \\
-.1603701+01\end{array}$ & & & & & & & $\begin{array}{l}-.3516407-03 \\
-.3257482-03 \\
-.3022331-03 \\
-.2808366-03 \\
-.2613322-03\end{array}$ & $\begin{array}{l}-.6191141+01 \\
-.6051874+01 \\
-.5918526+01 \\
-.5790731+01 \\
-.5668152+01\end{array}$ & & $\begin{array}{l}-.1816934-01 \\
-.1708507-01 \\
-.1608634-01 \\
-.1516487-01 \\
-.1431333-01\end{array}$ & $\begin{array}{l}.1914300-02 \\
.1768221-02 \\
.1635954-02 \\
.1515956-02 \\
.1406882-02\end{array}$ \\
\hline $\begin{array}{l}2.500 \\
2.600 \\
2.700 \\
2.800 \\
2.900\end{array}$ & $\begin{array}{l}-.1301902+01 \\
-.1228075+01\end{array}$ & & $1001843-02$ & & & & $\begin{array}{l}.3123637-02 \\
.2742779-02 \\
.2422803-02 \\
.2151922-02 \\
.1920992-02\end{array}$ & $\begin{array}{l}-.2435210-03 \\
-.2053053-03 \\
-.1744211-03 \\
-.1492313-03 \\
-.1285114-03\end{array}$ & $\begin{array}{l}-.5550479+01 \\
-.5275938+01 \\
-.5026414+01 \\
-.4798660+01 \\
-.4589966+01\end{array}$ & $\begin{array}{l}.2295449+00 \\
.2103930+00 \\
.1936960+00 \\
.1790468+00 \\
.1661190+00\end{array}$ & $\begin{array}{l}-.1352520-01 \\
-.1179575-01 \\
-.1035298-01 \\
-.9139728-02 \\
-.8111962-02\end{array}$ & $\begin{array}{l}.1307556-02 \\
.1095428-02 \\
.9251034-03 \\
.7870350-03 \\
.6741272-03\end{array}$ \\
\hline $\begin{array}{l}3.000 \\
3.100 \\
3.200 \\
3.300 \\
3.400\end{array}$ & $\begin{array}{l}-.1037175+01 \\
-.9820703+00 \\
-.9305557+00\end{array}$ & $\begin{array}{l}.3092412-01 \\
.2937495-01 \\
.2795810-01 \\
.2665808-01\end{array}$ & $\begin{array}{l}-.4318067-03 \\
-.3955025-03\end{array}$ & & $.1694340+01$ & $\begin{array}{l}-.5345700-01 \\
-.5025570-01 \\
-.4736691-01 \\
-.4474985-01 \\
-.4237027-01\end{array}$ & $\begin{array}{l}.1722845-02 \\
.1551807-02 \\
.1403348-02 \\
.1273819-02 \\
.1160261-02\end{array}$ & $\begin{array}{l}-.1113344-03 \\
-.9699097-04 \\
-.8493295-04 \\
-.7473253-04 \\
-.6605298-04\end{array}$ & $\begin{array}{l}-.4398048+01 \\
-.4220973+01 \\
-.4057093+01 \\
-.3904992+01 \\
-.3763449+01\end{array}$ & $\begin{array}{l}.1546491+00 \\
.1444223+00 \\
.1352619+00 \\
.1270218+00 \\
.1195801+00\end{array}$ & $\begin{array}{l}-.7235410-02 \\
-.6483125-02 \\
-.5833725-02 \\
-.5270100-02 \\
-.4778449-02\end{array}$ & $\begin{array}{l}.5037266-03 \\
.4390558-03 \\
.3846114-03 \\
.3384982-03\end{array}$ \\
\hline $\begin{array}{l}3.500 \\
3.600 \\
3.700 \\
3.800 \\
3.900\end{array}$ & $\begin{array}{l}-.8822999+00 \\
-.8370113+00 \\
-.7944319+00 \\
-.7543325+00 \\
-.7165088+00\end{array}$ & $\begin{array}{l}.2546166-01 \\
.2435747-01 \\
.2333573-01 \\
.2238792-01 \\
.2150666-01\end{array}$ & $\begin{array}{l}-.2863279-03 \\
-.2657663-03\end{array}$ & & & $\begin{array}{l}-.4019923-01 \\
-.3821212-01 \\
-.3638793-01 \\
-.3470860-01 \\
-.3315855-01\end{array}$ & & $\begin{array}{l}-.5862710-04 \\
-.5224121-04 \\
-.4672320-04 \\
-.4193353-04 \\
-.3775831-04\end{array}$ & & & $\begin{array}{l}-.4347555-02 \\
-.3968241-02 \\
-.3632949-02 \\
-.3335413-02 \\
-.3070414-02\end{array}$ & $\begin{array}{l}.2992199-03 \\
.2655858-03 \\
.2366413-03 \\
.2116158-03 \\
.1898830-03\end{array}$ \\
\hline $\begin{array}{l}4.000 \\
4.100 \\
4.200 \\
4.300 \\
4.400\end{array}$ & $\begin{array}{l}-.6807783+00 \\
-.6469775+00 \\
-.6149590+00 \\
-.5845903+00 \\
-.5557513+00\end{array}$ & $\begin{array}{l}.2068547-01 \\
.1991867-01 \\
.1920126-01 \\
.1852883-01 \\
.1789744-01\end{array}$ & $\begin{array}{l}-.2304617-03 \\
-.2152582-03 \\
-.2014345-03 \\
-.1888337-03\end{array}$ & & & & $\begin{array}{l}.7045662-03 \\
.6540211-03 \\
.6083918-03 \\
.5670847-03 \\
.5295910-03\end{array}$ & $\begin{array}{l}-.3410405-04 \\
-.3089359-04 \\
-.2806287-04 \\
-.2555843-04 \\
-.2333550-04\end{array}$ & & & $\begin{array}{l}-.2833578-02 \\
-.2621219-02 \\
-.2430219-02 \\
-.2257927-02 \\
-.2102079-02\end{array}$ & $\begin{array}{l}.1709313-03 \\
.1543396-03 \\
.1397599-03 \\
.1269031-03 \\
.1155277-03\end{array}$ \\
\hline $\begin{array}{l}4.500 \\
4.600 \\
4.700 \\
4.800 \\
4.900\end{array}$ & $\begin{array}{l}-.5283334+00 \\
-.5022379+00 \\
-.4773749+00 \\
-.4536624+00 \\
-.4310254+00\end{array}$ & $\begin{array}{l}.1730361-01 \\
.1674421-01 \\
.1621646-01 \\
.1571786-01 \\
.1524615-01\end{array}$ & $\begin{array}{l}-.1773202-03 \\
-.1667761-03 \\
-.1570991-03 \\
-.1481994-03 \\
-.1399984-03\end{array}$ & & & $\begin{array}{l}-.2592564-01 \\
-.2498409-01 \\
-.2410060-01 \\
-.2327028-01 \\
-.2248871-01\end{array}$ & $\begin{array}{l}.4954726-03 \\
.4643510-03 \\
.4358982-03 \\
.4098284-03 \\
.3858925-03\end{array}$ & $\begin{array}{l}-.2135635-04 \\
-.1958909-04 \\
-.1800662-04 \\
-.1658585-04 \\
-.1530702-04\end{array}$ & & & $\begin{array}{l}-.1960733-02 \\
-.1832220-02 \\
-.1715093-02 \\
-.1608103-02 \\
-.1510159-02\end{array}$ & $\begin{array}{l}.1054308-03 \\
.9644166-04 \\
.8841569-04 \\
.8122998-04 \\
.7477969-04\end{array}$ \\
\hline $\begin{array}{l}5.000 \\
5.100 \\
5.200 \\
5.300 \\
5.400\end{array}$ & $\begin{array}{l}-.4093955+00 \\
-.3887095+00 \\
-.3689097+00 \\
-.3499427+00 \\
-.3317593+00\end{array}$ & $\begin{array}{l}.1437549-01 \\
.1397304-01 \\
.1359043-01 \\
.1322630-01\end{array}$ & $\begin{array}{l}-.1254244-03 \\
-.1189366-03 \\
-.1129159-03 \\
-.1073197-03\end{array}$ & $\begin{array}{l}.3521265-05 \\
.3276003-05 \\
.3052591-05 \\
.2848682-05\end{array}$ & $\begin{array}{l}.9840679+00 \\
.9615750+00\end{array}$ & $\begin{array}{l}-.2175197-01 \\
-.2105649-01 \\
-.2039911-01 \\
-.1977692-01 \\
-.1918733-01\end{array}$ & $\begin{array}{l}.3638719-03 \\
.3435751-03 \\
.3248329-03 \\
.3074961-03 \\
.2914326-03\end{array}$ & $\begin{array}{l}-.1415316-04 \\
-.1310962-04 \\
-.1216378-04 \\
-.1130465-04 \\
-.1052269-04\end{array}$ & $\begin{array}{l}-.2358925+01 \\
-.2303787+01 \\
-.2251012+01 \\
-.2200451+01 \\
-.2151967+01\end{array}$ & $\begin{array}{l}.5752953-01 \\
.5552873-01 \\
.5364428-01 \\
.5186697-01 \\
.5018847-01\end{array}$ & $\begin{array}{l}-.1420310-02 \\
-.1337724-02 \\
-.1261671-02 \\
-.1191505-02 \\
-.1126659-02\end{array}$ & $\begin{array}{l}.6373887-04 \\
.5900470-04 \\
.5471497-04 \\
.5081978-04\end{array}$ \\
\hline $\begin{array}{l}5.500 \\
5.600 \\
5.800 \\
6.000 \\
6.200\end{array}$ & $\begin{array}{l}-.3143142+00 \\
-.2975654+00 \\
-.2660038+00 \\
-.2367954+00 \\
-.2096988+00\end{array}$ & $\begin{array}{l}.1254856-01 \\
.1193100-01 \\
.1136624-01 \\
.1084802-01\end{array}$ & $\begin{array}{l}-.8848111-04 \\
-.8079536-04 \\
-.7402846-04\end{array}$ & $\begin{array}{l}.2491408-05 \\
.2190563-05 \\
.1935557-05 \\
.1718080-05\end{array}$ & $\begin{array}{l}.9399618+00 \\
.9191779+00 \\
.8799149+00 \\
.8434520+00 \\
.8095012+00\end{array}$ & $\begin{array}{l}-.1862798-01 \\
-.1809671-01 \\
-.1711079-01 \\
-.1621587-01 \\
-.1540048-01\end{array}$ & $\begin{array}{l}.2765249-03 \\
.2626687-03 \\
.2377474-03 \\
.2160343-03 \\
.1970181-03\end{array}$ & $\begin{array}{l}-.9809570-05 \\
-.9158011-05 \\
-.8014773-05 \\
-.7050463-05 \\
-.6231898-05\end{array}$ & $\begin{array}{l}-.2105436+01 \\
-.2060742+01 \\
-.1976446+01 \\
-.1898320+01 \\
-.1825711+01\end{array}$ & $\begin{array}{l}.4860128-01 \\
.4709861-01 \\
.4432275-01 \\
.4181797-01 \\
.3954858-01\end{array}$ & $\begin{array}{l}-.1066629-02 \\
-.1010968-02 \\
-.9112069-03 \\
-.8246798-03 \\
-.7492251-03\end{array}$ & $\begin{array}{l}.4727568-04 \\
.4404476-04 \\
.3839386-04 \\
.3364719-04 \\
.2963388-04\end{array}$ \\
\hline
\end{tabular}


$(m=6)$

\begin{tabular}{|c|c|c|c|c|c|c|c|c|c|c|c|c|}
\hline $\mathrm{T}^{*}$ & $\mathrm{~B}^{*(0)}$ & $\mathrm{B}^{*(1)}$ & $\mathrm{B}^{*(2)}$ & $\mathrm{B}^{*(3)}$ & $\mathrm{T}^{*} \frac{\partial \mathrm{B}^{*(0)}}{\partial \mathrm{T}^{*}}$ & $\mathrm{~T}^{*} \frac{\partial \mathrm{B}^{*(1)}}{\partial \mathrm{T}^{*}}$ & $\mathrm{~T}^{*} \frac{\partial \mathrm{B}^{*(2)}}{\partial \mathrm{T}^{*}}$ & $\mathrm{~T}^{*} \frac{\partial \mathrm{B}^{*(3)}}{\partial \mathrm{T}^{*}}$ & $T^{* 2} \frac{\partial^{2} B^{*(0)}}{\partial T^{* 2}}$ & $\mathrm{~T}^{* 2} \frac{\partial^{2} \mathrm{~B} *(1)}{\partial \mathrm{T}^{* 2}}$ & $\mathrm{~T}^{* 2} \frac{\partial^{2} \mathrm{~B}^{*(2)}}{\partial \mathrm{T}^{* 2}}$ & $\mathrm{~T}^{* 2} \frac{\partial^{2} \mathrm{~B}^{*(3)}}{\partial \mathrm{T}^{* 2}}$ \\
\hline 6.400 & $.1845043+00$ & 1 & 4 & ( & 0 & 1 & 03 & 05 & $8055+01$ & 01 & 03 & 1799104 \\
\hline & & & & & $2+00$ & -01 & 48 & $-.4932799-05$ & $94861+01$ & & $248652-03$ & $.2329925-04$ \\
\hline & -.1 & $3078-02$ & $.5798512-04$ & 5 & $3777+00$ & $-.1334190-01$ & $23576-03$ & $-.4414860-05$ & $-.1635704+01$ & $.3387637-01$ & -.5733680 & $.2078734-04$ \\
\hline 000 & & $.9144814-02$ & $.5374066-04$ & $.1109983-05$ & $.6942731+00$ & $-.1276134-01$ & $.1406541-03$ & $-.3965698-05$ & $-.1580209+01$ & $.3229234-01$ & $-.5276382-03$ & $.1861623-04$ \\
\hline 7.200 & $-.9939856-01$ & $.8792942-02$ & $-.4992777-04$ & $.1003876-05$ & $.6697064+00$ & $-.1222424-01$ & $.1301852-03$ & $-.3574415-05$ & $-.1528046+01$ & $.3083334-01$ & $-.4868740-03$ & $.1673090-04$ \\
\hline 7.400 & $-.8136819-01$ & $.8464888-02$ & $.4649126-04$ & $.9107121-06$ & $64+00$ & $-.1172611-01$ & $.1207883-03$ & $-.3232099-05$ & $-.1478924+01$ & .2948590-01 & $-.4504051-03$ & $.1508656-04$ \\
\hline & & & & & & & & & & & & \\
\hline & 01 & & $0-04$ & 6 & +00 & -01 & 03 & $6312-05$ & $800+01$ & $8052-01$ & 381871 & $.1238037-04$ \\
\hline 8.000 & -.33 & & & & & & & & & $0359-01$ & & $.1126311-04$ \\
\hline 8.200 & $-.1922021-01$ & $.7349547-02$ & $.3567021-04$ & $6-06$ & -00 & $204-01$ & .91 & -.2 & -.130805 & $8-01$ & - & $.1027377-04$ \\
\hline 8.400 & $-.5798995-02$ & $.7111600-02$ & $.3353593-04$ & $.5827144-06$ & $.5481680+00$ & $.9698930-02$ & $.8573574-04$ & $-.2037950-05$ & $-.1270818+01$ & .2406 & $-.3155258-03$ & .939 \\
\hline 8.600 & & .68 & 4 & & & & & & & & & \\
\hline 8.800 & .1 & & 4 & & & & & & & & & \\
\hline 9.000 & & & 04 & & & & & -.1 & & & & \\
\hline 00 & 01 & $.6285853-02$ & $.2661118-04$ & $75-06$ & $.4856361+00$ & $-.8485061-02$ & $.6728617-04$ & $-.1470111-05$ & -.1138953 & $.2086413-01$ & -.2 & .67195 \\
\hline 9.400 & -01 & $.6106201-02$ & $.2520449-04$ & $.3941635-06$ & $.4717516+00$ & $.8223407-02$ & $.6356750-04$ & $-.1361574-05$ & $-.1109711+01$ & .2017999-01 & $-.2313714-03$ & .62111 \\
\hline & & & & & & & & & & & & \\
\hline 00 & 01 & & 04 & & & & & & & & & \\
\hline 10.000 & & .56 & 04 & & & -.7 & & -.1 & & 01 & & \\
\hline 10.200 & .879 & -02 & $231-04$ & .29 & $258+00$ & $-.7308013-02$ & $.5129722-04$ & $-.1019275-05$ & $-.1004869+01$ & $00-01$ & $-.1852815-03$ & .46157 \\
\hline 10.400 & .9 & .5332 & $.1956104-04$ & .2784 & $.4107265+00$ & -.7107338 & $.4876343-04$ & $-.9518475-06$ & $-.9813206+00$ & $.1728402-01$ & $-.1758155-03$ & .4 \\
\hline & & & & & & & & & & & & \\
\hline 10 & & & 04 & & -00 & -.6 & & -8 & & & & \\
\hline 11 & & & & & & & & & & & & \\
\hline & $.1314034+00$ & $67-02$ & $65-04$ & $0-06$ & $9+00$ & $-.6237209-02$ & .38 & -.65 & -8 & $60-01$ & -.1 & $.3095274-05$ \\
\hline 11.800 & $1435468+00$ & 02 & 4 & & & 2 & .35 & -.6 & -.8 & -01 & -.1 & .27 \\
\hline & & & & & & & & & & & & \\
\hline & & & 04 & & & & &.- & & & & -05 \\
\hline & & & 04 & & & & & & & & & \\
\hline & & -02 & $06-04$ & .1 & $6+00$ & -.49 & 04 & $56-06$ & $954+00$ & 64-01 & $-.8977231-04$ & $452-05$ \\
\hline 13.800 & $1915701+00$ & -02 & $56-05$ & .10 & .27 & -.47 & .23 & -.3 & -.6 & -01 & $-.8+2-x$ & .15 \\
\hline & & & & & & & & & & & & \\
\hline & & & & & & -.4 & & & & .1 & - . & -05 \\
\hline 15 & $1+0$ & $.3299396-02$ & 1205 & & & & & & & & & \\
\hline & & $.3036164-02$ & $-.0040034-05$ & $.6585596-07$ & $.2150082+00$ & $-.3902363-02$ & $.1632991-04$ & $-.2161164-06$ & $-.5699546+00$ & $.9194853-02$ & $-.5680152-04$ & $.9439779-06$ \\
\hline 17 & $2398747+0$ & & -05 & & & -.3 & & -.1 & -.5 & .8 & -04 & .76 \\
\hline & & & & & & & & & & & & \\
\hline & & & -05 & & & & & -.1 &  & -02 &.- & $.5252171-06$ \\
\hline & & & & & & & & & & & & \\
\hline & & $.2147298-02$ & | & $.2725110-07$ & $.1317041+00$ & $-.2712261-02$ & $.8400837-05$ & $-.8746456-07$ & $-.3938698+00$ & $.6294480-02$ & $-.2866049-04$ & $.3749459-06$ \\
\hline & & & & & & & & & & & -.2 & .320 \\
\hline & & & & & & & & & & & & \\
\hline & & & & & & & & & & $21-02$ & 04 & $.2402941-06$ \\
\hline & $.2932755+00$ & $.1724678-02$ & -.24 & & & & & & & & & \\
\hline & & .1 & $9-$ & 7 & .81 & -02 & $50-05$ & $-.4361525-07$ & -.2876847 & $.4712508-02$ & $7631-04$ & $.1844988-06$ \\
\hline & & & -.2 & .12 & & -.1 & -05 & $647-07$ & -.2713 & & & \\
\hline & & & .10 & & & & & -.34 & -.25 & & $139-04$ & .1 \\
\hline & & & $.1717605-05$ & .9790 & & -.17 & & & & & & .1 \\
\hline 30. & .3063 & .137 & -.15 & .88 & .54 & -.17 & & -.2 & -.2 & & $425-04$ & $.1155429-06$ \\
\hline & $.3095688+00$ & $.1268660-02$ & $-.1374568-05$ & $.7197794-08$ & $.4399182-01$ & $-.1569424-02$ & $.3100205-05$ & $-.2242037-07$ & $-.2055732+00$ & $.3577032-02$ & $-.1030725-04$ & $.9372164-07$ \\
\hline
\end{tabular}


$(m=6)$

\begin{tabular}{|c|c|c|c|c|c|c|c|c|c|c|c|c|}
\hline $\mathrm{T}^{*}$ & $\mathrm{~B}^{*(0)}$ & $\mathrm{B}^{*(1)}$ & B & $\mathrm{B}^{*(3)}$ & $\mathrm{T}^{*} \frac{\partial \mathrm{B}^{*(0)}}{\partial \mathrm{T}^{*}}$ & $\mathrm{~T}^{*} \frac{\partial \mathrm{B}^{*(1)}}{\partial \mathrm{T}^{*}}$ & $\mathrm{~T}^{*} \frac{\partial \mathrm{B}^{*(2)}}{\partial \mathrm{T}^{*}}$ & $\mathrm{~T}^{*} \frac{\partial \mathrm{B}^{*(3)}}{\partial \mathrm{T}^{*}}$ & $\mathrm{~T}^{* 2} \frac{\partial^{2} \mathrm{~B}^{*(0)}}{\partial \mathrm{T}^{* 2}}$ & $\mathrm{~T}^{* 2} \frac{\partial^{2} \mathrm{~B} *(1)}{\partial \mathrm{T}^{* 2}}$ & $\mathrm{~T}^{* 2} \frac{\partial^{2} \mathrm{~B}^{*(2)}}{\partial \mathrm{T}^{* 2}}$ & $T^{* 2} \frac{\partial^{2} B^{*(3)}}{\partial T^{* 2}}$ \\
\hline $\begin{array}{l}34.000 \\
36.000 \\
38.000 \\
40.000 \\
42.000\end{array}$ & $\begin{array}{l}.3119458+00 \\
.3136799+00 \\
.3148973+00 \\
.3156964+00 \\
.3161544+00\end{array}$ & \begin{tabular}{|l|}
$106-02$ \\
$030-02$ \\
$4451-02$ \\
$3141-03$ \\
$3822-03$
\end{tabular} & $\begin{array}{l}.1199243-05 \\
.1055014-05 \\
.9350005-06 \\
.8341128-06 \\
.7485251-06\end{array}$ & $\begin{array}{l}.5961402-08 \\
.4994208-08 \\
.4226632-08 \\
.3609619-08 \\
.3107905-08\end{array}$ & $\begin{array}{l}453935-01 \\
623158-01 \\
888212-01 \\
234250-01 \\
493054-02\end{array}$ & $\begin{array}{l}.1452532-02 \\
.1350668-02 \\
.1261182-02 \\
.1182007-02 \\
.1111502-02\end{array}$ & $\begin{array}{l}.2693583-05 \\
.2360614-05 \\
.2084720-05 \\
.1853704-05 \\
.1658437-05\end{array}$ & $\begin{array}{l}-.1849664-07 \\
-.1543994-07 \\
-.1302342-07 \\
-.1108781-07 \\
.9519107-08\end{array}$ & $\begin{array}{l}-.1849099+00 \\
-.1666508+00 \\
-.1504087+00 \\
-.1358749+00 \\
-.1228005+00\end{array}$ & $\begin{array}{l}.3303476-02 \\
.3065836-02 \\
.2857666-02 \\
.2673954-02 \\
.2510747-02\end{array}$ & $\begin{array}{l}-.8925483-05 \\
-.7798169-05 \\
-.6867264-05 \\
-.6090235-05 \\
-.5435370-05\end{array}$ & $\begin{array}{l}.7706617-07 \\
.6413628-07 \\
.5394714-07 \\
.4581006-07 \\
.3923371-07\end{array}$ \\
\hline $\begin{array}{l}44.000 \\
46.000 \\
48.000 \\
50.000 \\
52.000\end{array}$ & $\begin{array}{l}.3163330+00 \\
.3162816+00 \\
.3160400+00 \\
.3156408+00 \\
.3151108+00\end{array}$ & & $.6753167-06$ & & $\begin{array}{r}.1236256-02 \\
-.3508120-02 \\
-.7806670-02 \\
-.1171511-01 \\
-.1528042-01\end{array}$ & $\begin{array}{l}-.1048353-02 \\
-.9914972-03 \\
-.9400620-03 \\
-.8933278-03 \\
-.8506952-03\end{array}$ & $\begin{array}{l}.1491981-05 \\
.1348995-05 \\
.1225306-05 \\
.1117628-05 \\
.1023338-05\end{array}$ & $\begin{array}{l}-.8234256-08 \\
-.7171774-08 \\
-.6285491-08 \\
-.5540283-08 \\
-.4909106-08\end{array}$ & $\begin{array}{l}-.1109823+00 \\
-.1002529+00 \\
-.9047336-01 \\
-.8152709-01 \\
-.7331577-01\end{array}$ & $\begin{array}{l}.2364887-02 \\
.2233824-02 \\
.2115477-02 \\
.2008132-02 \\
.1910366-02\end{array}$ & $\begin{array}{l}-.4878656-05 \\
-.4401653-05 \\
-.3990022-05 \\
-.3632482-05 \\
-.3320064-05\end{array}$ & $\begin{array}{l}.3386129-0 \\
.2942945-0\end{array}$ \\
\hline $\begin{array}{l}54.000 \\
56.000 \\
58.000 \\
60.000 \\
64.000\end{array}$ & & & $-.4307152-06$ & & $\begin{array}{l}-.1854244-01 \\
-.2153522-01 \\
-.2428797-01 \\
-.2682593-01 \\
-.3134227-01\end{array}$ & & & & & & & \\
\hline $\begin{array}{l}68.000 \\
72.000 \\
76.000 \\
80.000 \\
84.000\end{array}$ & & $\begin{array}{l}.5055219-03 \\
.4718416-03 \\
.4420887-03 \\
.4156292-03 \\
.3919576-03\end{array}$ & $\begin{array}{l}-.2612010-06 \\
-.2309494-06 \\
-.2056312-06 \\
-.1842342-06 \\
-.1659922-06\end{array}$ & & $\begin{array}{l}-.3522822-01 \\
-.3859492-01 \\
-.4152947-01 \\
-.4410100-01 \\
-.4636508-01\end{array}$ & & & & & & & $.8721627-0$ \\
\hline $\begin{array}{l}88 \\
92 \\
9\end{array}$ & & $\begin{array}{l}.3339433-03 \\
.3180113-03 \\
.2999784-03\end{array}$ & $\begin{array}{l}-.1503168-06 \\
-.1367503-06 \\
-.1249321-06 \\
-.1145755-06 \\
-.1033381-06\end{array}$ & & $\begin{array}{l}-.4836682-01 \\
-.5014319-01 \\
-.5172478-01 \\
-.5313707-01 \\
-.5469665-01\end{array}$ & & & & $\begin{array}{l}.6930414-02 \\
.1163327-01 \\
.1589129-01 \\
.1976009-01 \\
.2411948-01\end{array}$ & & & $\begin{array}{l}.2698881-0 \\
.2330644-0\end{array}$ \\
\hline $\begin{array}{l}115.000 \\
120.000 \\
125.000 \\
130.000\end{array}$ & $\begin{array}{l}.2806011+00 \\
.2782019+00 \\
.2758627+00\end{array}$ & $\begin{array}{l}.2837516-03 \\
.2690786-03 \\
.2557516-03 \\
.2435976-03 \\
.2324719-03\end{array}$ & $\begin{array}{l}-.9367027-07 \\
-.8529370-07 \\
-.7798900-07 \\
-.7158136-07 \\
-.6593011-07\end{array}$ & & $\begin{array}{l}-.5605959-01 \\
-.5725427-01 \\
-.5830410-01 \\
-.5922856-01 \\
-.6004394-01\end{array}$ & & & & & & & $\begin{array}{l}.1562785-0 \\
.1383747-0 \\
.1231351-0\end{array}$ \\
\hline $\begin{array}{l}140.000 \\
145.000 \\
150.000 \\
160.000\end{array}$ & $\begin{array}{l}.2735829+00 \\
.2713613+00 \\
.2691966+00 \\
.2670874+00 \\
.2630288+00\end{array}$ & $\begin{array}{l}.2222523-03 \\
.2128351-03 \\
.2041315-03 \\
.1960652-03 \\
.1815898-03\end{array}$ & $\begin{array}{l}-.6092106-07 \\
-.5646071-07 \\
-.5247196-07 \\
-.4889075-07 \\
-.4274522-07\end{array}$ & $\begin{array}{l}.9651189-10 \\
.8686428-10 \\
.7848122-10 \\
.7116048-10 \\
.5907787-10\end{array}$ & $\begin{array}{l}-.6076399-01 \\
-.6140039-01 \\
-.6196307-01 \\
-.6246058-01 \\
-.6328851-01\end{array}$ & & & & $\begin{array}{l}.4250025-01 \\
.4465614-01 \\
.4662842-01 \\
.4843672-01 \\
.5162724-01\end{array}$ & $\begin{array}{l}.5839823-03 \\
.5586620-03 \\
.5352962-03 \\
.5136726-03 \\
.4749447-03\end{array}$ & $\begin{array}{l}-.3990212-06 \\
-.3688863-06 \\
-.3420139-06 \\
-.3179523-06 \\
-.2768157-06\end{array}$ & $\begin{array}{l}.9882823-0 \\
.8907810-0 \\
.8058547-0 \\
.6661891-0\end{array}$ \\
\hline $\begin{array}{l}180.000 \\
190.000 \\
200.000 \\
210.000\end{array}$ & $\begin{array}{l}.2520089+00 \\
.2486775+00 \\
.2454971+00\end{array}$ & $\begin{array}{l}.1578990-03 \\
.1480954-03 \\
.1393637-03 \\
.1315409-03\end{array}$ & $\begin{array}{l}-.2993614-07 \\
-.2692774-07 \\
-.2435124-07\end{array}$ & $\begin{array}{l}.4962276-10 \\
.4211281-10 \\
.3606863-10 \\
.3114655-10 \\
.2709564-10\end{array}$ & $\begin{array}{l}-.6393204-01 \\
-.6442764-01 \\
-.6480342-01 \\
-.6508140-01 \\
-.6527897-01\end{array}$ & $\begin{array}{l}-.1872605-03 \\
-.1755124-03 \\
-.1650592-03 \\
-.1557028-03\end{array}$ & $\begin{array}{l}.6931243-07 \\
.6186455-07 \\
.5555234-07 \\
.5015677-07\end{array}$ & $\begin{array}{l}-.1426044-09 \\
-.1207840-09 \\
-.1032596-09 \\
-.8901654-10 \\
-.7731587-10\end{array}$ & $\begin{array}{l}.5665936-01 \\
.5865428-01 \\
.6037746-01 \\
.6187160-01\end{array}$ & $\begin{array}{l}.4412849-03 \\
.4117818-03 \\
.3857274-03 \\
.3625643-03 \\
.3418475-03\end{array}$ & $\begin{array}{l}-.2431273-06 \\
-.2151998-06 \\
-.1917964-06 \\
-.1719941-06 \\
-.1550932-06\end{array}$ & $\begin{array}{l}.4713325-09 \\
.4023394-09 \\
.3463550-09 \\
.3004346-09\end{array}$ \\
\hline $\begin{array}{l}220.000 \\
240.000 \\
260.000 \\
280.000 \\
300.000 \\
\end{array}$ & $\begin{array}{l}.2424570+00 \\
.2367598+00 \\
.2315169+00 \\
.2266707+00 \\
.2221730+00\end{array}$ & $\begin{array}{l}.1021886-03 \\
.9362896-04 \\
.8631089-04 \\
\end{array}$ & $\begin{array}{l}1570785-07 \\
.1350011-07 \\
.1172805-07\end{array}$ & $\begin{array}{l}.2372976-10 \\
.1852568-10 \\
.1476119-10 \\
.1196725-10 \\
.9847703-11\end{array}$ & .6506393 & $\begin{array}{l}.1472831-03 \\
.1327537-03 \\
.1206729-03 \\
.1104819-03 \\
.1017784-03\end{array}$ & $\begin{array}{l}.4550904-07 \\
.3795785-07 \\
.3213912-07 \\
.2756160-07 \\
.2389636-07\end{array}$ & $\begin{array}{l}-.6761047-10 \\
-.5263930-10 \\
-.4184059-10 \\
-.3384674-10 \\
-.2779646-10\end{array}$ & $\begin{array}{l}.6529529-01 \\
.6692203-01 \\
.6817207-01 \\
.6913190-01\end{array}$ & $\begin{array}{l}.3232179-03 \\
.2911000-03 \\
.2644252-03 \\
.2419457-03 \\
.2227640-03\end{array}$ & $\begin{array}{l}-.1405556-06 \\
-.1169817-06 \\
-.9885953-07 \\
-.8463337-07 \\
-.7326421-07\end{array}$ & $\begin{array}{l}.1616954-0 \\
.1305664-0 \\
.1070515-0\end{array}$ \\
\hline
\end{tabular}




\begin{tabular}{|c|c|c|c|c|c|c|c|c|c|c|c|c|}
\hline$T^{*}$ & $\mathrm{~B}^{*(0)}$ & $\mathrm{B}^{*(1)}$ & $\mathrm{B}^{*(2)}$ & $\mathrm{B}^{*(3)}$ & $\mathrm{T}^{*} \frac{\partial \mathrm{B}^{*(0)}}{\partial \mathrm{T}^{*}}$ & $T^{*} \frac{\partial B^{*(1)}}{\partial T^{*}}$ & $\mathrm{~T}^{*} \frac{\partial \mathrm{B}^{*(2)}}{\partial \mathrm{T}^{*}}$ & $T^{*} \frac{\partial B^{*(3)}}{\partial T^{*}}$ & $\mathrm{~T}^{* 2} \frac{\partial^{2} \mathrm{~B}^{*(0)}}{\partial \mathrm{T}^{* 2}}$ & $T^{* 2} \frac{\partial^{2} B^{*(1)}}{\partial T^{* 2}}$ & $\mathrm{~T}^{* 2} \frac{\partial^{2} \mathrm{~B}^{*(2)}}{\partial \mathrm{T}^{* 2}}$ & $\mathrm{~T}^{* 2} \frac{\partial^{2} \mathrm{~B}^{*(3)}}{\partial \mathrm{T}^{* 2}}$ \\
\hline .300 & $381+02$ & $459+02$ & $2094497+02$ & $.2703724+02$ & $.9503545+02$ & $-.1058401+03$ & $.1375759+03$ & $-.2176730+03$ & $-.4415330+03$ & $.6941081+03$ & $-.1115171+04$ & $.2072022+04$ \\
\hline .400 & $-.1752750+02$ & $.6080189+01$ & $-.3619714+01$ & $.3080385+01$ & $.3768635+02$ & $-.2446648+02$ & $.2054056+02$ & $-.2183749+02$ & $-.1444092+03$ & $.1385325+03$ & $-.1469586+03$ & $.1857509+03$ \\
\hline .500 & $-.1119594+02$ & $.2629996+01$ & $-.1087318+01$ & $.6786947+00$ & $.2113078+02$ & $-.9217033+01$ & $.5570008+01$ & $-.4402419+01$ & $-.7133920+02$ & $.4705443+02$ & $-.3654083+02$ & $.3463112+02$ \\
\hline .600 & $-.8043546+01$ & $.1435070+01$ & $-.4426982+00$ & $.2162764+00$ & $.1407498+02$ & $-.4520301+01$ & $.2100225+01$ & $-.1312468+01$ & $-.4353453+02$ & $.2133467+02$ & $-.1291431+02$ & $.9738379+01$ \\
\hline .700 & $-.6180303+01$ & $.9012830+00$ & $-.2177729+00$ & $.8696065-01$ & $.1034877+02$ & $-.2605507+01$ & $.9726156+00$ & $-.5007705+00$ & $-.3005074+02$ & $.1155307+02$ & $-.5684819+01$ & $.3549172+01$ \\
\hline .750 & $-.5510663+01$ & $.7410238+00$ & $-.1606564+00$ & $.5870844-01$ & $.9097973+01$ & $-.2064286+01$ & $.6992919+00$ & $-.3306050+00$ & $-.2576154+02$ & $.8910852+01$ & $-.4000044+01$ & $.2297850+01$ \\
\hline .775 & $-.5221074+01$ & $.6768685+00$ & $-.1394067+00$ & $.4885674-01$ & $.8572180+01$ & $-.1853204+01$ & $.5995830+00$ & $-.2722832+00$ & $-.2399915+02$ & $.7900117+01$ & $-.3395514+01$ & $.1875409+01$ \\
\hline .800 & $-.4956506+01$ & $.6209620+00$ & $-.1217079+00$ & $.4096961-01$ & $.8099945+01$ & $-.1672184+01$ & $.5175181+00$ & $-.2260737+00$ & $-.2243806+02$ & $.7043512+01$ & $-.2902899+01$ & $.1543717+01$ \\
\hline .825 & $-.4713890+01$ & $.5719555+00$ & $-.1068571+00$ & $.3459934-01$ & $.7673790+01$ & $-.1515932+01$ & $.4494376+00$ & $-.1891197+00$ & $-.2104774+02$ & $.6312424+01$ & $-.2498106+01$ & $.1280748+01$ \\
\hline .850 & $-.4490634+01$ & $.5287624+00$ & $-.9430993-01$ & $.2941188-01$ & $.7287528+01$ & $-.1380240+01$ & $.3925416+00$ & $-.1593119+00$ & $-.1980327+02$ & $.5684399+01$ & $-.2162888+01$ & $.1070382+01$ \\
\hline .875 & $-.4284535+01$ & $.4904999+00$ & $-.8364122-01$ & $.2515529-01$ & $.6936001+01$ & $-.1261742+01$ & $.3446667+00$ & $-.1350748+00$ & $-.1868415+02$ & $.5141642+01$ & $-.1883277+01$ & $.9006827+00$ \\
\hline .900 & $-.4093710+01$ & $.4564450+00$ & & & & -.11577 & $.3041250+00$ & $-.1152194+00$ & $-.1767342+02$ & $.4669939+01$ & $-.1648474+01$ & $7180+00$ \\
\hline .925 & $-.3916541+01$ & $.4260019+00$ & $-.6666622-01$ & $.1871114-01$ & $.6320518+01$ & $-.1065953+01$ & $.2695885+00$ & $-.9883952-01$ & $-.1675691+02$ & $.4257836+01$ & $-.1450057+01$ & $.6497326+00$ \\
\hline .950 & $-.3751627+01$ & $.3986757+00$ & $-.5987949-01$ & $.1626133-01$ & $.6049800+01$ & $362+00$ & $035+00$ & $23804-01$ & $273+02$ & $.3896037+01$ & $-.1281399+01$ & $.5565701+00$ \\
\hline .975 & $-.3597753+01$ & $.3740531+00$ & $-.5398283-01$ & $.1419853-01$ & $.5800072+01$ & $-.9122690+00$ & $.2145274+00$ & $08-01$ & $-.1516085+02$ & $.3576943+01$ & $-.1137247+01$ & $.4792590+00$ \\
\hline 1.000 & $-.3453857+01$ & $.3517866+00$ & $-.4883598-01$ & $.1245203-01$ & $.5569055+01$ & $-.8476090+00$ & $.1924818+00$ & $-.6432458-01$ & $-.1446271+02$ & $.3294301+01$ & $-.1013399+01$ & $.4147148+00$ \\
\hline 1.020 & -.33 & $0+00$ & $-.4518053-01$ & & & & & & & & & \\
\hline 1.040 & $-.3242100+01$ & $.3203451+00$ & $-.4188191-01$ & .10175 & $529+01$ & $64+00$ & $098+00$ & $-.5201334-0 \mathrm{r}$ & $-.1346052+02$ & $.2905302+01$ & $-.8492869+00$ & $.3321671+00$ \\
\hline 1.060 & $-.3143885+01$ & $.3062947+00$ & $-.3889786-01$ & $.9237879-02$ & $.5079708+01$ & $19+00$ & $53+00$ & -.4 & $84+02$ & 01 & +00 & $.2984968+00$ \\
\hline 1.080 & $-.3050302+01$ & $.2932191+00$ & $-.3619195-01$ & $.8402640-02$ & $.4934205+01$ & $-.6814168+00$ & $.1391977+00$ & $-.4249164-01$ & $-.1257858+02$ & $.2579794+01$ & $-.7180786+00$ & $.2689299+00$ \\
\hline 1.100 & $-.2961036+01$ & $.2810290+00$ & $-.3373263-01$ & $.7659801-02$ & $.4796381+01$ & $-.6475966+00$ & $.1289999+00$ & $-.3854303-01$ & $-.1217645+02$ & $.2436736+01$ & $-.6623160+00$ & $.2428881+00$ \\
\hline 1.120 & & & $-.3149255-01$ & .695 & & & & & +02 & & & \\
\hline 1.140 & -.279 & .25899 & $-.2944789-01$ & 02 & $1+01$ & -.58 & -00 & 01 & $56+02$ & 01 & $699+00$ & $.1995100+00$ \\
\hline 1.160 & -.2716 & $.2490215+00$ & $-.2757787-01$ & $.5874590-02$ & $470+01$ & $66+00$ & $607+00$ & $-.2914428-01$ & $131+02$ & $223+01$ & $-.5255723+00$ & $.1814149+00$ \\
\hline 1.180 & $-.2641717+01$ & $.2396622+00$ & $-.2586426-01$ & $.5397954-02$ & $.4311104+01$ & $-.5350470+00$ & $.9681286-01$ & $-.2665968-01$ & $-.1078113+02$ & $.1967251+01$ & $-.4882833+00$ & $.1653037+00$ \\
\hline 1.200 & -.2570 & $.2308684+00$ & $-.2429108-01$ & $.4968877-02$ & $.4204022+01$ & $-.5115951+00$ & $.9046799-01$ & $-.2443325-01$ & $-.1047768+02$ & $.1870821+01$ & $-.4543793+00$ & $.1509239+00$ \\
\hline 1.220 & & & & & & & & -.2243365 & $-.1018973+02$ & $.1781237+01$ & $-.4234912+00$ & $.1380593+00$ \\
\hline 1.240 & -.24 & +00 & $7-01$ & .42318 & .40 & $076+00$ & -01 & $-.2063389-01$ & $-.9916178+01$ & $.1697881+01$ & $-.3952966+00$ & $.1265241+00$ \\
\hline 1.260 & -.2 & +00 & 01 & .391 & .39 & -.4 & .74 & $1060-01$ & $04+01$ & $203+01$ & $-.3695127+00$ & $.1161586+00$ \\
\hline 1.280 & -.231 & $.2005029+00$ & $-.1914465-01$ & $.3627203-02$ & $.3821850+01$ & $-.4320252+00$ & $.6996109-01$ & $-.1754354-01$ & $-.9408290+01$ & $.1547709+01$ & $-.3458914+00$ & $.1068245+00$ \\
\hline 1.300 & $-.2252824+01$ & $.1939367+00$ & -.1809 & $.3365644-02$ & $.3736412+01$ & $-.4151252+00$ & $.6582055-01$ & $-.1621511-01$ & $-.9172195+01$ & $.1479958+01$ & $-.3242140+00$ & $.9840225-01$ \\
\hline 1.320 & & & 01 & & 36 & - & & & $-.8946951+01$ & .14 & & $.9078798-01$ \\
\hline 1.340 & -.214 & +00 & -01 & -02 & .3575967 & $2725+00$ & .58 & $-.1391462-01$ & $-.8731853+01$ & $7137+01$ & $-.2859413+00$ & $.8389122-01$ \\
\hline 1.360 & -.20 & +00 & $3-01$ & $.2711369-02$ & $.3500552+01$ & $704+00$ & $127-01$ & $-.1291742-01$ & $-.8526255+01$ & $.1301390+01$ & $-.2690238+00$ & $.7763301-01$ \\
\hline 1.380 & -.2039 & $.1709367+00$ & $.1458614-01$ & $.2529513-02$ & $.3428094+01$ & $-.3568632+00$ & $.5217265-01$ & $-.1200800-01$ & $-.8329563+01$ & $.1249019+01$ & $-.2534005+00$ & $.7194427-01$ \\
\hline 1.400 & $-.1990231+01$ & $.1658930+00$ & $-.1385587-01$ & $.2362785-02$ & $.3358426+01$ & $-.3442922+00$ & $.4936185-01$ & $-.1117730-01$ & $-.8141229+01$ & .119976 & -.23895 & $667-2>$ \\
\hline 1.420 & & & & & & & & & 1 & .115338 & -.2 & .620401 \\
\hline 1.440 & $-.1897489+01$ & $.1565243+00$ & $-.1253770-01$ & $.2068926-02$ & $.3226849+01$ & $505+00$ & $924-01$ & - & $661+01$ & .1109667 & $-.2131610+00$ & $.5772462-01$ \\
\hline 1.460 & $-.1853411+01$ & .15 & -.1194 & $2-02$ & & & & $93-02$ & $530+01$ & .1068413 & $-.2016379+00$ & $.5377637-01$ \\
\hline 1.480 & $-.1810763+01$ & $.1480135+00$ & $-.1138447-01$ & $.1819778-02$ & $.3104703+01$ & $-.3003725+00$ & $.3994271-01$ & $-.8494664-02$ & $-.7461960+01$ & $.1029444+01$ & $-.1909244+00$ & $.5015877-01$ \\
\hline 1.500 & $-.1769478+01$ & $.1440465+00$ & $-.1086168-01$ & $.1709423-02$ & $.3046859+01$ & $-.2907703+00$ & $.3797030-01$ & $-.7954282-02$ & $-.7308581+01$ & $.9925968+00$ & $-.1809513+00$ & $.4683935-01$ \\
\hline & & & & & & & & $6915-02$ & & & & \\
\hline 1.580 & $-.1616757+01$ & $.1298410+00$ & $-.9068955-02$ & $.1344252-02$ & $.2834565+01$ & $-.2568412+00$ & $.3126704-01$ & $-.6179901-02$ & $-.6750475+01$ & .8636224 & $-.1473073+00$ & $.3601218-01$ \\
\hline 1.620 & $-.1547097+01$ & $.1236050+00$ & $-.8322331-02$ & & $.2738601+$ & & -01 & -.5 & $-.6500718+01$ & $330+00$ & $11+00$ & .317719 \\
\hline 1.660 & $-.1481404+01$ & $.1178625+00$ & $.7657314-02$ & $.1072553-02$ & $.2648593+01$ & $-.2288197+00$ & $.2606241-01$ & $-.4875717-02$ & $-.6267919+01$ & $.7586676+00$ & $-.1214865+00$ & .2813758-01 \\
\hline 1.700 & $-.1419352+01$ & $.1125612+00$ & $-.7062977-02$ & $.9627988-03$ & $.2564009+01$ & $-.2166021+00$ & $.2389322-01$ & $-.4353517-02$ & $-.6050449+01$ & $.7133914+00$ & $-.1108145+00$ & $.2500851-01$ \\
\hline & & & & & & & & & & & & \\
\hline 1.780 & $-.1305046+01$ & $.1031051+00$ & $-.6050899-02$ & $.7828696-03$ & $.2409280+01$ & $-.1951075+00$ & $.2023327-01$ & $-.3504220-02$ & $-.5655940+01$ & $.6345136+00$ & $-.9294435-01$ & $.1995425-01$ \\
\hline 1.820 & $-.1252297+01$ & $.9887558-01$ & $-.5618706-02$ & $.7089129-03$ & $.2338342+01$ & $-.1856232+00$ & $.1868459-01$ & $-.3157904-02$ & $-.5476525+01$ & $.6000455+00$ & $-.8543963-01$ & $.1790742-01$ \\
\hline 1.860 & $-.1202195+01$ & .9493623-01 & $|-.5227849-02|$ & .6436253-03 & $.2271232+01$ & $-.1768650+00$ & $.1729206-01$ & $-.2853702-02$ & $-.5307641+01$ & $.5684104+00$ & $-.7872345-01$ & $.1611720-01$ \\
\hline
\end{tabular}


$(m=9)$

\begin{tabular}{|c|c|c|c|c|c|c|c|c|c|c|c|c|}
\hline $\mathrm{T}^{*}$ & $\mathrm{~B}^{*(0)}$ & $B^{*(1)}$ & $\mathrm{B}^{*(2)}$ & $\mathrm{B}^{*(3)}$ & $\mathrm{T}^{*} \frac{\partial \mathrm{B}^{*(0)}}{\partial \mathrm{T}^{*}}$ & $\mathrm{~T}^{*} \frac{\partial \mathrm{B}^{*(1)}}{\partial \mathrm{T}^{*}}$ & $T^{*} \frac{\partial B^{*(2)}}{\partial T^{*}}$ & $\mathrm{~T}^{*} \frac{\partial \mathrm{B}^{*(3)}}{\partial \mathrm{T}^{*}}$ & $T^{* 2} \frac{\partial^{2} B^{*(0)}}{\partial T^{* 2}}$ & $T^{* 2} \frac{\partial^{2} B^{*(1)}}{\partial T^{* 2}}$ & $\mathrm{~T}^{* 2} \frac{\partial^{2} \mathrm{~B}^{*(2)}}{\partial \mathrm{T}^{* 2}}$ & $T^{* 2} \frac{\partial^{2} B^{*(3)}}{\partial T^{* 2}}$ \\
\hline $\begin{array}{l}1.900 \\
1.940 \\
1.980 \\
2.020 \\
2.060\end{array}$ & $\begin{array}{l}.1154549+01 \\
.1109186+01 \\
.1065949+01 \\
.1024695+01 \\
.9852936+00\end{array}$ & $\begin{array}{l}.9126003-01 \\
.8782307-01 \\
.8460418-01 \\
.8158449-01 \\
.7874716-01\end{array}$ & $\begin{array}{l}-.4873457-02 \\
-.4551332-02 \\
-.4257844-02 \\
-.3989847-02 \\
-.3744600-02\end{array}$ & $\begin{array}{l}.5858073-03 \\
.5344493-03 \\
.4886976-03 \\
.4478280-03 \\
.4112232-03\end{array}$ & $\begin{array}{l}.2207650+01 \\
.2147326+01 \\
.2090018+01 \\
.2035508+01 \\
.1983595+01\end{array}$ & $\begin{array}{l}-.1687597+00 \\
-.1612430+00 \\
-.1542583+00 \\
-.1477559+00 \\
-.1416914+00\end{array}$ & $\begin{array}{l}.1603647-01 \\
.1490135-01 \\
.1387257-01 \\
.1293792-01 \\
.1208685-01\end{array}$ & $\begin{array}{l}-.2585603-02 \\
-.2348573-02 \\
-.2138376-02 \\
-.1951437-02 \\
-.1784725-02\end{array}$ & $\begin{array}{l}-.5148404+01 \\
-.4998029+01 \\
-.4855807+01 \\
-.4721107+01 \\
-.4593356+01\end{array}$ & $\begin{array}{l}.5393071+00 \\
.5124729+00 \\
.4876781+00 \\
.4647209+00 \\
.4434241+00\end{array}$ & $\begin{array}{l}-.7269541-01 \\
-.6726998-01 \\
-.6237398-01 \\
-.5794460-01 \\
-.5392771-01\end{array}$ & $\begin{array}{l}.1454600-01 \\
.1316248-01 \\
.1194038-01 \\
.1085764-01 \\
.9895622-02\end{array}$ \\
\hline $\begin{array}{l}2.100 \\
2.140 \\
2.180 \\
2.220 \\
2.260\end{array}$ & $\begin{array}{l}-.9476245+00 \\
-.9115787+00 \\
-.8770556+00 \\
-.8439630+00 \\
-.8122157+00\end{array}$ & $\begin{array}{l}.7607716-01 \\
.7356097-01 \\
.7118644-01 \\
.6894264-01 \\
.6681967-01\end{array}$ & $\begin{array}{l}-.3519710-02 \\
-.3313082-02 \\
-.3122876-02 \\
-.2947470-02 \\
-.2785432-02\end{array}$ & $\begin{array}{l}.3783557-03 \\
.3487728-03 \\
.3220845-03 \\
.2979544-03 \\
.2760909-03\end{array}$ & $\begin{array}{l}.1934100+01 \\
.1886859+01 \\
.1841722+01 \\
.1798553+01 \\
.1757225+01\end{array}$ & $\begin{array}{l}-.1360258+00 \\
-.1307241+00 \\
-.1257551+00 \\
-.1210910+00 \\
-.1167068+00\end{array}$ & $\begin{array}{l}.1131019-01 \\
.1059994-01 \\
.9949117-02 \\
.9351609-02 \\
.8802033-02\end{array}$ & $\begin{array}{l}-.1635658-02 \\
-.1502033-02 \\
-.1381960-02 \\
-.1273814-02 \\
-.1176195-02\end{array}$ & $\begin{array}{l}-.4472040+01 \\
-.4356692+01 \\
-.4246890+01 \\
-.4142248+01 \\
-.4042416+01\end{array}$ & $\begin{array}{l}.4236308+00 \\
.4052025+00 \\
.3880158+00 \\
.3719612+00 \\
.3569407+00\end{array}$ & $\begin{array}{l}-.5027651-01 \\
-.4695039-01 \\
-.4391401-01 \\
-.4113654-01 \\
-.3859097-01\end{array}$ & $\begin{array}{l}.9038515-02 \\
.8272880-02 \\
.7587237-02 \\
.6971750-02 \\
.6417966-02\end{array}$ \\
\hline $\begin{array}{l}2.300 \\
2.340 \\
2.380 \\
2.420 \\
2.460\end{array}$ & $\begin{array}{l}-.7817352+00 \\
-.7524490+00 \\
-.7242899+00 \\
-.6971957+00 \\
-.6711087+00\end{array}$ & $\begin{array}{l}.6480858-01 \\
.6290126-01 \\
.6109033-01 \\
.5936905-01 \\
.5773131-01\end{array}$ & $\begin{array}{l}-.2635496-02 \\
-.2496535-02 \\
-.2367548-02 \\
-.2247642-02 \\
-.2136020-02\end{array}$ & & & & & & & & $\begin{array}{l}-.3625359-01 \\
-.3410356-01 \\
-.3212244-01 \\
-.3029397-01 \\
-.2860369-01\end{array}$ & $\begin{array}{l}.5918502-02 \\
.5467325-02 \\
.5058692-02 \\
.4687938-02 \\
.4350912-0\end{array}$ \\
\hline $\begin{array}{l}2.500 \\
2.600 \\
2.700 \\
2.800 \\
2.900\end{array}$ & $\begin{array}{l}-.5869934+00 \\
-.5329750+00 \\
-.4833354+00 \\
-.4375776+00\end{array}$ & $\begin{array}{l}.5617148-01 \\
.5258021-01 \\
.4937568-01 \\
.4650150-01 \\
.4391141-01\end{array}$ & $\begin{array}{l}-.2031967-02 \\
-.1800895-02 \\
-.1604893-02 \\
-.1437449-02 \\
-.1293461-02\end{array}$ & & & & & & $\begin{array}{l}-.3528316+01 \\
-.3349278+01 \\
-.3186778+01 \\
-.3038644+01 \\
-.2903069+01\end{array}$ & $\begin{array}{l}.2842085+00 \\
.2607120+00 \\
.2402134+00 \\
.2222169+00 \\
.2063252+00\end{array}$ & $\begin{array}{l}-.2703876-01 \\
-.2360280-01 \\
-.2073412-01 \\
-.1831994-01 \\
-.1627331-01\end{array}$ & $\begin{array}{l}.4043985-02 \\
.3388410-02 \\
.2861946-02 \\
.2435118-02 \\
.2086022-02\end{array}$ \\
\hline $\begin{array}{l}3.000 \\
3.100 \\
3.200 \\
3.300 \\
3.400\end{array}$ & $\begin{array}{l}-.3952766+00 \\
-.3560670+00 \\
-.3196326+00 \\
-.2856988+00 \\
-.2540257+00\end{array}$ & $\begin{array}{l}.4156719-01 \\
.3943697-01 \\
.3749401-01 \\
.3571574-01 \\
.3408296-01\end{array}$ & $\begin{array}{l}-.1168889-02 \\
-.1060509-02 \\
-.9657254-03 \\
-.8824286-03 \\
-.8088952-03\end{array}$ & & & & & & $\begin{array}{l}-.2778531+01 \\
-.2663745+01 \\
-.2557615+01 \\
-.2459204+01 \\
-.2367704+01\end{array}$ & $\begin{array}{l}.1922171+00 \\
.1796304+00 \\
.1683498+00 \\
.1581969+00 \\
.1490227+00\end{array}$ & $\begin{array}{l}-.1452653-01 \\
-.1302633-01 \\
-.1173041-01 \\
-.1060490-01 \\
-.9622479-02\end{array}$ & $\begin{array}{l}.1359034-0 \\
.1190615-0 \\
.1047953-0\end{array}$ \\
\hline $\begin{array}{l}3.500 \\
3.600 \\
3.700 \\
3.800 \\
3.900\end{array}$ & $\begin{array}{l}-.2244029+00 \\
-.1966450+00 \\
-.1705883+00 \\
-.1460874+00 \\
-.1230129+00\end{array}$ & $\begin{array}{l}.3257929-01 \\
.3119064-01 \\
.2990485-01 \\
.2871136-01 \\
.2760100-01\end{array}$ & $\begin{array}{l}-.7437058-03 \\
-.6856844-03 \\
-.6338505-03 \\
-.5873822-03 \\
-.5455865-03\end{array}$ & $\begin{array}{l}.4608589-04 \\
.4125465-04 \\
.3705980-04 \\
.3340169-04 \\
.3019860-04\end{array}$ & $\begin{array}{r}.1003148+01 \\
.9677382+00 \\
.9344711+00 \\
.9031582+00 \\
.8736326+00\end{array}$ & & & & $\begin{array}{l}-.2282414+01 \\
-.2202726+01 \\
-.2128109+01 \\
-.2058093+01 \\
-.1992269+01\end{array}$ & & $\begin{array}{l}-.8760911-02 \\
-.8002004-02 \\
-.7330763-02 \\
-.6734756-02 \\
-.6203616-02\end{array}$ & $\begin{array}{l}.8223479-0 \\
.7327748-0 \\
.6553227-0 \\
.5880561-0\end{array}$ \\
\hline $\begin{array}{l}4.000 \\
4.100 \\
4.200 \\
4.300 \\
4.400\end{array}$ & $\begin{array}{l}-.1012493+00 \\
-.8069299-01 \\
-.6125092-01 \\
-.4283928-01 \\
-.2538237-01\end{array}$ & $\begin{array}{l}.2656572-01 \\
.2559845-01 \\
.2469299-01 \\
.2384381-01 \\
.2304604-01\end{array}$ & \begin{tabular}{|l|}
$-.5078768-03$ \\
$-.4737535-03$ \\
$-.4427896-03$ \\
$-.4146184-03$ \\
$-.3889240-03$
\end{tabular} & & & $\begin{array}{l}-.3681121-01 \\
-.3537629-01 \\
-.3403647-01\end{array}$ & & $\begin{array}{l}-.1056487-03 \\
-.9570586-04 \\
-.8693858-04 \\
-.7918151-04 \\
-.7229601-04\end{array}$ & $\begin{array}{l}-.1930271+01 \\
-.1871777+01 \\
-.1816499+01 \\
-.1764178+01 \\
-.1714584+01\end{array}$ & $\begin{array}{l}.1087213+00 \\
.1037799+00 \\
.9920764-01 \\
.9496729-01 \\
.9102642-01\end{array}$ & $\begin{array}{l}-.5728651-02 \\
-.5302538-02 \\
-.4919075-02 \\
-.4572986-02 \\
-.4259765-02\end{array}$ & $\begin{array}{l}.5293925-0 \\
.4780304-0 \\
.4328937-0 \\
.3930881-0 \\
.3578664-0\end{array}$ \\
\hline $\begin{array}{l}4.500 \\
4.600 \\
4.700 \\
4.800 \\
4.900\end{array}$ & $\begin{array}{r}-.8811610-02 \\
.6935219-02 \\
.2191473-01 \\
.3617852-01 \\
.4977375-01\end{array}$ & $\begin{array}{l}.2229533-01 \\
.2158779-01 \\
.2091995-01 \\
.2028868-01 \\
.1969117-01\end{array}$ & $\begin{array}{l}-.3654329-03 \\
-.3439076-03 \\
-.3241410-03 \\
-.3059522-03 \\
-.2891824-03\end{array}$ & $\begin{array}{l}.1745265-04 \\
.1605946-04 \\
.1480772-04 \\
.1368017-04 \\
.1266202-04\end{array}$ & & $\begin{array}{l}-.3278313-01 \\
-.3160859-01 \\
-.3050605-01 \\
-.2946943-01 \\
-.2849330-01\end{array}$ & & & $\begin{array}{l}-.1667510+01 \\
-.1622768+01 \\
-.1580191+01 \\
-.1539624+01 \\
-.1500928+01\end{array}$ & $\begin{array}{l}.8735645-01 \\
.8393221-01 \\
.8073141-01 \\
.7773427-01 \\
.7492320-01\end{array}$ & $\begin{array}{l}-.3975545-02 \\
-.3716997-02 \\
-.3481243-02 \\
-.3265786-02 \\
-.3068452-02\end{array}$ & $\begin{array}{l}.2987655-0 \\
.2739106-0 \\
.2516566-0 \\
.2316792-0\end{array}$ \\
\hline $\begin{array}{l}5.000 \\
5.100 \\
5.200 \\
5.300 \\
5.400\end{array}$ & $\begin{array}{l}.6274355-01 \\
.7512749-01 \\
.8696191-01 \\
.9828027-01 \\
.1091134+00\end{array}$ & $\begin{array}{l}.1858753-01 \\
.1807702-01 \\
.1759148-01 \\
.1712918-01\end{array}$ & $\begin{array}{l}-.2736920-03 \\
-.2593577-03 \\
-.2460705-03 \\
-.2337337-03 \\
-.2222612-03\end{array}$ & $\begin{array}{l}.1090459-04 \\
.1014471-04 \\
.9452523-05 \\
.8820763-05\end{array}$ & $\begin{array}{l}.6172723+00 \\
.6016943+00 \\
.5867526+00 \\
.5724088+00\end{array}$ & $\begin{array}{l}-.2757279-01 \\
-.2670353-01 \\
-.2588156-01 \\
-.2510334-01 \\
-.2436562-01\end{array}$ & $\begin{array}{l}.7034767-03 \\
.6654362-03 \\
.6302319-03 \\
.5975980-03\end{array}$ & $\begin{array}{l}-.4385024-04 \\
-.4061710-04 \\
-.3768654-04 \\
-.3502460-04 \\
-.3260171-04\end{array}$ & $\begin{array}{l}-.1428658+01 \\
-.1394863+01 \\
-.1362495+01 \\
-.13314666+01\end{array}$ & $\begin{array}{l}.7228247-01 \\
.6979804-01 \\
.6745727-01 \\
.6524884-01 \\
.6316251-01\end{array}$ & $\begin{array}{l}-.2887345-02 \\
-.2720801-02 \\
-.2567360-02 \\
-.2425736-02 \\
-.2294792-02\end{array}$ & $\begin{array}{l}.1974819-0 \\
.1828176-0 \\
.1695294-0 \\
.1574629-0\end{array}$ \\
\hline $\begin{array}{l}5.500 \\
5.600 \\
5.800 \\
6.000 \\
6.200\end{array}$ & $\begin{array}{l}.1194897+00 \\
.1294356+00 \\
.1481315+00 \\
.1653758+00 \\
.1813192+00\end{array}$ & $\begin{array}{l}.1668855-01 \\
.1626816-01 \\
.1548295-01 \\
.1476428-01 \\
.1410432-01\end{array}$ & $\left|\begin{array}{l}-.2115763-03 \\
-.2016105-03 \\
-.1835972-03 \\
-.1678011-03 \\
-.1538815-03\end{array}\right|$ & $\begin{array}{l}.8243069-05 \\
.7713854-05 \\
.6781791-05 \\
.5991767-05 \\
.5318029-05\end{array}$ & $\begin{array}{l}.5586281+00 \\
.5453778+00 \\
.5203512+00 \\
.4971145+00 \\
.4754830+00\end{array}$ & $\begin{array}{l}-.2366548-01 \\
-.2300028-01 \\
-.2176517-01 \\
-.2064334-01 \\
-.1962057-01\end{array}$ & $\begin{array}{l}.5672987-03 \\
.5391239-03 \\
.4884171-03 \\
.4442007-03 \\
.4054446-03\end{array}$ & $\begin{array}{l}-.3039208-04 \\
-.2837316-04 \\
-.2483064-04 \\
-.2184248-04 \\
-.1930590-04\end{array}$ & $\begin{array}{l}-.1301695+01 \\
-.1273108+01 \\
-.1219210+01 \\
-.1169280+01 \\
-.1122895+01\end{array}$ & $\begin{array}{l}.6118904-01 \\
.5932007-01 \\
.5586596-01 \\
.5274729-01 \\
.4992007-01\end{array}$ & $\left|\begin{array}{l}-.2173521-02 \\
-.2061029-02 \\
-.1859287-02 \\
-.1684166-02 \\
-.1531335-02\end{array}\right|$ & $\begin{array}{l}.1189669-03 \\
.1042601-03 \\
.9182488-0\end{array}$ \\
\hline
\end{tabular}


$(m=9)$

\begin{tabular}{|c|c|c|c|c|c|c|c|c|c|c|c|c|}
\hline $\mathrm{T}^{*}$ & $\mathrm{~B}^{*(0)}$ & $B^{*(1)}$ & $\mathrm{B}^{*(2)}$ & $\mathrm{B}^{*(3)}$ & $\mathrm{T}^{*} \frac{\partial \mathrm{B}^{*(0)}}{\partial \mathrm{T}^{*}}$ & $\mathrm{~T}^{*} \frac{\partial \mathrm{B} *(1)}{\partial \mathrm{T}^{*}}$ & $\mathrm{~T}^{*} \frac{\partial \mathrm{B}^{*(2)}}{\partial \mathrm{T}^{*}}$ & $\mathrm{~T}^{*} \frac{\partial \mathrm{B}^{*(3)}}{\partial \mathrm{T}^{*}}$ & $T^{* 2} \frac{\partial^{2} B^{*(0)}}{\partial T^{* 2}}$ & $T^{* 2} \frac{\partial^{2} B^{*(1)}}{\partial T^{* 2}}$ & $\mathrm{~T}^{* 2} \frac{\partial^{2} \mathrm{~B}^{*(2)}}{\partial \mathrm{T}^{* 2}}$ & $\mathrm{~T}^{* 2} \frac{\partial^{2} \mathrm{~B}^{*(3)}}{\partial \mathrm{T}^{* 2}}$ \\
\hline $\begin{array}{l}6.400 \\
6.600 \\
6.800 \\
7.000 \\
7.200\end{array}$ & $\begin{array}{l}.1960928+00 \\
.2098108+00 \\
.2225734+00 \\
.2344688+00 \\
.2455746+00\end{array}$ & $\begin{array}{l}.1349639-01 \\
.1293475-01 \\
.1241450-01 \\
.1193135-01 \\
.1148160-01\end{array}$ & $\begin{array}{l}-.1415593-03 \\
-.1306050-03 \\
-.1208280-03 \\
-.1120691-03 \\
-.1041950-03\end{array}$ & $\begin{array}{l}.4740182-05 \\
.4241940-05 \\
.3810198-05 \\
.3434338-05 \\
.3105694-05\end{array}$ & $\begin{array}{l}.4552963+00 \\
.4364149+00 \\
.4187166+00 \\
.4020940+00 \\
.3864525+00\end{array}$ & $\begin{array}{l}-.1868486-01 \\
-.1782604-01 \\
-.1703539-01 \\
-.1630546-01 \\
-.1562980-01\end{array}$ & $\begin{array}{l}.3713111-03 \\
.3411141-03 \\
.3142880-03 \\
.2903630-03 \\
.2689469-03\end{array}$ & \begin{tabular}{|}
$-.1713991-04$ \\
$-.1528016-04$ \\
$-.1367512-04$ \\
$-.1228322-04$ \\
$-.1107067-04$
\end{tabular} & $\begin{array}{l}-.1079691+01 \\
-.1039351+01 \\
-.1001600+01 \\
-.9661963+00 \\
-.9329271+00\end{array}$ & $\begin{array}{l}.4734741-01 \\
.4499820-01 \\
.4284605-01 \\
.4086841-01 \\
.3904597-01\end{array}$ & $\begin{array}{l}-.1397289-02 \\
-.1279171-02 \\
-.1174635-02 \\
-.1081742-02 \\
-.9988792-03\end{array}$ & $\begin{array}{l}.8124626-04 \\
.7219586-04 \\
.6441180-04 \\
.5768361-04 \\
.5184092-04\end{array}$ \\
\hline $\begin{array}{l}7.400 \\
7.600 \\
7.800 \\
8.000 \\
8.200\end{array}$ & $\begin{array}{l}.2559600+00 \\
.2656862+00 \\
.2748081+00 \\
.2833745+00 \\
.2914294+00\end{array}$ & $\begin{array}{l}.1106202-01 \\
.1066975-01 \\
.1030230-01 \\
.9957465-02 \\
.9633271-02\end{array}$ & $-.7469411-04$ & & $\begin{array}{l}.3717080+00 \\
.3577859+00 \\
.3446198+00 \\
.3321498+00 \\
.3203228+00\end{array}$ & $\begin{array}{l}-.1500282-01 \\
-.1441968-01 \\
-.1387610-01 \\
-.1336836-01 \\
-.1289318-01\end{array}$ & $\begin{array}{l}.2497106-03 \\
.2323760-03 \\
.2167071-03 \\
.2025030-03 \\
.1895913-03\end{array}$ & $\begin{array}{l}-.1000989-04 \\
-.9078135-05 \\
-.8256625-05 \\
-.7529725-05 \\
-.6884361-05\end{array}$ & $\begin{array}{l}-.9016052+00 \\
-.8720645+00 \\
-.8441572+00 \\
-.8177515+00 \\
-.7927293+00\end{array}$ & $\begin{array}{l}.3736206-01 \\
.3580224-01 \\
.3435396-01 \\
.3300626-01 \\
.3174950-01\end{array}$ & $\begin{array}{l}-.9246991-03 \\
-.8580670-03 \\
-.7980244-03 \\
-.7437567-03 \\
-.6945690-03\end{array}$ & $\begin{array}{l}.4674496-04 \\
.4228193-04 \\
.3835801-04 \\
.3489538-04 \\
.3182918-04\end{array}$ \\
\hline $\begin{array}{l}8.400 \\
8.600 \\
8.800 \\
9.000 \\
9.200\end{array}$ & $\begin{array}{l}.2990125+00 \\
.3061594+00 \\
.3129022+00 \\
.3192705+00 \\
.3252906+00\end{array}$ & & $\begin{array}{l}.5905499-04 \\
.5589011-04\end{array}$ & & $\begin{array}{l}.3090904+00 \\
.2984093+00 \\
.2882401+00 \\
.2785472+00 \\
.2692982+00\end{array}$ & & & & & & & $\begin{array}{l}.2910508-04 \\
.2667733-04 \\
.2450726-04 \\
.2256204-04 \\
.2081368-04\end{array}$ \\
\hline $\begin{array}{r}9.400 \\
9.600 \\
9.800 \\
10.000 \\
10.200\end{array}$ & $\begin{array}{l}.3309868+00 \\
.3363811+00 \\
.3414939+00 \\
.3463434+00 \\
.3509468+00\end{array}$ & & $.4323665-04$ & & $\begin{array}{l}.2604635+00 \\
.2520162+00 \\
.2439314+00 \\
.2361868+00 \\
.2287613+00\end{array}$ & $\begin{array}{l}-.1058342-01 \\
-.1027028-01 \\
-.9973562-02 \\
-.9692047-02 \\
-.9424642-02\end{array}$ & & & & & & $\begin{array}{l}.1652655-04 \\
.1535727-04 \\
.1429392-04\end{array}$ \\
\hline & $\begin{array}{l}.3634291+00 \\
.3671914+00 \\
.3741877+00\end{array}$ & $\begin{array}{l}.7039282-02 \\
.6866921-02 \\
.6702199-02 \\
.6544634-02 \\
.6249241-02\end{array}$ & $-.3289524-04$ & & $\begin{array}{l}.2216359+00 \\
.2147930+00 \\
.2082163+00 \\
.2018908+00 \\
.1899383+00\end{array}$ & $\begin{array}{l}-.9170352-02 \\
-.8928270-02 \\
-.8697570-02 \\
-.8477498-02 \\
-.8066528-02\end{array}$ & $\begin{array}{l}.1016930-03 \\
.9682174-04 \\
.9227902-04 \\
.8803664-04 \\
.8035436-04\end{array}$ & $\begin{array}{l}-.2944682-05 \\
-.2753642-05 \\
-.2578576-05 \\
-.2417866-05 \\
-.2133965-05\end{array}$ & $\begin{array}{l}-.5846640+00 \\
-.5702744+00 \\
-.5564475+00 \\
-.5431511+00 \\
-.5180322+00\end{array}$ & & & \\
\hline $\begin{array}{l}12.200 \\
12.600 \\
13.000 \\
13.400\end{array}$ & $\begin{array}{l}.3916129+00 \\
.3964354+00 \\
.4008467+00\end{array}$ & $\begin{array}{l}.5977598-02 \\
.5727028-02 \\
.5495234-02 \\
.5280237-02 \\
.5080324-02\end{array}$ & $\begin{array}{l}-.2392034-04 \\
-.2223851-04\end{array}$ & $\begin{array}{l}.4999257-06 \\
.4484744-06 \\
.4038112-06 \\
.3648581-06\end{array}$ & $\begin{array}{l}.1788357+00 \\
.1684967+00 \\
.1588462+00 \\
.1498185+00 \\
.1413563+00\end{array}$ & $\begin{array}{l}-.7345250-02 \\
-.7027304-02 \\
-.6733642-02 \\
-.6461674-02\end{array}$ & $\begin{array}{l}.7360161-04 \\
.6763738-04 \\
.6234602-04 \\
.5763203-04 \\
.5341607-04\end{array}$ & & & & $\begin{array}{l}-.2605519-03 \\
-.2387533-03 \\
-.2194749-03 \\
-.2023519-03 \\
-.1870820-03\end{array}$ & $\begin{array}{l}.8473368-05 \\
.7527102-05 \\
.6714893-05 \\
.6014044-05 \\
.5406285-05\end{array}$ \\
\hline $\begin{array}{l}14.600 \\
15.000 \\
16.000\end{array}$ & $\begin{array}{l}.4119916+00 \\
.4151145+00 \\
.4218646+00\end{array}$ & $\begin{array}{l}.4894002-02 \\
.4719966-02 \\
.4557072-02 \\
.4404311-02 \\
.4061126-02\end{array}$ & $-.1457849-04$ & $\begin{array}{l}.3307354-06 \\
.3007204-06 \\
.2742158-06 \\
.2507252-06 \\
.2026234-06\end{array}$ & $\begin{array}{l}.1188843+00 \\
.1122319+00 \\
.9712897-01\end{array}$ & $\begin{array}{l}-.6209158-02 \\
-.5974147-02 \\
-.5754939-02 \\
-.5550042-02 \\
-.5092165-02\end{array}$ & $\begin{array}{l}.4963176-04 \\
.4622327-04 \\
.4314341-04 \\
.4035205-04 \\
.3442288-04\end{array}$ & $\begin{array}{l}-.1102613-05 \\
-.1000014-05 \\
-.9096612-06 \\
-.8297932-06 \\
-.6669358-06\end{array}$ & $\begin{array}{l}-.3992458+00 \\
-.3835222+00 \\
-.3686976+00 \\
-.3546975+00 \\
-.3228845+00\end{array}$ & & $\begin{array}{l}-.1734134-03 \\
-.1611348-03 \\
-.1500682-03 \\
-.1400628-03 \\
-.1188938-03\end{array}$ & $\begin{array}{l}.4876800-05 \\
.4413495-05 \\
.4006436-05 \\
.3647412-05 \\
.2917949-05\end{array}$ \\
\hline $\begin{array}{l}19.000 \\
20.000 \\
21.000\end{array}$ & $\begin{array}{l}.4354250+00 \\
.4383549+00 \\
.4407116+00\end{array}$ & $\begin{array}{l}.3764500-02 \\
.3505767-02 \\
.3278261-02 \\
.3076775-02 \\
.2897184-02\end{array}$ & $.1 / 30438-03$ & & $\begin{array}{l}.8389259-01 \\
.7220388-01 \\
.6181245-01 \\
.5251896-01 \\
.4416276-01\end{array}$ & $\begin{array}{l}-.4699195-02 \\
-.4358605-02 \\
-.4060848-02 \\
-.3798533-02 \\
-.3565852-02\end{array}$ & $\begin{array}{l}.2967863-04 \\
.2582806-04 \\
.2266332-04 \\
.2003305-04 \\
.1782506-04\end{array}$ & $\begin{array}{l}-.5438330-06 \\
-.4491127-06 \\
-.3750791-06 \\
-.3164022-06 \\
-.2693126-06\end{array}$ & $\begin{array}{l}-.2949610+00 \\
-.2702592+00 \\
-.2482558+00 \\
-.2285350+00 \\
-.2107623+00\end{array}$ & $\begin{array}{l}.1090375-01 \\
.1007873-01 \\
.9360928-02 \\
.8731334-02 \\
.8175111-02\end{array}$ & $\begin{array}{l}-.1020463-03 \\
-.8843972-04 \\
-.7730733-04 \\
-.6809388-04 \\
-.6038979-04\end{array}$ & $\begin{array}{l}.2369251-05 \\
.1948958-05 \\
.1621816-05 \\
.1363527-05 \\
.1156983-05\end{array}$ \\
\hline $\begin{array}{l}22.000 \\
23.000 \\
24.000 \\
25.000 \\
26.000\end{array}$ & $\begin{array}{l}.4425889+00 \\
.4440629+00 \\
.4451956+00 \\
.4460383+00 \\
.4466335+00\end{array}$ & $\begin{array}{l}.2736183-02 \\
.2591090-02 \\
.2459712-02 \\
.2340234-02 \\
.2231146-02\end{array}$ & $\begin{array}{l}-.5185837-05 \\
-.4743922-05\end{array}$ & $\begin{array}{l}48456-07 \\
83981-07 \\
23487-07\end{array}$ & $\begin{array}{l}.3661294-01 \\
.2976175-01 \\
.2351971-01 \\
.1781192-01 \\
.1257518-01\end{array}$ & $\begin{array}{l}-.3358181-02 \\
-.3171799-02 \\
-.3003675-02 \\
-.2851323-02 \\
-.2712678-02\end{array}$ & $\begin{array}{l}.1595484-04 \\
.1435780-04 \\
.1298393-04 \\
.1179408-04 \\
.1075722-04\end{array}$ & $\begin{array}{l}-.2310957-06 \\
-.1997630-06 \\
-.1738364-06 \\
-.1522017-06 \\
-.1340086-06\end{array}$ & $\begin{array}{l}-.1946657+00 \\
-.1800212+00 \\
-.1666433+00 \\
-.1543767+00 \\
-.1430904+00\end{array}$ & $\begin{array}{l}.7680513-02 \\
.7238136-02 \\
.6840365-02 \\
.6480971-02 \\
.6154816-02\end{array}$ & $\begin{array}{l}-.5388796-04 \\
-.4835475-04 \\
-.4360998-04 \\
-.3951307-04 \\
-.3595307-04\end{array}$ & $\begin{array}{l}.9899137-06 \\
.8533665-06 \\
.7407090-06 \\
.6469596-06 \\
.5683282-06\end{array}$ \\
\hline $\begin{array}{l}27.000 \\
28.000 \\
29.000 \\
30.000 \\
32.000\end{array}$ & $\begin{array}{l}.4472171+00 \\
.4472604+00 \\
.4471679+00 \\
.4466452+00\end{array}$ & $\begin{array}{l}.2131179-02 \\
.2039261-02 \\
.1954478-02 \\
.1876049-02 \\
.1735654-02\end{array}$ & $\begin{array}{l}-.4355349-05 \\
-.4011950-05 \\
-.3707051-05 \\
-.3435158-05 \\
-.2972929-05\end{array}$ & $\begin{array}{l}.2990508-07 \\
.2687930-07 \\
.2195337-07\end{array}$ & $\begin{array}{l}.7755798-02 \\
.3307896-02 \\
.8079828-03 \\
.4625947-02 \\
.1148291-01\end{array}$ & $\begin{array}{l}-.2586018-02 \\
-.2469893-02 \\
-.2363075-02 \\
-.2264517-02 \\
-.2088712-02\end{array}$ & $\begin{array}{l}.9848544-05 \\
.9048038-05 \\
.8339406-05 \\
.7709282-05 \\
.6642257-05\end{array}$ & $\begin{array}{l}-.1186005-06 \\
-.1054656-06 \\
-.9420033-07 \\
-.8448417-07 \\
-.6872063-07\end{array}$ & $\begin{array}{l}-.1326732+00 \\
-.1230301+00 \\
-.1140795+00 \\
-.1057507+00 \\
-.9072116-01\end{array}$ & $\begin{array}{l}.5857624-02 \\
.5585813-02 \\
.5336355-02 \\
.5106681-02 \\
.4698202-02\end{array}$ & $\begin{array}{l}-.3284157-04 \\
-.3010742-04 \\
-.2769290-04 \\
-.2555082-04 \\
-.2193499-04\end{array}$ & $\begin{array}{l}.5018979-06 \\
.4453997-06 \\
.3970509-06 \\
.3554383-06 \\
.2881215-06\end{array}$ \\
\hline
\end{tabular}


$(m=9)$

\begin{tabular}{|c|c|c|c|c|c|c|c|c|c|c|c|c|}
\hline $\mathrm{T}^{*}$ & $\mathrm{~B}^{*(0)}$ & *(1) & $\mathrm{B}^{*(2)}$ & $\mathrm{B}^{*(3)}$ & $T^{*} \frac{\partial B^{*(0)}}{\partial T^{*}}$ & $T^{*} \frac{\partial B^{*(1)}}{\partial T^{*}}$ & $\mathrm{~T}^{*} \frac{\partial \mathrm{B}^{*(2)}}{\partial \mathrm{T}^{*}}$ & $\mathrm{~T}^{*} \frac{\partial \mathrm{B}^{*(3)}}{\partial \mathrm{T}^{*}}$ & $T^{* 2} \frac{\partial^{2} B^{*(0)}}{\partial T^{* 2}}$ & $\mathrm{~T}^{* 2} \frac{\partial^{2} \mathrm{~B}^{*(1)}}{\partial \mathrm{T}^{* 2}}$ & $T^{* 2} \frac{\partial^{2} B^{*(2)}}{\partial T^{* 2}}$ & $\mathrm{~T}^{* 2} \frac{\partial^{2} \mathrm{~B}^{*(3)}}{\partial \mathrm{T} * 2}$ \\
\hline $\begin{array}{l}34.000 \\
36.000 \\
38.000 \\
40.000 \\
42.000\end{array}$ & $\begin{array}{l}.4457657+00 \\
.4446162+00 \\
.4432621+00 \\
.4417531+00 \\
.4401279+00\end{array}$ & $\begin{array}{l}.1613697-02 \\
.1506841-02 \\
.1412498-02 \\
.1328638-02 \\
.1253638-02\end{array}$ & $\begin{array}{l}.2597044-05 \\
.2287423-05 \\
.2029469-05 \\
.1812376-05 \\
.1628006-05\end{array}$ & $\begin{array}{l}.1816511-07 \\
.1520392-07 \\
.1285564-07 \\
.1096935-07 \\
.9436634-08\end{array}$ & $\begin{array}{l}-.1745827-01 \\
-.2270324-01 \\
-.2733668-01 \\
-.3145332-01 \\
-.3512951-01\end{array}$ & $\begin{array}{l}-.1936660-02 \\
-.1803959-02 \\
-.1687218-02 \\
-.1583785-02 \\
-.1491558-02\end{array}$ & $\begin{array}{l}.5778850-05 \\
.5070880-05 \\
.4483521-05 \\
.3991117-05 \\
.3574441-05\end{array}$ & $\begin{array}{l}-.5665040-07 \\
-.4725301-07 \\
-.3982810-07 \\
-.3388427-07 \\
-.2906988-07\end{array}$ & $\begin{array}{l}-.7753734-01 \\
-.6588615-01 \\
-.5552127-01 \\
-.4624632-01 \\
-.3790277-01\end{array}$ & $\begin{array}{l}.4346201-02 \\
.4040008-02 \\
.3771443-02 \\
.3534143-02 \\
.3323079-02\end{array}$ & $\begin{array}{l}-.1902086-04 \\
-.1664011-04 \\
-.1467160-04 \\
-.1302649-04 \\
-.1163843-04\end{array}$ & $\begin{array}{l}.2367660-06 \\
.1969174-06 \\
.1655306-06 \\
.1404770-06 \\
.1202384-06\end{array}$ \\
\hline $\begin{array}{l}44.000 \\
46.000 \\
48.000 \\
50.000 \\
52.000\end{array}$ & & & $\begin{array}{l}1470143-05 \\
.1333970-05 \\
1215715-05 \\
1112385-05 \\
1021585-05\end{array}$ & $\begin{array}{l}.8178240-08 \\
.7135319-08 \\
.6263556-08 \\
.5529147-08 \\
.4906001-08\end{array}$ & $\begin{array}{l}-.3842751-01 \\
-.4139853-01 \\
-.4408510-01 \\
-.4652282-01 \\
-.4874168-01\end{array}$ & $\begin{array}{l}-.1408850-02 \\
-.1334294-02 \\
-.1266767-02 \\
.1205342-02 \\
.1149247-02\end{array}$ & $\begin{array}{l}.3218868-05 \\
.2913118-05 \\
.2648380-05 \\
.2417698-05 \\
.2215522-05\end{array}$ & $\begin{array}{l}-.2512884-07 \\
-.2187167-07 \\
-.1915613-07 \\
-.1687406-07 \\
-.1494219-07\end{array}$ & $\begin{array}{l}-.3036129-01 \\
-.2351528-01 \\
-.1727615-01 \\
-.1156961-01 \\
-.6332967-02\end{array}$ & & $\begin{array}{l}-.1045713-04 \\
-.9443916-05 \\
-.8568701-05 \\
-.7807781-05 \\
-.7142294-05\end{array}$ & $\begin{array}{l}.1037125-06 \\
.9008619-07 \\
.7875075-07 \\
.6924438-07 \\
.6121259-07\end{array}$ \\
\hline $\begin{array}{l}54.000 \\
56.000 \\
58.000 \\
60.000 \\
64.000\end{array}$ & $\begin{array}{l}.4254684+00 \\
.4236001+00 \\
.4199024+00\end{array}$ & & & $\begin{array}{l}73761-08 \\
16388-08 \\
21125-08 \\
77742-08 \\
15072-08\end{array}$ & $\begin{array}{l}-.5076715-01 \\
-.5262097-01 \\
-.5432183-01 \\
-.5588585-01 \\
-.5865756-01\end{array}$ & & $\begin{array}{l}.2037379-05 \\
.1879637-05 \\
.1739322-05 \\
.1613976-05 \\
.1400371-05\end{array}$ & $\begin{array}{l}.1329577-07 \\
.1188388-07 \\
.1066612-07 \\
.9610189-08 \\
.7884406-08\end{array}$ & $\begin{array}{r}-.1512904-02 \\
.2936185-02 \\
.7053544-02 \\
.1087312-01 \\
.1773326-01\end{array}$ & & $\begin{array}{l}-.6557087-05 \\
-.6039879-05 \\
-.5580632-05 \\
-.5171084-05 \\
-.4474807-05\end{array}$ & $\begin{array}{l}.5438019-07 \\
.4853129-07 \\
.4349500-07 \\
.3913489-07 \\
.3202441-07\end{array}$ \\
\hline $\begin{array}{l}68.000 \\
72.000 \\
76.000 \\
80.000 \\
84.000\end{array}$ & $\begin{array}{l}.4162734+00 \\
.4127259+00 \\
.4092675+00 \\
.4059020+00 \\
.4026306+00\end{array}$ & & & & $\begin{array}{l}-.66 \\
-.67\end{array}$ & & $\begin{array}{l}45-05 \\
41-05 \\
16-06 \\
85-06 \\
55-06\end{array}$ & $\begin{array}{l}633-08 \\
352-08 \\
401-08 \\
967-08 \\
648-08\end{array}$ & & $\begin{array}{l}.1828572-02 \\
.1705399-02 \\
.1596836-02 \\
.1500484-02 \\
.1414438-02\end{array}$ & $\begin{array}{l}-.3908580-05 \\
-.3442173-05 \\
-.3053605-05 \\
-.2726601-05 \\
-.2448902-05\end{array}$ & $\begin{array}{l}.2654422-07 \\
.2225211-07 \\
.1884227-07 \\
.1609878-07 \\
.1386621-07\end{array}$ \\
\hline $\begin{array}{r}88.000 \\
92.000 \\
96.000 \\
100.000 \\
105.000\end{array}$ & $\begin{array}{l}.3963675+00 \\
.3933721+00 \\
.3904641+00 \\
.3869474+00\end{array}$ & & & & $\begin{array}{l}-.7163279-01 \\
-.7250787-01\end{array}$ & & $\begin{array}{l}.6363947-06 \\
.5807491-06 \\
.5320495-06 \\
.4792815-06\end{array}$ & $\begin{array}{l}-.2996990-08 \\
-.2621525-08 \\
-.2306783-08 \\
-.2040919-08 \\
-.1763554-08\end{array}$ & & $\begin{array}{l}.1337162-02 \\
.1267410-02 \\
.1204158-02 \\
.1146558-02 \\
.1081435-02\end{array}$ & $\begin{array}{l}-.2211142-05 \\
-.2006066-05 \\
-.1827987-05 \\
-.1672397-05 \\
-.1504103-05\end{array}$ & $\begin{array}{l}.1203065-07 \\
.1050742-07 \\
.9232682-08 \\
.8157570-08 \\
.7037772-08\end{array}$ \\
\hline & & & $\begin{array}{l}-.1728080-06 \\
-.1587091-06 \\
-.1462661-06\end{array}$ & & $\begin{array}{l}-.7446304-01 \\
-.7494171-01 \\
-.7535288-01\end{array}$ & & $\begin{array}{l}.4339491-06 \\
.3947240-06 \\
.3605612-06 \\
.3306289-06 \\
.3042591-06\end{array}$ & & & & $\begin{array}{l}-.1359789-05 \\
-.1235132-05 \\
-.1126738-05 \\
-.1031911-05 \\
-.9484893-06\end{array}$ & $\begin{array}{l}.4706226-08 \\
.4163476-08 \\
.3701824-08\end{array}$ \\
\hline $\begin{array}{l}145.000 \\
150.000 \\
160.000\end{array}$ & $\begin{array}{l}.3628496+00 \\
.3602604+00 \\
.3553134+00\end{array}$ & $\begin{array}{l}.3069797-03 \\
.2948169-03 \\
.2835311-03 \\
.2632430-03\end{array}$ & $\begin{array}{l}-.1352298-06 \\
-.1253964-06 \\
-.1165974-06 \\
-.1086930-06 \\
-.9511794-07\end{array}$ & $\begin{array}{l}.2853987-09 \\
.2566097-09 \\
.2316161-09 \\
.2098086-09 \\
.1738603-09\end{array}$ & $\begin{array}{l}-.7570526-01 \\
-.7600625-01 \\
-.7626213-01 \\
-.7647829-01 \\
-.7680940-01\end{array}$ & & & & & & & $\begin{array}{l}.2966236-08 \\
.2671498-08 \\
.2414944-08 \\
.1993414-08\end{array}$ \\
\hline $\begin{array}{l}190.000 \\
200.000 \\
210.000\end{array}$ & $\begin{array}{l}.3462427+00 \\
.3420689+00 \\
.3381074+00 \\
.3343402+00\end{array}$ & $\begin{array}{l}.2299320-03 \\
.2161044-03 \\
.2037651-03 \\
.1926902-03\end{array}$ & $\begin{array}{l}-.8393691-07 \\
-.7461890-07 \\
-.6677221-07 \\
-.6010282-07 \\
-.5438659-07\end{array}$ & $\begin{array}{l}.1235017-09 \\
.1056026-09 \\
.9104730-10 \\
.7908487-10\end{array}$ & $\begin{array}{l}-.7702970-01 \\
-.7716206-01 \\
-.7722403-01 \\
-.7722932-01 \\
-.7718870-01\end{array}$ & $\begin{array}{l}-.2638710-03 \\
-.2477985-03 \\
-.2334728-03 \\
-.2206289-03\end{array}$ & $\begin{array}{l}.1534736-06 \\
.1370966-06 \\
.1232043-06 \\
.1113193-06\end{array}$ & $\begin{array}{l}-.4232484-09 \\
-.3579415-09 \\
-.3055616-09 \\
-.2630427-09 \\
-.2281560-09\end{array}$ & $\begin{array}{l}.7408844-01 \\
.7545928-01 \\
.7662324-01 \\
.7761453-01 \\
.7846061-01\end{array}$ & $\begin{array}{l}.6104601-03 \\
.5707648-03 \\
.5356494-03 \\
.5043796-03 \\
.4763684-03\end{array}$ & $\begin{array}{l}-.5351286-06 \\
-.4741170-06 \\
-.4229367-06 \\
-.3795901-06 \\
-.3425614-06\end{array}$ & $\begin{array}{l}.1406389-08 \\
.1198916-08 \\
.1030772-08 \\
.8929961-09\end{array}$ \\
\hline $\begin{array}{l}240.000 \\
260.000 \\
280.000 \\
300.000\end{array}$ & $\begin{array}{l}.3240512+00 \\
.3179107+00 \\
.3122518+00 \\
.3070109+00\end{array}$ & $.1282085-03$ & $\begin{array}{l}.4140280-07 \\
.3517549-07 \\
.3025799-07 \\
.2630705-07\end{array}$ & $\begin{array}{l}.5384026-10 \\
.4278647-10 \\
.3460149-10 \\
.2840582-10 \\
\end{array}$ & $\begin{array}{l}-.7711073-01 \\
-.7686896-01 \\
-.7654483-01 \\
-.7616570-01 \\
-.7575026-01 \\
\end{array}$ & $\begin{array}{l}.1890308-03 \\
.1723363-03 \\
.1582162-03 \\
.1461274-03 \\
\end{array}$ & $\begin{array}{r}909-07 \\
3058-07 \\
2260-07 \\
403-07 \\
\end{array}$ & $\begin{array}{l}-.1547421-09 \\
-.1227058-09 \\
-.9903975-10 \\
-.8116234-10 \\
\end{array}$ & $\begin{array}{l}.7918366-01 \\
.8033005-01 \\
.8116433-01 \\
.8176368-01 \\
.8218336-01 \\
\end{array}$ & $\begin{array}{l}.4511414-03 \\
.4075580-03 \\
.3712637-03 \\
.3406011-03 \\
.3143756-03 \\
\end{array}$ & $\begin{array}{l}-.3106835-06 \\
-.2589310-06 \\
-.2190891-06 \\
-.1877718-06 \\
-.1627138-06 \\
\end{array}$ & $\begin{array}{l}.6037489-09 \\
.4778814-09 \\
.3850921-09 \\
.3151118-09 \\
\end{array}$ \\
\hline
\end{tabular}


$(m=12)$

\begin{tabular}{|c|c|c|c|c|c|c|c|c|c|c|c|c|}
\hline $\mathrm{T}^{*}$ & B & 1) & 2) & 3) & $* \frac{\partial \mathrm{B}^{*(0)}}{\partial \mathrm{T}^{*}}$ & $\mathrm{~T}^{*} \frac{\partial \mathrm{B}^{*(1)}}{\partial \mathrm{T}^{*}}$ & $\mathrm{~T}^{*} \frac{\partial \mathrm{B}^{*(2)}}{\partial \mathrm{T}^{*}}$ & $\mathrm{~T}^{*} \frac{\partial \mathrm{B}^{*(3)}}{\partial \mathrm{T}^{*}}$ & $T^{* 2} \frac{\partial^{2} B^{*(0)}}{\partial T^{* 2}}$ & $T^{* 2} \frac{\partial^{2} B^{*(1)}}{\partial T^{* 2}}$ & $\mathrm{~T}^{* 2} \frac{\partial^{2} \mathrm{~B}^{*(2)}}{\partial \mathrm{T}^{* 2}}$ & $T^{* 2} \frac{\partial^{2} B^{*(3)}}{\partial T^{* 2}}$ \\
\hline $\begin{array}{l}.300 \\
.400 \\
.500 \\
.600 \\
.700\end{array}$ & $\begin{array}{l}-.2788058+02 \\
-.1379884+02 \\
-.8720205+01 \\
-.6197971+01 \\
-.4710037+01\end{array}$ & $\begin{array}{l}.2495831+02 \\
.7019887+01 \\
.3051606+01 \\
.1672560+01 \\
.1054610+01\end{array}$ & $\begin{array}{l}-.3392134+02 \\
-.5906112+01 \\
-.1785792+01 \\
-.7313444+00 \\
-.3616675+00\end{array}$ & $\begin{array}{r}.6183245+02 \\
.7112688+01 \\
.1579906+01 \\
.5070168+00 \\
.2051313+00\end{array}$ & $\begin{array}{l}.7660726+02 \\
.3026708+02 \\
.1692369+02 \\
.1124885+02 \\
.8257115+01\end{array}$ & $\begin{array}{l}-.1211236+03 \\
-.2810116+02 \\
-.1062264+02 \\
-.5226202+01 \\
-.3021161+01\end{array}$ & $\begin{array}{l}.2219993+03 \\
.3335049+02 \\
.9092938+01 \\
.3445280+01 \\
.1602553+01\end{array}$ & $\begin{array}{l}-.4958473+03 \\
-.5017340+02 \\
-.1018874+02 \\
-.3056777+01 \\
-.1172858+01\end{array}$ & $\begin{array}{l}-.3568768+03 \\
-.1163660+03 \\
-.5733952+02 \\
-.3491869+02 \\
-.2406266+02\end{array}$ & $\begin{array}{l}.7929995+03 \\
.1587144+03 \\
.5405480+02 \\
.2457106+02 \\
.1333733+02\end{array}$ & $\begin{array}{l}-.1795084+04 \\
-.2378388+03 \\
-.5941938+02 \\
-.2108967+02 \\
-.9319556+01\end{array}$ & $\begin{array}{l}.4705947+04 \\
.4252021+03 \\
.7980465+02 \\
.2257221+02 \\
.8269089+01\end{array}$ \\
\hline $\begin{array}{l}.750 \\
.775 \\
.800 \\
.825 \\
.850\end{array}$ & $\begin{array}{l}-.4175928+01 \\
-.3945069+01 \\
-.3734225+01 \\
-.3540935+01 \\
-.3363119+01\end{array}$ & $\begin{array}{l}.8686635+00 \\
.7941485+00 \\
.7291720+00 \\
.6721787+00 \\
.6219150+00\end{array}$ & $\begin{array}{l}-.2674671+00 \\
-.2323654+00 \\
-.2031005+00 \\
-.1785209+00 \\
-.1577345+00\end{array}$ & $\begin{array}{l}.1388823+00 \\
.1157352+00 \\
.9718115-01 \\
.8217750-01 \\
.6994541-01\end{array}$ & $\begin{array}{l}.7254013+01 \\
.6832553+01 \\
.6454140+01 \\
.6112751+01 \\
.5803406+01\end{array}$ & $\begin{array}{l}-.2396856+01 \\
-.2153180+01 \\
-.1944108+01 \\
-.1763553+01 \\
-.1606684+01\end{array}$ & $\begin{array}{l}.1154577+01 \\
.9909381+00 \\
.8561437+00 \\
.7442278+00 \\
.6506231+00\end{array}$ & $\begin{array}{l}-.7763107+00 \\
-.6401557+00 \\
-.5321575+00 \\
-.4456972+00 \\
-.3758826+00\end{array}$ & $\begin{array}{l}-.2061311+02 \\
-.1919640+02 \\
-.1794190+02 \\
-.1682495+02 \\
-.1582546+02\end{array}$ & $\begin{array}{l}.1029868+02 \\
.9135545+01 \\
.8149382+01 \\
.7307384+01 \\
.6583797+01\end{array}$ & $\begin{array}{l}-.6569467+01 \\
-.5581520+01 \\
-.4775874+01 \\
-.4113379+01 \\
-.3564363+01\end{array}$ & $\begin{array}{l}.5366455+01 \\
.4384905+01 \\
.3613422+01 \\
.3001166+01 \\
.2510901+01\end{array}$ \\
\hline $\begin{array}{l}.875 \\
.900 \\
.925 \\
.950 \\
.975\end{array}$ & $\begin{array}{l}-.3199015+01 \\
-.3047114+01 \\
-.2906119+01 \\
-.2774910+01 \\
-.2652514+01\end{array}$ & $\begin{array}{l}.5773619+00 \\
.5376846+00 \\
.5021948+00 \\
.4703204+00 \\
.4415835+00\end{array}$ & $\begin{array}{l}-.1400435+00 \\
-.1248975+00 \\
-.1118581+00 \\
-.1005741+00 \\
-.9076144-01\end{array}$ & $\begin{array}{l}.5989685-01 \\
.5158333-01 \\
.4465956-01 \\
.3885733-01 \\
.3396659-01\end{array}$ & $\begin{array}{l}.5521956+01 \\
.5264918+01 \\
.5029352+01 \\
.4812761+01 \\
.4613008+01\end{array}$ & $\begin{array}{l}-.1469627+01 \\
-.1349257+01 \\
-.1243026+01 \\
-.1148847+01 \\
-.1064998+01\end{array}$ & $\begin{array}{c}.5717978+00 \\
.5049953+00 \\
.4480448+00 \\
.3992229+00 \\
.3571510+00\end{array}$ & $\begin{array}{l}-.3190567+00 \\
-.2724570+00 \\
-.2339760+00 \\
-.2019913+00 \\
-.1752427+00\end{array}$ & $\begin{array}{l}-.1492689+02 \\
-.1411557+02 \\
-.1338007+02 \\
-.1271081+02 \\
-.1209970+02\end{array}$ & $\begin{array}{l}.5958211+01 \\
.5414313+01 \\
.4938958+01 \\
.4521473+01 \\
.4153131+01\end{array}$ & \begin{tabular}{|}
$-.3106099+01$ \\
$-.2721011+01$ \\
$-.2395379+01$ \\
$-.2118404+01$ \\
$-.1881518+01$
\end{tabular} & $\begin{array}{l}.2115033+01 \\
.1792892+01 \\
.1528833+01 \\
.1310906+01 \\
.1129899+01\end{array}$ \\
\hline $\begin{array}{l}1.000 \\
1.020 \\
1.040 \\
1.060 \\
1.080\end{array}$ & $\begin{array}{l}-.2538081+01 \\
-.2451766+01 \\
-.2369731+01 \\
-.2291669+01 \\
-.2217301+01\end{array}$ & $\begin{array}{l}.4155824+00 \\
.3965210+00 \\
.3788421+00 \\
.3624134+00 \\
.3471184+00\end{array}$ & $\begin{array}{l}-.8218929-01 \\
-.7609649-01 \\
-.7059485-01 \\
-.6561464-01 \\
-.6109571-01\end{array}$ & & \begin{tabular}{l|}
$.4428262+01$ \\
$.4290198+01$ \\
$.4160009+01$ \\
$.4037058+01$ \\
$.3920774+01$
\end{tabular} & $\begin{array}{l}-.9900469+00 \\
-.9356827+00 \\
-.8857221+00 \\
-.8397090+00 \\
-.7972439+00\end{array}$ & & $\begin{array}{l}-.1527442+00 \\
-.1372767+00 \\
-.1237052+00 \\
-.1117598+00 \\
-.1012141+00\end{array}$ & $\begin{array}{l}-.1153985+02 \\
-.1112492+02 \\
-.1073641+02 \\
-.1037200+02 \\
-.1002962+02\end{array}$ & $\begin{array}{l}.3826750+01 \\
.3591823+01 \\
.3377346+01 \\
.3181082+01 \\
.3001083+01\end{array}$ & & $\begin{array}{l}.9786521+00 \\
.8752701+00 \\
.7850083+00 \\
.7059429+00 \\
.6364680+00\end{array}$ \\
\hline $\begin{array}{l}1.100 \\
1.120 \\
1.140 \\
1.160 \\
1.180\end{array}$ & $\begin{array}{c}-.2146374+01 \\
-.2078658+01 \\
-.2013943+01 \\
-.1952035+01 \\
-.1892758+01\end{array}$ & $\begin{array}{l}.3328534+00 \\
.3195268+00 \\
.3070564+00 \\
.2953694+00 \\
.2844001+00\end{array}$ & $\begin{array}{l}-.5698601-01 \\
-.5324035-01 \\
-.4981935-01 \\
-.4668863-01 \\
-.4381805-01\end{array}$ & & \begin{tabular}{l|}
$.3810642+01$ \\
$.3706199+01$ \\
$.3607024+01$ \\
$.3512739+01$ \\
$.3422999+01$
\end{tabular} & $\begin{array}{l}-.7579757+00 \\
-.7215953+00 \\
-.6878293+00 \\
-.6564356+00 \\
-.6271992+00\end{array}$ & & $\begin{array}{l}-.9187724-01 \\
-.8358793-01 \\
-.7620918-01 \\
-.6962433-01 \\
-.6373367-01\end{array}$ & & $\begin{array}{l}.2835651+01 \\
.2683297+01 \\
.2542714+01 \\
.2412751+01 \\
.2292390+01\end{array}$ & & $\begin{array}{l}.5752370+00 \\
.5211167+00 \\
.4731497+00 \\
.4305239+00 \\
.3925486+00\end{array}$ \\
\hline $\begin{array}{l}1.200 \\
1.220 \\
1.240 \\
1.260 \\
1.280\end{array}$ & $\begin{array}{c}-.1835949+01 \\
-.1781460+01 \\
-.1729153+01 \\
-.1678902+01 \\
-.1630589+01\end{array}$ & $\begin{array}{l}.2740898+00 \\
.2643858+00 \\
.2552403+00 \\
.2466103+00 \\
.2384569+00\end{array}$ & $\begin{array}{l}-.4118116-01 \\
-.3875463-01 \\
-.3651788-01 \\
-.3445269-01 \\
-.3254290-01\end{array}$ & & \begin{tabular}{l|}
$.3337489+01$ \\
$.3255924+01$ \\
$.3178042+01$ \\
$.3103605+01$ \\
$.3032393+01$
\end{tabular} & $\begin{array}{l}-.5999282+00 \\
-.5744519+00 \\
-.5506170+00 \\
-.5282863+00 \\
-.5073365+00\end{array}$ & & $\begin{array}{l}-.5845169-01 \\
-.5370487-01 \\
-.4942976-01 \\
-.4557148-01 \\
-.4208240-01\end{array}$ & $\begin{array}{l}-.8347000+01 \\
-.8116508+0.1 \\
-.7897567+01 \\
-.7689364+01 \\
-.7491158+01\end{array}$ & $\begin{array}{l}.2180731+01 \\
.2076974+01 \\
.1980406+01 \\
.1890393+01 \\
.1806367+01\end{array}$ & $\begin{array}{l}-.7565068+00 \\
-.7054479+00 \\
-.6588208+00 \\
-.6161620+00 \\
-.5770641+00\end{array}$ & $\begin{array}{l}.3586341+00 \\
.3282751+00 \\
.3010377+00 \\
.2765479+00 \\
.2544825+00\end{array}$ \\
\hline $\begin{array}{l}1.300 \\
1.320 \\
1.340 \\
1.360 \\
1.380\end{array}$ & $\begin{array}{l}-.1584105+01 \\
-.1539350+01 \\
-.1496231+01 \\
-.1454661+01 \\
-.1414558+01\end{array}$ & $\begin{array}{l}.2307448+00 \\
.2234419+00 \\
.2165190+00 \\
.2099494+00 \\
.2037089+00\end{array}$ & $\begin{array}{l}-.3077412-01 \\
-.2913354-01 \\
-.2760972-01 \\
-.2619242-01 \\
-.2487244-01\end{array}$ & & \begin{tabular}{l|}
$.2964204+01$ \\
$.2898854+01$ \\
$.2836173+01$ \\
$.2776002+01$ \\
$.2718196+01$
\end{tabular} & $\begin{array}{l}-.4876565+00 \\
-.4691459+00 \\
-.4517142+00 \\
-.4352793+00 \\
-.4197665+00\end{array}$ & & $\begin{array}{l}-.3892111-01 \\
-.3605145-01 \\
-.3344186-01 \\
-.3106463-01 \\
-.2889545-01\end{array}$ & $\begin{array}{l}-.7302275+01 \\
-.7122096+01 \\
-.6950054+01 \\
-.6785631+01 \\
-.6628348+01\end{array}$ & $\begin{array}{l}.1727819+01 \\
.1654292+01 \\
.1585375+01 \\
.1520697+01 \\
.1459924+01\end{array}$ & $\begin{array}{l}-.5411685+00 \\
-.5081584+00 \\
-.4777532+00 \\
-.4497044+00 \\
-.4237907+00\end{array}$ & $\begin{array}{l}.2345616+00 \\
.2165418+00 \\
.2002111+00 \\
.1853844+00 \\
.1718996+00\end{array}$ \\
\hline $\begin{array}{l}1.400 \\
1.420 \\
1.440 \\
1.460 \\
1.480\end{array}$ & $\begin{array}{l}-.1375848+01 \\
-.1338460+01 \\
-.1302330+01 \\
-.1267394+01 \\
-.1233597+01\end{array}$ & $\begin{array}{l}.1977752+00 \\
.1921279+00 \\
.1867483+00 \\
.1816192+00 \\
.1767249+00\end{array}$ & $\begin{array}{l}-.2364155-01 \\
-.2249231-01 \\
-.2141804-01 \\
-.2041267-01 \\
-.1947075-01\end{array}$ & & $\begin{array}{l}.2609152+01 \\
.2557673+01 \\
.2508077+01 \\
.2460264+01\end{array}$ & $\begin{array}{l}-.4051083+00 \\
-.3912428+00 \\
-.3781139+00 \\
-.3656702+00 \\
-.3538646+00\end{array}$ & & $\begin{array}{l}-.2691289-01 \\
-.2509806-01 \\
-.2343424-01 \\
-.2190662-01 \\
-.2050207-01\end{array}$ & $\begin{array}{l}-.6477768+01 \\
-.6333484+01 \\
-.6195123+01 \\
-.6062339+01 \\
-.5934813+01\end{array}$ & $\begin{array}{l}.1402752+01 \\
.1348907+01 \\
.1298140+01 \\
.1250223+01 \\
.1204950+01\end{array}$ & $\begin{array}{l}-.3998153+00 \\
-.3776023+00 \\
-.3569946+00 \\
-.3378512+00 \\
-.3200458+00\end{array}$ & $\begin{array}{l}.1596146+00 \\
.1484043+00 \\
.1381586+00 \\
.1287801+00 \\
.1201827+00\end{array}$ \\
\hline $\begin{array}{l}1.500 \\
1.540 \\
1.580 \\
1.620 \\
1.660\end{array}$ & $\begin{array}{l}-.1200883+01 \\
-.1138509+01 \\
-.1079904+01 \\
-.1024742+01 \\
-.9727319+00\end{array}$ & $\begin{array}{l}.1720507+00 \\
.1633099+00 \\
.1553010+00 \\
.1479417+00 \\
.1411613+00\end{array}$ & $\begin{array}{l}-.1858731-01 \\
-.1697829-01 \\
-.1555428-01 \\
-.1428923-01 \\
-.1316140-01\end{array}$ & $\begin{array}{l}.4169406-02 \\
.3694723-02 \\
.3286610-02 \\
.2934116-02 \\
.2628338-02\end{array}$ & $\begin{array}{l}.2414140+01 \\
.2326621+01 \\
.2244890+01 \\
.2168400+01 \\
.2096665+01\end{array}$ & $\begin{array}{l}-.3426541+00 \\
-.3218635+00 \\
-.3030191+00 \\
-.2858836+00 \\
-.2702550+00\end{array}$ & $\begin{array}{l}.6418001-01 \\
.5820327-01 \\
.5295278-01 \\
.4832164-01 \\
.4422121-01\end{array}$ & $\begin{array}{l}-.1920888-01 \\
-.1691601-01 \\
-.1495727-01 \\
-.1327588-01 \\
-.1182599-01\end{array}$ & $\begin{array}{l}-.5812247+01 \\
-.5580918+01 \\
-.5366373+01 \\
-.5166900+01 \\
-.4981004+01\end{array}$ & $\begin{array}{l}.1162133+01 \\
.1083191+01 \\
.1012186+01 \\
.9480973+00 \\
.8900628+00\end{array}$ & $\begin{array}{l}-.3034645+00 \\
-.2735749+00 \\
-.2474766+00 \\
-.2245916+00 \\
-.2044437+00\end{array}$ & $\begin{array}{l}.1122900+00 \\
.9835469-01 \\
.8651556-01 \\
.7640667-01 \\
.6773420-01\end{array}$ \\
\hline $\begin{array}{l}1.700 \\
1.740 \\
1.780 \\
1.820 \\
1.860\end{array}$ & $\left|\begin{array}{r}-.9236164+00 \\
-.8771644+00 \\
-.8331688+00 \\
-.7914434+00 \\
-.7518202+00\end{array}\right|$ & $\begin{array}{l}.1348984+00 \\
.1290996+00 \\
.1237184+00 \\
.1187141+00 \\
.1140508+00\end{array}$ & $\begin{array}{l}-.1215251-01 \\
-.1124715-01 \\
-.1043223-01 \\
-.9696623-02 \\
-.9030805-02\end{array}$ & $\begin{array}{l}.2361997-02 \\
.2129103-02 \\
.1924707-02 \\
.1744693-02 \\
.1585628-02\end{array}$ & $\begin{array}{l}.2029262+01 \\
.1965812+01 \\
.1905982+01 \\
.1849471+01 \\
.1796014+01\end{array}$ & $\begin{array}{l}-.2559599+00 \\
-.2428494+00 \\
-.2307946+00 \\
-.2196837+00 \\
-.2094193+00\end{array}$ & $\begin{array}{l}.4057760-01 \\
.3732890-01 \\
.3442294-01 \\
.3181562-01 \\
.2946946-01\end{array}$ & $\begin{array}{l}-.1057033-01 \\
-.9478450-02 \\
-.8525322-02 \\
-.7690260-02 \\
-.6956089-02\end{array}$ & $\begin{array}{l}-.4807378+01 \\
-.4644875+01 \\
-.4492484+01 \\
-.4349310+01 \\
-.4214559+01\end{array}$ & $\begin{array}{l}.8373483+00 \\
.7893267+00 \\
.7454595+00 \\
.7052827+00 \\
.6683947+00\end{array}$ & $\begin{array}{l}-.1866381+00 \\
-.1708462+00 \\
-.1567925+00 \\
-.1442454+00 \\
-.1330092+00\end{array}$ & $\begin{array}{l}.6026066-01 \\
.5379298-01 \\
.4817328-01 \\
.4327174-01 \\
.3898109-01\end{array}$ \\
\hline
\end{tabular}


$(m=12)$

\begin{tabular}{|c|c|c|c|c|c|c|c|c|c|c|c|c|}
\hline $\mathrm{T}^{*}$ & $\mathrm{~B}^{*(0)}$ & $\mathrm{B}^{*(1)}$ & $\mathrm{B}^{*(2)}$ & $\mathrm{B}^{*(3)}$ & $\mathrm{T}^{*} \frac{\partial \mathrm{B}^{*(0)}}{\partial \mathrm{T}^{*}}$ & $\mathrm{~T}^{*} \frac{\partial \mathrm{B}^{*(1)}}{\partial \mathrm{T}^{*}}$ & $\mathrm{~T}^{*} \frac{\partial \mathrm{B}^{*(2)}}{\partial \mathrm{T}^{*}}$ & $\mathrm{~T}^{*} \frac{\partial \mathrm{B}^{*(3)}}{\partial \mathrm{T}^{*}}$ & $\mathrm{~T}^{* 2} \frac{\partial^{2} \mathrm{~B}^{*(0)}}{\partial \mathrm{T}^{* 2}}$ & $\mathrm{~T}^{* 2} \frac{\partial^{2} \mathrm{~B}^{*(1)}}{\partial \mathrm{T}^{* 2}}$ & $\mathrm{~T}^{* 2} \frac{\partial^{2} \mathrm{~B}^{*(2)}}{\partial \mathrm{T}^{* 2}}$ & $\mathrm{~T}^{* 2} \frac{\partial^{2} \mathrm{~B}^{*(3)}}{\partial \mathrm{T}^{* 2}}$ \\
\hline $\begin{array}{l}1.900 \\
1.940 \\
1.980 \\
2.020 \\
2.060\end{array}$ & $\begin{array}{l}-.7141474+00 \\
-.6782872+00 \\
-.6441147+00 \\
-.6115159+00 \\
-.5803871+00\end{array}$ & $\begin{array}{l}.1096969+00 \\
.1056245+00 \\
.1018087+00 \\
.9822746-01 \\
.9486102-01\end{array}$ & $\begin{array}{l}-.8426594-02 \\
-.7876941-02 \\
-.7375747-02 \\
-.6917715-02 \\
-.6498235-02\end{array}$ & $\begin{array}{l}.1444628-02 \\
.1319265-02 \\
.1207486-02 \\
.1107545-02 \\
.1017955-02\end{array}$ & $\begin{array}{l}.1745372+01 \\
.1697330+01 \\
.1651692+01 \\
.1608286+01 \\
.1566951+01\end{array}$ & $\begin{array}{l}-.1999163+00 \\
-.1911000+00 \\
-.1829046+00 \\
-.1752722+00 \\
-.1681514+00\end{array}$ & $\begin{array}{l}.2735246-01 \\
.2543722-01 \\
.2370016-01 \\
.2212095-01 \\
.2068197-01\end{array}$ & $\begin{array}{l}-.6308482-02 \\
-.5735427-02 \\
-.5226818-02 \\
-.4774109-02 \\
-.4370051-02\end{array}$ & $\begin{array}{l}-.4087525+01 \\
-.3967576+01 \\
-.3854146+01 \\
-.3746729+01 \\
-.3644865+01\end{array}$ & $\begin{array}{r}.6344467+00 \\
.6031344+00 \\
.5741918+00 \\
.5473852+00 \\
.5225090+00\end{array}$ & $\begin{array}{l}-.1229176+00 \\
-.1138289+00 \\
-.1056219+00 \\
-.9819238-01 \\
-.9145058-01\end{array}$ & $\begin{array}{l}.3521224-01 \\
.3189088-01 \\
.2895470-01 \\
.2635130-01 \\
.2403636-01\end{array}$ \\
\hline $\begin{array}{l}2.100 \\
2.140 \\
2.180 \\
2.220 \\
2.260\end{array}$ & $\begin{array}{l}-.5506332+00 \\
-.5221672+00 \\
-.4949092+00 \\
-.4687858+00 \\
-.4437295+00\end{array}$ & $\begin{array}{l}.9169174-01 \\
.8870378-01 \\
.8588287-01 \\
.8321618-01 \\
.8069209-01\end{array}$ & $\begin{array}{l}-.6113277-02 \\
-.5759309-02 \\
-.5433227-02 \\
-.5132295-02 \\
-.4854096-02\end{array}$ & $\begin{array}{l}.9374420-03 \\
.8649136-03 \\
.7994278-03 \\
.7401705-03 \\
.6864361-03\end{array}$ & $\begin{array}{l}.1527544+01 \\
.1489934+01 \\
.1454002+01 \\
.1419637+01 \\
.1386740+01\end{array}$ & $\begin{array}{l}-.1614964+00 \\
-.1552668+00 \\
-.1494261+00 \\
-.1439420+00 \\
-.1387853+00\end{array}$ & $\begin{array}{l}.1936790-01 \\
.1816540-01 \\
.1706280-01 \\
.1604986-01 \\
.1511759-01\end{array}$ & $\begin{array}{l}-.4008471-02 \\
-.3684089-02 \\
-.3392379-02 \\
-.3129446-02 \\
-.2891926-02\end{array}$ & $\begin{array}{l}-.3548144+01 \\
-.3456192+01 \\
-.3368670+01 \\
-.3285269+01 \\
-.3205711+01\end{array}$ & $\begin{array}{l}.4993815+00 \\
.4778419+00 \\
.4577472+00 \\
.4389702+00 \\
.4213975+00\end{array}$ & $\begin{array}{l}-.8531878-01 \\
-.7972955-01 \\
-.7462418-01 \\
-.6995139-01 \\
-.6566627-01\end{array}$ & $\begin{array}{l}.2197232-01 \\
.2012717-01 \\
.1847359-01 \\
.1698813-01 \\
.1565063-01\end{array}$ \\
\hline $\begin{array}{l}2.300 \\
2.340 \\
2.380 \\
2.420 \\
2.460\end{array}$ & $\begin{array}{l}-.4196777+00 \\
-.3965730+00 \\
-.3743618+00 \\
-.3529949+00 \\
-.3324263+00\end{array}$ & $\begin{array}{l}.7830009-01 \\
.7603064-01 \\
.7387505-01 \\
.7182543-01 \\
.6987456-01\end{array}$ & $\begin{array}{l}-.4596487-02 \\
-.4357567-02 \\
-.4135640-02 \\
-.3929196-02 \\
-.3736883-02\end{array}$ & $\begin{array}{l}.6376109-03 \\
.5931598-03 \\
.5526152-03 \\
.5155669-03 \\
.4816546-03\end{array}$ & $\begin{array}{l}.1355219+01 \\
.1324990+01 \\
.1295976+01 \\
.1268105+01 \\
.1241310+01\end{array}$ & $\begin{array}{l}-.1339297+00 \\
-.1293518+00 \\
-.1250301+00 \\
-.1209454+00 \\
-.1170801+00\end{array}$ & $\begin{array}{l}.1425805-01 \\
.1346421-01 \\
.1272988-01 \\
.1204952-01 \\
.1141822-01\end{array}$ & $\begin{array}{l}-.2676908-02 \\
-.2481861-02 \\
-.2304585-02 \\
-.2143154-02 \\
-.1995886-02\end{array}$ & $\begin{array}{l}-.3129740+01 \\
-.3057120+01 \\
-.2987639+01 \\
-.2921100+01 \\
-.2857322+01\end{array}$ & $\begin{array}{l}.4049272+00 \\
.3894683+00 \\
.3749388+00 \\
.3612649+00 \\
.3483799+00\end{array}$ & $\begin{array}{l}-.6172938-01 \\
-.5810598-01 \\
-.5476540-01 \\
-.5168051-01 \\
-.4882722-01\end{array}$ & $\begin{array}{l}.1444370-01 \\
.1335228-01 \\
.1236331-01 \\
.1146540-01 \\
.1064864-01\end{array}$ \\
\hline $\begin{array}{l}2.500 \\
2.600 \\
2.700 \\
2.800 \\
2.900\end{array}$ & $\begin{array}{l}-.3126134+00 \\
-.2661336+00 \\
-.2235863+00 \\
-.1845074+00 \\
-.1485023+00\end{array}$ & $\begin{array}{l}.6801585-01 \\
.6373381-01 \\
.5990966-01 \\
.5647691-01 \\
.5338104-01\end{array}$ & $\begin{array}{l}-.3557489-02 \\
-.3158658-02 \\
-.2819816-02 \\
-.2529895-02 \\
-.2280210-02\end{array}$ & $\begin{array}{l}.4505607-03 \\
.3833958-03 \\
.3286025-03 \\
.2835099-03 \\
.2461010-03\end{array}$ & $\begin{array}{l}.1215532+01 \\
.1155169+01 \\
.1100035+01 \\
.1049480+01 \\
.1002957+01\end{array}$ & $\begin{array}{l}-.1134184+00 \\
-.1050628+00 \\
-.9769845-01 \\
-.9117006-01 \\
-.8535177-01\end{array}$ & $\begin{array}{l}.1083159-01 \\
.9535684-02 \\
.8444458-02 \\
.7518625-02 \\
.6727650-02\end{array}$ & $\begin{array}{l}-.1861303-02 \\
-.1572176-02 \\
-.1338101-02 \\
-.1146856-02 \\
-.9892867-03\end{array}$ & $\begin{array}{l}-.2796139+01 \\
-.2653555+01 \\
-.2524164+01 \\
-.2406232+01 \\
-.2298314+01\end{array}$ & $\begin{array}{l}.3362235+00 \\
.3086740+00 \\
.2846236+00 \\
.2634955+00 \\
.2448272+00\end{array}$ & $\begin{array}{l}-.4618411-01 \\
-.4037567-01 \\
-.3551996-01 \\
-.3142845-01 \\
-.2795565-01\end{array}$ & $\begin{array}{l}.9904331-02 \\
.8312807-02 \\
.7032729-02 \\
.5993349-02 \\
.5142016-02\end{array}$ \\
\hline $\begin{array}{l}3.000 \\
3.100 \\
3.200 \\
3.300 \\
3.400\end{array}$ & $\begin{array}{l}-.1152340+00 \\
-.8441244-01 \\
-.5578704-01 \\
-.2913991-01 \\
-.4280856-02\end{array}$ & $\begin{array}{l}.5057690-01 \\
.4802687-01 \\
.4569934-01 \\
.4356762-01 \\
.4160901-01\end{array}$ & $\begin{array}{l}-.2063880-02 \\
-.1875402-02 \\
-.1710340-02 \\
-.1565087-02 \\
-.1436693-02\end{array}$ & $\begin{array}{l}.2148352-03 \\
.1885233-03 \\
.1662384-03 \\
.1472516-03 \\
.1309847-03\end{array}$ & $\begin{array}{l}.9600032+00 \\
.9202229+00 \\
.8832775+00 \\
.8488746+00 \\
.8167606+00\end{array}$ & $\begin{array}{l}-.8014082-01 \\
-.7545263-01 \\
-.7121703-01 \\
-.6737540-01 \\
-.6387846-01\end{array}$ & $\begin{array}{l}.6047548-02 \\
.5459305-02 \\
.4947710-02 \\
.4500494-02 \\
.4107688-02\end{array}$ & $\begin{array}{l}-.8584507-03 \\
-.7490291-03 \\
-.6569042-03 \\
-.5788584-03 \\
-.5123557-03\end{array}$ & $\begin{array}{l}-.2199196+01 \\
-.2107850+01 \\
-.2023402+01 \\
-.1945105+01 \\
-.1872313+01\end{array}$ & $\begin{array}{l}.2282442+00 \\
.2134413+00 \\
.2001670+00 \\
.1882134+00 \\
.1774066+00\end{array}$ & $\begin{array}{l}-.2498813-01 \\
-.2243658-01 \\
-.2022998-01 \\
-.1831146-01 \\
-.1663504-01\end{array}$ & $\begin{array}{l}.4439073-02 \\
.3854313-02 \\
.3364491-02 \\
.2951542-02 \\
.2601304-02\end{array}$ \\
\hline $\begin{array}{l}3.500 \\
3.600 \\
3.700 \\
3.800 \\
3.900\end{array}$ & $\begin{array}{l}.1895689-01 \\
.4072019-01 \\
.6113885-01 \\
.8032801-01 \\
.9839019-01\end{array}$ & $\begin{array}{l}.3980408-01 \\
.3813616-01 \\
.3659084-01 \\
.3515559-01 \\
.3381952-01\end{array}$ & $\begin{array}{l}-.1322723-02 \\
-.1221158-02 \\
-.1130315-02 \\
-.1048780-02 \\
-.9753583-03\end{array}$ & $\begin{array}{l}.1169755-03 \\
.1048517-03 \\
.9431160-04 \\
.8510878-04 \\
.7704094-04\end{array}$ & $\begin{array}{l}.7867145+00 \\
.7585429+00 \\
.7320758+00 \\
.7071630+00 \\
.6836714+00\end{array}$ & $\begin{array}{l}-.6068450-01 \\
-.5775805-01 \\
-.5506877-01 \\
-.5259058-01 \\
-.5030095-01\end{array}$ & $\begin{array}{l}.3761132-02 \\
.3454102-02 \\
.3181023-02 \\
.2937246-02 \\
.2718870-02\end{array}$ & $\begin{array}{l}-.4553811-03 \\
-.4063207-03 \\
-.3638734-03 \\
-.3269826-03 \\
-.2947854-03\end{array}$ & $\begin{array}{l}-.1804467+01 \\
-.1741083+01 \\
-.1681735+01 \\
-.1626052+01 \\
-.1573706+01\end{array}$ & $\begin{array}{l}.1676008+00 \\
.1586727+00 \\
.1505178+00 \\
.1430467+00 \\
.1361826+00\end{array}$ & $\begin{array}{l}-.1516331-01 \\
-.1386563-01 \\
-.1271670-01 \\
-.1169555-01 \\
-.1078467-01\end{array}$ & $\begin{array}{l}.2302583-02 \\
.2046456-02 \\
.1825764-02 \\
.1634719-02 \\
.1468614-02\end{array}$ \\
\hline $\begin{array}{l}4.000 \\
4.100 \\
4.200 \\
4.300 \\
4.400\end{array}$ & $\begin{array}{l}.1154169+00 \\
.1314902+00 \\
.1466837+00 \\
.1610638+00 \\
.1746904+00\end{array}$ & $\begin{array}{l}.3257308-01 \\
.3140788-01 \\
.3031653-01 \\
.2929249-01 \\
.2832993-01\end{array}$ & $\begin{array}{l}-.9090400-03 \\
-.8489628-03 \\
-.7943893-03 \\
-.7446857-03 \\
-.6993052-03\end{array}$ & $\begin{array}{l}.6994101-04 \\
.6367022-04 \\
.5811276-04 \\
.5317145-04 \\
.4876444-04\end{array}$ & $\begin{array}{r}.6614830+00 \\
.6404922+00 \\
.6206045+00 \\
.6017352+00 \\
.5838082+00\end{array}$ & $\begin{array}{l}-.4818032-01 \\
-.4621169-01 \\
-.4438015-01 \\
-.4267264-01 \\
-.4107763-01\end{array}$ & $\begin{array}{l}.2522610-02 \\
.2345679-02 \\
.2185708-02 \\
.2040669-02 \\
.1908824-02\end{array}$ & $\begin{array}{l}-.2665721-03 \\
-.2417567-03 \\
-.2198518-03 \\
-.2004505-03 \\
-.1832116-03\end{array}$ & $\begin{array}{l}-.1524405+01 \\
-.1477892+01 \\
-.1433938+01 \\
-.1392337+01 \\
-.1352906+01\end{array}$ & $\begin{array}{l}.1298595+00 \\
.1240201+00 \\
.1186145+00 \\
.1135995+00 \\
.1089367+00\end{array}$ & $\begin{array}{l}-.9969359-02 \\
-.9237238-02 \\
-.8577802-02 \\
-.7982114-02 \\
-.7442531-02\end{array}$ & $\begin{array}{l}.1323595-02 \\
.1196493-02 \\
.1084680-02 \\
.9859742-03 \\
.8985486-03\end{array}$ \\
\hline $\begin{array}{l}4.500 \\
4.600 \\
4.700 \\
4.800 \\
4.900\end{array}$ & $\begin{array}{l}.1876177+00 \\
.1998951+00 \\
.2115673+00 \\
.2226751+00 \\
.2332558+00\end{array}$ & $\begin{array}{l}.2742369-01 \\
.2656915-01 \\
.2576215-01 \\
.2499897-01 \\
.2427628-01\end{array}$ & $\begin{array}{l}-.6577741-03 \\
-.6196807-03 \\
-.5846659-03 \\
-.5524153-03 \\
-.5226530-03\end{array}$ & $\begin{array}{l}.4482242-04 \\
.4128647-04 \\
.3810635-04 \\
.3523898-04 \\
.3264736-04\end{array}$ & $\begin{array}{l}.5667545+00 \\
.5505118+00 \\
.5350237+00 \\
.5202387+00 \\
.5061101+00\end{array}$ & $\begin{array}{l}-.3958494-01 \\
-.3818555-01 \\
-.3687142-01 \\
-.3563539-01 \\
-.3447106-01\end{array}$ & $\begin{array}{l}.1788673-02 \\
.1678919-02 \\
.1578436-02 \\
.1486241-02 \\
.1401478-02\end{array}$ & $\begin{array}{l}-.1678472-03 \\
-.1541137-03 \\
-.1418040-03 \\
-.1307414-03 \\
-.1207745-03\end{array}$ & $\begin{array}{l}-.1315479+01 \\
-.1279908+01 \\
-.1246058+01 \\
-.1213807+01 \\
-.1183045+01\end{array}$ & $\begin{array}{l}.1045929+00 \\
.1005384+00 \\
.9674705-01 \\
.9319570-01 \\
.8986366-01\end{array}$ & $\begin{array}{l}-.6952493-02 \\
-.6506345-02 \\
-.6099197-02 \\
-.5726803-02 \\
-.5385465-02\end{array}$ & $\begin{array}{l}.8208698-03 \\
.7516440-03 \\
.6897747-03 \\
.6343294-03 \\
.5845118-03\end{array}$ \\
\hline $\begin{array}{l}5.000 \\
5.100 \\
5.200 \\
5.300 \\
5.400\end{array}$ & $\begin{array}{l}.2433435+00 \\
.2529696+00 \\
.2621627+00 \\
.2709493+00 \\
.2793537+00\end{array}$ & $\begin{array}{l}.2359104-01 \\
.2294051-01 \\
.2232221-01 \\
.2173388-01 \\
.2117348-01\end{array}$ & $\begin{array}{l}-.4951361-03 \\
-.4696500-03 \\
-.4460048-03 \\
-.4240317-03 \\
-.4035807-03\end{array}$ & $\begin{array}{l}.3029953-04 \\
.2816786-04 \\
.2622834-04 \\
.2446008-04 \\
.2284480-04\end{array}$ & $\begin{array}{l}.4925951+00 \\
.4796546+00 \\
.4672527+00 \\
.4553567+00 \\
.4439360+00\end{array}$ & $\begin{array}{l}-.3337266-01 \\
-.3233503-01 \\
-.3135351-01 \\
-.3042390-01 \\
-.2954237-01\end{array}$ & $\begin{array}{l}.1323395-02 \\
.1251329-02 \\
.1184699-02 \\
.1122987-02 \\
.1065736-02\end{array}$ & $\begin{array}{l}-.1117732-03 \\
-.1036252-03 \\
-.9623328-04 \\
-.8951323-04 \\
-.8339158-04\end{array}$ & $\begin{array}{l}-.1153670+01 \\
-.1125592+01 \\
-.1098725+01 \\
-.1072994+01 \\
-.1048327+01\end{array}$ & $\begin{array}{l}.8673246-01 \\
.8378560-01 \\
.8100825-01 \\
.7838706-01 \\
.7591002-01\end{array}$ & $\begin{array}{l}-.5071949-02 \\
-.4783425-02 \\
-.4517402-02 \\
-.4271684-02 \\
-.4044330-02\end{array}$ & $\begin{array}{l}.5396394-03 \\
.4991251-03 \\
.4624626-03 \\
.4292132-03 \\
.3989961-03\end{array}$ \\
\hline $\begin{array}{l}5.500 \\
5.600 \\
5.800 \\
6.000 \\
6.200\end{array}$ & $\begin{array}{l}.2873986+00 \\
.2951045+00 \\
.3095754+00 \\
.3229044+00 \\
.3352105+00\end{array}$ & $\begin{array}{l}.2063912-01 \\
.2012910-01 \\
.1917589-01 \\
.1830278-01 \\
.1750037-01\end{array}$ & $\begin{array}{l}-.3845179-03 \\
-.3667233-03 \\
-.3345208-03 \\
-.3062380-03 \\
-.2812770-03\end{array}$ & $\begin{array}{l}.2136651-04 \\
.2001117-04 \\
.1762128-04 \\
.1559243-04 \\
.1385961-04\end{array}$ & $\begin{array}{l}.4329629+00 \\
.4224115+00 \\
.4024803+00 \\
.3839722+00 \\
.3667401+00\end{array}$ & $\begin{array}{l}-.2870548-01 \\
-.2791007-01 \\
-.2643251-01 \\
-.2508962-01 \\
-.2386455-01\end{array}$ & $\begin{array}{l}.1012541-02 \\
.9630377-03 \\
.8738486-03 \\
.7959636-03 \\
.7276010-03\end{array}$ & $\begin{array}{l}-.7780421-04 \\
-.7269496-04 \\
-.6371956-04 \\
-.5613717-04 \\
-.4969117-04\end{array}$ & $\begin{array}{l}-.1024661+01 \\
-.1001934+01 \\
-.9590868+00 \\
-.9193931+00 \\
-.8825170+00\end{array}$ & $\begin{array}{l}.7356625-01 \\
.7134592-01 \\
.6724063-01 \\
.6353189-01 \\
.6016787-01\end{array}$ & \begin{tabular}{|}
$-.3833620-02$ \\
$-.3638027-02$ \\
$-.3286890-02$ \\
$-.2981678-02$ \\
$-.2714965-02$
\end{tabular} & $\begin{array}{l}.3714799-03 \\
.3463746-03 \\
.3024136-03 \\
.2654300-03 \\
.2341137-03\end{array}$ \\
\hline
\end{tabular}


$(m=12)$

\begin{tabular}{|c|c|c|c|c|c|c|c|c|c|c|c|c|}
\hline $\mathrm{T}^{*}$ & $\mathrm{~B}^{*(0)}$ & $\mathrm{B}^{*(1)}$ & $\mathrm{B}^{*(2)}$ & $\mathrm{B}^{*(3)}$ & $\mathrm{T}^{*} \frac{\partial \mathrm{B}^{*(0)}}{\partial \mathrm{T}^{*}}$ & $T^{*} \frac{\partial B^{*(1)}}{\partial T^{*}}$ & $\mathrm{~T}^{*} \frac{\partial \mathrm{B}^{*(2)}}{\partial \mathrm{T}^{*}}$ & $\mathrm{~T}^{*} \frac{\partial \mathrm{B}^{*(3)}}{\partial \mathrm{T}^{*}}$ & $\mathrm{~T}^{* 2} \frac{\partial^{2} \mathrm{~B}^{*(0)}}{\partial \mathrm{T}^{* 2}}$ & $T^{* 2} \frac{\partial^{2} B^{*(1)}}{\partial T^{* 2}}$ & $T^{* 2} \frac{\partial^{2} B^{*(2)}}{\partial T^{* 2}}$ & $\mathrm{~T}^{* 2} \frac{\partial^{2} \mathrm{~B}^{*(3)}}{\partial \mathrm{T}^{* 2}}$ \\
\hline $\begin{array}{l}6.400 \\
6.600 \\
6.800 \\
7.000 \\
7.200\end{array}$ & $\begin{array}{l}.3465971+00 \\
.3571546+00 \\
.3669619+00 \\
.3760885+00 \\
.3845958+00\end{array}$ & $\begin{array}{l}.1676067-01 \\
.1607681-01 \\
.1544289-01 \\
.1485378-01 \\
.1430503-01\end{array}$ & $\begin{array}{l}-.2591483-03 \\
-.2394479-03 \\
-.2218402-03 \\
-.2060451-03 \\
-.1918269-03\end{array}$ & $\begin{array}{l}.1237126-04 \\
.1108614-04 \\
.9971037-05 \\
.8998987-05 \\
.8147967-05\end{array}$ & $\begin{array}{l}.3506568+00 \\
.3356111+00 \\
.3215062+00 \\
.3082566+00 \\
.2957870+00\end{array}$ & $\begin{array}{l}-.2274311-01 \\
-.2171322-01 \\
-.2076456-01 \\
-.1988828-01 \\
-.1907672-01\end{array}$ & $\begin{array}{l}.6673107-03 \\
.6139031-03 \\
.5663964-03 \\
.5239744-03 \\
.4859549-03\end{array}$ & $\begin{array}{l}-.4417907-04 \\
-.3943978-04 \\
-.3534412-04 \\
-.3178772-04 \\
-.2868571-04\end{array}$ & $\begin{array}{l}-.8481685+00 \\
-.8160961+00 \\
-.7860805+90 \\
-.7579298+00 \\
-.7314752+00\end{array}$ & $\begin{array}{l}.5710510-01 \\
.5430689-01 \\
.5174210-01 \\
.4938413-01 \\
.4721016-01\end{array}$ & $\begin{array}{l}-.2480737-02 \\
-.2274084-02 \\
-.2090972-02 \\
-.1928064-02 \\
-.1782579-02\end{array}$ & $\begin{array}{l}.2074358-03 \\
.1845811-03 \\
.1648983-03 \\
.1478638-03 \\
.1330530-03\end{array}$ \\
\hline $\begin{array}{l}7.400 \\
7.600 \\
7.800 \\
8.000 \\
8.200\end{array}$ & $\begin{array}{l}.3925381+00 \\
.3999640+00 \\
.4069164+00 \\
.4134340+00 \\
.4195513+00\end{array}$ & $\begin{array}{l}.1379275-01 \\
.1331351-01 \\
.1286433-01 \\
.1244252-01 \\
.1204573-01\end{array}$ & $\begin{array}{l}-.1789866-03 \\
-.1673553-03 \\
-.1567886-03 \\
-.1471630-03 \\
-.1383721-03\end{array}$ & $\begin{array}{l}.7399870-05 \\
.6739724-05 \\
.6155079-05 \\
.5635528-05 \\
.5172326-05\end{array}$ & $\begin{array}{l}.2840307+00 \\
.2729285+00 \\
.2624274+00 \\
.2524801+00 \\
.2430441+00\end{array}$ & $\begin{array}{l}-.1832325-01 \\
-.1762209-01 \\
-.1696820-01 \\
-.1635712-01 \\
-.1578495-01\end{array}$ & $\begin{array}{l}.4517645-03 \\
.4209184-03 \\
.3930051-03 \\
.3676731-03 \\
.3446214-03\end{array}$ & $\begin{array}{l}-.2596860-04 \\
-.2357917-04 \\
-.2147002-04 \\
-.1960167-04 \\
-.1794108-04\end{array}$ & $\begin{array}{l}-.7065677+00 \\
-.6830753+00 \\
-.6608806+00 \\
-.6398787+00 \\
-.6199759+00\end{array}$ & $\begin{array}{l}.4520051-01 \\
.4333811-01 \\
.4160812-01 \\
.3999758-01 \\
.3849509-01\end{array}$ & $\begin{array}{l}-.1652193-02 \\
-.1534946-02 \\
-.1429180-02 \\
-.1333488-02 \\
-.1246665-02\end{array}$ & $\begin{array}{l}.1201195-03 \\
.1087791-03 \\
.9879723-04 \\
.8997903-04 \\
.8216198-04\end{array}$ \\
\hline $\begin{array}{l}8.400 \\
8.600 \\
8.800 \\
9.000 \\
9.200\end{array}$ & $\begin{array}{l}.4252996+00 \\
.4307069+00 \\
.4357987+00 \\
.4405978+00 \\
.4451254+00\end{array}$ & $\begin{array}{l}.1167186-01 \\
.1131903-01 \\
.1098557-01 \\
.1066996-01 \\
.1037084-01\end{array}$ & $\begin{array}{l}-.1303237-03 \\
-.1229382-03 \\
-.1161460-03 \\
-.1098863-03 \\
-.1041058-03\end{array}$ & $\begin{array}{l}.4758094-05 \\
.4386575-05 \\
.4052442-05 \\
.3751141-05 \\
.3478762-05\end{array}$ & $\begin{array}{l}.2340811+00 \\
.2255566+00 \\
.2174394+00 \\
.2097012+00 \\
.2023161+00\end{array}$ & $\begin{array}{l}-.1524822-01 \\
-.1474387-01 \\
-.1426915-01 \\
-.1382164-01 \\
-.1339912-01\end{array}$ & $\begin{array}{l}.3235909-03 \\
.3043574-03 \\
.2867268-03 \\
.2705298-03 \\
.2556187-03\end{array}$ & $\begin{array}{l}-.1646046-04 \\
-.1513631-04 \\
-.1394871-04 \\
-.1288067-04 \\
-.1191766-04\end{array}$ & $\begin{array}{l}-.6010880+00 \\
-.5831393+00 \\
-.5660614+00 \\
-.5497922+00 \\
-.5342756+00\end{array}$ & $\begin{array}{l}.3709063-01 \\
.3577536-01 \\
.3454142-01 \\
.3338183-01 \\
.3229037-01\end{array}$ & $\begin{array}{l}-.1167678-02 \\
-.1095639-02 \\
-.1029777-02 \\
-.9694263-03 \\
-.9140043-03\end{array}$ & $\begin{array}{l}.7520973-04 \\
.6900744-04 \\
.6345789-04 \\
.5847847-04 \\
.5399867-04\end{array}$ \\
\hline $\begin{array}{r}9.400 \\
9.600 \\
9.800 \\
10.000 \\
10.200\end{array}$ & $\begin{array}{l}.4494002+00 \\
.4534398+00 \\
.4572600+00 \\
.4608753+00 \\
.4642990+00\end{array}$ & $\begin{array}{l}.1008700-01 \\
.9817314-02 \\
.9560785-02 \\
.9316495-02 \\
.9083612-02\end{array}$ & $\begin{array}{l}-.9875775-04 \\
-.9380078-04 \\
-.8919830-04 \\
-.8491788-04 \\
-.8093065-04\end{array}$ & $\begin{array}{l}.3231941-05 \\
.3007767-05 \\
.2803718-05 \\
.2617599-05 \\
.2447495-05\end{array}$ & $\begin{array}{l}.1952606+00 \\
.1885135+00 \\
.1820549+00 \\
.1758670+00 \\
.1699332+00\end{array}$ & $\begin{array}{l}-.1299966-01 \\
-.1262147-01 \\
-.1226296-01 \\
-.1192268-01 \\
-.1159934-01\end{array}$ & $\begin{array}{l}.2418639-03 \\
.2291517-03 \\
.2173815-03 \\
.2064645-03 \\
.1963222-03\end{array}$ & $\begin{array}{l}-.1104721-04 \\
-.1025855-04 \\
-.9542394-05 \\
-.8890667-05 \\
-.8296348-05\end{array}$ & $\begin{array}{l}-.5194605+00 \\
-.5053004+00 \\
-.4917528+00 \\
-.4787786+00 \\
-.4663423+00\end{array}$ & $\begin{array}{l}.3126147-01 \\
.3029014-01 \\
.2937188-01 \\
.2850264-01 \\
.2767877-01\end{array}$ & $\begin{array}{r}-.8630030-03 \\
-.8159770-03 \\
-.7725343-03 \\
-.7323295-03 \\
-.6950568-03\end{array}$ & $\begin{array}{l}.4995812-04 \\
.4630489-04 \\
.4299419-04 \\
.3998725-04 \\
.3725041-04\end{array}$ \\
\hline $\begin{array}{l}10.400 \\
10.600 \\
10.800 \\
11.000 \\
11.400\end{array}$ & $\begin{array}{l}.4675432+00 \\
.4706194+00 \\
.4735378+00 \\
.4763080+00 \\
.4814386+00\end{array}$ & $\begin{array}{l}.8861377-02 \\
.8649093-02 \\
.8446124-02 \\
.8251887-02 \\
.7887498-02\end{array}$ & $\begin{array}{l}-.7721087-04 \\
-.7373554-04 \\
-.7048404-04 \\
-.6743786-04 \\
-.6189648-04\end{array}$ & $\begin{array}{l}.2291730-05 \\
.2148832-05 \\
.2017508-05 \\
.1896614-05 \\
.1682195-05\end{array}$ & $\begin{array}{l}.1642383+00 \\
.1587682+00 \\
.1535101+00 \\
.1484520+00 \\
.1388921+00\end{array}$ & $\begin{array}{l}-.1129174-01 \\
-.1099880-01 \\
-.1071952-01 \\
-.1045302-01 \\
-.9955076-02\end{array}$ & $\begin{array}{l}.1868843-03 \\
.1780887-03 \\
.1698794-03 \\
.1622067-03 \\
.1482962-03\end{array}$ & $\begin{array}{l}-.7753306-05 \\
-.7256174-05 \\
-.6800242-05 \\
-.6381364-05 \\
-.5640552-05\end{array}$ & $\begin{array}{l}-.4544111+00 \\
-.4429547+00 \\
-.4319455+00 \\
-.4213577+00 \\
-.4013531+00\end{array}$ & $\begin{array}{l}.2689696-01 \\
.2615421-01 \\
.2544777-01 \\
.2477516-01 \\
.2352253-01\end{array}$ & $\begin{array}{l}-.6604451-03 \\
-.6282536-03 \\
-.5982672-03 \\
-.5702943-03 \\
-.5197185-03\end{array}$ & $\begin{array}{l}.3475432-04 \\
.3247338-04 \\
.3038513-04 \\
.2846987-04 \\
.2509087-04\end{array}$ \\
\hline $\begin{array}{l}11.800 \\
12.200 \\
12.600 \\
13.000 \\
13.400\end{array}$ & $\begin{array}{l}.4860743+00 \\
.4902696+00 \\
.4940715+00 \\
.4975209+00 \\
.5006536+00\end{array}$ & $\begin{array}{l}.7552116-02 \\
.7242488-02 \\
.6955828-02 \\
.6689728-02 \\
.6442105-02\end{array}$ & $\begin{array}{l}-.5699665-04 \\
-.5264441-04 \\
-.4876220-04 \\
-.4528560-04 \\
-.4216076-04\end{array}$ & $\begin{array}{l}.1498782-05 \\
.1340991-05 \\
.1204518-05 \\
.1085895-05 \\
.9823073-06\end{array}$ & $\begin{array}{l}.1300091+00 \\
.1217343+00 \\
.1140082+00 \\
.1067784+00 \\
.9999925-01\end{array}$ & $\begin{array}{l}-.9499135-02 \\
-.9080259-02 \\
-.8694242-02 \\
-.8337479-02 \\
-.8006865-02\end{array}$ & $\begin{array}{l}.1360500-03 \\
.1252174-03 \\
.1155931-03 \\
.1070069-03 \\
.9931732-04\end{array}$ & $\begin{array}{l}-.5009209-05 \\
-.4467973-05 \\
-.4001430-05 \\
-.3597205-05 \\
-.3245294-05\end{array}$ & $\begin{array}{l}-.3827712+00 \\
-.3654655+00 \\
-.3493090+00 \\
-.3341911+00 \\
-.3200148+00\end{array}$ & $\begin{array}{l}.2238034-01 \\
.2133513-01 \\
.2037551-01 \\
.1949175-01 \\
.1867553-01\end{array}$ & $\begin{array}{l}-.4753510-03 \\
-.4362376-03 \\
-.4015981-03 \\
-.3707896-03 \\
-.3432793-03\end{array}$ & $\begin{array}{l}.2222025-04 \\
.1976675-04 \\
.1765790-04 \\
.1583572-04 \\
.1425353-04\end{array}$ \\
\hline $\begin{array}{l}13.800 \\
14.200 \\
14.600 \\
15.000 \\
16.000\end{array}$ & $\begin{array}{l}.5035008+00 \\
.5060901+00 \\
.5084457+00 \\
.5105892+00 \\
.5151403+00\end{array}$ & $\begin{array}{l}.6211141-02 \\
.5995250-02 \\
.5793034-02 \\
.5603263-02 \\
.5176435-02\end{array}$ & $\begin{array}{l}-.3934237-04 \\
-.3679216-04 \\
-.3447757-04 \\
-.3237079-04 \\
-.2786452-04\end{array}$ & $\begin{array}{l}.8914540-06 \\
.8114432-06 \\
.7407097-06 \\
.6779507-06 \\
.5492089-06\end{array}$ & $\begin{array}{l}.9363051-01 \\
.8763652-01 \\
.8198565-01 \\
.7664973-01 \\
.6452900-01\end{array}$ & $\begin{array}{l}-.7699710-02 \\
-.7413678-02 \\
-.7146723-02 \\
-.6897056-02 \\
-.6338601-02\end{array}$ & $\begin{array}{l}.9240588-04 \\
.8617282-04 \\
.8053367-04 \\
.7541653-04 \\
.6452532-04\end{array}$ & $\begin{array}{l}-.2937549-05 \\
-.2667292-05 \\
-.2429015-05 \\
-.2218148-05 \\
-.1787379-05\end{array}$ & $\begin{array}{l}-.3066950+00 \\
-.2941566+00 \\
-.2823328+00 \\
-.2711645+00 \\
-.2457780+00\end{array}$ & $\begin{array}{l}.1791965-01 \\
.1721790-01 \\
.1656486-01 \\
.1595581-01 \\
.1459962-01\end{array}$ & $\begin{array}{l}-.3186222-03 \\
-.2964450-03 \\
-.2764328-03 \\
-.2583181-03 \\
-.2199176-03\end{array}$ & $\begin{array}{l}.1287334-04 \\
.1166420-04 \\
.1060060-04 \\
.9661421-05 \\
.7749635-05\end{array}$ \\
\hline $\begin{array}{l}17.000 \\
18.000 \\
19.000 \\
20.000 \\
21.000\end{array}$ & $\begin{array}{l}.5187265+00 \\
.5215359+00 \\
.5237139+00 \\
.5253742+00 \\
.5266070+00\end{array}$ & $\begin{array}{l}.4806915-02 \\
.4484104-02 \\
.4199838-02 \\
.3947731-02 \\
.3722721-02\end{array}$ & $\begin{array}{l}-.2422430-04 \\
-.2124370-04 \\
-.1877391-04 \\
-.1670558-04 \\
-.1495693-04\end{array}$ & $\begin{array}{l}.4510865-06 \\
.3750107-06 \\
.3151300-06 \\
.2673591-06 \\
.2287877-06\end{array}$ & $\begin{array}{l}.5389813-01 \\
.4450317-01 \\
.3614466-01 \\
.2866377-01 \\
.2193242-01\end{array}$ & $\begin{array}{l}-.5858676-02 \\
-.5442196-02 \\
-.5077655-02 \\
-.4756135-02 \\
-.4470622-02\end{array}$ & $\begin{array}{l}.5578634-04 \\
.4867489-04 \\
.4281555-04 \\
.3793432-04 \\
.3382759-04\end{array}$ & $\begin{array}{l}-.1460928-05 \\
-.1209142-05 \\
-.1011905-05 \\
-.8552527-06 \\
-.7292875-06\end{array}$ & $\begin{array}{l}-.2234846+00 \\
-.2037538+00 \\
-.1861700+00 \\
-.1704027+00 \\
-.1561863+00\end{array}$ & $\begin{array}{l}.1344112-01 \\
.1244123-01 \\
.1157036-01 \\
.1080573-01 \\
.1012955-01\end{array}$ & $\begin{array}{l}-.1892733-03 \\
-.1644605-03 \\
-.1441105-03 \\
-.1272295-03 \\
-.1130832-03\end{array}$ & $\begin{array}{l}.6307851-05 \\
.5200773-05 \\
.4337094-05 \\
.3653724-05 \\
.3106154-05\end{array}$ \\
\hline $\begin{array}{l}22.000 \\
23.000 \\
24.000 \\
25.000 \\
26.000\end{array}$ & $\begin{array}{l}.5274845+00 \\
.5280651+00 \\
.5283962+00 \\
.5285169+00 \\
.5284595+00\end{array}$ & $\begin{array}{l}.3520744-02 \\
.3338501-02 \\
.3173289-02 \\
.3022872-02 \\
.2885384-02\end{array}$ & $\begin{array}{l}-.1346588-04 \\
-.1218462-04 \\
-.1107588-04 \\
-.1011025-04 \\
-.9264321-05\end{array}$ & $\begin{array}{l}.1973051-06 \\
.1713555-06 \\
.1497748-06 \\
.1316811-06 \\
.1163973-06\end{array}$ & $\begin{array}{r}.1584626-01 \\
.1031932-01 \\
.5280227-02 \\
.6691794-03 \\
-.3564282-02\end{array}$ & $\begin{array}{l}-.4215527-02 \\
-.3986347-02 \\
-.3779415-02 \\
-.3591715-02 \\
-.3420745-02\end{array}$ & $\begin{array}{l}.3034170-04 \\
.2735895-04 \\
.2478805-04 \\
.2255737-04 \\
.2061006-04\end{array}$ & $\begin{array}{l}-.6268658-06 \\
-.5427465-06 \\
-.4730245-06 \\
-.4147523-06 \\
-.3656757-06\end{array}$ & $\begin{array}{l}-.1433044+00 \\
-.1315792+00 \\
-.1208630+00 \\
-.1110324+00 \\
-.1019832+00\end{array}$ & $\begin{array}{l}.9527718-02 \\
.8988941-02 \\
.8504065-02 \\
.8065600-02 \\
.7667359-02\end{array}$ & $\begin{array}{l}-.1011195-03 \\
-.9091788-04 \\
-.8215323-04 \\
-.7457149-04 \\
-.6797180-04\end{array}$ & $\begin{array}{l}.2662381-05 \\
.2299023-05 \\
.1998724-05 \\
.1748417-05 \\
.1538140-05\end{array}$ \\
\hline $\begin{array}{l}27.000 \\
28.000 \\
29.000 \\
30.000 \\
32.000\end{array}$ & $\begin{array}{l}.5282509+00 \\
.5279135+00 \\
.5274664+00 \\
.5269255+00 \\
.5256147+00\end{array}$ & $\begin{array}{l}.2759259-02 \\
.2643169-02 \\
.2535985-02 \\
.2436739-02 \\
.2258837-02\end{array}$ & $\begin{array}{l}-.8519232-05 \\
-.7859686-05 \\
-.7273163-05 \\
-.6749343-05 \\
-.5856936-05\end{array}$ & $\begin{array}{l}.1033982-06 \\
.9227201-07 \\
.8269299-07 \\
.7440099-07 \\
.6088018-07\end{array}$ & $\begin{array}{l}-.7463032-02 \\
-.1106373-01 \\
-.1439792-01 \\
-.1749286-01 \\
-.2305682-01\end{array}$ & $\begin{array}{l}-.3264415-02 \\
-.3120963-02 \\
-.2988898-02 \\
-.2866944-02 \\
-.2649153-02\end{array}$ & $\begin{array}{l}.1890058-04 \\
.1739213-04 \\
.1605469-04 \\
.1486361-04 \\
.1284237-04\end{array}$ & $\begin{array}{l}-.3240526-06 \\
-.2885211-06 \\
-.2580077-06 \\
-.2316570-06 \\
-.1888315-06\end{array}$ & $\begin{array}{l}-.9362699-01 \\
-.8588821-01 \\
-.7870187-01 \\
-.7201179-01 \\
-.5993132-01\end{array}$ & $\begin{array}{l}.7304195-02 \\
.6971788-02 \\
.6666489-02 \\
.6385197-02 \\
.5884391-02\end{array}$ & $\begin{array}{l}-.6219380-04 \\
-.5710829-04 \\
-.5261027-04 \\
-.4861372-04 \\
-.4185321-04\end{array}$ & $\begin{array}{l}.1360228-05 \\
.1208702-05 \\
.1078857-05 \\
.9669516-06 \\
.7855962-06\end{array}$ \\
\hline
\end{tabular}


$(m=12)$

\begin{tabular}{|c|c|c|c|c|c|c|c|c|c|c|c|c|}
\hline $\mathrm{T}^{*}$ & $\mathrm{~B}^{*(0)}$ & $\mathrm{B}^{*(1)}$ & $\mathrm{B}^{*(2)}$ & (3) & $\mathrm{T}^{*} \frac{\partial \mathrm{B}^{*(0)}}{\partial \mathrm{T}^{*}}$ & $\mathrm{~T}^{*} \frac{\partial \mathrm{B}^{*(1)}}{\partial \mathrm{T}^{*}}$ & $\mathrm{~T}^{*} \frac{\partial \mathrm{B}^{*(2)}}{\partial \mathrm{T}^{*}}$ & $T^{*} \frac{\partial B^{*(3)}}{\partial T^{*}}$ & $T^{* 2} \frac{\partial^{2} B^{*(0)}}{\partial T^{* 2}}$ & $T^{* 2} \frac{\partial^{2} B^{*(1)}}{\partial T^{* 2}}$ & $T^{* 2} \frac{\partial^{2} B^{*(2)}}{\partial T^{* 2}}$ & $\mathrm{~T}^{* 2} \frac{\partial^{2} \mathrm{~B}^{*(3)}}{\partial \mathrm{T}^{* 2}}$ \\
\hline & & & & & $186-01$ & & 20233-04 & $559669-06$ & $.4932497-01$ & $.5452243-02$ & $-.3638969-04$ & $.6469184-06$ \\
\hline & & & & & & & & & & $5846-02$ & -.3191465 & $.5390801-06$ \\
\hline & $5062+00$ & $8011-02$ & $.4026492-05$ & Gen & $363-01$ & -.21 & 2782 & $-.1100309-06$ & $-.3159028-01$ & $5298-02$ & $-.2820552-04$ & $.4539702-06$ \\
\hline & $5750+00$ & $+1052-02$ & $.3603197-05$ & $.3060420-07$ & $-.3931154-01$ & $-.2021518-02$ & & & $-.2410914-01$ & 2883 & -.2509860 & $.3859056-06$ \\
\hline 42.000 & & $15257-02$ & $.3242947-05$ & $.2636037-07$ & $-.4231404-01$ & $-.1906476-02$ & $.6991607-05$ & $-.8054852-07$ & $-.1737355-01$ & $2504-02$ & $-.2247142-04$ & $.3308249-06$ \\
\hline 44.000 & 00 & $.1558994-02$ & -.2 & $.2287126-07$ & $-.4501111-01$ & -.18 & $.6308370-05$ & $-.6972160-07$ & $-.1128032-01$ & $.3959281-02$ & $-.2023094-04$ & $.2857742-06$ \\
\hline & & & & & $-.4744390-01$ & & & & & & $-.1830548-04$ & $.2485698-06$ \\
\hline & & & 05 & & & & & & & & 4 & $45-06$ \\
\hline & & $5200-02$ & 5 & .15 & -.51 & & & -.4698371 & $.3927095-02$ & $6530-02$ & $-.1518777-04$ & $.1915443-06$ \\
\hline 52.000 & $7+00$ & $9-02$ & 55 & .137 & $162-01$ & $103-02$ & $.4371994-05$ & $-.4164797-07$ & $.8172074-02$ & $8927-02$ & $-.1391626-04$ & $.1695227-06$ \\
\hline 54.000 & $199+00$ & $.1231303-02$ & -.18 & .12 & -.55 & -.1 & .4 & 07 & .1 & 02 & $-.1279628-04$ & $0-06$ \\
\hline & & & & & & & & & & & & -06 \\
\hline & & & & & & & & & & & & \\
\hline & & & & & 1 & & & & 1 & & & $38213-06$ \\
\hline 64 & +00 & 02 & $1 . x$ & .73 & .61 & 02 & .2 & & -01 & & $379-05$ & $.8920497-07$ \\
\hline 68.000 & $.4905078+00$ & .9459 & 05 & .6 & $-63+2+3$ & - & & 77 & .3 & 02 & $-.7700600-05$ & 11 \\
\hline & & & & & & & & & & & & -07 \\
\hline & & & & & & & & & & & & -07 \\
\hline & & & & & & & & & & & & -07 \\
\hline & & .7 & $.7473338-06$ & .327 & $.6920767-01$ & & $080-05$ & & $.4707068-01$ & $41-02$ & $-.4865747-05$ & .388 \\
\hline 88.000 & 4731 & 03 & -.67836 & .2 & 1 & $.75-30$ & .1 & 08 & & & - . & .337745 \\
\hline & & & & & & & & & & & & \\
\hline & & & & & & & & & & & & -07 \\
\hline & & & & & & & & & & & & -07 \\
\hline & & .5 & 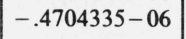 & .16 & .73 & .0. & .97 & 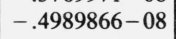 & .58 & .14 & $4401-05$ & .198 \\
\hline 110.00 & .45708 & 48689 & .427372 & $4797>$ & $-.7391740-01$ & _ & & $-.4345579-08$ & 607249 & 1326 & $-.2729967-05$ & 7 \\
\hline & & & & & & & & & & & & \\
\hline & & & & & & & & & & & & -07 \\
\hline & & & & & & & & & & & & \\
\hline & & & 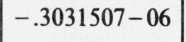 & .90 & -.75 & & $.6214248-06$ & . & $.6699325-01$ & $.1092689-02$ & $-.1916027-05$ & $.1047235-07$ \\
\hline 00 & 44171 & $59207-2 \cdot-3$ & $2805958-0$ & 8124812 & 7605235 & $4887240-03$ & 574790 & 0271094 ( & & & $10<0$ & \\
\hline & & & & & & & & & & & & .0 \\
\hline & & & 24 & & & & & & & & & -08 \\
\hline & & & & .59 & -.7 & & & & & & & \\
\hline & & & & & $-.7706408-01$ & $32-03$ & $.4039175-06$ & $-1450801-00$ & $.7288936-01$ & $.8600368-03$ & $-.1238638-05$ & $.5657030-08$ \\
\hline 170.000 & $40185+0$ & $68231-0 \quad-2$ & $1753301-0$ & $.4155222-09$ & $-.7728163-$ & $-.3760678-0$ & & & & & & \\
\hline & & & & & & & & & & & & .3 \\
\hline & & & & & & & & & & & & \\
\hline & & & & & & & & & & & & \\
\hline & & $.2661412-$ & (2) & $.2255255-$ & 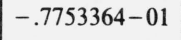 & .2001014 & $.2308886-06$ & $-.6505243-09$ & $.7803181-01$ & $.6301085-03$ & $-.7035667-06$ & $.2542506-08$ \\
\hline 22 & & & & & - & & & & & & & \\
\hline & & & & & & & & $.7-7>3$ & & & - . & $.1721063-08$ \\
\hline & & & & & & & & & & & & $.1362862-08$ \\
\hline & & & $-.0401040-07$ & .98 & -.7 r & & & & & & & $.1098891-08$ \\
\hline & $.3801279+00$ & .1791249-03 & .5574592-07 & $.8093777-10$ & $-.7652453-01$ & $-.1983914-03$ & $.1116887-06$ & $-.2316691-09$ & $.8139661-01$ & $.4204942-03$ & $-.3379267-06$ & $.8993717-09$ \\
\hline
\end{tabular}


$(m=15)$

\begin{tabular}{|c|c|c|c|c|c|c|c|c|c|c|c|c|}
\hline $\mathrm{T}^{*}$ & $\mathrm{~B}^{*(0)}$ & $\mathrm{B}^{*(1)}$ & $\mathrm{B}^{*(2)}$ & $\mathrm{B}^{*(3)}$ & $\mathrm{T}^{*} \frac{\partial \mathrm{B}^{*(\theta)}}{\partial \mathrm{T}^{*}}$ & $\mathrm{~T}^{*} \frac{\partial \mathrm{B}^{*(1)}}{\partial \mathrm{T}^{*}}$ & $\mathrm{~T}^{*} \frac{\partial \mathrm{B}^{*(2)}}{\partial \mathrm{T}^{*}}$ & $\mathrm{~T}^{*} \frac{\partial \mathrm{B}^{*(3)}}{\partial \mathrm{T}^{*}}$ & $\mathrm{~T}^{* 2} \frac{\partial^{2} \mathrm{~B}^{*(0)}}{\partial \mathrm{T}^{* 2}}$ & $\mathrm{~T}^{* 2} \frac{\partial^{2} \mathrm{~B}^{*(1)}}{\partial \mathrm{T}^{* 2}}$ & $\mathrm{~T}^{* 2} \frac{\partial^{2} \mathrm{~B}^{*(2)}}{\partial \mathrm{T}^{* 2}}$ & $\mathrm{~T}^{* 2} \frac{\partial^{2} \mathrm{~B}^{*(3)}}{\partial \mathrm{T}^{* 2}}$ \\
\hline .300 & $83783+02$ & $413+02$ & $7022+02$ & & & $50680+03$ & & & $.3063799+03$ & $828146+03$ & $.2615871+04$ & 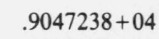 \\
\hline & & $3+01$ & & & $1603+02$ & $.3144757+02$ & $06947+02$ & $.9791091+02$ & $-.9995191+02$ & $.1771844+03$ & $-.3486811+03$ & $.8257908+03$ \\
\hline .500 & $72564+01$ & $7935+01$ & $661179+01$ & $35784+01$ & $55559+02$ & $-.1192669+02$ & $45948+02$ & $-.2007029+02$ & $-.4927675+02$ & $.6050365+02$ & $.8757185+02$ & $.1563294+03$ \\
\hline & $5202771+01$ & $.1897584+01$ & $1096896+01$ & $.1015491+01$ & $.9679453+01$ & $-.5885352+01$ & $.5127201+01$ & $-.6070656+01$ & $-.3002249+02$ & $.2756952+02$ & $-.3122864+02$ & $.4454941+02$ \\
\hline .700 & $-.3922199+01$ & $.1200845+01$ & .54559 & $.4141459+00$ & $.7107760+01$ & & & & $.2069694+02$ & $.1499867+02$ & $-.1385904+02$ & $.1642911+02$ \\
\hline .750 & $.3462400+01$ & $.9907516+00$ & $.4045769+00$ & $19+00$ & $.6245248+01$ & $-.2709892+01$ & $.1730483+01$ & -.1558 & $-.1773303+02$ & $.1159380+02$ & $-.9788922+01$ & $.1069487+02$ \\
\hline .775 & & & & & & & & & & & & 51 \\
\hline .800 & +01 & $9+00$ & $1+00$ & & +01 & $-.2200650+01$ & $1+01$ & +01 & $13757+02$ & $3572+01$ & $991+01$ & $.7222270+01$ \\
\hline .825 & +01 & .7684 & 0 & & $3735+01$ & $-.1997424+01$ & & $86845+00$ & $-.1447765+02$ & $8794+01$ & 146646 & $.6006979+01$ \\
\hline .850 & $-.2762535+01$ & .7114 & .23975 & .14 & $3+01$ & +01 & 0 & +00 & $51861+02$ & $6+01$ & -.5331102 & .50326 \\
\hline .875 & $.2621212+01$ & $.6609698+00$ & $.2131547+00$ & $.1223887+00$ & +01 & $666378+01$ & $615228+00$ & -.645218 & $-.1284626+02$ & $.6724105+01$ & $-.4649845+01$ & $.4244868+01$ \\
\hline .900 & & & & & & $7+01$ & $16243+00$ & +00 & $-.1214886+02$ & $.6113140+01$ & $-.4076941+01$ & $3603064+01$ \\
\hline .925 & 01 & .57 & 00 & 01 & +01 & & & +00 & $59+02$ & $9+01$ & 01 & .307635 \\
\hline .950 & 01 & .5395 & & & & & & & & & & \\
\hline .975 & $18+01$ & $4+00$ & .138 & .698 & +01 & 01 & .5 & +00 & +02 & $6+01$ & -.282628 & .2279 \\
\hline 1.000 & $-.2051947+01$ & $.4773116+00$ & $.1257997+00$ & $.6138796-01$ & .381451 & +01 & +00 & 00 & 01 & 01 & 01 & 976 \\
\hline & & & (1 & & & & & & & & & \\
\hline 40 & 1 & & 0 & & & & & & & & - . & \\
\hline 1.060 & 01 & & & & & & & & & & & .1 \\
\hline & 1 & .39 & 1 & .4174 & +01 & $715+00$ & .3530 & $0+00$ & $3+01$ & $2+01$ & -.179601 & $.1290255+01$ \\
\hline 1.100 & .17 & .38 & -01 & .38125 & $.3282950+01$ & $-.8632752+00$ & $.3275976+00$ & $.1879880+00$ & $-.8358769+01$ & 3212 & $658488+01$ & $16^{7}$ \\
\hline & & & & & & & & & & & & \\
\hline 10 & 01 & .353 & & & & & & & & & & \\
\hline 1.160 & 1 & .340 & & & & & & & & & & $8233+00$ \\
\hline 1.18 & -.14 & & i. & .27 & 01 & 00 & $.2471103+00$ & -00 & $633+01$ & $.2600343+01$ & $-.1228263+01$ & $.7992440+00$ \\
\hline 1.200 & -.14 & .31 & -.63 & .2 & .28756 & $4443+00$ & $.2311997+00$ & $-.1201447+00$ & $-.7188667+01$ & $.2474419+01$ & $-.1144239+01$ & .7308010 \\
\hline & & & & & & & & & & & & \\
\hline & & & 1 & & & & & & & & & \\
\hline 1.2 & 01 & & & & & & & & & & & $9031+00$ \\
\hline & 8. & .2 & $-.5035223-01$ & .1 & & & & & & 01 & - . & $2409+00$ \\
\hline 1.300 & +01 & .266 & 1 & & .2 & 0 & .1 & -.8 & -.6 & .19633 & -.82 & .47 \\
\hline & & & & & & & & & & & & \\
\hline & & & 01 & & & & & & & & & \\
\hline 1. & +01 & & & & & & & & & & & \\
\hline & | & .2357 & $-.3861520-01$ & .12 & -01 & 00 & .1 & -01 &.- & $680+01$ & & +00 \\
\hline 1.400 & $.1050596+01$ & .2290 & -.36 & & +01 & -.4 & +00 & -.5 & -.5 & .159 & -.6 & +00 \\
\hline & & & & & & & & & & & & \\
\hline & & & & & & & & 01 & -.5 & & & +00 \\
\hline 1. & & & & & & & & & & & & \\
\hline & & .2 & $-5032530-01$ & .93 & .21 & 00 & .10 & $.4261792-01$ & $-.5113266+01$ & $.1372379+01$ & $-.4876809+00$ & $.2475087+00$ \\
\hline & & & & & .208 & +00 & $.9868644-01$ & $-.3995892-01$ & $-.5007784+01$ & $.1323934+01$ & $-.4626408+00$ & .231413 \\
\hline & & & & & & & & & 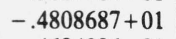 & & & \\
\hline & & & & & & & & & -.4 & & -.3 & $.1787756+00$ \\
\hline & 7. & & & & & & & & & & & \\
\hline & & $.1641200+c$ & $2000035-01$ & .557 & $909+01$ & -.31 & .68 & $-.2473764-01$ & $-.4292305+01$ & $.1015855+01$ & $-.3128510+00$ & $.1403259+00$ \\
\hline & & & & & & & & & & & & .12 \\
\hline & cos & $.1502638+$ & & & & & & 1 & - & & & \\
\hline & $5829248+00$ & $.1440778+$ & 1 & .4095900 & .16 & 0 & .5330389 & 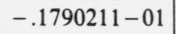 & -.38 & .8518997 & -.2405658 & $.1001671+00$ \\
\hline 1.82 & $.5469657+00$ & $.1383224+00$ & $.1524921-01$ & $.3717666-02$ & & $-.2527107+00$ & $.4931233-01$ & $-.1616848-01$ & -.3748463 & & $-.2215027+00$ & $.9008073-01$ \\
\hline & $-.5128183+00$ & $.1329569+00$ & $-.1421677-01$ & $.3383038-02$ & $.1547806+01$ & $-.2410068+00$ & $.4571768-01$ & $-.1464257-01$ & $-.3632438+01$ & $.7644453+00$ & $-.2044189+00$ & $.8124198-01$ \\
\hline
\end{tabular}


$(m=15)$

\begin{tabular}{|c|c|c|c|c|c|c|c|c|c|c|c|c|}
\hline$T^{*}$ & B & 1) & B & B & $\mathrm{T}^{*} \frac{\partial \mathrm{B}^{*(0)}}{\partial \mathrm{T}^{*}}$ & $\mathrm{~T}^{*} \frac{\partial \mathrm{B}^{*(1)}}{\partial \mathrm{T}^{*}}$ & $\mathrm{~T}^{*} \frac{\partial \mathrm{B}^{*(2)}}{\partial \mathrm{T}^{*}}$ & $\mathrm{~T}^{*} \frac{\partial \mathrm{B}^{*(3)}}{\partial \mathrm{T}^{*}}$ & $T^{* 2} \frac{\partial^{2} B^{*(0)}}{\partial T^{* 2}}$ & $T^{* 2} \frac{\partial^{2} B^{*(1)}}{\partial T^{* 2}}$ & $\mathrm{~T}^{* 2} \frac{\partial^{2} \mathrm{~B}^{*(2)}}{\partial \mathrm{T}^{* 2}}$ & $T^{* 2} \frac{\partial^{2} B^{*(3)}}{\partial T^{* 2}}$ \\
\hline $\begin{array}{l}1.900 \\
1.940 \\
1.980 \\
2.020 \\
2.060\end{array}$ & $\begin{array}{l}-.4803519+00 \\
-.4494478+00 \\
-.4199984+00 \\
-.3919055+00 \\
-.3650796+00\end{array}$ & $\begin{array}{l}.1279453+00 \\
.1232557+00 \\
.1188598+00 \\
.1147325+00 \\
.1108512+00\end{array}$ & $\begin{array}{l}-.1327901-01 \\
-.1242516-01 \\
-.1164591-01 \\
-.1093315-01 \\
-.1027983-01\end{array}$ & $\begin{array}{l}.3086060-02 \\
.2821709-02 \\
.2585730-02 \\
.2374505-02 \\
.2184946-02\end{array}$ & $\begin{array}{l}504155+01 \\
462741+01 \\
423400+01 \\
385979+01 \\
350343+01\end{array}$ & $\begin{array}{l}-.2301672+00 \\
-.2201073+00 \\
-.2107529+00 \\
-.2020381+00 \\
-.1939048+00\end{array}$ & $\begin{array}{l}.4247158-01 \\
.3953256-01 \\
.3686494-01 \\
.3443790-01 \\
.3222473-01\end{array}$ & $\begin{array}{l}-.1329507-01 \\
-.1210138-01 \\
-.1104080-01 \\
-.1009579-01 \\
-.9251465-02\end{array}$ & $\begin{array}{l}-.3523051+01 \\
-.3419760+01 \\
-.3322078+01 \\
-.3229570+01 \\
-.3141841+01\end{array}$ & $\begin{array}{l}.7259012+00 \\
.6903383+00 \\
.6574563+00 \\
.6269918+00 \\
.5987125+00\end{array}$ & $\begin{array}{l}-.1890644+00 \\
-.1752261+00 \\
-.1627216+00 \\
-.1513940+00 \\
-.1411080+00\end{array}$ & $\begin{array}{l}.7346996-01 \\
.6661362-01 \\
.6054627-01 \\
.5516118-01 \\
.5036807-01\end{array}$ \\
\hline $\begin{array}{l}2.100 \\
2.140 \\
2.180 \\
2.220 \\
2.260\end{array}$ & $\begin{array}{l}-.3394389+00 \\
-.3149084+00 \\
-.2914192+00 \\
-.2689082+00 \\
-.2473169+00\end{array}$ & $\begin{array}{l}.1071958+00 \\
.1037483+00 \\
.1004923+00 \\
.9741322-01 \\
.9449776-01\end{array}$ & $\begin{array}{l}-.9679784-02 \\
-.9127586-02 \\
-.8618480-02 \\
-.8148268-02 \\
-.7713235-02\end{array}$ & $\begin{array}{l}.2014409-02 \\
.1860620-02 \\
.1721619-02 \\
.1595708-02 \\
.1481417-02\end{array}$ & $\begin{array}{l}.1316367+01 \\
.1283939+01 \\
.1252955+01 \\
.1223322+01 \\
.1194953+01\end{array}$ & $\begin{array}{l}-.1863012+00 \\
-.1791813+00 \\
-.1725039+00 \\
-.1662322+00 \\
-.1603332+00\end{array}$ & $\begin{array}{l}.3020221-01 \\
.2835008-01 \\
.2665061-01 \\
.2508825-01 \\
.2364932-01\end{array}$ & $\begin{array}{l}-.8495128-02 \\
-.7815922-02 \\
-.7204526-02 \\
-.6652908-02 \\
-.6154131-02\end{array}$ & $\begin{array}{l}-.3058537+01 \\
-.2979337+01 \\
-.2903950+01 \\
-.2832111+01 \\
-.2763578+01\end{array}$ & $\begin{array}{l}.5724133+00 \\
.5479126+00 \\
.5250489+00 \\
.5036786+00 \\
.4836732+00\end{array}$ & $\begin{array}{l}-.1317464+00 \\
-.1232077+00 \\
-.1154031+00 \\
-.1082553+00 \\
-.1016964+00\end{array}$ & $\begin{array}{l}.4609030-01 \\
.4226258-01 \\
.3882904-01 \\
.3574175-01 \\
.3295946-01\end{array}$ \\
\hline $\begin{array}{l}2.300 \\
2.340 \\
2.380 \\
2.420 \\
2.460\end{array}$ & $\begin{array}{l}-.2265917+00 \\
-.2066829+00 \\
-.1875445+00 \\
-.1691339+00 \\
-.1514116+00\end{array}$ & $\begin{array}{l}.9173390-01 \\
.8911074-01 \\
.8661836-01 \\
.8424772-01 \\
.8199057-01\end{array}$ & $-.7310091-02$ & & & $\begin{array}{l}-.1547772+00 \\
-.1495373+00 \\
-.1445893+00 \\
-.1399113+00 \\
-.1354834+00\end{array}$ & & & & $\begin{array}{l}.4649179+00 \\
.4473095+00 \\
.4307555+00 \\
.4151722+00 \\
.4004842+00\end{array}$ & & $\begin{array}{l}.3044654-01 \\
.2817213-01 \\
.2610942-01 \\
.2423505-01 \\
.2252864-01\end{array}$ \\
\hline $\begin{array}{l}2.500 \\
2.600 \\
2.700 \\
2.800 \\
2.900\end{array}$ & $\begin{array}{r}-.1343408+00 \\
-.9429550-01 \\
-.5764079-01 \\
-.2397646-01 \\
.7037441-02\end{array}$ & & & & & & & $\begin{array}{l}-.3983484-02 \\
-.3372157-02 \\
-.2876208-02 \\
-.2470200-02 \\
-.2135039-02\end{array}$ & & & & $\begin{array}{l}.2097233-01 \\
.1763989-01 \\
.1495430-01 \\
.1276953-01 \\
.1097673-01\end{array}$ \\
\hline $\begin{array}{l}3.200 \\
3.300 \\
3.400\end{array}$ & $\begin{array}{l}.8688667-01 \\
.1098316+00 \\
.1312346+00\end{array}$ & $\begin{array}{l}.5961890-01 \\
.5665562-01 \\
.5394921-01 \\
.5146896-01 \\
.4918877-01\end{array}$ & $-.2328934-02$ & & & $\begin{array}{l}-.8283616-01 \\
-.7841485-01 \\
-.7438827-01\end{array}$ & & $\begin{array}{l}-.1856224-02 \\
-.1622623-02 \\
-.1425605-02 \\
-.1258413-02 \\
-.1115715-02\end{array}$ & & & & $\begin{array}{l}.9493780-02 \\
.8258014-02 \\
.7221138-02 \\
.6345564-02 \\
.5601780-02\end{array}$ \\
\hline $\begin{array}{l}3.500 \\
3.600 \\
3.700 \\
3.800 \\
3.900\end{array}$ & $\begin{array}{l}.1875473+00 \\
.2040612+00 \\
.2196033+00\end{array}$ & $\begin{array}{l}.4708629-01 \\
.4514232-01 \\
.4334026-01 \\
.4166568-01 \\
.4010601-01\end{array}$ & $-.1591793-02$ & & & & & & & & & $\begin{array}{l}.4966420-02 \\
.4420838-02 \\
.3950048-02 \\
.3541923-02 \\
.3186583-02\end{array}$ \\
\hline $\begin{array}{l}4.000 \\
4.100 \\
4.200 \\
4.300 \\
4.400\end{array}$ & $\begin{array}{l}.2342526+00 \\
.2480797+00 \\
.2611482+00 \\
.2735153+00 \\
.2852327+00\end{array}$ & $\begin{array}{l}.3601283-01 \\
.3481509-01 \\
.3368876-01\end{array}$ & $\begin{array}{l}-.1301176-02 \\
-.1221173-02 \\
-.1148049-02\end{array}$ & & $\begin{array}{l}.5690815+00 \\
.5509488+00 \\
.5337677+00 \\
.5174651+00 \\
.5019752+00\end{array}$ & $\begin{array}{l}-.5189586-01 \\
-.4992220-01 \\
-.4807789-01\end{array}$ & & & & & & $\begin{array}{l}.2875933-02 \\
.2603301-02 \\
.2363156-02 \\
.2150892-02 \\
.1962656-02\end{array}$ \\
\hline $\begin{array}{l}4.500 \\
4.600 \\
4.700 \\
4.800 \\
4.900\end{array}$ & $\begin{array}{l}.2963472+00 \\
.3069013+00 \\
.3169335+00 \\
.3264791+00 \\
.3355703+00\end{array}$ & $\begin{array}{l}.3162702-01 \\
.3068147-01 \\
.2978690-01 \\
.2893942-01\end{array}$ & $\begin{array}{l}-.9629491-03 \\
-.9107686-03 \\
-.8625673-03\end{array}$ & & $\begin{array}{l}.4732020+00 \\
.4598162+00 \\
.4470371+00 \\
.4348244+00\end{array}$ & \begin{tabular}{|}
$-.4473199-01$ \\
$-.4321084-01$ \\
$-.4177961-01$ \\
$-.4043093-01$
\end{tabular} \mid & & $\begin{array}{l}-.3712909-03 \\
-.3413274-03 \\
-.3144387-03 \\
-.2902460-03 \\
-.2684249-03\end{array}$ & $\begin{array}{l}-.1134254+01 \\
-.1103565+01 \\
-.1074360+01 \\
-.1046533+01 \\
-.1019990+01\end{array}$ & $\begin{array}{l}.1215609+00 \\
.1168952+00 \\
.1125310+00 \\
.1084417+00 \\
.1046038+00\end{array}$ & $\begin{array}{l}-.1108638-01 \\
-.1038508-01 \\
-.9744525-02 \\
-.9158144-02 \\
-.8620213-02\end{array}$ & $\begin{array}{l}.1645796-02 \\
.1512112-02 \\
.1392174-02 \\
.1284289-02\end{array}$ \\
\hline $\begin{array}{l}5.000 \\
5.100 \\
5.200 \\
5.300 \\
5.400\end{array}$ & $\begin{array}{l}.3442364+00 \\
.3525045+00 \\
.3603994+00 \\
.3679438+00 \\
.3751587+00\end{array}$ & $\begin{array}{l}.2813555-01 \\
.2737209-01 \\
.2664618-01 \\
.2595520-01 \\
.2529676-01\end{array}$ & $\begin{array}{l}-.7382041-03 \\
-.7024857-03 \\
-.6692121-03\end{array}$ & $\begin{array}{l}.6843088-04 \\
.6368535-04 \\
.5936295-04 \\
.5541802-04 \\
.5181068-04\end{array}$ & $\begin{array}{l}.4119536+00 \\
.4012309+00 \\
.3909447+00 \\
.3810688+00\end{array}$ & \begin{tabular}{|}
$-.3915822-01$ \\
$-.3795554-01$ \\
$-.3681754-01$ \\
$-.3573938-01$ \\
$-.3471669-01$
\end{tabular} & $\begin{array}{l}.2146362-02 \\
.2031361-02 \\
.1924942-02 \\
.1826297-02 \\
.1734708-02\end{array}$ & $\begin{array}{l}-.2486958-03 \\
-.2308177-03 \\
-.2145814-03 \\
-.1998055-03 \\
-.1863315-03\end{array}$ & $\begin{array}{l}-.9946423+00 \\
-.9704121+00 \\
-.9472267+00 \\
-.9250200+00 \\
-.9037312+00\end{array}$ & $\begin{array}{l}.1009961+00 \\
.9759977-01 \\
.9439788-01 \\
.9137517-01 \\
.8851788-01\end{array}$ & $\begin{array}{l}-.8125721-02 \\
-.7670277-02 \\
-.7250015-02 \\
-.6861526-02 \\
-.6501794-02\end{array}$ & $\begin{array}{l}.1187009-02 \\
.1099083-02 \\
.1019433-02 \\
.9471245-03 \\
.8813445-03\end{array}$ \\
\hline $\begin{array}{l}5.500 \\
5.600 \\
5.800 \\
6.000 \\
6.200\end{array}$ & $\begin{array}{l}.3820637+00 \\
.3886765+00 \\
.4010909+00 \\
.4125210+00 \\
.4230695+00\end{array}$ & $\begin{array}{l}.2466870-01 \\
.2406902-01 \\
.2294767-01 \\
.2191983-01 \\
.2097460-01\end{array}$ & $\begin{array}{l}-.6381701-03 \\
-.6091688-03 \\
-.5566207-03 \\
-.5103940-03 \\
-.4695330-03\end{array}$ & $\begin{array}{l}.4850594-04 \\
.4547303-04 \\
.4011743-04 \\
.3556239-04 \\
.3166496-04\end{array}$ & $\begin{array}{l}.3452135+00 \\
.3292019+00 \\
.3142920+00\end{array}$ & $\begin{array}{l}-.3374548-01 \\
-.3282215-01 \\
-.3110623-01 \\
-.2954583-01 \\
-.2812158-01\end{array}$ & $.1192012-02$ & $\begin{array}{l}-.1740211-03 \\
-.1627531-03 \\
-.1429306-03 \\
-.1261535-03 \\
-.1118651-03\end{array}$ & $\begin{array}{l}-.8833045+00 \\
-.8636885+00 \\
-.8267028+00 \\
-.7924364+00 \\
-.7605997+00\end{array}$ & $\begin{array}{l}.8581359-01 \\
.8325102-01 \\
.7851111-01 \\
.7422686-01 \\
.7033890-01\end{array}$ & $\begin{array}{l}-.6168145-02 \\
-.5858199-02 \\
-.5301168-02 \\
-.4816298-02 \\
-.4392001-02\end{array}$ & $\begin{array}{l}.8213848-03 \\
.7666260-03 \\
.6706057-03 \\
.5896769-03 \\
.5210281-03\end{array}$ \\
\hline
\end{tabular}


$(m=15)$

\begin{tabular}{|c|c|c|c|c|c|c|c|c|c|c|c|c|}
\hline$T^{*}$ & $\mathrm{~B}^{*(0)}$ & 1) & 2) & $\mathrm{B}^{*(3)}$ & $\mathrm{T}^{*} \frac{\partial \mathrm{B}^{*(0)}}{\partial \mathrm{T}^{*}}$ & $\mathrm{~T}^{*} \frac{\partial \mathrm{B}^{*(1)}}{\partial \mathrm{T}^{*}}$ & $\mathrm{~T}^{*} \frac{\partial \mathrm{B}^{*(2)}}{\partial \mathrm{T}^{*}}$ & $\mathrm{~T}^{*} \frac{\partial \mathrm{B}^{*(3)}}{\partial \mathrm{T}^{*}}$ & $T^{* 2} \frac{\partial^{2} B^{*(0)}}{\partial T^{* 2}}$ & $\mathrm{~T}^{* 2} \frac{\partial^{2} \mathrm{~B}^{*(1)}}{\partial \mathrm{T}^{* 2}}$ & $\mathrm{~T}^{* 2} \frac{\partial^{2} \mathrm{~B}^{*(2)}}{\partial \mathrm{T}^{* 2}}$ & $\mathrm{~T}^{* 2} \frac{\partial^{2} \mathrm{~B}^{*(3)}}{\partial \mathrm{T}^{* 2}}$ \\
\hline $\begin{array}{l}6.400 \\
6.600 \\
6.800 \\
7.000 \\
7.200\end{array}$ & $\begin{array}{l}.4328256+00 \\
.4418671+00 \\
.4502622+00 \\
.4580707+00 \\
.4653456+00\end{array}$ & $\begin{array}{l}.2010267-01 \\
.1929606-01 \\
.1854790-01 \\
.1785222-01 \\
.1720382-01\end{array}$ & $\begin{array}{l}.4332534-03 \\
.4009076-03 \\
.3719568-03 \\
.3459503-03 \\
.3225089-03\end{array}$ & $\begin{array}{l}.2831157-04 \\
.2541124-04 \\
.2289054-04 \\
.2068978-04 \\
.1876012-04\end{array}$ & $\begin{array}{l}.3003738+00 \\
.2873516+00 \\
.2751418+00 \\
.2636706+00 \\
.2528731+00\end{array}$ & $\begin{array}{l}-.2681712-01 \\
-.2561854-01 \\
-.2451395-01 \\
-.2349314-01 \\
-.2254730-01\end{array}$ & $\begin{array}{l}.1094867-02 \\
.1008694-02 \\
.9319387-03 \\
.8633092-03 \\
.8017239-03\end{array}$ & $\begin{array}{l}-.9962568-04 \\
-.8908460-04 \\
-.7996028-04 \\
-.7202491-04 \\
-.6509288-04\end{array}$ & $\begin{array}{l}-.7309427+00 \\
-.7032485+00 \\
-.6773283+00 \\
-.6530165+00 \\
-.6301677+00\end{array}$ & $\begin{array}{l}.6679742-01 \\
.6356035-01 \\
.6059198-01 \\
.5786178-01 \\
.5534357-01\end{array}$ & $\begin{array}{l}-.4018879-02 \\
-.3689253-02 \\
-.3396805-02 \\
-.3136299-02 \\
-.2903375-02\end{array}$ & $\begin{array}{l}.4624467-03 \\
.4121772-03 \\
.3688150-03 \\
.3312284-03 \\
.2984988-03\end{array}$ \\
\hline $\begin{array}{l}7.400 \\
7.600 \\
7.800 \\
8.000 \\
8.200\end{array}$ & $\begin{array}{l}.4721339+00 \\
.4784772+00 \\
.4844127+00 \\
.4899737+00 \\
.4951901+00\end{array}$ & $\begin{array}{l}.1659817-01 \\
.1603129-01 \\
.1549966-01 \\
.1500018-01 \\
.1453008-01\end{array}$ & $\begin{array}{l}-.3013118-03 \\
-.2820860-03 \\
-.2645987-03 \\
-.2486496-03 \\
-.2340665-03\end{array}$ & $\begin{array}{l}.1706136-04 \\
.1556019-04 \\
.1422888-04 \\
.1304422-04 \\
.1198669-04\end{array}$ & $\begin{array}{l}.2426917+00 \\
.2330753+00 \\
.2239782+00 \\
.2153595+00 \\
.2071825+00\end{array}$ & $\begin{array}{l}-.2166875-01 \\
-.2085084-01 \\
-.2008773-01 \\
-.1937429-01 \\
-.1870599-01\end{array}$ & $\begin{array}{l}.7462726-03 \\
.6961849-03 \\
.6508061-03 \\
.6095768-03 \\
.5720167-03\end{array}$ & $\begin{array}{l}-.5901203-04 \\
-.5365688-04 \\
-.4892330-04 \\
-.4472450-04 \\
-.4098769-04\end{array}$ & $\begin{array}{l}-.6086533+00 \\
-.5883596+00 \\
-.5691853+00 \\
-.5510402+00 \\
-.5338433+00\end{array}$ & $\begin{array}{l}.5301472-01 \\
.5085566-01 \\
.4884931-01 \\
.4698076-01 \\
.4523693-01\end{array}$ & $\begin{array}{l}-.2694377-02 \\
-.2506224-02 \\
-.2336306-02 \\
-.2182401-02 \\
-.2042612-02\end{array}$ & $\begin{array}{l}.2698758-03 \\
.2447428-03 \\
.2225898-03 \\
.2029929-03 \\
.1855981-03\end{array}$ \\
\hline $\begin{array}{l}8.400 \\
8.600 \\
8.800 \\
9.000 \\
9.200\end{array}$ & $\begin{array}{l}.5000887+00 \\
.5046937+00 \\
.5090270+00 \\
.5131086+00 \\
.5169562+00\end{array}$ & $\begin{array}{l}.1408692-01 \\
.1366849-01 \\
.1327284-01 \\
: 1289820-01 \\
.1254298-01\end{array}$ & $-.2207001-03$ & & $\begin{array}{l}.1994144+00 \\
.1920251+00 \\
.1849879+00 \\
.1782781+00 \\
.1718736+00\end{array}$ & & & $\begin{array}{l}-.3765159-04 \\
-.3466436-04 \\
-.3198193-04 \\
-.2956671-04 \\
-.2738649-04\end{array}$ & $\begin{array}{l}-.5175221+00 \\
-.5020111+00 \\
-.4872515+00 \\
-.4731898+00 \\
-.4597775+00\end{array}$ & $\begin{array}{l}.4360629-01 \\
.4207865-01 \\
.4064497-01 \\
.3929722-01 \\
.3802823-01\end{array}$ & $\begin{array}{l}-.1915307-02 \\
-.1799080-02 \\
-.1692714-02 \\
-.1595151-02 \\
-.1505471-02\end{array}$ & $\begin{array}{l}.1701078-03 \\
.1562713-03 \\
.1438759-03 \\
.1327407-03 \\
.1227112-03\end{array}$ \\
\hline $\begin{array}{r}9.400 \\
9.600 \\
9.800 \\
10.000 \\
10.200\end{array}$ & & & & & & & & $\begin{array}{l}-.2541360-04 \\
-.2362415-04 \\
-.2199746-04 \\
-.2051557-04 \\
-.1916284-04\end{array}$ & & & & $\begin{array}{l}.1136549-03 \\
.1054576-03 \\
.9802094-04 \\
.9125944-04 \\
.8509892-04\end{array}$ \\
\hline $\begin{array}{l}10.400 \\
10.600 \\
10.800 \\
11.000 \\
11.400\end{array}$ & $\begin{array}{l}.5359579+00 \\
.5385571+00 \\
.5410207+00 \\
.5433568+00 \\
.5476769+00\end{array}$ & $\begin{array}{l}.1074775-01 \\
.1049490-01 \\
.1025304-01 \\
.1002150-01 \\
.9586894-02\end{array}$ & $\begin{array}{l}-.1320436-03 \\
-.1262087-03 \\
-.1207449-03 \\
-.1156218-03 \\
-.1062907-03\end{array}$ & & $\begin{array}{l}.1388349+00 \\
.1340862+00 \\
.1295207+00 \\
.1251282+00 \\
.1168246+00\end{array}$ & $\begin{array}{l}-.1344702-01 \\
-.1310336-01 \\
-.1277563-01 \\
-.1246279-01 \\
-.1187799-01\end{array}$ & & $\begin{array}{l}-.1792559-04 \\
-.1679183-04 \\
-.1575104-04 \\
-.1479394-04 \\
-.1309899-04\end{array}$ & & & & $\begin{array}{l}.7947467-04 \\
.7433011-04 \\
.6961561-04 \\
.6528753-04 \\
.5764130-04\end{array}$ \\
\hline $\begin{array}{l}11.800 \\
12.200 \\
12.600 \\
13.000 \\
13.400\end{array}$ & $\begin{array}{l}.5515718+00 \\
.5550884+00 \\
.5582673+00 \\
.5611437+00 \\
.5637483+00\end{array}$ & $\begin{array}{l}.8816754-02 \\
.8474118-02 \\
.8155844-02 \\
.7859475-02\end{array}$ & $\begin{array}{l}-.9067492-04 \\
-.8410734-04 \\
-.7821748-04 \\
-.7291619-04\end{array}$ & $\begin{array}{l}.3532984-05 \\
.3165662-05 \\
.2847475-05 \\
.2570495-05 \\
.2328276-05\end{array}$ & $\begin{array}{l}.1091065+00 \\
.1019148+00 \\
.9519773-01 \\
.8891037-01 \\
.8301313-01\end{array}$ & $\begin{array}{l}-.1134220-01 \\
-.1084967-01 \\
-.1039552-01 \\
-.9975545-02 \\
-.9586144-02\end{array}$ & $\begin{array}{l}.2296434-03 \\
.2116792-03 \\
.1956950-03 \\
.1814146-03 \\
.1686078-03\end{array}$ & $\begin{array}{l}-.1165196-04 \\
-.1040937-04 \\
-.9336523-05 \\
-.8405536-05 \\
-.7593818-05\end{array}$ & $\begin{array}{l}-.3287545+00 \\
-.3137791+00 \\
-.2997959+00 \\
-.2867096+00 \\
-.2744365+00\end{array}$ & $\begin{array}{l}.2648399-01 \\
.2526361-01 \\
.2414259-01 \\
.2310968-01 \\
.2215523-01\end{array}$ & & $\begin{array}{l}.5113391-04 \\
.4556248-04 \\
.4076574-04 \\
.3661449-04 \\
.3300437-04\end{array}$ \\
\hline $\begin{array}{l}13.800 \\
14.200 \\
14.600 \\
15.000 \\
16.000\end{array}$ & $\begin{array}{l}.5661081+00 \\
.5682468+00 \\
.5701853+00 \\
.5719421+00 \\
.5756430+00\end{array}$ & $\begin{array}{l}.7324156-02 \\
.7081683-02 \\
.6854001-02 \\
.6341402-02\end{array}$ & $\begin{array}{l}-.6379052-04 \\
-.5984848-04 \\
-.5625600-04 \\
-.4855652-04\end{array}$ & $\begin{array}{l}.2115345-05 \\
.1927951-05 \\
.1761900-05 \\
.1614388-05 \\
.1311187-05\end{array}$ & $\begin{array}{l}.7747120-01 \\
.7225379-01 \\
.6733355-01 \\
.6268612-01 \\
.5212376-01\end{array}$ & $-.7617708-02$ & & $\begin{array}{l}-.6882947-05 \\
-.6257799-05 \\
-.5705882-05 \\
-.5216819-05 \\
-.4215627-05\end{array}$ & $\begin{array}{l}-.2629032+00 \\
-.2520448+00 \\
-.2418037+00 \\
-.2321288+00 \\
-.2101311+00\end{array}$ & $\begin{array}{l}.2127093-01 \\
.2044958-01 \\
.1968490-01 \\
.1897141-01 \\
.1738149-01\end{array}$ & $\begin{array}{l}-.5345943-03 \\
-.4980268-03 \\
-.4649879-03 \\
-.4350458-03 \\
-.3714467-03\end{array}$ & $\begin{array}{l}.2985055-04 \\
.2708359-04 \\
.2464627-04 \\
.2249121-04 \\
.1809478-04\end{array}$ \\
\hline $\begin{array}{l}17.000 \\
18.000 \\
19.000 \\
20.000 \\
21.000\end{array}$ & $\begin{array}{l}.5785189+00 \\
.5807315+00 \\
.5824058+00 \\
.5836395+00 \\
.5845101+00\end{array}$ & $\begin{array}{l}.5897027-02 \\
.5508327-02 \\
.5165625-02 \\
.4861343-02 \\
.4589468-02\end{array}$ & $\begin{array}{l}-.4231963-04 \\
-.3719971-04 \\
-.3294701-04 \\
-.2937750-04 \\
-.2635326-04\end{array}$ & $\begin{array}{l}.1079468-05 \\
.8993597-06 \\
.7572629-06 \\
.6436573-06 \\
.5517443-06\end{array}$ & $\begin{array}{l}.4285262-01 \\
.3465303-01 \\
.2735239-01 \\
.2081324-01 \\
.1492472-01\end{array}$ & $-.5404692-02$ & & $\begin{array}{l}-.3454672-05 \\
-.2866170-05 \\
-.2404006-05 \\
-.2036076-05 \\
-.1739569-05\end{array}$ & $\begin{array}{l}-.1908062+00 \\
-.1736960+00 \\
-.1584417+00 \\
-.1447580+00 \\
-.1324154+00\end{array}$ & $\begin{array}{l}.1602194-01 \\
.1484738-01 \\
.1382343-01 \\
.1292359-01 \\
.1212716-01\end{array}$ & $\begin{array}{l}-.3205532-03 \\
-.2792375-03 \\
-.2452697-03 \\
-.2170271-03 \\
-.1933076-03\end{array}$ & $\begin{array}{l}.1476914-04 \\
.1220833-04 \\
.1020528-04 \\
.8616503-05 \\
.7340499-05\end{array}$ \\
\hline $\begin{array}{l}22.000 \\
23.000 \\
24.000 \\
25.000 \\
26.000\end{array}$ & $\begin{array}{l}.5850794+00 \\
.5853974+00 \\
.5855050+00 \\
.5854355+00 \\
.5852165+00\end{array}$ & $\begin{array}{l}.4345166-02 \\
.4124513-02 \\
.3924288-02 \\
.3741825-02 \\
.3574897-02\end{array}$ & $\begin{array}{l}-.2376933-04 \\
-.2154471-04 \\
-.1961615-04 \\
-.1793363-04 \\
-.1645725-04\end{array}$ & $\begin{array}{l}.4765821-06 \\
.4145198-06 \\
.3628213-06 \\
.3194082-06 \\
.2826832-06\end{array}$ & $\begin{array}{r}.9596441-02 \\
.4753970-02 \\
.3354561-03 \\
-.3710919-02 \\
-.7428895-02\end{array}$ & $\begin{array}{l}-.5101521-02 \\
-.4828911-02 \\
-.4582556-02 \\
-.4358915-02 \\
-.4155047-02\end{array}$ & $\begin{array}{l}.5263335-04 \\
.4754732-04 \\
.4315532-04 \\
.3933768-04 \\
.3599928-04\end{array}$ & $\begin{array}{l}-.1497984-05 \\
-.1299183-05 \\
-.1134105-05 \\
-.9958979-06 \\
-.8793098-06\end{array}$ & $\begin{array}{l}-.1212270+00 \\
-.1110391+00 \\
-.1017244+00 \\
-.9317601-01 \\
-.8530401-01\end{array}$ & $\begin{array}{l}.1141773-01 \\
.1078211-01 \\
.1020966-01 \\
.9691609-02 \\
.9220754-02\end{array}$ & $\begin{array}{l}-.1732059-03 \\
-.1560308-03 \\
-.1412469-03 \\
-.1284350-03 \\
-.1172633-03\end{array}$ & $\begin{array}{l}.6304136-05 \\
.5453825-05 \\
.4749707-05 \\
.4161725-05 \\
.3666926-05\end{array}$ \\
\hline $\begin{array}{l}27.000 \\
28.000 \\
29.000 \\
30.000 \\
32.000\end{array}$ & $\begin{array}{l}.5848710+00 \\
.5844182+00 \\
.5838743+00 \\
.5832528+00 \\
.5818221+00\end{array}$ & $\begin{array}{l}.3421634-02 \\
.3280448-02 \\
.3149990-02 \\
.3029100-02 \\
.2812163-02\end{array}$ & $\begin{array}{l}-.1515483-04 \\
-.1400022-04 \\
-.1297198-04 \\
-.1205240-04 \\
-.1048275-04\end{array}$ & $\begin{array}{l}.2514046-06 \\
.2245975-06 \\
.2014892-06 \\
.1814620-06 \\
.1487529-06\end{array}$ & $\begin{array}{l}-.1085565-01 \\
-.1402297-01 \\
-.1695821-01 \\
-.1968505-01 \\
-.2459317-01\end{array}$ & $\begin{array}{l}-.3968495-02 \\
-.3797185-02 \\
-.3639360-02 \\
-.3493518-02 \\
-.3232809-02\end{array}$ & $\begin{array}{l}.3306381-04 \\
.3046946-04 \\
.2816576-04 \\
.2611118-04 \\
.2261751-04\end{array}$ & $\begin{array}{l}-.7802743-06 \\
-.6956085-06 \\
-.6227971-06 \\
-.5598351-06 \\
-.4573188-06\end{array}$ & $\begin{array}{l}-.7803197-01 \\
-.7129455-01 \\
-.6503558-01 \\
-.5920649-01 \\
-.4867442-01\end{array}$ & $\begin{array}{l}.8791074-02 \\
.8397522-02 \\
.8035829-02 \\
.7702367-02 \\
.7108145-02\end{array}$ & $\begin{array}{l}-.1074663-03 \\
-.9882958-04 \\
-.9117886-04 \\
-.8437103-04 \\
-.7283108-04\end{array}$ & $\begin{array}{l}.3247586-05 \\
.2889868-05 \\
.2582867-05 \\
.2317912-05 \\
.1887658-05\end{array}$ \\
\hline
\end{tabular}


$(m=15)$

\begin{tabular}{|c|c|c|c|c|c|c|c|c|c|c|c|c|}
\hline $\mathrm{T}^{*}$ & $\mathrm{~B}^{*(0)}$ & $\mathrm{B}^{*(1)}$ & $\mathrm{B}^{*(2)}$ & $\mathrm{B}^{*(3)}$ & $\mathrm{T}^{*} \frac{\partial \mathrm{B} *(0)}{\partial \mathrm{T}^{*}}$ & $\mathrm{~T}^{*} \frac{\partial \mathrm{B}^{*(1)}}{\partial \mathrm{T}^{*}}$ & $\mathrm{~T}^{*} \frac{\partial \mathrm{B}^{*(2)}}{\partial \mathrm{T}^{*}}$ & $\mathrm{~T}^{*} \frac{\partial \mathrm{B}^{*(3)}}{\partial \mathrm{T}^{*}}$ & $\mathrm{~T}^{* 2} \frac{\partial^{2} \mathrm{~B}^{*(0)}}{\partial \mathrm{T}^{* 2}}$ & $\mathrm{~T}^{* 2} \frac{\partial^{2} \mathrm{~B}^{*(1)}}{\partial \mathrm{T}^{* 2}}$ & $\mathrm{~T}^{* 2} \frac{\partial^{2} \mathrm{~B}^{*(2)}}{\partial \mathrm{T}^{* 2}}$ & $\mathrm{~T}^{* 2} \frac{\partial^{2} \mathrm{~B}^{*(3)}}{\partial \mathrm{T}^{* 2}}$ \\
\hline J & $1996+00$ & 02 & 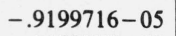 & & 1 & 2 & 4 & 06 & 1 & $790-02$ & 8018-04 & (4) \\
\hline & & & & -06 & 26597 & 02 & 1332 & $-.3168103-06$ & $-.3122764-01$ & $47166-02$ & $-.5580197-04$ & $.1300729-05$ \\
\hline 38.000 & & & -.7249 & & $600608-01$ & & $48092-04$ & $-.2679088-06$ & $-.2392739-01$ & & $-.4942300-04$ & $.1097386-05$ \\
\hline & & $9-02$ & -.6497 & $.7520139-07$ & $.3898815-01$ & $-.2479356-02$ & $.1383699-04$ & $-.2286206-06$ & $-.1738380-01$ & $.5405182-02$ & $-.4406796-04$ & $.9344408-06$ \\
\hline 42.000 & $.5726901+00$ & $.2061224-02$ & -.58575 & $.6484574-07$ & $-.4165924-01$ & $-.2340848-02$ & $.1244009-04$ & $-.1966910-06$ & $-.1148744-01$ & $.5094589-02$ & $-.3953039-04$ & $.8023322-06$ \\
\hline 44.000 & $06957+00$ & $55251-02$ & - & .5632032 & 440628 & -.221 & .112433 & 17040 & $.6148966-02$ & $.4816131-02$ & $-.3565310-04$ & 6940910 \\
\hline & & & & & & & & & & & & \\
\hline 4 & 00 & 02 & & & & & & & & & $-.2942055-04$ & som t \\
\hline & & -02 & & & & & & & & & & \\
\hline 52.000 & +00 & $3-02$ & -.373 & .3403 & -.51635 & $.1823717-02$ & $.7838018-05$ & $.1022694-06$ & .1092824-01 & $.3942326-02$ & $-.2467978-04$ & .4137913 \\
\hline 54.00 & 0 & 1551 & 5 & 07 & 1 & 22 & 05 & 07 & 01 & 02 & 04 & 368 \\
\hline & & & & & & & & & & & & \\
\hline & & & & & & & & & 01 & & $17-04$ & .2958261 \\
\hline 60 & & & & & & & & & & & & \\
\hline 6 & .55 & & 05 & .182 & -01 & 02 & .503020 & 07 & -01 & .308 & -.157136 & .21894 \\
\hline 68.00 & $.5475216+00$ & 98288 & 2130 & .152598 & $6082465-01$ & .133827 & 4423 & .453 & .324 & & -.137 & .18 \\
\hline & & & & & & & & & & & & \\
\hline & & & & & & & & & & & & \\
\hline 80 & & & & & & & & & & & & \\
\hline & & & 5 & -08 & -01 & 2 & & 07 & -01 & .22 & $.8774284-05$ & 96029 \\
\hline 8 & & & & .7 & 1 & 3 & .25 & 7 & & & - . & \\
\hline & & & & & & & & & & & & \\
\hline & & & & & & & & & & & 05 & \\
\hline & & & & & & & & & & & & \\
\hline & & & 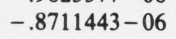 & & & & & & & & 5 & .4 \\
\hline & & & & & & & & & & & & \\
\hline & & & & & & & & & & & & \\
\hline & & & & & & & & & & & & \\
\hline & .5 & & & & & & & & & & & \\
\hline & & & & -0 & -01 & $-.6428474-03$ & $95-05$ & & 1 & $2079-02$ & $-.3501070-05$ & .26 \\
\hline 13 & & & & & 1 & 3 & & & & & & \\
\hline & & & & & & & & & & & & \\
\hline & & & & & & & & & & & & \\
\hline & & & & & & & & & & & & \\
\hline & & & $-.3704000-06$ & $.1236442-08$ & $-.7367782-01$ & $-.5099360-03$ & $.7477009-06$ & $-.3591646-08$ & $436-01$ & $.1077154-02$ & $-.2275994-05$ & $.1410229-07$ \\
\hline 170.0 & & & & & & -4 & & & & & & \\
\hline & & & & & & & & & & & & \\
\hline & & & & & 1 & & & & 01 & & 05 & -08 \\
\hline & .472 & & -.23 & & & & & & & & & \\
\hline & $.4684016+c$ & .345 & 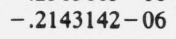 & .56 & $6-01$ & $-.3772165-03$ & .4298689 & $914-08$ & $013-01$ & .7940744 & $-.1301413-$ & .6349410 \\
\hline & & & & & & & & & & & & \\
\hline & & & & & & & & & & & & \\
\hline & & & & & 1 & & & & & & 6 & .34 \\
\hline & .4470 & & 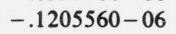 & & 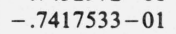 & 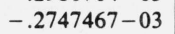 & & & & & & \\
\hline 300.000 & $.4418917+00$ & $.2344937-03$ & $1050680-06$ & $.2013357-09$ & $7398359-01$ & $2547017-03$ & $.2092773-06$ & $.5782263-09$ & $.7708123-01$ & $.5342020-03$ & $-.6298095-06$ & $.2247538-08$ \\
\hline
\end{tabular}


$(m=18)$

\begin{tabular}{|c|c|c|c|c|c|c|c|c|c|c|c|c|}
\hline$T^{*}$ & $\mathrm{~B}^{*(0)}$ & $\mathrm{B}^{*(1)}$ & $\mathrm{B}^{*(2)}$ & $\mathrm{B}^{*(3)}$ & $\Gamma^{*} \frac{\partial B^{*(0)}}{\partial T^{*}}$ & $\mathrm{~T}^{*} \frac{\partial \mathrm{B}^{*(1)}}{\partial \mathrm{T}^{*}}$ & $\mathrm{~T}^{*} \frac{\partial \mathrm{B}^{*(2)}}{\partial \mathrm{T}^{*}}$ & $\mathrm{~T}^{*} \frac{\partial \mathbf{B}^{*(3)}}{\partial \mathrm{T}^{*}}$ & $T^{* 2} \frac{\partial^{2} B^{*(0)}}{\partial T^{* 2}}$ & $T^{* 2} \frac{\partial^{2} B^{*(1)}}{\partial T^{* 2}}$ & $T^{* 2} \frac{\partial^{2} B^{*(2)}}{\partial T^{* 2}}$ & $T^{* 2} \frac{\partial^{2} B^{*(3)}}{\partial T^{* 2}}$ \\
\hline $\begin{array}{l}.300 \\
.400 \\
.500 \\
.600 \\
.700\end{array}$ & $\begin{array}{l}-.2120540+02 \\
-.1041668+02 \\
-.6514690+01 \\
-.4572671+01 \\
-.3425153+01\end{array}$ & $\begin{array}{l}.3071437+02 \\
.8725622+01 \\
.3827153+01 \\
.2114170+01 \\
.1342277+01\end{array}$ & $\begin{array}{l}-.6855396+02 \\
-.1213756+02 \\
-.3723993+01 \\
-.1544986+01 \\
-.7729564+00\end{array}$ & $\begin{array}{l}.2092694+03 \\
.2470386+02 \\
.5608167+01 \\
.1833868+01 \\
.7542897+00\end{array}$ & $\begin{array}{l}.5861919+02 \\
.2322975+02 \\
.1301943+02 \\
.8669428+01 \\
.6372644+01\end{array}$ & $\begin{array}{l}-.1480976+03 \\
-.3459806+02 \\
-.1316194+02 \\
-.6512882+01 \\
-.3784566+01\end{array}$ & $\begin{array}{l}.4449520+03 \\
.6778122+02 \\
.1870643+02 \\
.7164832+01 \\
.3365309+01\end{array}$ & $\begin{array}{l}-.1660300+04 \\
-.1719307+03 \\
-.3560218+02 \\
-.1086331+02 \\
-.4230798+01\end{array}$ & $\begin{array}{l}-.2724983+03 \\
-.8906154+02 \\
-.4397747+02 \\
-.2682924+02 \\
-.1851574+02\end{array}$ & $\begin{array}{l}.9664111+03 \\
.1944879+03 \\
.6657806+02 \\
.3040683+02 \\
.1657687+02\end{array}$ & $\begin{array}{l}-.3577858+04 \\
-.4798693+03 \\
-.1211715+03 \\
-.4341814+02 \\
-.1935217+02\end{array}$ & $\begin{array}{l}.1563089+05 \\
.1442535+04 \\
.2756394+03 \\
.7918637+02 \\
.2941188+02\end{array}$ \\
\hline $\begin{array}{l}.750 \\
.775 \\
.800 \\
.825 \\
.850\end{array}$ & $\begin{array}{l}-.3012822+01 \\
-.2834522+01 \\
-.2671639+01 \\
-.2522279+01 \\
-.2384845+01\end{array}$ & $\begin{array}{l}.1109077+01 \\
.1015455+01 \\
.9337243+00 \\
.8619553+00 \\
.7985916+00\end{array}$ & $\begin{array}{l}-.5747157+00 \\
-.5005892+00 \\
-.4386544+00 \\
-.3865246+00 \\
-.3423478+00\end{array}$ & $\begin{array}{l}.5145510+00 \\
.4303456+00 \\
.3626293+00 \\
.3076968+00 \\
.2627730+00\end{array}$ & $\begin{array}{l}.5601747+01 \\
.5277698+01 \\
.4986662+01 \\
.4724028+01 \\
.4485982+01\end{array}$ & $\begin{array}{l}-.3009732+01 \\
-.2706879+01 \\
-.2446802+01 \\
-.2222006+01 \\
-.2026533+01\end{array}$ & $\begin{array}{l}.2435596+01 \\
.2094985+01 \\
.1813897+01 \\
.1580098+01 \\
.1384206+01\end{array}$ & $\begin{array}{l}-.2819597+01 \\
-.2332740+01 \\
-.1945422+01 \\
-.1634452+01 \\
-.1382644+01\end{array}$ & $\begin{array}{l}-.1587168+02 \\
-.1478533+02 \\
-.1382311+02 \\
-.1296618+02 \\
-.1219917+02\end{array}$ & $\begin{array}{l}.1282627+02 \\
.1138891+02 \\
.1016933+02 \\
.9127272+01 \\
.8231111+01\end{array}$ & $\begin{array}{l}-.1369641+02 \\
-.1165930+02 \\
-.9995371+01 \\
-.8624903+01 \\
-.7487397+01\end{array}$ & $\begin{array}{l}.1920921+02 \\
.1574377+02 \\
.1301251+02 \\
.1083915+02 \\
.9094286+01\end{array}$ \\
\hline $\begin{array}{l}.875 \\
.900 \\
.925 \\
.950 \\
.975\end{array}$ & $\begin{array}{l}-.2257980+01 \\
-.2140525+01 \\
-.2031481+01 \\
-.1929985+01 \\
-.1835290+01\end{array}$ & $\begin{array}{l}.7423671+00 \\
.6922438+00 \\
.6473650+00 \\
.6070183+00 \\
.5706079+00\end{array}$ & $\begin{array}{l}-.3046732+00 \\
-.2723536+00 \\
-.2444749+00 \\
-.2203027+00 \\
-.1992429+00\end{array}$ & $\begin{array}{l}.2257569+00 \\
.1950417+00 \\
.1693873+00 \\
.1478279+00 \\
.1296051+00\end{array}$ & $\begin{array}{l}.4269344+01 \\
.4071447+01 \\
.3890037+01 \\
.3723200+01 \\
.3569299+01\end{array}$ & $\begin{array}{l}-.1855607+01 \\
-.1705367+01 \\
-.1572670+01 \\
-.1454936+01 \\
-.1350035+01\end{array}$ & $\begin{array}{l}.1218959+01 \\
.1078679+01 \\
.9588872+00 \\
.8560261+00 \\
.7672441+00\end{array}$ & $\begin{array}{l}-.1177119+01 \\
-.1008127+01 \\
-.8682098+00 \\
-.7516137+00 \\
-.6538601+00\end{array}$ & $\begin{array}{l}-.1150945+02 \\
-.1088654+02 \\
-.1032172+02 \\
-.9807651+01 \\
-.9338143+01\end{array}$ & $\begin{array}{l}.7455773+01 \\
.6781207+01 \\
.6191245+01 \\
.5672756+01 \\
.5214996+01\end{array}$ & & $\begin{array}{l}.7681778+01 \\
.6529460+01 \\
.5582597+01 \\
.4799283+01 \\
.4147147+01\end{array}$ \\
\hline $\begin{array}{l}1.000 \\
1.020 \\
1.040 \\
1.060 \\
1.080\end{array}$ & $\begin{array}{l}-.1746741+01 \\
-.1679939+01 \\
-.1616442+01 \\
-.1556012+01 \\
-.1498436+01\end{array}$ & $\begin{array}{l}.5376325+00 \\
.5134382+00 \\
.4909824+00 \\
.4700998+00 \\
.4506445+00\end{array}$ & $\begin{array}{l}-.1808113+00 \\
-.1676893+00 \\
-.1558234+00 \\
-.1450670+00 \\
-.1352932+00\end{array}$ & & & & & & $\begin{array}{l}-.8907933+01 \\
-.8589017+01 \\
-.8290362+01 \\
-.8010189+01 \\
-.7746916+01\end{array}$ & $\begin{array}{l}.4809118+01 \\
.4516801+01 \\
.4249795+01 \\
.4005339+01 \\
.3781033+01\end{array}$ & $\begin{array}{l}-.3560962+01 \\
-.3262240+01 \\
-.2995438+01 \\
-.2756476+01 \\
-.2541878+01\end{array}$ & $\begin{array}{l}.3600979+01 \\
.3226894+01 \\
.2899704+01 \\
.2612600+01 \\
.2359886+01\end{array}$ \\
\hline $\begin{array}{l}1.100 \\
1.120 \\
1.140 \\
1.160 \\
1.180\end{array}$ & $\begin{array}{l}-.1443518+01 \\
-.1391080+01 \\
-.1340960+01 \\
-.1293010+01 \\
-.1247093+01\end{array}$ & $\begin{array}{l}.4324870+00 \\
.4155123+00 \\
.3996178+00 \\
.3847119+00 \\
.3707125+00\end{array}$ & $\begin{array}{l}-.1263924+00 \\
-.1182690+00 \\
-.1108398+00 \\
-.1040320+00 \\
-.9778181-01\end{array}$ & $\begin{array}{l}.7127388-01 \\
.6529868-01 \\
.5994291-01 \\
.5513091-01 \\
.5079761-01\end{array}$ & & & & & & & & $\begin{array}{l}.2136783+01 \\
.1939258+01 \\
.1763903+01 \\
.1607821+01 \\
.1468545+01\end{array}$ \\
\hline $\begin{array}{l}1.200 \\
1.220 \\
1.240 \\
1.260 \\
1.280\end{array}$ & $\begin{array}{l}-.1203084+01 \\
-.1160868+01 \\
-.1120339+01 \\
-.1081399+01 \\
-.1043958+01\end{array}$ & $\begin{array}{l}.3575458+00 \\
.3451456+00 \\
.3334519+00 \\
.3224108+00 \\
.3119732+00\end{array}$ & $\begin{array}{l}-.9203300-01 \\
-.8673610-01 \\
-.8184736-01 \\
-.7732801-01 \\
-.7314359-01\end{array}$ & & & $\begin{array}{l}-.7663783+00 \\
-.7342868+00 \\
-.7042480+00 \\
-.6760911+00 \\
-.6496625+00\end{array}$ & $\begin{array}{l}.3309065+00 \\
.3102437+00 \\
.2912740+00 \\
.2738282+00 \\
.2577570+00\end{array}$ & $\begin{array}{l}-.2229802+00 \\
-.2052395+00 \\
-.1892356+00 \\
-.1747687+00 \\
-.1616652+00\end{array}$ & & $\begin{array}{l}.2757152+01 \\
.2627430+01 \\
.2506643+01 \\
.2394006+01 \\
.2288814+01\end{array}$ & & $\begin{array}{l}.1343966+01 \\
.1232274+01 \\
.1131912+01 \\
.1041537+01 \\
.9599880+00\end{array}$ \\
\hline $\begin{array}{l}1.300 \\
1.320 \\
1.340 \\
1.360 \\
1.380\end{array}$ & $\begin{array}{l}-.1007932+01 \\
-.9732430+00 \\
-.9398193+00 \\
-.9075938+00 \\
-.8765040+00\end{array}$ & $\begin{array}{l}.3020947+00 \\
.2927350+00 \\
.2838573+00 \\
.2754279+00 \\
.2674164+00\end{array}$ & $\begin{array}{l}-.6926346-01 \\
-.6566027-01 \\
-.6230956-01 \\
-.5918943-01 \\
-.5628022-01\end{array}$ & $\begin{array}{l}.3217326-01 \\
.2997020-01 \\
.2795548-01 \\
.2610999-01 \\
.2441685-01\end{array}$ & & $\begin{array}{l}-.5794285+00 \\
-.5586566+00 \\
-.5390413+00\end{array}$ & & $\begin{array}{l}-.1497741+00 \\
-.1389632+00 \\
-.1291169+00 \\
-.1201338+00 \\
-.1119245+00\end{array}$ & $\begin{array}{l}-.5648036+01 \\
-.5509256+01 \\
-.5376727+01 \\
-.5250052+01 \\
-.5128864+01\end{array}$ & $\begin{array}{l}.2190439+01 \\
.2098313+01 \\
.2011927+01 \\
.1930821+01 \\
.1854580+01\end{array}$ & $\begin{array}{l}-.1169033+01 \\
-.1098900+01 \\
-.1034243+01 \\
-.9745426+00 \\
-.9193376+00\end{array}$ & $\begin{array}{l}.8862551+00 \\
.8194613+00 \\
.7588413+00 \\
.7037258+00 \\
.6535283+00\end{array}$ \\
\hline $\begin{array}{l}1.400 \\
1.420 \\
1.440 \\
1.460 \\
1.480\end{array}$ & $\begin{array}{l}-.8464919+00 \\
-.8175030+00 \\
-.7894868+00 \\
-.7623958+00 \\
-.7361855+00\end{array}$ & $\begin{array}{l}.2597946+00 \\
.2525368+00 \\
.2456195+00 \\
.2390208+00 \\
.2327209+00\end{array}$ & $\begin{array}{l}-.5356426-01 \\
-.5102561-01 \\
-.4864990-01 \\
-.4642414-01 \\
-.4433656-01\end{array}$ & $\begin{array}{l}.2286112-01 \\
.2142953-01 \\
.2011031-01 \\
.1889296-01 \\
.1776811-01\end{array}$ & $\begin{array}{l}.2064425+01 \\
.2023101+01 \\
.1983311+01 \\
.1944972+01 \\
.1908008+01\end{array}$ & $\begin{array}{l}-.5204981+00 \\
-.5029502+00 \\
-.4863272+00 \\
-.4705649+00 \\
-.4556046+00\end{array}$ & $\begin{array}{l}.1837351+00 \\
.1742980+00 \\
.1655045+00 \\
.1573006+00 \\
.1496379+00\end{array}$ & $\begin{array}{l}-.1044105+00 \\
-.9752208-01 \\
-.9119778-01 \\
-.8538293-01 \\
-.8002898-01\end{array}$ & $\begin{array}{l}-.5012827+01 \\
-.4901630+01 \\
-.4794985+.01 \\
-.4692628+01 \\
-.4594313+01\end{array}$ & $\begin{array}{l}.1782829+01 \\
.1715226+01 \\
.1651461+01 \\
.1591254+01 \\
.1534347+01\end{array}$ & $\begin{array}{l}-.8682165+00 \\
-.8208118+00 \\
-.7767948+00 \\
-.7358704+00 \\
-.6977739+00\end{array}$ & $\begin{array}{l}.6077335+00 \\
.5658876+00 \\
.5275902+00 \\
.4924874+00 \\
.4602655+00\end{array}$ \\
\hline $\begin{array}{l}1.500 \\
1.540 \\
1.580 \\
1.620 \\
1.660\end{array}$ & $\begin{array}{l}-.7108145+00 \\
-.6624364+00 \\
-.6169764+00 \\
-.5741823+00 \\
-.5338302+00\end{array}$ & $\begin{array}{l}.2267013+00 \\
.2154363+00 \\
.2051045+00 \\
.1956018+00 \\
.1868385+00\end{array}$ & $\begin{array}{l}-.4237649-01 \\
-.3880095-01 \\
-.3562990-01 \\
-.3280709-01 \\
-.3028540-01\end{array}$ & $\begin{array}{l}.1672737-01 \\
.1486906-01 \\
.1326673-01 \\
.1187886-01 \\
.1067161-01\end{array}$ & $\begin{array}{l}.1872346+01 \\
.1804668+01 \\
.1741455+01 \\
.1682284+01 \\
.1626782+01\end{array}$ & $\begin{array}{l}-.4413925+00 \\
-.4150191+00 \\
-.3910950+00 \\
-.3693235+00 \\
-.3494512+00\end{array}$ & $\begin{array}{l}.1424723+00 \\
.1294774+00 \\
.1180409+00 \\
.1079355+00 \\
.9897259-01\end{array}$ & $\begin{array}{l}-.7509267-01 \\
-.6632270-01 \\
-.5881056-01 \\
-.5234514-01 \\
-.4675554-01\end{array}$ & $\begin{array}{l}-.4499813+01 \\
-.4321427+01 \\
-.4155951+01 \\
-.4002069+01 \\
-.3858635+01\end{array}$ & $\begin{array}{l}.1480506+01 \\
.1381185+01 \\
.1291783+01 \\
.1211032+01 \\
.1137857+01\end{array}$ & $\begin{array}{l}-.6622667+00 \\
-.5981823+00 \\
-.5421351+00 \\
-.4929097+00 \\
-.4495033+00\end{array}$ & $\begin{array}{l}.4306459+00 \\
.3782501+00 \\
.3336227+00 \\
.2954228+00 \\
.2625712+00\end{array}$ \\
\hline $\begin{array}{l}1.700 \\
1.740 \\
1.780 \\
1.820 \\
1.860\end{array}$ & $\begin{array}{l}-.4957204+00 \\
-.4596741+00 \\
-.4255312+00 \\
-.3931477+00 \\
-.3623936+00\end{array}$ & $\begin{array}{l}.1787367+00 \\
.1712287+00 \\
.1642555+00 \\
.1577652+00 \\
.1517122+00\end{array}$ & $\begin{array}{l}-.2802522-01 \\
-.2599304-01 \\
-.2416043-01 \\
-.2250310-01 \\
-.2100026-01\end{array}$ & $\begin{array}{l}.9617217-02 \\
.8692806-02 \\
.7879414-02 \\
.7161244-02 \\
.6525075-02\end{array}$ & $\begin{array}{l}.1574622+01 \\
.1525513+01 \\
.1479198+01 \\
.1435446+01 \\
.1394053+01\end{array}$ & $\begin{array}{l}-.3312610+00 \\
-.3145659+00 \\
-.2992041+00 \\
-.2850352+00 \\
-.2719369+00\end{array}$ & $\begin{array}{l}.9099455-01 \\
.8386922-01 \\
.7748513-01 \\
.7174783-01 \\
.6657695-01\end{array}$ & $\begin{array}{l}-.4190256-01 \\
-.3767217-01 \\
-.3397044-01 \\
-.3071957-01 \\
-.2785484-01\end{array}$ & $\begin{array}{l}-.3724644+01 \\
-.3599216+01 \\
-.3481572+01 \\
-.3371026+01 \\
-.3266967+01\end{array}$ & $\begin{array}{l}.1071345+01 \\
.1010714+01 \\
.9552905+00 \\
.9044973+00 \\
.8578325+00\end{array}$ & $\begin{array}{l}-.4110839+00 \\
-.3769574+00 \\
-.3465418+00 \\
-.3193471+00 \\
-.2949582+00\end{array}$ & $\begin{array}{l}.2341938+00 \\
.2095785+00 \\
.1881415+00 \\
.1694021+00 \\
.1529622+00\end{array}$ \\
\hline
\end{tabular}


$(m=18)$

\begin{tabular}{|c|c|c|c|c|c|c|c|c|c|c|c|c|}
\hline $\mathrm{T}^{*}$ & B & (1) & (2) & $\mathrm{B}^{*(3)}$ & $\mathrm{T}^{*} \frac{\partial \mathrm{B}^{*(0)}}{\partial \mathrm{T}^{*}}$ & $\mathrm{~T}^{*} \frac{\partial \mathrm{B}^{*(1)}}{\partial \mathrm{T}^{*}}$ & $\mathrm{~T}^{*} \frac{\partial \mathrm{B}^{*(2)}}{\partial \mathrm{T}^{*}}$ & $\mathrm{~T}^{*} \frac{\partial \mathrm{B}^{*(3)}}{\partial \mathrm{T}^{*}}$ & $\mathrm{~T}^{* 2} \frac{\partial^{2} \mathrm{~B}^{*(0)}}{\partial \mathrm{T}^{* 2}}$ & $T^{* 2} \frac{\partial^{2} B^{*(1)}}{\partial T^{* 2}}$ & $\mathrm{~T}^{* 2} \frac{\partial^{2} \mathrm{~B}^{*(2)}}{\partial \mathrm{T}^{* 2}}$ & $\mathrm{~T}^{* 2} \frac{\partial^{2} \mathrm{~B}^{*(3)}}{\partial \mathrm{T}^{* 2}}$ \\
\hline $\begin{array}{l}1.900 \\
1.940 \\
1.980 \\
2.020 \\
2.060\end{array}$ & $\begin{array}{l}-.3331512+00 \\
-.3053142+00 \\
-.2787856+00 \\
-.2534774+00 \\
-.2293090+00\end{array}$ & $\begin{array}{l}.1460564+00 \\
.1407620+00 \\
.1357975+00 \\
.1311346+00 \\
.1267482+00\end{array}$ & $\begin{array}{l}-.1963405-01 \\
-.1838901-01 \\
-.1725177-01 \\
-.1621071-01 \\
-.1525568-01\end{array}$ & $\begin{array}{l}.5959791-02 \\
.5456007-02 \\
.5005763-02 \\
.4602286-02 \\
.4239786-02\end{array}$ & $\begin{array}{l}.1354833+01 \\
.1317621+01 \\
.1282267+01 \\
.1248637+01 \\
.1216607+01\end{array}$ & $\begin{array}{l}-.2598021+00 \\
-.2485367+00 \\
-.2380581+00 \\
-.2282931+00 \\
-.2191771+00\end{array}$ & $\begin{array}{l}.6190383-01 \\
.5766956-01 \\
.5382341-01 \\
.5032154-01 \\
.4712594-01\end{array}$ & $\begin{array}{l}-.2532213-01 \\
-.2307599-01 \\
-.2107808-01 \\
-.1929593-01 \\
-.1770196-01\end{array}$ & $\begin{array}{l}-.3168851+01 \\
-.3076194+01 \\
-.2988561+01 \\
-.2905561+01 \\
-.2826841+01\end{array}$ & $\begin{array}{l}.8148601+00 \\
.7752001+00 \\
.7385197+00 \\
.7045265+00 \\
.6729631+00\end{array}$ & $\begin{array}{l}-.2730227+00 \\
-.2532395+00 \\
-.2353508+00 \\
-.2191349+00 \\
-.2044003+00\end{array}$ & $\begin{array}{l}.1384904+00 \\
.1257099+00 \\
.1143881+00 \\
.1043289+00 \\
.9536635-01\end{array}$ \\
\hline $\begin{array}{l}2.100 \\
2.140 \\
2.180 \\
2.220 \\
2.260\end{array}$ & $\begin{array}{l}-.2062070+00 \\
-.1841040+00 \\
-.1629380+00 \\
-.1426523+00 \\
-.1231944+00\end{array}$ & $\begin{array}{l}.1226158+00 \\
.1187170+00 \\
.1150337+00 \\
.1115493+00 \\
.1082491+00\end{array}$ & $\begin{array}{l}-.1437781-01 \\
-.1356931-01 \\
-.1282332-01 \\
-.1213379-01 \\
-.1149536-01\end{array}$ & $\begin{array}{l}.3913300-02 \\
.3618558-02 \\
.3351872-02 \\
.3110050-02 \\
.2890319-02\end{array}$ & $\begin{array}{l}.1186068+01 \\
.1156917+01 \\
.1129063+01 \\
.1102421+01 \\
.1076915+01\end{array}$ & $\begin{array}{l}-.2106523+00 \\
-.2026676+00 \\
-.1951772+00 \\
-.1881400+00 \\
-.1815192+00\end{array}$ & $\begin{array}{l}.4420352-01 \\
.4152542-01 \\
.3906636-01 \\
.3680415-01 \\
.3471926-01\end{array}$ & \begin{tabular}{|}
$-.1627259-01$ \\
$-.1498767-01$ \\
$-.1382985-01$ \\
$-.1278420-01$ \\
$-.1183779-01$
\end{tabular} & $\begin{array}{l}-.2752086+01 \\
-.2681007+01 \\
-.2613344+01 \\
-.2548860+01 \\
-.2487339+01\end{array}$ & $\begin{array}{l}.6436021+00 \\
.6162418+00 \\
.5907031+00 \\
.5668265+00 \\
.5444694+00\end{array}$ & $\begin{array}{l}-.1909812+00 \\
-.1787337+00 \\
-.1675321+00 \\
-.1572667+00 \\
-.1478412+00\end{array}$ & $\begin{array}{l}.8735941-01 \\
.8018776-01 \\
.7374840-01 \\
.6795289-01 \\
.6272503-01\end{array}$ \\
\hline $\begin{array}{l}2.300 \\
2.340 \\
2.380 \\
2.420 \\
2.460\end{array}$ & $\begin{array}{l}-.1045159+00 \\
-.8657231-01 \\
-.6932218-01 \\
-.5272723-01 \\
-.3675190-01\end{array}$ & $\begin{array}{l}.1051196+00 \\
.1021485+00 \\
.9932468-01 \\
.9663804-01 \\
.9407927-01\end{array}$ & $\begin{array}{l}-.1090330-01 \\
-.1035336-01 \\
-.9841789-02 \\
-.9365219-02 \\
-.8920636-02\end{array}$ & & & & & & & $\begin{array}{l}.5235043+00 \\
.5038166+00 \\
.4853033+00 \\
.4678718+00 \\
.4514381+00\end{array}$ & & $\begin{array}{l}.5799894-01 \\
.5371753-01 \\
.4983115-01 \\
.4629654-01 \\
.4307585-01\end{array}$ \\
\hline $\begin{array}{l}2.500 \\
2.600 \\
2.700 \\
2.800 \\
2.900\end{array}$ & $\begin{array}{r}-.2136313-01 \\
.1473918-01 \\
.4778841-01 \\
.7814456-01 \\
.1061135+00\end{array}$ & & \begin{tabular}{|}
$-.8505338-02$ \\
$-.7579850-02$ \\
$-.6790931-02$ \\
$-.6113725-02$ \\
$-.5528675-02$
\end{tabular} & & & & & & & & & $\begin{array}{l}.4013595-01 \\
.3383193-01 \\
.2874122-01 \\
.2459174-01 \\
.2118028-01\end{array}$ \\
\hline $\begin{array}{l}3.000 \\
3.100 \\
3.200 \\
3.300 \\
3.400\end{array}$ & $\begin{array}{l}.1319568+00 \\
.1558995+00 \\
.1781360+00 \\
.1988355+00 \\
.2181455+00\end{array}$ & $\begin{array}{l}.6222111-01 \\
.5939574-01 \\
.5679694-01\end{array}$ & \begin{tabular}{|}
$-.5020240-02$ \\
$-.4575963-02$ \\
$-.4185770-02$ \\
$-.3841454-02$ \\
$-.3536279-02$
\end{tabular} & & & & & & & & & $\begin{array}{l}.1835326-01 \\
.1599328-01 \\
.1400973-01 \\
.1233197-01 \\
.1090443-01\end{array}$ \\
\hline $\begin{array}{l}3.500 \\
3.600 \\
3.700 \\
3.800 \\
3.900\end{array}$ & $\begin{array}{l}.2361956+00 \\
.2530997+00 \\
.2689588+00 \\
.2838621+00 \\
.2978893+00\end{array}$ & $\begin{array}{l}.5012489-01 \\
.4821269-01 \\
.4643091-01\end{array}$ & $\begin{array}{l}-.3264680-02 \\
-.3022028-02 \\
-.2804451-02 \\
-.2608695-02 \\
-.2432005-02\end{array}$ & & $\begin{array}{l}.6110751+00 \\
.5891686+00 \\
.5685839+00 \\
.5492046+00 \\
.5309278+00\end{array}$ & $\begin{array}{l}-.8064884-01 \\
-.7683832-01 \\
-.7333368-01 \\
-.7010149-01 \\
-.6711286-01\end{array}$ & & $\begin{array}{l}-.1958295-02 \\
-.1752822-02 \\
-.1574507-02 \\
-.1419079-02 \\
-.1283036-02\end{array}$ & & $\begin{array}{l}.2198056+00 \\
.2082955+00 \\
.1977738+00 \\
.1881269+00 \\
.1792572+00\end{array}$ & & $\begin{array}{l}.9683088-02 \\
.8632724-02 \\
.7725010-02 \\
.6936982-02 \\
.6249913-02\end{array}$ \\
\hline $\begin{array}{l}4.000 \\
4.100 \\
4.200 \\
4.300 \\
4.400\end{array}$ & $\begin{array}{l}.3111115+00 \\
.3235925+00 \\
.3353894+00 \\
.3465538+00 \\
.3571322+00\end{array}$ & $\begin{array}{l}.4175096-01 \\
.4038038-01 \\
.3909101-01\end{array}$ & $\begin{array}{l}-.2272042-02 \\
-.2126807-02 \\
-.1994589-02 \\
-.1873912-02 \\
-.1763501-02\end{array}$ & & & $\begin{array}{l}-.6434271-01 \\
-.6176916-01 \\
-.5937308-01 \\
-.5713764-01 \\
-.5504805-01\end{array}$ & & $\begin{array}{l}-.1163495-02 \\
-.1058064-02 \\
-.9647531-03 \\
-.8818937-03 \\
-.8080834-03\end{array}$ & $\begin{array}{l}-.1185200+01 \\
-.1149109+01 \\
-.1114999+01 \\
-.1082711+01 \\
-.1052104+01\end{array}$ & $\begin{array}{l}.1710807+00 \\
.1635243+00 \\
.1565246+00 \\
.1500261+00 \\
.1439802+00\end{array}$ & $\begin{array}{l}-.2352871-01 \\
-.2184691-01 \\
-.2032921-01 \\
-.1895569-01 \\
-.1770928-01\end{array}$ & $\begin{array}{l}.5119860-02 \\
.4653668-02 \\
.4241081-02 \\
.3874742-02\end{array}$ \\
\hline $\begin{array}{l}4.500 \\
4.600 \\
4.700 \\
4.800 \\
4.900\end{array}$ & $\begin{array}{l}.3671669+00 \\
.3766961+00 \\
.3857546+00 \\
.3943742+00 \\
.4025838+00\end{array}$ & $\begin{array}{l}.3787606-01 \\
.3672949-01 \\
.3564586-01 \\
.3462028-01 \\
.3364835-01\end{array}$ & $\begin{array}{l}-.1662248-02 \\
-.1569192-02 \\
-.1483488-02 \\
-.1404400-02 \\
-.1331276-02\end{array}$ & & & & $\begin{array}{l}.4365205-02 \\
.4105450-02 \\
.3867204-02 \\
.3648221-02 \\
.3446538-02\end{array}$ & & $\begin{array}{l}-.1023049+01 \\
-.9954320+00 \\
-.9691480+00 \\
-.9441030+00 \\
-.9202115+00\end{array}$ & $\begin{array}{l}.1383443+00 \\
.1330804+00 \\
.1281553+00 \\
.1235391+00 \\
.1192055+00\end{array}$ & $\begin{array}{l}-.1657530-01 \\
-.1554109-01 \\
-.1459567-01 \\
-.1372950-01 \\
-.1293425-01\end{array}$ & $\begin{array}{l}.3548459-02 \\
.3256994-02 \\
.2995901-02 \\
.2761390-02 \\
.2550214-02\end{array}$ \\
\hline $\begin{array}{l}5.000 \\
5.100 \\
5.200 \\
5.300 \\
5.400\end{array}$ & $\begin{array}{l}.4104099+00 \\
.4178769+00 \\
.4250072+00 \\
.4318212+00 \\
.4383379+00\end{array}$ & $\begin{array}{l}.3272610-01 \\
.3184994-01 \\
.3101659-01 \\
.3022308-01 \\
.2946670-01\end{array}$ & $\begin{array}{l}-.1263545-02 \\
-.1200700-02 \\
-.1142291-02 \\
-.1087919-02 \\
-.1037228-02\end{array}$ & $\begin{array}{l}.1395018-03 \\
.1299583-03 \\
.1212567-03 \\
.1133071-03 \\
.1060305-03\end{array}$ & $\begin{array}{l}.3821348+00 \\
.3720479+00 \\
.3623795+00 \\
.3531039+00 \\
.3441978+00\end{array}$ & $\begin{array}{l}-.4493175-01 \\
-.4356626-01 \\
-.4227386-01 \\
-.4104909-01 \\
-.3988702-01\end{array}$ & $\begin{array}{l}.3260429-02 \\
.3088374-02 \\
.2929032-02 \\
.2781213-02 \\
.2643861-02\end{array}$ & $\begin{array}{l}-.4998860-03 \\
-.4644338-03 \\
-.4322039-03 \\
-.4028430-03 \\
-.3760425-03\end{array}$ & $\begin{array}{l}-.8973954+00 \\
-.8755838+00 \\
-.8547116+00 \\
-.8347194+00 \\
-.8155527+00\end{array}$ & $\begin{array}{l}.1151308+00 \\
.1112938+00 \\
.1076756+00 \\
.1042589+00 \\
.1010285+00\end{array}$ & $\begin{array}{l}-.1220265-01 \\
-.1152829-01 \\
-.1090555-01 \\
-.1032945-01 \\
-.9795613-02\end{array}$ & $\begin{array}{l}.2359589-02 \\
.2187113-02 \\
.2030710-02 \\
.1888578-02 \\
.1759150-02\end{array}$ \\
\hline $\begin{array}{l}5.500 \\
5.600 \\
5.800 \\
6.000 \\
6.200\end{array}$ & $\begin{array}{l}.4445749+00 \\
.4505482+00 \\
.4617627+00 \\
.4720888+00 \\
.4816190+00\end{array}$ & $\begin{array}{l}.2874499-01 \\
.2805569-01 \\
.2676616-01 \\
.2558349-01 \\
.2449525-01\end{array}$ & $\left|\begin{array}{|}-.9898983-03 \\
-.9456457-03 \\
-.8653726-03 \\
-.7946516-03 \\
-.7320502-03\end{array}\right|$ & $\begin{array}{l}.9935796-04 \\
.9322849-04 \\
.8239022-04 \\
.7315578-04 \\
.6524112-04\end{array}$ & $\begin{array}{l}.3356394+00 \\
.3274088+00 \\
.3118582+00 \\
.2974137+00 \\
.2839614+00\end{array}$ & $\begin{array}{l}-.3878317-01 \\
-.3773346-01 \\
-.3578196-01 \\
-.3400647-01 \\
-.3238514-01\end{array}$ & $\begin{array}{l}.2516037-02 \\
.2396903-02 \\
.2181780-02 \\
.1993371-02 \\
.1827527-02\end{array}$ & $\begin{array}{l}-.3515326-03 \\
-.3290767-03 \\
-.2895181-03 \\
-.2559766-03 \\
-.2273612-03\end{array}$ & $\begin{array}{l}-.7971612+00 \\
-.7794988+00 \\
-.7461943+00 \\
-.7153358+00 \\
-.6866629+00\end{array}$ & $\begin{array}{l}.9797034-01 \\
.9507175-01 \\
.8970845-01 \\
.8485859-01 \\
.8045543-01\end{array}$ & $\begin{array}{l}-.9300119-02 \\
-.8839499-02 \\
-.8010822-02 \\
-.7288515-02 \\
-.6655610-02\end{array}$ & $\begin{array}{l}.1641059-02 \\
.1533109-02 \\
.1343555-02 \\
.1183505-02 \\
.1047504-02\end{array}$ \\
\hline
\end{tabular}


$(m=18)$

\begin{tabular}{|c|c|c|c|c|c|c|c|c|c|c|c|c|}
\hline $\mathrm{T}^{*}$ & B & 1) & 2) & (3) & $\mathrm{T}^{*} \frac{\partial \mathrm{B}^{*(0)}}{\partial \mathrm{T}^{*}}$ & $\mathrm{~T}^{*} \frac{\partial \mathrm{B}^{*(1)}}{\partial \mathrm{T}^{*}}$ & $\mathrm{~T}^{*} \frac{\partial \mathrm{B}^{*(2)}}{\partial \mathrm{T}^{*}}$ & $\mathrm{~T}^{*} \frac{\partial \mathrm{B}^{*(3)}}{\partial \mathrm{T}^{*}}$ & $\mathrm{~T}^{* 2} \frac{\partial^{2} \mathrm{~B}^{*(0)}}{\partial \mathrm{T}^{* 2}}$ & $T^{* 2} \frac{\partial^{2} B^{*(1)}}{\partial T^{* 2}}$ & $T^{* 2} \frac{\partial^{2} B^{*(2)}}{\partial T^{* 2}}$ & $\mathrm{~T}^{* 2} \frac{\partial^{2} \mathrm{~B}^{*(3)}}{\partial \mathrm{T}^{* 2}}$ \\
\hline $\begin{array}{l}6.400 \\
6.600 \\
6.800 \\
7.000 \\
7.200\end{array}$ & $\begin{array}{l}.4904338+00 \\
.4986035+00 \\
.5061894+00 \\
.5132457+00 \\
.5198201+00\end{array}$ & $\begin{array}{l}.2349086-01 \\
.2256123-01 \\
.2169850-01 \\
.2089589-01 \\
.2014748-01\end{array}$ & $\begin{array}{l}-.6763913-03 \\
-.6267012-03 \\
-.5821693-03 \\
-.5421165-03 \\
-.5059706-03\end{array}$ & $\begin{array}{l}.5842016-04 \\
.5251149-04 \\
.4736847-04 \\
.4287170-04 \\
.3892335-04\end{array}$ & $\begin{array}{l}.2714023+00 \\
.2596503+00 \\
.2486300+00 \\
.2382752+00 \\
.2285273+00\end{array}$ & $\begin{array}{l}-.3089950-01 \\
-.2953385-01 \\
-.2827475-01 \\
-.2711067-01 \\
-.2603163-01\end{array}$ & $\begin{array}{l}.1680863-02 \\
.1550595-02 \\
.1434419-02 \\
.1330416-02 \\
.1236978-02\end{array}$ & $\begin{array}{l}-.2028083-03 \\
-.1816283-03 \\
-.1632666-03 \\
-.1472734-03 \\
-.1332822-03\end{array}$ & $\begin{array}{l}-.6599508+00 \\
-.6350048+00 \\
-.6116549+00 \\
-.5897523+00 \\
-.5691661+00\end{array}$ & $\begin{array}{l}.7644298-01 \\
.7277395-01 \\
.6940814-01 \\
.6631123-01 \\
.6345370-01\end{array}$ & $\begin{array}{l}-.6098329-02 \\
-.5605402-02 \\
-.5167542-02 \\
-.4777052-02 \\
-.4427508-02\end{array}$ & $\begin{array}{l}.9312524-03 \\
.8313335-03 \\
.7450090-03 \\
.6700688-03 \\
.6047166-03\end{array}$ \\
\hline $\begin{array}{l}7.400 \\
7.600 \\
7.800 \\
8.000 \\
8.200\end{array}$ & $\begin{array}{l}.5259549+00 \\
.5316878+00 \\
.5370524+00 \\
.5420786+00 \\
.5467934+00\end{array}$ & $\begin{array}{l}.1944808-01 \\
.1879314-01 \\
.1817866-01 \\
.1760107-01 \\
.1705724-01\end{array}$ & $\begin{array}{l}-.4732469-03 \\
-.4435330-03 \\
-.4164759-03 \\
-.3917724-03 \\
-.3691611-03\end{array}$ & $\begin{array}{l}.3544274-04 \\
.3236296-04 \\
.2962822-04 \\
.2719175-04 \\
.2501416-04\end{array}$ & $\begin{array}{l}.2193345+00 \\
.2106508+00 \\
.2024351+00 \\
.1946505+00 \\
.1872640+00\end{array}$ & $\begin{array}{l}-.2502898-01 \\
-.2409516-01 \\
-.2322359-01 \\
-.2240844-01 \\
-.2164460-01\end{array}$ & $\begin{array}{l}.1152750-02 \\
.1076584-02 \\
.1007504-02 \\
.9446748-03 \\
.8873780-03\end{array}$ & $\begin{array}{l}-.1209918-03 \\
-.1101534-03 \\
-.1005604-03 \\
-.9204029-04 \\
-.8444829-04\end{array}$ & $\begin{array}{l}-.5497810+00 \\
-.5314945+00 \\
-.5142155+00 \\
-.4978628+00 \\
-.4823638+00\end{array}$ & $\begin{array}{l}.6081011-01 \\
.5835839-01 \\
.5607931-01 \\
.5395605-01 \\
.5197386-01\end{array}$ & $\begin{array}{l}-.4113520-02 \\
-.3830543-02 \\
-.3574722-02 \\
-.3342772-02 \\
-.3131884-02\end{array}$ & $\begin{array}{l}.5474829-03 \\
.4971582-03 \\
.4527412-03 \\
.4133981-03 \\
.3784318-03\end{array}$ \\
\hline $\begin{array}{l}8.400 \\
8.600 \\
8.800 \\
9.000 \\
9.200\end{array}$ & $\begin{array}{l}.5512211+00 \\
.5553835+00 \\
.5593003+00 \\
.5629896+00 \\
.5664676+00\end{array}$ & $\begin{array}{l}.1654436-01 \\
.1605990-01 \\
.1560164-01 \\
.1516753-01 \\
.1475577-01\end{array}$ & $\begin{array}{l}-.3484154-03 \\
-.3293383-03 \\
-.3117577-03 \\
-.2955232-03 \\
-.2805025-03\end{array}$ & & $\begin{array}{l}.1802460+00 \\
.1735695+00 \\
.1672103+00 \\
.1611463+00 \\
.1553575+00\end{array}$ & $\begin{array}{l}-.2092754-01 \\
-.2025324-01 \\
-.1961809-01 \\
-.1901891-01 \\
-.1845282-01\end{array}$ & $\begin{array}{l}.8349952-03 \\
.7869906-03 \\
.7428985-03 \\
.7023126-03 \\
.6648775-03\end{array}$ & $\begin{array}{l}-.7766228-04 \\
-.7157879-04 \\
-.6610984-04 \\
-.6118025-04 \\
-.5672555-04\end{array}$ & $\begin{array}{l}-.4676530+00 \\
-.4536716+00 \\
-.4403667+00 \\
-.4276901+00 \\
-.4155983+00\end{array}$ & $\begin{array}{l}.5011975-01 \\
.4838221-01 \\
.4675105-01 \\
.4521719-01 \\
.4377255-01\end{array}$ & $\begin{array}{l}-.2939642-02 \\
-.2763960-02 \\
-.2603033-02 \\
-.2455291-02 \\
-.2319364-02\end{array}$ & $\begin{array}{l}.3472557-03 \\
.3193748-03 \\
.2943687-03 \\
.2718795-03 \\
.2516011-03\end{array}$ \\
\hline $\begin{array}{r}9.400 \\
9.600 \\
9.800 \\
10.000 \\
10.200\end{array}$ & & $\begin{array}{l}.1436472-01 \\
.1399287-01 \\
.1363888-01 \\
.1330152-01 \\
.1297966-01\end{array}$ & $\begin{array}{l}-.2665790-03 \\
-.2536497-03 \\
-.2416234-03 \\
-.2304187-03 \\
-.2199634-03\end{array}$ & & $\begin{array}{l}.1498257+00 \\
.1445341+00 \\
.1394675+00 \\
.1346119+00 \\
.1299544+00\end{array}$ & $\begin{array}{l}-.1791724-01 \\
-.1740984-01 \\
-.1692854-01 \\
-.1647142-01 \\
-.1603678-01\end{array}$ & $\begin{array}{l}.6302810-03 \\
.5982481-03 \\
.5685360-03 \\
.5409297-03 \\
.5152380-03\end{array}$ & $\begin{array}{l}-.5269025-04 \\
-.4902644-04 \\
-.4569257-04 \\
-.4265254-04 \\
-.3987485-04\end{array}$ & $\begin{array}{l}-.4040516+00 \\
-.3930138+00 \\
-.3824520+00 \\
-.3723360+00 \\
-.3626380+00\end{array}$ & $\begin{array}{l}.4240987-01 \\
.4112266-01 \\
.3990506-01 \\
.3875180-01 \\
.3765811-01\end{array}$ & $\begin{array}{l}-.2194051-02 \\
-.2078301-02 \\
-.1971184-02 \\
-.1871881-02 \\
-.1779666-02\end{array}$ & $\begin{array}{l}.2332706-03 \\
.2166616-03 \\
.2015782-03 \\
.1878506-03 \\
.1753309-03\end{array}$ \\
\hline $\begin{array}{l}10.400 \\
10.600 \\
10.800 \\
11.000 \\
11.400\end{array}$ & $\begin{array}{l}.5836429+00 \\
.5859920+00 \\
.5882186+00 \\
.5903299+00 \\
.5942339+00\end{array}$ & $\begin{array}{l}.1267230-01 \\
.1237848-01 \\
.1209735-01 \\
.1182813-01 \\
.1132255-01\end{array}$ & $\begin{array}{l}-.2101929-03 \\
-.2010494-03 \\
-.1924808-03 \\
-.1844406-03 \\
-.1697808-03\end{array}$ & & $\begin{array}{l}.1254832+00 \\
.1211875+00 \\
.1170570+00 \\
.1130826+00 \\
.1055679+00\end{array}$ & $\begin{array}{l}-.1562303-01 \\
-.1522876-01 \\
-.1485266-01 \\
-.1449355-01 \\
-.1382198-01\end{array}$ & $\begin{array}{l}.4912908-03 \\
.4689362-03 \\
.4480384-03 \\
.4284754-03 \\
.3929268-03\end{array}$ & $\begin{array}{l}-.3733195-04 \\
-.3499968-04 \\
-.3285678-04 \\
-.3088452-04 \\
-.2738748-04\end{array}$ & $\begin{array}{l}-.3533326+00 \\
-.3443964+00 \\
-.3358079+00 \\
-.3275470+00 \\
-.3119357+00\end{array}$ & $\begin{array}{l}.3661967-01 \\
.3563256-01 \\
.3469321-01 \\
.3379837-01 \\
.3213057-01\end{array}$ & & $\begin{array}{l}.1638902-03 \\
.1534156-03 \\
.1438079-03 \\
.1349798-03 \\
.1193638-03\end{array}$ \\
\hline $\begin{array}{l}11.800 \\
12.200 \\
12.600 \\
13.000 \\
13.400\end{array}$ & $\begin{array}{l}.5977533+00 \\
.6009305+00 \\
.6038021+00 \\
.6063999+00 \\
.6087517+00\end{array}$ & $\begin{array}{l}.1085658-01 \\
.1042584-01 \\
.1002655-01 \\
.9655446-02 \\
.9309696-02\end{array}$ & $\begin{array}{l}-.1567794-03 \\
-.1451977-03 \\
-.1348381-03 \\
-.1255361-03 \\
-.1171537-03\end{array}$ & & $\begin{array}{l}.9858153-01 \\
.9207001-01 \\
.8598683-01 \\
.8029140-01 \\
.7494808-01\end{array}$ & $\begin{array}{l}-.1320638-01 \\
-.1264019-01 \\
-.1211787-01 \\
-.1163462-01 \\
-.1118634-01\end{array}$ & $\begin{array}{l}.3615372-03 \\
.3336910-03 \\
.3088814-03 \\
.2866882-03 \\
.2667606-03\end{array}$ & $\begin{array}{l}-.2439719-04 \\
-.2182545-04 \\
-.1960178-04 \\
-.1766942-04 \\
-.1598235-04\end{array}$ & $\begin{array}{l}-.2974309+00 \\
-.2839187+00 \\
-.2713005+00 \\
-.2594903+00 \\
-.2484128+00\end{array}$ & $\begin{array}{l}.3060826-01 \\
.2921385-01 \\
.2793239-01 \\
.2675114-01 \\
.2565916-01\end{array}$ & $\begin{array}{l}-.1232530-02 \\
-.1134354-02 \\
-.1047168-02 \\
-.9694169-03 \\
-.8998099-03\end{array}$ & $\begin{array}{l}.1060515-03 \\
.9463567-04 \\
.8479216-04 \\
.7626071-04 \\
.6883087-04\end{array}$ \\
\hline $\begin{array}{l}13.800 \\
14.200 \\
14.600 \\
15.000 \\
16.000\end{array}$ & $\begin{array}{l}.6108820+00 \\
.6128121+00 \\
.6145609+00 \\
.6161452+00 \\
.6194801+00\end{array}$ & $\begin{array}{l}.8986836-02 \\
.8684706-02 \\
.8401404-02 \\
.8135256-02 \\
.7535581-02\end{array}$ & $\begin{array}{l}-.1095745-03 \\
-.1027000-03 \\
-.9644608-04 \\
-.9074086-04 \\
-.7849267-04\end{array}$ & $\begin{array}{l}.4505169-05 \\
.4109680-05 \\
.3759222-05 \\
.3447568-05 \\
.2805905-05\end{array}$ & $\begin{array}{l}.6992548-01 \\
.6519582-01 \\
.6073445-01 \\
.5651942-01 \\
.4693567-01\end{array}$ & $\begin{array}{l}-.1076947-01 \\
-.1038088-01 \\
-.1001788-01 \\
-.9678068-02 \\
-.8916829-02\end{array}$ & $\begin{array}{l}.2488043-03 \\
.2325708-03 \\
.2178492-03 \\
.2044596-03 \\
.1758537-03\end{array}$ & $\begin{array}{l}-.1450297-04 \\
-.1320036-04 \\
-.1204897-04 \\
-.1102752-04 \\
-.8932545-05\end{array}$ & $\begin{array}{l}-.2380019+00 \\
-.2281991+00 \\
-.2189526+00 \\
-.2102164+00 \\
-.1903492+00\end{array}$ & $\begin{array}{l}.2464702-01 \\
.2370656-01 \\
.2283064-01 \\
.2201305-01 \\
.2019000-01\end{array}$ & $\begin{array}{l}-.8372657-03 \\
-.7808750-03 \\
-.7298688-03 \\
-.6835930-03 \\
-.5851244-03\end{array}$ & $\begin{array}{l}.6233128-04 \\
.5662145-04 \\
.5158550-04 \\
.4712728-04 \\
.3801432-04\end{array}$ \\
\hline $\begin{array}{l}17.000 \\
18.000 \\
19.000 \\
20.000 \\
21.000\end{array}$ & $\begin{array}{l}.6220676+00 \\
.6240542+00 \\
.6255529+00 \\
.6266521+00 \\
.6274220+00\end{array}$ & $\begin{array}{l}.7015150-02 \\
.6559454-02 \\
.6157293-02 \\
.5799887-02 \\
.5480266-02\end{array}$ & $\begin{array}{l}-.6854813-04 \\
-.6036704-04 \\
-.5355807-04 \\
-.4783226-04 \\
-.4297262-04\end{array}$ & $\begin{array}{l}.2314389-05 \\
.1931547-05 \\
.1628918-05 \\
.1386538-05 \\
.1190117-05\end{array}$ & $\begin{array}{l}.3851824-01 \\
.3106897-01 \\
.2443215-01 \\
.1848374-01 \\
.1312368-01\end{array}$ & $\begin{array}{l}-.8261249-02 \\
-.7691191-02 \\
-.7191271-02 \\
-.6749543-02 \\
-.6356598-02\end{array}$ & $\begin{array}{l}.1527804-03 \\
.1339122-03 \\
.1182947-03 \\
.1052280-03 \\
.9418976-04\end{array}$ & $\begin{array}{l}-.7336180-05 \\
-.6098685-05 \\
-.5124734-05 \\
-.4347802-05 \\
-.3720515-05\end{array}$ & $\begin{array}{l}-.1728911+00 \\
-.1574295+00 \\
-.1436411+00 \\
-.1312689+00 \\
-.1201060+00\end{array}$ & $\begin{array}{l}.1862974-01 \\
.1728064-01 \\
.1610359-01 \\
.1506843-01 \\
.1415156-01\end{array}$ & $\begin{array}{l}-.5061323-03 \\
-.4418574-03 \\
-.3888984-03 \\
-.3447752-03 \\
-.3076466-03\end{array}$ & $\begin{array}{l}.3110207-04 \\
.2576609-04 \\
.2158254-04 \\
.1825701-04 \\
.1558074-04\end{array}$ \\
\hline $\begin{array}{l}22.000 \\
23.000 \\
24.000 \\
25.000 \\
26.000\end{array}$ & $\begin{array}{l}.6279187+00 \\
.6281874+00 \\
.6282651+00 \\
.6281821+00 \\
.6279634+00\end{array}$ & $\begin{array}{l}.5192821-02 \\
.4932993-02 \\
.4697040-02 \\
.4481860-02 \\
.4284863-02\end{array}$ & $\begin{array}{l}-.3881368-04 \\
-.3522753-04 \\
-.3211406-04 \\
-.2939406-04 \\
-.2700416-04\end{array}$ & $\begin{array}{l}.1029248-05 \\
.8962281-06 \\
.7852743-06 \\
.6919874-06 \\
.6129805-06\end{array}$ & $\begin{array}{r}.8270375-02 \\
.3856636-02 \\
-.1733935-03 \\
-.3866513-02 \\
-.7262231-02\end{array}$ & $\begin{array}{l}-.6004930-02 \\
-.5688480-02 \\
-.5402307-02 \\
-.5142344-02 \\
-.4905212-02\end{array}$ & $\begin{array}{l}.8478400-04 \\
.7670637-04 \\
.6971988-04 \\
.6363784-04 \\
.5831165-04\end{array}$ & $\begin{array}{l}-.3208525-05 \\
-.2786518-05 \\
-.2435562-05 \\
-.2141307-05 \\
-.1892745-05\end{array}$ & $\begin{array}{l}-.1099841+00 \\
-.1007648+00 \\
-.9233300-01 \\
-.8459267-01 \\
-.7746265-01\end{array}$ & $\begin{array}{l}.1333427-01 \\
.1260153-01 \\
.1194117-01 \\
.1134320-01 \\
.1079938-01\end{array}$ & $\begin{array}{l}-.2761234-03 \\
-.2491427-03 \\
-.2258800-03 \\
-.2056886-03 \\
-.1880556-03\end{array}$ & $\begin{array}{l}.1340295-04 \\
.1161294-04 \\
.1012820-04 \\
.8886394-05 \\
.7839828-05\end{array}$ \\
\hline $\begin{array}{l}27.000 \\
28.000 \\
29.000 \\
30.000 \\
32.000\end{array}$ & $\begin{array}{l}.6276298+00 \\
.6271988+00 \\
.6266849+00 \\
.6261007+00 \\
.6247614+00\end{array}$ & $\begin{array}{l}.4103869-02 \\
.3937030-02 \\
.3782771-02 \\
.3639740-02 \\
.3382848-02\end{array}$ & $\begin{array}{l}-.2489324-04 \\
-.2301965-04 \\
-.2134924-04 \\
-.1985374-04 \\
-.1729721-04\end{array}$ & $\begin{array}{l}.5456173-06 \\
.4878252-06 \\
.4379594-06 \\
.3947030-06 \\
.3239678-06\end{array}$ & $\begin{array}{l}-.1039413-01 \\
-.1329095-01 \\
-.1597742-01 \\
-.1847491-01 \\
-.2297503-01\end{array}$ & $\begin{array}{l}-.4688084-02 \\
-.4488576-02 \\
-.4304664-02 \\
-.4134619-02 \\
-.3830399-02\end{array}$ & $\begin{array}{l}.5362186-04 \\
.4947161-04 \\
.4578170-04 \\
.4248687-04 \\
.3687484-04\end{array}$ & $\begin{array}{l}-.1681334-05 \\
-.1500380-05 \\
-.1344584-05 \\
-.1209717-05 \\
-.9897980-06\end{array}$ & $\begin{array}{l}-.7087403-01 \\
-.6476794-01 \\
-.5909372-01 \\
-.5380758-01 \\
-.4425208-01\end{array}$ & $\begin{array}{l}.1030283-01 \\
.9847765-02 \\
.9429314-02 \\
.9043319-02 \\
.8354963-02\end{array}$ & $\begin{array}{l}-.1725700-03 \\
-.1588999-03 \\
-.1467743-03 \\
-.1359711-03 \\
-.1176261-03\end{array}$ & $\begin{array}{l}.6951613-05 \\
.6192908-05 \\
.5540946-05 \\
.4977595-05 \\
.4061264-05\end{array}$ \\
\hline
\end{tabular}


$(m=18)$

\begin{tabular}{|c|c|c|c|c|c|c|c|c|c|c|c|c|}
\hline$T^{*}$ & $\mathrm{~B}^{*(0)}$ & (1) & $\mathrm{B}^{*(2)}$ & 3) & $\mathrm{T}^{*} \frac{\partial \mathrm{B}^{*(0)}}{\partial \mathrm{T}^{*}}$ & $\mathrm{~T}^{*} \frac{\partial \mathrm{B}^{*(1)}}{\partial \mathrm{T}^{*}}$ & $\mathrm{~T}^{*} \frac{\partial \mathrm{B}^{*(2)}}{\partial \mathrm{T}^{*}}$ & $\mathrm{~T}^{*} \frac{\partial \mathrm{B}^{*(3)}}{\partial \mathrm{T}^{*}}$ & $T^{* 2} \frac{\partial^{2} B^{*(0)}}{\partial T^{* 2}}$ & $\mathrm{~T}^{* 2} \frac{\partial^{2} \mathrm{~B}^{*(1)}}{\partial \mathrm{T}^{* 2}}$ & $\mathrm{~T}^{* 2} \frac{\partial^{2} \mathrm{~B}^{*(2)}}{\partial \mathrm{T}^{* 2}}$ & $* 2 \frac{\partial^{2} B^{*(3)}}{\partial T^{* 2}}$ \\
\hline $\begin{array}{l}34.000 \\
36.000 \\
38.000 \\
40.000 \\
42.000\end{array}$ & $\begin{array}{l}.6232478+00 \\
.6216090+00 \\
.6198819+00 \\
.6180939+00 \\
.6162660+00\end{array}$ & $\begin{array}{l}.3158739-02 \\
.2961586-02 \\
.2786859-02 \\
.2630980-02 \\
.2491091-02\end{array}$ & $\begin{array}{l}.1520353-04 \\
.1346761-04 \\
.1201251-04 \\
.1078089-04 \\
.9729291-05\end{array}$ & $\begin{array}{l}.2692626-06 \\
.2262812-06 \\
.1920360-06 \\
.1644094-06 \\
.1418720-06\end{array}$ & $\begin{array}{l}-.2691402-01 \\
-.3038704-01 \\
-.3346899-01 \\
-.3621968-01 \\
-.3868739-01\end{array}$ & $\begin{array}{l}-.3566274-02 \\
-.3334926-02 \\
-.3130700-02 \\
-.2949158-02 \\
-.2786775-02\end{array}$ & $\begin{array}{l}.3229965-04 \\
.2852188-04 \\
.2536718-04 \\
.2270622-04 \\
.2044147-04\end{array}$ & $\begin{array}{l}-.8203190-06 \\
-.6875893-06 \\
-.5821483-06 \\
-.4973152-06 \\
-.4282814-06\end{array}$ & $\begin{array}{l}-.3585046-01 \\
-.2840814-01 \\
-.2177196-01 \\
-.1581966-01 \\
-.1045250-01\end{array}$ & $\begin{array}{l}.7759704-02 \\
.7240183-02 \\
.6783060-02 \\
.6377923-02 \\
.6016533-02\end{array}$ & $\begin{array}{l}-.1027275-03 \\
-.9046827-04 \\
-.8026356-04 \\
-.7168119-04 \\
-.6439645-04\end{array}$ & $\begin{array}{l}.3357318-05 \\
.2807585-05 \\
.2372013-05 \\
.2022410-05 \\
.1738545-05\end{array}$ \\
\hline $\begin{array}{l}44.000 \\
46.000 \\
48.000 \\
50.000 \\
52.000\end{array}$ & $\begin{array}{l}.6106842+00 \\
.6088230+00 \\
.6069721+00\end{array}$ & & & & & & & $\begin{array}{l}-.3715254-06 \\
-.3244281-06 \\
-.2850140-06 \\
-.2517738-06 \\
-.2235411-06\end{array}$ & $\begin{array}{r}-.5589842-02 \\
-.1165186-02 \\
.2876799-02 \\
.6582527-02 \\
.9991221-02\end{array}$ & & $\begin{array}{l}-.5816168-04 \\
-.5278535-04 \\
-.4811755-04 \\
-.4403958-04 \\
-.4045651-04\end{array}$ & $\begin{array}{l}.1505642-05 \\
.1312742-05 \\
.1151596-05 \\
.1015917-05 \\
.9008557-06\end{array}$ \\
\hline $\begin{array}{l}54.000 \\
56.000 \\
58.000 \\
60.000 \\
64.000\end{array}$ & & & & & $-.4934592-01$ & & & & & & $\begin{array}{l}-.3729172-04 \\
-.3448280-04 \\
-.3197853-04 \\
-.2973654-04 \\
-.2590410-04\end{array}$ & $\begin{array}{l}.8026313-06 \\
.7182658-06 \\
.6453924-06 \\
.5821139-06 \\
.4784947-06\end{array}$ \\
\hline $\begin{array}{l}68.000 \\
72.000 \\
76.000 \\
80.000 \\
84.000\end{array}$ & & & & & & $\begin{array}{l}-.1606534-02 \\
-.1506253-02 \\
-.1417375-02 \\
-.1338081-02 \\
-.1266920-02\end{array}$ & & & & & & $\begin{array}{l}.3982199-06 \\
.3350514-06 \\
.2846518-06 \\
.2439413-06 \\
.2106916-06\end{array}$ \\
\hline $\begin{array}{r}88.000 \\
92.000 \\
96.000 \\
100.000 \\
105.000\end{array}$ & $\begin{array}{l}.5775063+00 \\
.5747157+00 \\
.5720117+00 \\
.5693906+00 \\
.5662249+00\end{array}$ & & $\begin{array}{l}-.2107104-05 \\
-.1924521-05 \\
-.1764756-05 \\
-.1624156-05 \\
-.1470917-05\end{array}$ & & & & $\begin{array}{l}9341-05 \\
1436-05 \\
1321-05 \\
1268-05 \\
5665-05\end{array}$ & & & & $\begin{array}{l}-.1320575-04 \\
-.1203069-04 \\
-.1100574-04 \\
-.1010639-04 \\
-.9129229-05\end{array}$ & $\begin{array}{l}.1832631-06 \\
.1604313-06 \\
.1412686-06 \\
.1250638-06 \\
.1081389-06\end{array}$ \\
\hline $\begin{array}{l}120.000 \\
125.000 \\
130.000\end{array}$ & $\begin{array}{l}.5573990+00 \\
.5546594+00 \\
.5520114+00\end{array}$ & & & & & & & & & & & $\begin{array}{l}.8250771-07 \\
.7271789-07 \\
.6443054-07 \\
.5736688-07\end{array}$ \\
\hline & $\begin{array}{l}.5469695+00 \\
.5445664+00 \\
.5422361+00 \\
.5377790+00\end{array}$ & $\begin{array}{l}.6907292-03 \\
.6640503-03 \\
.6393001-03 \\
.6162786-03 \\
.5747513-03\end{array}$ & $\begin{array}{l}-.8846713-06 \\
-.8221356-06 \\
-.7660337-06 \\
-.7155108-06 \\
-.6284455-06\end{array}$ & & & & & & & & & $\begin{array}{l}.5130812-07 \\
.4608093-07 \\
.4154673-07 \\
.3759384-07 \\
.3108570-07\end{array}$ \\
\hline $\begin{array}{l}180.000 \\
190.000 \\
200.000 \\
210.000\end{array}$ & $\begin{array}{l}.5295863+00 \\
.5258056+00 \\
.5222102+00 \\
.5187839+00\end{array}$ & $\begin{array}{l}.5383297-03 \\
.5061347-03 \\
.4774774-03 \\
.4518102-03 \\
.4286923-03\end{array}$ & $\begin{array}{l}-.5564254-06 \\
-.4961680-06 \\
-.4452403-06 \\
-.4018077-06 \\
-.3644656-06\end{array}$ & $\begin{array}{l}.1931560-08 \\
.1650999-08 \\
.1422774-08 \\
.1235163-08\end{array}$ & $-.7027771-01$ & $-.4613395-03$ & $\begin{array}{l}.1116433-05 \\
.9943847-06 \\
.8913817-06 \\
.8036534-06 \\
.7283188-06\end{array}$ & \begin{tabular}{|}
$-.6628986-08$ \\
$-.5609382-08$ \\
$-.4790497-08$ \\
$-.4125033-08$ \\
$-.3578498-08$
\end{tabular} & $\begin{array}{l}.6478457-01 \\
.6585949-01 \\
.6679182-01 \\
.6760428-01 \\
.6831515-01\end{array}$ & & $\begin{array}{l}-.3379394-05 \\
-.3006928-05 \\
-.2692977-05 \\
-.2425891-05 \\
-.2196778-05\end{array}$ & $\begin{array}{l}.2600937-07 \\
.2199013-07 \\
.1876529-07 \\
.1614701-07 \\
.1399846-07\end{array}$ \\
\hline $\begin{array}{l}240.000 \\
260.000 \\
280.000 \\
300.000\end{array}$ & $\begin{array}{l}.5155126+00 \\
.5093867+00 \\
.5037481+00 \\
.4985294+00 \\
.4936759+00\end{array}$ & $\begin{array}{l}.4077653-03 \\
.3713558-03 \\
.3407695-03 \\
.3147234-03 \\
.2922844-03\end{array}$ & $\begin{array}{l}.2791931-06 \\
.2380360-06 \\
.2053966-06 \\
.1790722-06\end{array}$ & $\begin{array}{l}.1079471-08 \\
.8392094-09 \\
.6658854-09 \\
.5376127-09 \\
.4405843-09\end{array}$ & $\begin{array}{l}-.7035622-01 \\
-.7043741-01 \\
-.7044183-01\end{array}$ & $\begin{array}{l}-.4385560-03 \\
-.3989700-03 \\
-.3657690-03 \\
-.3375364-03 \\
-.3132441-03\end{array}$ & $\begin{array}{l}.6631455-06 \\
.5566400-06 \\
.4739753-06 \\
.4085224-06 \\
.3558074-06\end{array}$ & $\begin{array}{l}-.3125332-08 \\
-.2426798-08 \\
-.1923570-08 \\
-.1551592-08 \\
-.1270519-08\end{array}$ & $\begin{array}{l}.6893929-01 \\
.6997408-01 \\
.7078355-01 \\
.7142046-01 \\
.7192298-01\end{array}$ & $\begin{array}{l}.9157844-03 \\
.8323700-03 \\
.7625050-03 \\
.7031655-03 \\
.6521610-03\end{array}$ & $\begin{array}{l}-.1998762-05 \\
-.1675586-05 \\
-.1425147-05 \\
-.1227127-05 \\
-.1067839-05\end{array}$ & $\begin{array}{l}.1221832-07 \\
.9477108-08 \\
.7504779-08 \\
.6048451-08 \\
.4949085-08\end{array}$ \\
\hline
\end{tabular}




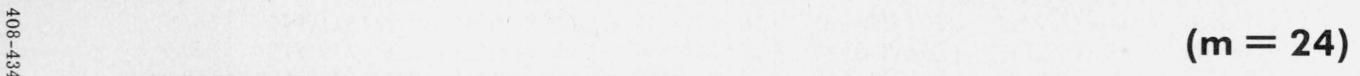

\begin{tabular}{|c|c|c|c|c|c|c|c|c|c|c|c|c|}
\hline $\mathrm{T}^{*}$ & $\mathrm{~B}^{*(0)}$ & $\mathrm{B}^{*(1)}$ & $\mathrm{B}^{*(2)}$ & $\mathrm{B}^{*(3)}$ & $\mathrm{T}^{*} \frac{\partial \mathrm{B}^{*(0)}}{\partial \mathrm{T}^{*}}$ & $\mathrm{~T}^{*} \frac{\partial \mathrm{B}^{*(1)}}{\partial \mathrm{T}^{*}}$ & $T^{*} \frac{\partial B^{*(2)}}{\partial T^{*}}$ & $\mathrm{~T}^{*} \frac{\partial \mathrm{B}^{*(3)}}{\partial \mathrm{T}^{*}}$ & $\mathrm{~T}^{* 2} \frac{\partial^{2} \mathrm{~B}^{*(0)}}{\partial \mathrm{T}^{* 2}}$ & $\mathrm{~T}^{* 2} \frac{\partial^{2} \mathrm{~B}^{*(1)}}{\partial \mathrm{T}^{* 2}}$ & $\mathrm{~T}^{* 2} \frac{\partial^{2} \mathrm{~B}^{*(2)}}{\partial \mathrm{T}^{* 2}}$ & $T^{* 2} \frac{\partial^{2} B^{*(3)}}{\partial T^{* 2}}$ \\
\hline $\begin{array}{l}.300 \\
.400 \\
.500 \\
.600 \\
.700\end{array}$ & $\begin{array}{l}-.1794584+02 \\
-.8799016+01 \\
-.5472878+01 \\
-.3810922+01 \\
-.2825992+01\end{array}$ & $\begin{array}{l}.3594153+02 \\
.1029929+02 \\
.4551343+01 \\
.2530452+01 \\
.1615514+01\end{array}$ & $\begin{array}{l}-.1153001+03 \\
-.2075408+02 \\
-.6458740+01 \\
-.2713016+01 \\
-.1372319+01\end{array}$ & $\begin{array}{l}.5197400+03 \\
.6305114+02 \\
.1464080+02 \\
.4880402+01 \\
.2041055+01\end{array}$ & $\begin{array}{l}.4957408+02 \\
.1976169+02 \\
.1112450+02 \\
.7432031+01 \\
.5476885+01\end{array}$ & $\begin{array}{l}-.1723089+03 \\
-.4050556+02 \\
-.1549468+02 \\
-.7704739+01 \\
-.4496566+01\end{array}$ & $\begin{array}{l}.7421625+03 \\
.1146331+03 \\
.3201639+02 \\
.1239195+02 \\
.5875153+01\end{array}$ & $\begin{array}{l}-.4076358+04 \\
-.4325930+03 \\
-.9142552+02 \\
-.2838833+02 \\
-.1122589+02\end{array}$ & $\begin{array}{l}-.2294472+03 \\
-.7536302+02 \\
-.3736669+02 \\
-.2287233+02 \\
-.1582763+02\end{array}$ & $\begin{array}{l}.1121002+04 \\
.2267450+03 \\
.7797563+02 \\
.3575892+02 \\
.1956713+02\end{array}$ & $\begin{array}{l}-.5934254+04 \\
-.8057004+03 \\
-.2056035+03 \\
-.7435987+02 \\
-.3342053+02\end{array}$ & $\begin{array}{l}.3804531+05 \\
.3591109+04 \\
.6992436+03 \\
.2041624+03 \\
.7691655+02\end{array}$ \\
\hline $\begin{array}{l}.750 \\
.775 \\
.800 \\
.825 \\
.850\end{array}$ & $\begin{array}{l}-.2471435+01 \\
-.2317999+01 \\
-.2177764+01 \\
-.2049114+01 \\
-.1930686+01\end{array}$ & $\begin{array}{l}.1338177+01 \\
.1226669+01 \\
.1129233+01 \\
.1043595+01 \\
.9679191+00\end{array}$ & $\begin{array}{l}-.1025525+01 \\
-.8954227+00 \\
-.7864944+00 \\
-.6946263+00 \\
-.6166207+00\end{array}$ & $\begin{array}{l}.1402877+01 \\
.1177530+01 \\
.9957141+00 \\
.8477511+00 \\
.7263705+00\end{array}$ & $\begin{array}{l}.4819420+01 \\
.4542826+01 \\
.4294285+01 \\
.4069890+01 \\
.3866408+01\end{array}$ & $\begin{array}{l}-.3583055+01 \\
-.3225573+01 \\
-.2918353+01 \\
-.2652616+01 \\
-.2421377+01\end{array}$ & $\begin{array}{l}.4270474+01 \\
.3680916+01 \\
.3193527+01 \\
.2787433+01 \\
.2446608+01\end{array}$ & $\begin{array}{l}-.7533580+01 \\
-.6253514+01 \\
-.5232089+01 \\
-.4409597+01 \\
-.3741678+01\end{array}$ & $\begin{array}{l}-.1358315+02 \\
-.1266025+02 \\
-.1184240+02 \\
-.1111369+02 \\
-.1046115+02\end{array}$ & $\begin{array}{l}.1516603+02 \\
.1347764+02 \\
.1204414+02 \\
.1081851+02 \\
.9763828+01\end{array}$ & $\begin{array}{l}-.2374474+02 \\
-.2025082+02 \\
-.1739242+02 \\
-.1503451+02 \\
-.1307443+02\end{array}$ & $\begin{array}{l}.5056244+02 \\
.4157000+02 \\
.3446271+02 \\
.2879165+02 \\
.2422646+02\end{array}$ \\
\hline $\begin{array}{l}.875 \\
.900 \\
.925 \\
.950 \\
.975\end{array}$ & $\begin{array}{l}-.1821322+01 \\
-.1720030+01 \\
-.1625956+01 \\
-.1538365+01 \\
-.1456614+01\end{array}$ & $\begin{array}{l}.9007118+00 \\
.8407472+00 \\
.7870127+00 \\
.7386661+00 \\
.6950022+00\end{array}$ & $\begin{array}{l}-.5499683+00 \\
-.4926823+00 \\
-.4431767+00 \\
-.4001758+00 \\
-.3626454+00\end{array}$ & $\begin{array}{l}.6260529+00 \\
.5425660+00 \\
.4726342+00 \\
.4137000+00 \\
.3637504+00\end{array}$ & $\begin{array}{l}.3681144+01 \\
.3511834+01 \\
.3356566+01 \\
.3213715+01 \\
.3081889+01\end{array}$ & $\begin{array}{c}-.2219036+01 \\
-.2041063+01 \\
-.1883767+01 \\
-.1744116+01 \\
-.1619608+01\end{array}$ & $\begin{array}{l}.2158624+01 \\
.1913756+01 \\
.1704321+01 \\
.1524207+01 \\
.1368509+01\end{array}$ & $\begin{array}{l}-.3195005+01 \\
-.2744280+01 \\
-.2370111+01 \\
-.2057499+01 \\
-.1794742+01\end{array}$ & $\begin{array}{l}-.9874097+01 \\
-.9343685+01 \\
-.8862532+01 \\
-.8424435+01 \\
-.8024156+01\end{array}$ & $\begin{array}{l}.8850786+01 \\
.8055938+01 \\
.7360375+01 \\
.6748727+01 \\
.6208416+01\end{array}$ & $\begin{array}{l}-.1143341+02 \\
-.1005033+02 \\
-.8877383+01 \\
-.7876854+01 \\
-.7018738+01\end{array}$ & $\begin{array}{l}.2052119+02 \\
.1749072+02 \\
.1499437+02 \\
.1292418+02 \\
.1119657+02\end{array}$ \\
\hline $\begin{array}{l}1.000 \\
1.020 \\
1.040 \\
1.060 \\
1.080\end{array}$ & $\begin{array}{l}-.1380145+01 \\
-.1322440+01 \\
-.1267577+01 \\
-.1215352+01 \\
-.1165581+01\end{array}$ & $\begin{array}{l}.6554278+00 \\
.6263722+00 \\
.5993888+00 \\
.5742814+00 \\
.5508770+00\end{array}$ & $\begin{array}{l}-.3297421+00 \\
-.3062811+00 \\
-.2850378+00 \\
-.2657555+00 \\
-.2482121+00\end{array}$ & $\begin{array}{l}.3211886+00 \\
.2915922+00 \\
.2653672+00 \\
.2420617+00 \\
.2212928+00\end{array}$ & $\begin{array}{l}.2959896+01 \\
.2868682+01 \\
.2782633+01 \\
.2701333+01 \\
.2624410+01\end{array}$ & $\begin{array}{l}-.1508160+01 \\
-.1427225+01 \\
-.1352768+01 \\
-.1284122+01 \\
-.1220704+01\end{array}$ & $\begin{array}{l}.1233266+01 \\
.1137612+01 \\
.1051602+01 \\
.9740592+00 \\
.9039742+00\end{array}$ & $\begin{array}{l}-.1572639+01 \\
-.1419276+01 \\
-.1284199+01 \\
-.1164861+01 \\
-.1059119+01\end{array}$ & $\begin{array}{l}-.7657241+01 \\
-.7385156+01 \\
-.7130282+01 \\
-.6891113+01 \\
-.6666308+01\end{array}$ & $\begin{array}{l}.5729079+01 \\
.5383689+01 \\
.5068069+01 \\
.4778985+01 \\
.4513619+01\end{array}$ & $\begin{array}{l}-.6278991+01 \\
-.5759253+01 \\
-.5294567+01 \\
-.4877943+01 \\
-.4503418+01\end{array}$ & $\begin{array}{l}.9746306+01 \\
.8750930+01 \\
.7878773+01 \\
.7112118+01 \\
.6436126+01\end{array}$ \\
\hline $\begin{array}{l}1.100 \\
1.120 \\
1.140 \\
1.160 \\
1.180\end{array}$ & $\begin{array}{l}-.1118098+01 \\
-.1072750+01 \\
-.1029397+01 \\
-.9879128+00 \\
-.9481795+00\end{array}$ & $\begin{array}{l}.5290219+00 \\
.5085795+00 \\
.4894278+00 \\
.4714579+00 \\
.4545723+00\end{array}$ & $\begin{array}{l}-.2322152+00 \\
-.2175972+00 \\
-.2042119+00 \\
-.1919313+00 \\
-.1806429+00\end{array}$ & $\begin{array}{l}.2027351+00 \\
.1861107+00 \\
.1711817+00 \\
.1577438+00 \\
.1456207+00\end{array}$ & $\begin{array}{l}.2551527+01 \\
.2482382+01 \\
.2416700+01 \\
.2354233+01 \\
.2294755+01\end{array}$ & $\begin{array}{l}-.1162002+01 \\
-.1107562+01 \\
-.1056985+01 \\
-.1009917+01 \\
-.9660413+00\end{array}$ & $\begin{array}{l}.8404776+00 \\
.7828177+00 \\
.7303425+00 \\
.6824850+00 \\
.6387505+00\end{array}$ & $\begin{array}{l}-.9651579+00 \\
-.8814410+00 \\
-.8066581+00 \\
-.7396903+00 \\
-.6795781+00\end{array}$ & $\begin{array}{l}-.6454669+01 \\
-.6255124+01 \\
-.6066708+01 \\
-.5888556+01 \\
-.5719886+01\end{array}$ & \begin{tabular}{l|}
$.4269511+01$ \\
$.4044502+01$ \\
$.3836698+01$ \\
$.3644426+01$ \\
$.3466210+01$
\end{tabular} & $\begin{array}{l}-.4165881+01 \\
-.3860938+01 \\
-.3584800+01 \\
-.3334188+01 \\
-.3106253+01\end{array}$ & $\begin{array}{l}.5838320+01 \\
.5308164+01 \\
.4836731+01 \\
.4416430+01 \\
.4040783+01\end{array}$ \\
\hline $\begin{array}{l}1.200 \\
1.220 \\
1.240 \\
1.260 \\
1.280\end{array}$ & $\begin{array}{l}-.9100902+00 \\
-.8735459+00 \\
-.8384556+00 \\
-.8047353+00 \\
-.7723072+00\end{array}$ & $\begin{array}{l}.4386831+00 \\
.4237114+00 \\
.4095860+00 \\
.3962424+00 \\
.3836224+00\end{array}$ & $\begin{array}{l}-.1702478+00 \\
-.1606587+00 \\
-.1517983+00 \\
-.1435980+00 \\
-.1359970+00\end{array}$ & $\begin{array}{l}.1346601+00 \\
.1247298+00 \\
.1157151+00 \\
.1075157+00 \\
.1000440+00\end{array}$ & $\begin{array}{l}.2238061+01 \\
.2183964+01 \\
.2132293+01 \\
.2082891+01 \\
.2035614+01\end{array}$ & $\begin{array}{l}-.9250771+00 \\
-.8867734+00 \\
-.8509052+00 \\
-.8172707+00 \\
-.7856883+00\end{array}$ & $\begin{array}{l}.5987059+00 \\
.5619715+00 \\
.5282131+00 \\
.4971359+00 \\
.4684795+00\end{array}$ & $\begin{array}{l}-.6254963+00 \\
-.5767330+00 \\
-.5326722+00 \\
-.4927795+00 \\
-.4565898+00\end{array}$ & $\begin{array}{l}-.5559990+01 \\
-.5408229+01 \\
-.5264021+01 \\
-.5126838+01 \\
-.4996196+01\end{array}$ & \begin{tabular}{l|}
$.3300740+01$ \\
$.3146853+01$ \\
$.3003514+01$ \\
$.2869796+01$ \\
$.2744874+01$
\end{tabular} & $\begin{array}{l}-.2898518+01 \\
-.2708818+01 \\
-.2535258+01 \\
-.2376174+01 \\
-.2230103+01\end{array}$ & $\begin{array}{l}.3704243+01 \\
.3402046+01 \\
.3130089+01 \\
.2884824+01 \\
.2663179+01\end{array}$ \\
\hline $\begin{array}{l}1.300 \\
1.320 \\
1.340 \\
1.360 \\
1.380\end{array}$ & $\begin{array}{l}-.7410993+00 \\
-.7110448+00 \\
-.6820818+00 \\
-.6541526+00 \\
-.6272036+00\end{array}$ & $\begin{array}{l}.3716729+00 \\
.3603457+00 \\
.3495970+00 \\
.3393868+00 \\
.3296785+00\end{array}$ & $\begin{array}{l}-.1289409+00 \\
-.1223814+00 \\
-.1162748+00 \\
-.1105825+00 \\
-.1052694+00\end{array}$ & $\begin{array}{l}.9322332-01 \\
.8698612-01 \\
.8127300-01 \\
.7603153-01 \\
.7121529-01\end{array}$ & $\begin{array}{l}.1990330+01 \\
.1946919+01 \\
.1905267+01 \\
.1865273+01 \\
.1826839+01\end{array}$ & $\begin{array}{l}-.7559944+00 \\
-.7280412+00 \\
-.7016950+00 \\
-.6768347+00 \\
-.6533501+00\end{array}$ & $\begin{array}{l}.4420128+00 \\
.4175307+00 \\
.3948508+00 \\
.3738102+00 \\
.3542631+00\end{array}$ & $\begin{array}{l}-.4236973+00 \\
-.3937473+00 \\
-.3664286+00 \\
-.3414677+00 \\
-.3186238+00\end{array}$ & $\begin{array}{l}-.4871656+01 \\
-.4752816+01 \\
-.4639306+01 \\
-.4530788+01 \\
-.4426950+01\end{array}$ & $\begin{array}{l}.2628005+01 \\
.2518521+01 \\
.2415825+01 \\
.2319372+01 \\
.2228676+01\end{array}$ & $\begin{array}{l}-.2095752+01 \\
-.1971980+01 \\
-.1857773+01 \\
-.1752232+01 \\
-.1654556+01\end{array}$ & $\begin{array}{l}.2462484+01 \\
.2280413+01 \\
.2114934+01 \\
.1964269+01 \\
.1826857+01\end{array}$ \\
\hline $\begin{array}{l}1.400 \\
1.420 \\
1.440 \\
1.460 \\
1.480\end{array}$ & $\begin{array}{l}-.6011847+00 \\
-.5760492+00 \\
-.5517535+00 \\
-.5282567+00 \\
-.5055207+00\end{array}$ & $\begin{array}{l}.3204384+00 \\
.3116360+00 \\
.3032430+00 \\
.2952335+00 \\
.2875835+00\end{array}$ & $\begin{array}{l}-.1003040+00 \\
-.9565813-01 \\
-.9130602-01 \\
-.8722452-01 \\
-.8339263-01\end{array}$ & $\begin{array}{l}.6678315-01 \\
.6269855-01 \\
.5892895-01 \\
.5544534-01 \\
.5222177-01\end{array}$ & $\begin{array}{l}.1789879+01 \\
.1754309+01 \\
.1720055+01 \\
.1687045+01 \\
.1655213+01\end{array}$ & $\begin{array}{l}-.6311413+00 \\
-.6101168+00 \\
-.5901936+00 \\
-.5712955+00 \\
-.5533529+00\end{array}$ & $\begin{array}{l}.3360794+00 \\
.3191419+00 \\
.3033454+00 \\
.2885952+00 \\
.2748058+00\end{array}$ & $\begin{array}{l}-.2976843+00 \\
-.2784609+00 \\
-.2607869+00 \\
-.2445140+00 \\
-.2295105+00\end{array}$ & $\begin{array}{l}-.4327506+01 \\
-.4232192+01 \\
-.4140764+01 \\
-.4052996+01 \\
-.3968679+01\end{array}$ & $\begin{array}{l}.2143291+01 \\
.2062817+01 \\
.1986888+01 \\
.1915171+01 \\
.1847365+01\end{array}$ & $\begin{array}{l}-.1564032+01 \\
-.1480019+01 \\
-.1401947+01 \\
-.1329302+01 \\
-.1261623+01\end{array}$ & $\begin{array}{l}.1701324+01 \\
.1586461+01 \\
.1481197+01 \\
.1384586+01 \\
.1295788+01\end{array}$ \\
\hline $\begin{array}{l}1.500 \\
1.540 \\
1.580 \\
1.620 \\
1.660\end{array}$ & $\begin{array}{l}-.4835097+00 \\
-.4415299+00 \\
-.4020719+00 \\
-.3649185+00 \\
-.3298765+00\end{array}$ & $\begin{array}{l}.2802709+00 \\
.2665785+00 \\
.2540109+00 \\
.2424433+00 \\
.2317680+00\end{array}$ & $\begin{array}{l}-.7979128-01 \\
-.7321237-01 \\
-.6736673-01 \\
-.6215345-01 \\
-.5748790-01\end{array}$ & $\begin{array}{l}.4923503-01 \\
.4389087-01 \\
.3927027-01 \\
.3525742-01 \\
.3175772-01\end{array}$ & $\begin{array}{l}.1624499+01 \\
.1566198+01 \\
.1511728+01 \\
.1460727+01 \\
.1412878+01\end{array}$ & $\begin{array}{l}-.5363019+00 \\
-.5046447+00 \\
-.4759091+00 \\
-.4497426+00 \\
-.4258441+00\end{array}$ & $\begin{array}{l}.2619000+00 \\
.2384658+00 \\
.2178075+00 \\
.1995238+00 \\
.1832812+00\end{array}$ & $\begin{array}{l}-.2156587+00 \\
-.1910008+00 \\
-.1698245+00 \\
-.1515525+00 \\
-.1357165+00\end{array}$ & $\begin{array}{l}-.3887619+01 \\
-.3734566+01 \\
-.3592544+01 \\
-.3460432+01 \\
-.3337252+01\end{array}$ & $\begin{array}{l}.1783193+01 \\
.1664758+01 \\
.1558088+01 \\
.1461684+01 \\
.1374275+01\end{array}$ & $\begin{array}{l}-.1198494+01 \\
-.1084427+01 \\
-.9845133+00 \\
-.8966298+00 \\
-.8190216+00\end{array}$ & $\begin{array}{l}.1214056+01 \\
.1069205+01 \\
.9455222+00 \\
.8393956+00 \\
.7479107+00\end{array}$ \\
\hline $\begin{array}{l}1.700 \\
1.740 \\
1.780 \\
1.820 \\
1.860\end{array}$ & $\begin{array}{l}-.2967738+00 \\
-.2654563+00 \\
-.2357858+00 \\
-.2076379+00 \\
-.1809006+00\end{array}$ & $\begin{array}{l}.2218917+00 \\
.2127331+00 \\
.2042211+00 \\
.1962935+00 \\
.1888955+00\end{array}$ & $\begin{array}{l}-.5329882-01 \\
-.4952584-01 \\
-.4611761-01 \\
-.4303028-01 \\
-.4022622-01\end{array}$ & $\begin{array}{l}.2869339-01 \\
.2600018-01 \\
.2362470-01 \\
.2152238-01 \\
.1965581-01\end{array}$ & $\begin{array}{l}.1367898+01 \\
.1325541+01 \\
.1285584+01 \\
.1247830+01 \\
.1212105+01\end{array}$ & $\begin{array}{l}-.4039554+00 \\
-.3838540+00 \\
-.3653476+00 \\
-.3482688+00 \\
-.3324719+00\end{array}$ & $\begin{array}{l}.1688008+00 \\
.1558482+00 \\
.1442255+00 \\
.1337650+00 \\
.1243235+00\end{array}$ & $\begin{array}{l}-.1219341+00 \\
-.1098915+00 \\
-.9932944-01 \\
-.9003286-01 \\
-.8182243-01\end{array}$ & $\begin{array}{l}-.3222148+01 \\
-.3114370+01 \\
-.3013254+01 \\
-.2918214+01 \\
-.2828729+01\end{array}$ & $\begin{array}{l}.1294781+01 \\
.1222275+01 \\
.1155963+01 \\
.1095159+01 \\
.1039268+01\end{array}$ & $\begin{array}{l}-.7502313+00 \\
-.6890414+00 \\
-.6344300+00 \\
-.5855352+00 \\
-.5416269+00\end{array}$ & $\begin{array}{l}.6687020+00 \\
.5998380+00 \\
.5397326+00 \\
.4870765+00 \\
.4407833+00\end{array}$ \\
\hline
\end{tabular}


$(m=24)$

\begin{tabular}{|c|c|c|c|c|c|c|c|c|c|c|c|c|}
\hline$T^{*}$ & (0) & (1) & $\mathrm{B}^{*(2)}$ & $\mathrm{B}^{*(3)}$ & $\mathrm{T}^{*} \frac{\partial \mathrm{B}^{*(0)}}{\partial \mathrm{T}^{*}}$ & $\mathrm{~T}^{*} \frac{\partial \mathrm{B}^{*(1)}}{\partial \mathrm{T}^{*}}$ & $\mathrm{~T}^{*} \frac{\partial \mathrm{B}^{*(2)}}{\partial \mathrm{T}^{*}}$ & $\mathrm{~T}^{*} \frac{\partial \mathrm{B}^{*(3)}}{\partial \mathrm{T}^{*}}$ & $\mathrm{~T}^{* 2} \frac{\partial^{2} \mathrm{~B}^{*(0)}}{\partial \mathrm{T}^{* 2}}$ & $T^{* 2} \frac{\partial^{2} B^{*(1)}}{\partial T^{* 2}}$ & $T^{* 2} \frac{\partial^{2} B^{*(2)}}{\partial T^{* 2}}$ & $\mathrm{~T}^{* 2} \frac{\partial^{2} \mathrm{~B}^{*(3)}}{\partial \mathrm{T}^{* 2}}$ \\
\hline $\begin{array}{l}1.900 \\
1.940 \\
1.980 \\
2.020 \\
2.060\end{array}$ & $\begin{array}{l}-.1554722+00 \\
-.1312609+00 \\
-.1081830+00 \\
-.8616238-01 \\
-.6512949-01\end{array}$ & $\begin{array}{l}.1819785+00 \\
.1754997+00 \\
.1694210+00 \\
.1637083+00 \\
.1583314+00\end{array}$ & $\begin{array}{l}-.3767301-01 \\
-.3534264-01 \\
-.3321080-01 \\
-.3125633-01 \\
-.2946073-01\end{array}$ & $\begin{array}{l}.1799350-01 \\
.1650878-01 \\
.1517901-01 \\
.1398485-01 \\
.1290977-01\end{array}$ & $\begin{array}{l}.1178248+01 \\
.1146119+01 \\
.1115589+01 \\
.1086543+01 \\
.1058874+01\end{array}$ & $\begin{array}{l}-.3178292+00 \\
-.3042287+00 \\
-.2915716+00 \\
-.2797706+00 \\
-.2687485+00\end{array}$ & $\begin{array}{l}.1157788+00 \\
.1080256+00 \\
.1009735+00 \\
.9454392-01 \\
.8866890-01\end{array}$ & $\begin{array}{l}-.7454788-01 \\
-.6808276-01 \\
-.6232022-01 \\
-.5716958-01 \\
-.5255359-01\end{array}$ & $\begin{array}{l}-.2744335+01 \\
-.2664617+01 \\
-.2589204+01 \\
-.2517763+01 \\
-.2449993+01\end{array}$ & \begin{tabular}{l|}
$.9877748+00$ \\
$.9402273+00$ \\
$.8962310+00$ \\
$.8554389+00$ \\
$.8175453+00$
\end{tabular} & $\begin{array}{l}-.5020836+00 \\
-.4663745+00 \\
-.4340442+00 \\
-.4047005+00 \\
-.3780048+00\end{array}$ & $\begin{array}{l}.3999474+00 \\
.3638100+00 \\
.3317330+00 \\
.3031774+00 \\
.2776859+00\end{array}$ \\
\hline $\begin{array}{l}2.100 \\
2.140 \\
2.180 \\
2.220 \\
2.260\end{array}$ & $\begin{array}{r}-.4502079-01 \\
-.2577801-01 \\
-.7347686-02 \\
.1031938-01 \\
.2726850-01\end{array}$ & $\begin{array}{l}.1532629+00 \\
.1484785+00 \\
.1439560+00 \\
.1396757+00 \\
.1356195+00\end{array}$ & $\begin{array}{l}.2780782-01 \\
.2628337-01 \\
.2487482-01 \\
.2357111-01 \\
.2236240-01\end{array}$ & $\begin{array}{l}.1193955-01 \\
.1106193-01 \\
.1026632-01 \\
.9543528-02 \\
.8885545-02\end{array}$ & $\begin{array}{l}.1032488+01 \\
.1007298+01 \\
.9832255+00 \\
.9601968+00 \\
.9381462+00\end{array}$ & $\begin{array}{l}-.2584365+00 \\
-.2487733+00 \\
-.2397041+00 \\
-.2311799+00 \\
-.2231566+00\end{array}$ & $\begin{array}{l}.8328913-01 \\
.7835280-01 \\
.7381450-01 \\
.6963432-01 \\
.6577708-01\end{array}$ & $\begin{array}{l}-.4840624-01 \\
-.4467086-01 \\
-.4129867-01 \\
-.3824757-01 \\
-.3548105-01\end{array}$ & $\begin{array}{l}-.2385622+01 \\
-.2324405+01 \\
-.2266119+01 \\
-.2210561+01 \\
-.2157547+01\end{array}$ & \begin{tabular}{l|}
$.7822800+00$ \\
$.7494033+00$ \\
$.7187023+00$ \\
$.6899872+00$ \\
$.6630884+00$
\end{tabular} & $\begin{array}{l}-.3536632+00 \\
-.3314204+00 \\
-.3110534+00 \\
-.2923672+00 \\
-.2751906+00\end{array}$ & $\begin{array}{l}.2548693+00 \\
.2343950+00 \\
.2159779+00 \\
.1993726+00 \\
.1843675+00\end{array}$ \\
\hline $\begin{array}{l}2.300 \\
2.340 \\
2.380 \\
2.420 \\
2.460\end{array}$ & $\begin{array}{l}.4354148-01 \\
.5917698-01 \\
.7421075-01 \\
.8867597-01 \\
.1026034+00\end{array}$ & $\begin{array}{l}.1317712+00 \\
.1281159+00 \\
.1246401+00 \\
.1213316+00 \\
.1181792+00\end{array}$ & & & $\begin{array}{l}.9170127+00 \\
.8967406+00 \\
.8772784+00 \\
.8585784+00 \\
.8405970+00\end{array}$ & & & & & & $\begin{array}{l}-.2593728+00 \\
-.2447810+00 \\
-.2312975+00 \\
-.2188181+00 \\
-.2072500+00\end{array}$ & $\begin{array}{l}.1707792+00 \\
.1584485+00 \\
.1472369+00 \\
.1370234+00 \\
.1277020+00\end{array}$ \\
\hline $\begin{array}{l}2.500 \\
2.600 \\
2.700 \\
2.800 \\
2.900\end{array}$ & $\begin{array}{l}.1160216+00 \\
.1475099+00 \\
.1763468+00 \\
.2028440+00 \\
.2272669+00\end{array}$ & & $\begin{array}{l}-.1667746-01 \\
-.1490829-01 \\
-.1339585-01 \\
-.1209397-01 \\
-.1096625-01\end{array}$ & $\begin{array}{l}.5962254-02 \\
.5116683-02 \\
.4421160-02 \\
.3844232-02 \\
.3361961-02\end{array}$ & $\begin{array}{l}.8232936+00 \\
.7827610+00 \\
.7457208+00 \\
.7117404+00 \\
.6804560+00\end{array}$ & & $\begin{array}{l}.4789889-01 \\
.4243603-01 \\
.3780996-01 \\
.3386359-01 \\
.3047418-01\end{array}$ & & & & $\begin{array}{l}-.1965106+00 \\
-.1728230+00 \\
-.1529172+00 \\
-.1360590+00 \\
-.1216797+00\end{array}$ & $\begin{array}{l}.1191797+00 \\
.1008572+00 \\
.8600552-01 \\
.7385609-01 \\
.6383278-01\end{array}$ \\
\hline & $\begin{array}{l}.2498420+00 \\
.2707644+00 \\
.2902030+00 \\
.3083045+00 \\
.3251969+00\end{array}$ & $\begin{array}{l}.8261171-01 \\
.7878854-01 \\
.7528002-01 \\
.7205018-01\end{array}$ & & & $\begin{array}{l}.6515589+00 \\
.6247859+00 \\
.5999110+00 \\
.5767391+00 \\
.5551010+00\end{array}$ & $\begin{array}{l}-.1312316+00 \\
-.1238135+00 \\
-.1170998+00 \\
-.1110004+00 \\
-.1054392+00\end{array}$ & $\begin{array}{l}.2754491-01 \\
.2499866-01 \\
.2277347-01 \\
.2081916-01 \\
.1909476-01\end{array}$ & & & & & $\begin{array}{l}.5549876-01 \\
.4851907-01 \\
.4263433-01 \\
.3764173-01 \\
.3338134-01\end{array}$ \\
\hline & $\begin{array}{l}.3409926+00 \\
.3557906+00 \\
.3696786+00 \\
.3827341+00 \\
.3950263+00\end{array}$ & $\begin{array}{l}.6630759-01 \\
.6374535-01 \\
.6136155-01 \\
.5913877-01\end{array}$ & $\begin{array}{l}-.6568574-02 \\
-.6093135-02 \\
-.5665951-02 \\
-.5280840-02 \\
-.4932564-02\end{array}$ & & & & $\begin{array}{l}.1756663-01 \\
.1620692-01 \\
.1499246-01 \\
.1390385-01 \\
.1292476-01\end{array}$ & & & $\begin{array}{l}.2707830+00 \\
.2567901+00 \\
.2439909+00 \\
.2322489+00 \\
.2214468+00\end{array}$ & $\begin{array}{l}-.6795622-01 \\
-.6241519-01 \\
-.5748989-01 \\
-.5309540-01 \\
-.4916061-01\end{array}$ & $\begin{array}{l}.2972596-01 \\
.2657368-01 \\
.2384225-01 \\
.2146484-01 \\
.1938681-01\end{array}$ \\
\hline $\begin{array}{l}4.200 \\
4.300 \\
4.400\end{array}$ & $\begin{array}{l}.4066171+00 \\
.4175618+00 \\
.4279101+00 \\
.4377069+00 \\
.4469927+00\end{array}$ & $\begin{array}{l}.5706173-01 \\
.5511700-01 \\
.5329271-01 \\
.5157834-01 \\
.4996454-01\end{array}$ & $\begin{array}{l}-.4616661-02 \\
-.4329320-02 \\
-.4067262-02 \\
-.3827663-02 \\
-.3608074-02\end{array}$ & $\begin{array}{l}.9336604-03 \\
.8562032-03 \\
.7870028-03 \\
.7249963-03\end{array}$ & $\begin{array}{l}.4503597+00 \\
.4361843+00 \\
.4227501+00 \\
.4100001+00 \\
.3978835+00\end{array}$ & $\begin{array}{l}-.8035139-01 \\
-.7718879-01 \\
-.7424262-01 \\
-.7149248-01 \\
-.6892037-01\end{array}$ & $\begin{array}{l}.1204137-01 \\
.1124193-01 \\
.1051643-01 \\
.9856237-02 \\
.9253956-02\end{array}$ & & $\begin{array}{l}-.1033017+01 \\
-.1001774+01 \\
-.9722418+00 \\
-.9442840+00 \\
-.9177777+00\end{array}$ & $\begin{array}{l}.2114831+00 \\
.2022700+00 \\
.1937312+00 \\
.1857997+00 \\
.1784169+00\end{array}$ & $\begin{array}{l}-.4562567-01 \\
-.4243991-01 \\
-.3956030-01 \\
-.3695005-01 \\
-.3457764-01\end{array}$ & $\begin{array}{l}.1756320-01 \\
.1595683-01 \\
.1453677-01 \\
.1327717-01 \\
.1215631-01\end{array}$ \\
\hline $\begin{array}{l}4.500 \\
4.600 \\
4.700 \\
4.800 \\
4.900\end{array}$ & $\begin{array}{l}.4558042+00 \\
.4641746+00 \\
.4721342+00 \\
.4797107+00 \\
.4869293+00\end{array}$ & $\begin{array}{l}.4844297-01 \\
.4700617-01 \\
.4564745-01 \\
.4436079-01 \\
.4314077-01\end{array}$ & $\begin{array}{l}-.3049412-02 \\
-.2891112-02 \\
-.2744532-02\end{array}$ & & $\begin{array}{l}.3863540+00 \\
.3753698+00 \\
.3648932+00 \\
.3548897+00 \\
.3453278+00\end{array}$ & $\begin{array}{l}-.6651037-01 \\
-.6424834-01 \\
-.6212168-01 \\
-.6011915-01 \\
-.5823069-01\end{array}$ & $\begin{array}{l}.8703175-02 \\
.8198330-02 \\
.7734578-02 \\
.7307686-02 \\
.6913940-02\end{array}$ & $\begin{array}{l}-.2377737-02 \\
-.2192726-02 \\
-.2026173-02 \\
-.1875857-02 \\
-.1739866-02\end{array}$ & $\begin{array}{l}-.8926129+00 \\
-.8686903+00 \\
-.8459201+00 \\
-.8242209+00 \\
-.8035189+00\end{array}$ & $\begin{array}{l}.1715313+00 \\
.1650972+00 \\
.1590742+00 \\
.1534266+00 \\
.1481222+00\end{array}$ & $\begin{array}{l}-.3241591-01 \\
-.3044140-01 \\
-.2863374-01 \\
-.2697521-01 \\
-.2545033-01\end{array}$ & $\begin{array}{l}.1115587-01 \\
.1026034-01 \\
.9456492-02 \\
.8733054-02 \\
.8080347-02\end{array}$ \\
\hline $\begin{array}{l}5.000 \\
5.100 \\
5.200 \\
5.300 \\
5.400\end{array}$ & $\begin{array}{l}.4938131+00 \\
.5003833+00 \\
.5066592+00 \\
.5126589+00 \\
.5183988+00\end{array}$ & $\begin{array}{l}.4198247-01 \\
.4088147-01 \\
.3983371-01 \\
.3883554-01 \\
.3788360-01\end{array}$ & $\begin{array}{l}-.2482221-02 \\
-.2364633-02 \\
-.2255022-02 \\
-.2152693-02\end{array}$ & $\begin{array}{l}.4615385-03 \\
.4306499-03 \\
.4024387-03 \\
.3766228-03 \\
.3529546-03\end{array}$ & $\begin{array}{l}.3361789+00 \\
.3274167+00 \\
.3190172+00 \\
.3109582+00 \\
.3032195+00\end{array}$ & $\begin{array}{l}-.5644726-01 \\
-.5476071-01 \\
-.5316371-01 \\
-.5164961-01 \\
-.5021241-01\end{array}$ & $\begin{array}{l}.6550078-02 \\
.6213221-02 \\
.5900823-02 \\
.5610625-02 \\
.5340620-02\end{array}$ & $\begin{array}{l}-.1616551-02 \\
-.1504483-02 \\
-.1402421-02 \\
-.1309284-02 \\
-.1224125-02\end{array}$ & $\begin{array}{l}-.7837469+00 \\
-.7648435+00 \\
-.7467527+00 \\
-.7294230+00 \\
-.7128075+00\end{array}$ & $\begin{array}{l}.1431325+00 \\
.1384320+00 \\
.1339975+00 \\
.1298084+00 \\
.1258460+00\end{array}$ & $\begin{array}{l}-.2404553-01 \\
-.2274889-01 \\
-.2154990-01 \\
-.2043928-01 \\
-.1940878-01\end{array}$ & $\begin{array}{l}.7490045-02 \\
.6954958-02 \\
.6468860-02 \\
.6026339-02 \\
.5622677-02\end{array}$ \\
\hline $\begin{array}{l}5.500 \\
5.600 \\
5.800 \\
6.000 \\
6.200\end{array}$ & $\begin{array}{l}.5238941+00 \\
.5291590+00 \\
.5390486+00 \\
.5481612+00 \\
.5565774+00\end{array}$ & $\begin{array}{l}.3697485-01 \\
.3610647-01 \\
.3448081-01 \\
.3298849-01 \\
.3161412-01\end{array}$ & $\begin{array}{l}-.1967462-02 \\
-.1804694-02 \\
-.1660948-02 \\
-.1533410-02\end{array}$ & $\begin{array}{l}.3312169-03 \\
.3112177-03 \\
.2757769-03 \\
.2454940-03 \\
.2194681-03\end{array}$ & $\begin{array}{l}.2957823+00 \\
.2886293+00 \\
.2751128+00 \\
.2625555+00 \\
.2508587+00\end{array}$ & $\begin{array}{l}-.4884663-01 \\
-.4754731-01 \\
-.4513028-01 \\
-.4292952-01 \\
-.4091831-01\end{array}$ & $\begin{array}{l}.5089019-02 \\
.4854223-02 \\
.4429466-02 \\
.4056557-02 \\
.3727547-02\end{array}$ & $\begin{array}{l}-.1146116-02 \\
-.1074529-02 \\
-.9481279-03 \\
-.8406289-03 \\
-.7486521-03\end{array}$ & $\begin{array}{l}-.6968627+00 \\
-.6815488+00 \\
-.6526694+00 \\
-.6259071+00 \\
-.6010369+00\end{array}$ & $\begin{array}{l}.1220934+00 \\
.1185352+00 \\
.1119478+00 \\
.1059866+00 \\
.1005706+00\end{array}$ & $\begin{array}{l}-.1845109-01 \\
-.1755971-01 \\
-.1595318-01 \\
-.1454956-01 \\
-.1331687-01\end{array}$ & $\begin{array}{l}.5253750-02 \\
.4915947-02 \\
.4321378-02 \\
.3817792-02 \\
.3388598-02\end{array}$ \\
\hline
\end{tabular}


$(m=24)$

\begin{tabular}{|c|c|c|c|c|c|c|c|c|c|c|c|c|}
\hline $\mathrm{T}^{*}$ & $\mathrm{~B}^{*(0)}$ & $\mathrm{B}^{*(1)}$ & $\mathrm{B}^{*(2)}$ & $\mathrm{B}^{*(3)}$ & $\mathrm{T}^{*} \frac{\partial \mathrm{B}^{*(0)}}{\partial \mathrm{T}^{*}}$ & $\mathrm{~T}^{*} \frac{\partial \mathrm{B}^{*(1)}}{\partial \mathrm{T}^{*}}$ & $\mathrm{~T}^{*} \frac{\partial \mathrm{B}^{*(2)}}{\partial \mathrm{T}^{*}}$ & $\mathrm{~T}^{*} \frac{\partial \mathrm{B}^{*(3)}}{\partial \mathrm{T}^{*}}$ & $\mathrm{~T}^{* 2} \frac{\partial^{2} \mathrm{~B}^{*(0)}}{\partial \mathrm{T}^{* 2}}$ & $T^{* 2} \frac{\partial^{2} B^{*(1)}}{\partial T^{* 2}}$ & $\mathrm{~T}^{* 2} \frac{\partial^{2} \mathrm{~B}^{*(2)}}{\partial \mathrm{T}^{* 2}}$ & $\mathrm{~T}^{* 2} \frac{\partial^{2} \mathrm{~B}^{*(3)}}{\partial \mathrm{T}^{* 2}}$ \\
\hline $\begin{array}{l}6.400 \\
6.600 \\
6.800 \\
7.000 \\
7.200\end{array}$ & $\begin{array}{l}.5643674+00 \\
.5715925+00 \\
.5783063+00 \\
.5845560+00 \\
.5903833+00\end{array}$ & \begin{tabular}{l|}
$.3034455-01$ \\
$.2916850-01$ \\
$.2807622-01$ \\
$.2705927-01$ \\
$.2611026-01$
\end{tabular} & $\begin{array}{l}-.1419761-02 \\
-.1318081-02 \\
-.1226767-02 \\
-.1144472-02 \\
-.1070060-02\end{array}$ & $\begin{array}{l}.1969799-03 \\
.1774506-03 \\
.1604111-03 \\
.1454783-03 \\
.1323377-03\end{array}$ & $\begin{array}{l}.2399368+00 \\
.2297150+00 \\
.2201281+00 \\
.2111187+00 \\
.2026359+00\end{array}$ & $\begin{array}{l}-.3907406-01 \\
-.3737754-01 \\
-.3581231-01 \\
-.3436422-01 \\
-.3302103-01\end{array}$ & $\begin{array}{l}.3435932-02 \\
.3176355-02 \\
.2944372-02 \\
.2736275-02 \\
.2548947-02\end{array}$ & $\begin{array}{l}-.6695134-03 \\
-.6010640-03 \\
-.5415700-03 \\
-.4896225-03 \\
-.4440693-03\end{array}$ & $\begin{array}{l}-.5778646+00 \\
-.5562216+00 \\
-.5359611+00 \\
-.5169543+00 \\
-.4990880+00\end{array}$ & $\begin{array}{l}.9563187-01 \\
.9111278-01 \\
.8696449-01 \\
.8314522-01 \\
.7961903-01\end{array}$ & $\begin{array}{l}-.1222907-01 \\
-.1126484-01 \\
-.1040655-01 \\
-.9639573-02 \\
-.8951681-02\end{array}$ & $\begin{array}{l}.3020678-02 \\
.2703575-02 \\
.2428886-02 \\
.2189810-02 \\
.1980807-02\end{array}$ \\
\hline $\begin{array}{l}7.400 \\
7.600 \\
7.800 \\
8.000 \\
8.200\end{array}$ & $\begin{array}{l}.5958251+00 \\
.6009144+00 \\
.6056805+00 \\
.6101495+00 \\
.6143451+00\end{array}$ & $\begin{array}{l}.2522276-01 \\
.2439108-01 \\
.2361023-01 \\
.2287578-01 \\
.2218378-01\end{array}$ & $\begin{array}{l}-.1002568-02 \\
-.9411713-03 \\
-.8851665-03 \\
-.8339467-03 \\
-.7869873-03\end{array}$ & $\begin{array}{l}.1207290-03 \\
.1104362-03 \\
.1012785-03 \\
.9310411-04 \\
.8578481-04\end{array}$ & $\begin{array}{l}.1946350+00 \\
.1870759+00 \\
.1799231+00 \\
.1731446+00 \\
.1667117+00\end{array}$ & $\begin{array}{l}-.3177214-01 \\
-.3060827-01 \\
-.2952131-01 \\
-.2850412-01 \\
-.2755041-01\end{array}$ & $\begin{array}{l}.2379762-02 \\
.2226488-02 \\
.2087223-02 \\
.1960336-02 \\
.1844427-02\end{array}$ & $\begin{array}{l}-.4039616-03 \\
-.3685140-03 \\
-.3370726-03 \\
-.3090900-03 \\
-.2841056-03\end{array}$ & $\begin{array}{l}-.4822624+00 \\
-.4663888+00 \\
-.4513884+00 \\
-.4371909+00 \\
-.4237333+00\end{array}$ & $\begin{array}{l}.7635492-01 \\
.7332596-01 \\
.7050871-01 \\
.6788265-01 \\
.6542976-01\end{array}$ & $\begin{array}{l}-.8332593-02 \\
-.7773620-02 \\
-.7267383-02 \\
-.6807585-02 \\
-.6388827-02\end{array}$ & $\begin{array}{l}.1797330-02 \\
.1635628-02 \\
.1492589-02 \\
.1365617-02 \\
.1252533-02\end{array}$ \\
\hline $\begin{array}{l}8.400 \\
8.600 \\
8.800 \\
9.000 \\
9.200\end{array}$ & $\begin{array}{l}.6182885+00 \\
.6219987+00 \\
.6254931+00 \\
.6287874+00 \\
.6318958+00\end{array}$ & \begin{tabular}{l|}
$.2153075-01$ \\
$.2091353-01$ \\
$.2032931-01$ \\
$.1977558-01$ \\
$.1925003-01$
\end{tabular} & $\begin{array}{l}-.7438334-03 \\
-.7040886-03 \\
-.6674066-03 \\
-.6334835-03 \\
-.6020520-03\end{array}$ & & $\begin{array}{l}.1605988+00 \\
.1547824+00 \\
.1492417+00 \\
.1439573+00 \\
.1389119+00\end{array}$ & $\begin{array}{l}-.2665460-01 \\
-.2581173-01 \\
-.2501739-01 \\
-.2426763-01 \\
-.2355891-01\end{array}$ & $\begin{array}{l}.1738283-02 \\
.1640854-02 \\
.1551226-02 \\
.1468599-02 \\
.1392273-02\end{array}$ & $\begin{array}{l}-.2617304-03 \\
-.2416341-03 \\
-.2235351-03 \\
-.2071923-03 \\
-.1923988-03\end{array}$ & $\begin{array}{l}-.4109591+00 \\
-.3988173+00 \\
-.3872620+00 \\
-.3762516+00 \\
-.3657482+00\end{array}$ & $\begin{array}{l}.6313416-01 \\
.6098180-01 \\
.5896021-01 \\
.5705830-01 \\
.5526615-01\end{array}$ & $\begin{array}{l}-.6006461-02 \\
-.5656471-02 \\
-.5335371-02 \\
-.5040127-02 \\
-.4768085-02\end{array}$ & $\begin{array}{l}.1151502-02 \\
.1060971-02 \\
.9796202-03 \\
.9063214-03 \\
.8401089-03\end{array}$ \\
\hline $\begin{array}{r}9.400 \\
9.600 \\
9.800 \\
10.000 \\
10.200\end{array}$ & & & & & $\begin{array}{l}.1340898+00 \\
.1294763+00 \\
.1250584+00 \\
.1208238+00 \\
.1167613+00\end{array}$ & & $\begin{array}{l}.1321632-02 \\
.1256133-02 \\
.1195296-02 \\
.1138693-02 \\
.1085948-02\end{array}$ & & $\begin{array}{l}-.3557176+00 \\
-.3461285+00 \\
-.3369521+00 \\
-.3281625+00 \\
-.3197354+00\end{array}$ & $\begin{array}{l}.5357489-01 \\
.5197657-01 \\
.5046400-01 \\
.4903074-01 \\
.4767092-01\end{array}$ & $\begin{array}{l}-.4516920-02 \\
-.4284588-02 \\
-.4069285-02 \\
-.3869415-02 \\
-.3683563-02\end{array}$ & $\begin{array}{l}.7801517-03 \\
.7257326-03 \\
.6762302-03 \\
.6311045-03 \\
.5898850-03\end{array}$ \\
\hline $\begin{array}{l}10.400 \\
10.600 \\
10.800 \\
11.000 \\
11.400\end{array}$ & $\begin{array}{l}.6472946+00 \\
.6494086+00 \\
.6514143+00 \\
.6533184+00 \\
.6568451+00\end{array}$ & & $\begin{array}{l}-.4542797-03 \\
-.4349738-03 \\
-.4168610-03 \\
-.3998457-03 \\
-.3687711-03\end{array}$ & & $\begin{array}{l}.1128607+00 \\
.1091125+00 \\
.1055081+00 \\
.1020392+00 \\
.9547883-01\end{array}$ & $\begin{array}{l}-.2001033-01 \\
-.1951507-01 \\
-.1904243-01 \\
-.1859093-01 \\
-.1774608-01\end{array}$ & & & $\begin{array}{l}-.3116490+00 \\
-.3038829+00 \\
-.2964184+00 \\
-.2892381+00 \\
-.2756678+00\end{array}$ & & $\begin{array}{l}-.3510470-02 \\
-.3349013-02 \\
-.3198189-02 \\
-.3057100-02 \\
-.2800980-02\end{array}$ & $\begin{array}{l}.5521601-03 \\
.5175691-03 \\
.4857947-03 \\
.4565574-03 \\
.4047337-03\end{array}$ \\
\hline $\begin{array}{l}11.800 \\
12.200 \\
12.600 \\
13.000 \\
13.400\end{array}$ & $\begin{array}{l}.6600320+00 \\
.6629161+00 \\
.6655298+00 \\
.6679009+00 \\
.6700540+00\end{array}$ & $\begin{array}{l}.1318898-01 \\
.1271108-01 \\
.1226548-01\end{array}$ & $\begin{array}{l}-.3411536-03 \\
-.3165021-03 \\
-.2944092-03 \\
-.2745348-03 \\
-.2565933-03\end{array}$ & $\begin{array}{l}.2124129-04 \\
.1922588-04 \\
.1745810-04\end{array}$ & $\begin{array}{l}.8937776-01 \\
.8368953-01 \\
.7837378-01 \\
.7339523-01 \\
.6872293-01\end{array}$ & $\begin{array}{l}-.1697097-01 \\
-.1625752-01 \\
-.1559883-01 \\
-.1498896-01 \\
-.1442281-01\end{array}$ & & & $\begin{array}{l}-.2630576+00 \\
-.2513088+00 \\
-.2403359+00 \\
-.2300644+00 \\
-.2204289+00\end{array}$ & $\begin{array}{l}.3889046-01 \\
.3715025-01 \\
.3554987-01 \\
.3407359-01 \\
.3270797-01\end{array}$ & $\begin{array}{l}-.2575113-02 \\
-.2374980-02 \\
-.2196869-02 \\
-.2037704-02 \\
-.1894926-02\end{array}$ & $\begin{array}{l}.3604383-03 \\
.3223575-03 \\
.2894421-03 \\
.2608482-03 \\
.2358913-03\end{array}$ \\
\hline $\begin{array}{l}13.800 \\
14.200 \\
14.600 \\
15.000 \\
16.000\end{array}$ & $\begin{array}{l}.6720105+00 \\
.6737892+00 \\
.6754068+00 \\
.6768780+00 \\
.6799984+00\end{array}$ & $\begin{array}{l}.1184905-01 \\
.1145906-01 \\
.1109311-01 \\
.1074908-01 \\
.9972997-02\end{array}$ & $\begin{array}{l}-.2403433-03 \\
-.2255798-03 \\
-.2121279-03 \\
-.1998374-03 \\
-.1733864-03\end{array}$ & $\begin{array}{l}.1590104-04 \\
.1452421-04 \\
.1330226-04 \\
.1221400-04 \\
.9968084-05\end{array}$ & $\begin{array}{l}.6432962-01 \\
.6019117-01 \\
.5628617-01 \\
.5259553-01 \\
.4419912-01\end{array}$ & $\begin{array}{l}-.1389594-01 \\
-.1340448-01 \\
-.1294507-01 \\
-.1251473-01 \\
-.1154963-01\end{array}$ & $\begin{array}{l}.5338964-03 \\
.4998668-03 \\
.4689529-03 \\
.4407889-03 \\
.3804539-03\end{array}$ & $\begin{array}{l}-.5045034-04 \\
-.4599170-04 \\
-.4204357-04 \\
-.3853502-04 \\
-.3131921-04\end{array}$ & $\begin{array}{l}-.2113720+00 \\
-.2028431+00 \\
-.1947972+00 \\
-.1871943+00 \\
-.1699006+00\end{array}$ & \begin{tabular}{l|}
$.3144136-01$ \\
$.3026368-01$ \\
$.2916615-01$ \\
$.2814108-01$ \\
$.2585306-01$
\end{tabular} & $\begin{array}{l}-.1766387-02 \\
-.1650279-02 \\
-.1545068-02 \\
-.1449447-02 \\
-.1245398-02\end{array}$ & $\begin{array}{l}.2140128-03 \\
.1947536-03 \\
.1777342-03 \\
.1626389-03 \\
.1316895-03\end{array}$ \\
\hline $\begin{array}{l}17.000 \\
18.000 \\
19.000 \\
20.000 \\
21.000\end{array}$ & $\begin{array}{l}.6824518+00 \\
.6843676+00 \\
.6858456+00 \\
.6869644+00 \\
.6877861+00\end{array}$ & $\begin{array}{l}.9298383-02 \\
.8706784-02 \\
.8183938-02 \\
.7718651-02 \\
.7302021-02\end{array}$ & $\begin{array}{l}-.1518376-03 \\
-.1340547-03 \\
-.1192118-03 \\
-.1066967-03 \\
-.9604845-04\end{array}$ & $\begin{array}{l}.5825467-05 \\
.4967610-05 \\
.4270889-05\end{array}$ & $\begin{array}{l}.3681811-01 \\
.3028026-01 \\
.2445020-01 \\
.1922006-01 \\
.1450284-01\end{array}$ & $\begin{array}{l}-.1071722-01 \\
-.9992375-02 \\
-.9355849-02 \\
-.8792692-02 \\
-.8291116-02\end{array}$ & $\begin{array}{l}.3316054-03 \\
.2915206-03 \\
.2582343-03 \\
.2303005-03 \\
.2066365-03\end{array}$ & $\begin{array}{l}-.2580010-04 \\
-.2150705-04 \\
-.1811768-04 \\
-.1540616-04 \\
-.1321109-04\end{array}$ & $\begin{array}{l}-.1546989+00 \\
-.1412313+00 \\
-.1292170+00 \\
-.1184330+00 \\
-.1086997+00\end{array}$ & $\begin{array}{l}.2389209-01 \\
.2219427-01 \\
.2071108-01 \\
.1940511-01 \\
.1824705-01\end{array}$ & $\begin{array}{l}-.1081064-02 \\
-.9468569-03 \\
-.8358995-03 \\
-.7431583-03 \\
-.6648840-03\end{array}$ & $\begin{array}{l}.1081167-03 \\
.8985027-04 \\
.7547890-04 \\
.6401820-04 \\
.5476749-04\end{array}$ \\
\hline $\begin{array}{l}22.000 \\
23.000 \\
24.000 \\
25.000 \\
26.000\end{array}$ & $\begin{array}{l}.6883605+00 \\
.6887279+00 \\
.6889213+00 \\
.6889677+00 \\
.6888894+00\end{array}$ & $\begin{array}{l}.6926877-02 \\
.6587384-02 \\
.6278745-02 \\
.5996982-02 \\
.5738767-02\end{array}$ & $\begin{array}{l}-.8691435-04 \\
-.7902113-04 \\
-.7215432-04 \\
-.6614378-04 \\
-.6085314-04\end{array}$ & $\begin{array}{l}.3699121-05 \\
.3225457-05 \\
.2829689-05 \\
.2496409-05 \\
.2213730-05\end{array}$ & $\begin{array}{r}.1022756-01 \\
.6335746-02 \\
.2778805-02 \\
-.4839969-03 \\
-.3487056-02\end{array}$ & $\begin{array}{l}-.7841702-02 \\
-.7436844-02 \\
-.7070329-02 \\
-.6737040-02 \\
-.6432721-02\end{array}$ & $\begin{array}{l}.1864188-03 \\
.1690126-03 \\
.1539223-03 \\
.1407563-03 \\
.1292023-03\end{array}$ & $\begin{array}{l}-.1141509-04 \\
-.9931364-05 \\
-.8694835-05 \\
-.7656039-05 \\
-.6776934-05\end{array}$ & $\begin{array}{l}-.9987096-01 \\
-.9182649-01 \\
-.8446653-01 \\
-.7770756-01 \\
-.7147914-01\end{array}$ & $\begin{array}{l}.1721361-01 \\
.1628612-01 \\
.1544939-01 \\
.1469098-01 \\
.1400059-01\end{array}$ & $\begin{array}{l}-.5982388-03 \\
-.5410445-03 \\
-.4916071-03 \\
-.4485939-03 \\
-.4109453-03\end{array}$ & $\begin{array}{l}.4721897-04 \\
.4099854-04 \\
.3582651-04 \\
.3149096-04 \\
.2782935-04\end{array}$ \\
\hline $\begin{array}{l}27.000 \\
28.000 \\
29.000 \\
30.000 \\
32.000\end{array}$ & $\begin{array}{l}.6887051+00 \\
.6884305+00 \\
.6880786+00 \\
.6876608+00 \\
.6866635+00\end{array}$ & $\begin{array}{l}.5501299-02 \\
.5282199-02 \\
.5079439-02 \\
.4891273-02 \\
.4552907-02\end{array}$ & $\begin{array}{l}-.5617209-04 \\
-.5201061-04 \\
-.4829470-04 \\
-.4496305-04 \\
-.3925618-04\end{array}$ & $\begin{array}{l}.1972380-05 \\
.1765058-05 \\
.1585956-05 \\
.1430421-05 \\
.1175699-05\end{array}$ & $\begin{array}{l}-.6259617-02 \\
-.8826701-02 \\
-.1120984-01 \\
-.1342769-01 \\
-.1743025-01\end{array}$ & $\begin{array}{l}-.6153809-02 \\
-.5897295-02 \\
-.5660624-02 \\
-.5441613-02 \\
-.5049311-02\end{array}$ & $\begin{array}{l}.1099703-03 \\
.1019201-03 \\
.9471953-04 \\
.8242556-04\end{array}$ & $\begin{array}{l}-.6027937-05 \\
-.5385806-05 \\
-.4832112-05 \\
-.4352113-05 \\
-.3567890-05\end{array}$ & $\begin{array}{l}-.6572141-01 \\
-.6038325-01 \\
-.5542065-01 \\
-.5079556-01 \\
-.4242986-01\end{array}$ & $\begin{array}{l}.1336963-01 \\
.1279089-01 \\
.1225826-01 \\
.1176653-01 \\
.1088861-01\end{array}$ & $\begin{array}{l}-.3778102-03 \\
-.3484991-03 \\
-.3224489-03 \\
-.2991956-03 \\
-.2596061-03\end{array}$ & $\begin{array}{l}.2471558-04 \\
.2205086-04 \\
.1975699-04 \\
.1777159-04 \\
.1453487-04\end{array}$ \\
\hline
\end{tabular}


$(m=24)$

\begin{tabular}{|c|c|c|c|c|c|c|c|c|c|c|c|c|}
\hline $\mathrm{T}^{*}$ & $\mathrm{~B}^{*(0)}$ & $\mathrm{B}^{*(1)}$ & $\mathrm{B}^{*(2)}$ & $\mathrm{B}^{*(3)}$ & $T^{*} \frac{\partial B^{*(0)}}{\partial T^{*}}$ & $\mathrm{~T}^{*} \frac{\partial \mathrm{B}^{*(1)}}{\partial \mathrm{T}^{*}}$ & $\mathrm{~T}^{*} \frac{\partial \mathrm{B}^{*(2)}}{\partial \mathrm{T}^{*}}$ & $\mathrm{~T}^{*} \frac{\partial \mathrm{B}^{*(3)}}{\partial \mathrm{T}^{*}}$ & $T^{* 2} \frac{\partial^{2} B^{*(0)}}{\partial T^{* 2}}$ & $\mathrm{~T} * 2 \frac{\partial^{2} \mathrm{~B}^{*(1)}}{\partial \mathrm{T}^{* 2}}$ & $T^{* 2} \frac{\partial^{2} B^{*(2)}}{\partial T^{* 2}}$ & $\mathrm{~T}^{* 2} \frac{\partial^{2} \mathrm{~B}^{*(3)}}{\partial \mathrm{T}^{* 2}}$ \\
\hline & (1) & & & & & & & & & & & \\
\hline & & & & $0211-06$ & $2404398-01$ & $.4408955-02$ & & $.2486603-05$ & $-.2854121-01$ & $.9463735-02$ & $007223-03$ & $.1008894-04$ \\
\hline & & -02 & 04 & $0938-06$ & 01 & & & .210814 & $271610-01$ & $78227-02$ & $3-03$ & $.8538444-05$ \\
\hline & 00 & $9170-02$ & $163208-04$ & $39891-06$ & $.2927212-01$ & $-.3908995-02$ & $.5118840-04$ & $-.1803127-05$ & $-.1748651-01$ & $.8358643-02$ & $-.1597546-03$ & $.7291337-05$ \\
\hline 42.000 & 00 & -02 & 04 & $72310-06$ & $.3149201-01$ & 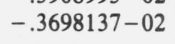 & .46162 & $.1554517-05$ & $-.1276663-01$ & $.7894598-02$ & $438074-03$ & .627677 \\
\hline 44.000 & $6+00$ & $.3206064-02$ & 04 & 06 & $49761-01$ & $-.3508250-02$ & $.4184176-04$ & $-.1349827-05$ & $-.8486337-02$ & $.7477763-02$ & $-.1301273-03$ & $.5442877-05$ \\
\hline & & & & & & & & & -. & & & $x_{2}+2>0$ \\
\hline & & & & & & & & & $\begin{array}{lll}- & & \\
\end{array}$ & & & \\
\hline & & & & & 10006 & & & $-.9169234-06$ & $48583-02$ & .644878 & $01812-04$ & $84312-05$ \\
\hline 52.000 & 00 & .267 & -04 & -06 &  & 年 & -04 & $46279-06$ & 02 & $74-02$ & $9109399-04$ & 05 \\
\hline .00 & $.6707282+00$ & $564482-02$ & -04 & .2437295 & $-.4115719-01$ & -.278 & .2722 & .727092 & $.8043202-02$ & $.5902876-02$ & -.8408294 & .291579 \\
\hline & & & & & & & & & & & & \\
\hline & & & & & & & & & -01 & & 04 & $7969-05$ \\
\hline & & & & & & & & & & & & \\
\hline & & & & & .4 & 02 & -04 & 6 & $316-01$ & $.4863734-02$ & -.5 & .17 \\
\hline 68.0 & $.6604363+00$ & 02 & 82678 & .1227 & 7824 & 02 & .16858 & 0 & A & 02 & - & .145 \\
\hline & & & & & & & & & & & & \\
\hline & & & & & & & & & & & & \\
\hline 80 & & & & & & & & & & & & \\
\hline & & 0 & $86-05$ & -07 & 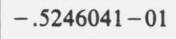 & .17087 & $8-04$ & -.19399 & .3 & 02 &.- & .7 \\
\hline 88 & & & -4 & 07 & .5331946 & -.16238 & .99 & -.16895 & .35 & .340 & -.302 & .670605 \\
\hline & & & & & & & & & & & & \\
\hline & & & & & & & & & & & & .5 \\
\hline & & & & & & & & & & & & .45 \\
\hline & & & 5 & $5-07$ & 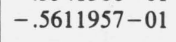 & 2 & .69 & 6 & .4 & 02 & -.2 & .396183 \\
\hline 1 & & & & 7 & .567456 & -.12731 & .6 & 7 & .4 & .26 & - . & 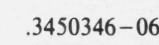 \\
\hline & & & & & & & & & & & & \\
\hline & & & 05 & & & & & & & & & \\
\hline & .627 & & & & & & & & & & & \\
\hline & & .9 & J & -01 & & & & & .4 & 02 & - . & -06 \\
\hline & & & & & 1 & 02 & & 7 & & & - . & .1880 \\
\hline & & & & & & & & & & & & \\
\hline & & & & & & & & & & & & \\
\hline & & & & & & & & & & & & \\
\hline & & .0 & $-.14 / 0530-05$ & $\cdot$ & $-.6038693-01$ & -.06 & .2 & 07 & $30-01$ & $80-02$ & $32-05$ & $.1139071-$ \\
\hline 1 & & & & 08 & -.6 & - . & .2 & -.2 & & & - . & .9 \\
\hline & & & & & & & & & & & & \\
\hline & & & & & 1 & & & & & & - . & $9851-07$ \\
\hline & & & & & & & & & & & & \\
\hline & $.5964105+00$ & $\cdot 0$ & (1) & 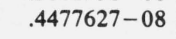 & $.01 / 1091-$ & $-.0345349-$ & $.1702853-$ & 07 & $.5822464-01$ & $.1312843-02$ & $-.5119091-05$ & .5120775 \\
\hline & & & & & & & & & & . &.- & .44 \\
\hline & & & & & & & & & & & & \\
\hline & & & & & & & & & .6 & 02 & -.3 & $9274-07$ \\
\hline & $.5785301+00$ & & a 10 & & & & & & & & & \\
\hline & 229 & .4117423 & $203010-$ & $.1586246-c$ & $.6245020-01$ & $003-03$ & $.8342906-06$ & $.4608115-08$ & $.6204081-01$ & $.8949331-03$ & $-.2498724-05$ & $.1803057-07$ \\
\hline
\end{tabular}


$(m=36)$

\begin{tabular}{|c|c|c|c|c|c|c|c|c|c|c|c|c|}
\hline$T^{*}$ & B & 1) & $B^{*(2)}$ & $\mathrm{B}^{*(3)}$ & $\mathrm{T}^{*} \frac{\partial \mathrm{B}^{*(0)}}{\partial \mathrm{T}^{*}}$ & $\mathrm{~T}^{*} \frac{\partial \mathrm{B}^{*(1)}}{\partial \mathrm{T}^{*}}$ & $\mathrm{~T}^{*} \frac{\partial \mathrm{B}^{*(2)}}{\partial \mathrm{T}^{*}}$ & $\mathrm{~T}^{*} \frac{\partial \mathrm{B}^{*(3)}}{\partial \mathrm{T}^{*}}$ & $\mathrm{~T}^{* 2} \frac{\partial^{2} \mathrm{~B}^{*(0)}}{\partial \mathrm{T}^{* 2}}$ & $\mathrm{~T}^{* 2} \frac{\partial^{2} \mathrm{~B}^{*(1)}}{\partial \mathrm{T}^{* 2}}$ & $T^{* 2} \frac{\partial^{2} B^{*(2)}}{\partial T^{* 2}}$ & $\Gamma * 2 \frac{\partial^{2} B *(3)}{\partial T^{* 2}}$ \\
\hline $\begin{array}{l}.300 \\
.400 \\
.500 \\
.600 \\
.700\end{array}$ & $\begin{array}{l}-.1465943+02 \\
-.7189372+01 \\
-.4444152+01 \\
-.3062228+01 \\
-.2238775+01\end{array}$ & $\begin{array}{l}.4555752+02 \\
.1323856+02 \\
.5918915+01 \\
.3323108+01 \\
.2139180+01\end{array}$ & $\begin{array}{l}-.2487489+03 \\
-.4612313+02 \\
-.1471505+02 \\
-.6313966+01 \\
-.3253358+01\end{array}$ & $\begin{array}{l}.2020919+04 \\
.2577075+03 \\
.6229556+02 \\
.2146971+02 \\
.9236215+01\end{array}$ & $\begin{array}{l}.4028437+02 \\
.1624736+02 \\
.9223124+01 \\
.6199639+01 \\
.4589943+01\end{array}$ & $\begin{array}{l}-.2163288+03 \\
-.5138936+02 \\
-.1983524+02 \\
-.9939588+01 \\
-.5839908+01\end{array}$ & $\begin{array}{l}.1576807+04 \\
.2497712+03 \\
.7125826+02 \\
.2809095+02 \\
.1353433+02\end{array}$ & $\begin{array}{l}-.1550987+05 \\
-.1722306+04 \\
-.3776697+03 \\
-.1209487+03 \\
-.4910982+02\end{array}$ & $\begin{array}{l}-.1847960+03 \\
-.6132110+02 \\
-.3065272+02 \\
-.1888310+02 \\
-.1313352+02\end{array}$ & $\begin{array}{l}.1400035+04 \\
.2856811+03 \\
.9899528+02 \\
.4570279+02 \\
.2515650+02\end{array}$ & $\begin{array}{l}-.1247695+05 \\
-.1732523+04 \\
-.4506032+03 \\
-.1656777+03 \\
-.7555459+02\end{array}$ & $\begin{array}{l}.1423912+06 \\
.1401791+05 \\
.2825119+04 \\
.8491768+03 \\
.3280311+03\end{array}$ \\
\hline $\begin{array}{l}.750 \\
.775 \\
.800 \\
.825 \\
.850\end{array}$ & $\begin{array}{l}-.1941354+01 \\
-.1812463+01 \\
-.1694559+01 \\
-.1586307+01 \\
-.1486580+01\end{array}$ & $\begin{array}{l}.1778463+01 \\
.1633099+01 \\
.1505898+01 \\
.1393944+01 \\
.1294882+01\end{array}$ & $\begin{array}{l}-.2451678+01 \\
-.2149234+01 \\
-.1895129+01 \\
-.1680093+01 \\
-.1496900+01\end{array}$ & $\begin{array}{l}.6428836+01 \\
.5428481+01 \\
.4616862+01 \\
.3952786+01 \\
.3405157+01\end{array}$ & & $\begin{array}{l}-.4667631+01 \\
-.4208021+01 \\
-.3812570+01 \\
-.3470122+01 \\
-.3171800+01\end{array}$ & $\begin{array}{l}.9910369+01 \\
.8572389+01 \\
.7462902+01 \\
.6535725+01 \\
.5755310+01\end{array}$ & $\begin{array}{l}-.3335124+02 \\
-.2784138+02 \\
-.2342179+02 \\
-.1984489+02 \\
-.1692588+02\end{array}$ & $\begin{array}{l}-.1129532+02 \\
-.1053831+02 \\
-.9866837+01 \\
-.9268002+01 \\
-.8731284+01\end{array}$ & $\begin{array}{l}.1955099+02 \\
.1739696+02 \\
.1556618+02 \\
.1399930+02 \\
.1264962+02\end{array}$ & $\begin{array}{l}-.5404033+02 \\
-.4623680+02 \\
-.3983499+02 \\
-.3453977+02 \\
-.3012637+02\end{array}$ & $\begin{array}{l}.2180897+03 \\
.1802724+03 \\
.1502347+03 \\
.1261515+03 \\
.1066737+03\end{array}$ \\
\hline $\begin{array}{l}.875 \\
.900 \\
.925 \\
.950 \\
.975\end{array}$ & $\begin{array}{l}-.1394417+01 \\
-.1308997+01 \\
-.1229612+01 \\
-.1155649+01 \\
-.1086576+01\end{array}$ & & $\begin{array}{l}-.9848654+00 \\
-.8953654+00\end{array}$ & & $\begin{array}{l}.3103262+01 \\
.2962556+01 \\
.2833423+01 \\
.2714532+01 \\
.2604743+01\end{array}$ & $\begin{array}{l}-.2910477+01 \\
-.2680381+01 \\
-.2476808+01 \\
-.2295892+01 \\
-.2134434+01\end{array}$ & $\begin{array}{l}.5094024+01 \\
.4530187+01 \\
.4046637+01 \\
.3629686+01 \\
.3268327+01\end{array}$ & & $\begin{array}{l}-.8248018+01 \\
-.7811020+01 \\
-.7414291+01 \\
-.7052787+01 \\
-.6722243+01\end{array}$ & & $\begin{array}{l}-.2642181+02 \\
-.2329168+02 \\
-.2063057+02 \\
-.1835516+02 \\
-.1639901+02\end{array}$ & $\begin{array}{l}.9079276+02 \\
.7774649+02 \\
.6695318+02 \\
.5796472+02 \\
.5043290+02\end{array}$ \\
\hline $\begin{array}{l}1.000 \\
1.020 \\
1.040 \\
1.060 \\
1.080\end{array}$ & $\begin{array}{l}-.8824791+00 \\
-.8403271+00\end{array}$ & $\begin{array}{l}.8841885+00 \\
.8458378+00 \\
.8101914+00 \\
.7769955+00 \\
.7460256+00\end{array}$ & $-8166755+00$ & & & & & & & & $\begin{array}{l}-.1470879+02 \\
-.1351883+02 \\
-.1245302+02 \\
-.1149577+02 \\
-.1063376+02\end{array}$ & $\begin{array}{l}.3978261+02 \\
.3586981+02 \\
.3248169+02 \\
.2948541+02\end{array}$ \\
\hline $\begin{array}{l}1.100 \\
1.120 \\
1.140 \\
1.160 \\
1.180\end{array}$ & $-.6559964+00$ & $\begin{array}{l}.7170823+00 \\
.6899884+00 \\
.6645854+00 \\
.6407318+00 \\
.6183006+00\end{array}$ & $\begin{array}{l}-.4841352+00 \\
-.4566195+00\end{array}$ & & & & & & & & & $\begin{array}{l}.2446460+02 \\
.2235710+02 \\
.2047301+02 \\
.1878454+02\end{array}$ \\
\hline $\begin{array}{l}1.200 \\
1.220 \\
1.240 \\
1.260 \\
1.280\end{array}$ & $\begin{array}{l}-.5926344+00 \\
-.5628285+00 \\
-.5341768+00 \\
-.5066144+00\end{array}$ & $\begin{array}{l}.5971777+00 \\
.5772601+00 \\
.5584550+00 \\
.5406784+00 \\
.5238542+00\end{array}$ & $4312327+00$ & & & & & & & & & $\begin{array}{l}.1590232+02 \\
.1467030+02 \\
.1355639+02 \\
.1254724+02\end{array}$ \\
\hline $\begin{array}{l}1.300 \\
1.320 \\
1.340 \\
1.360 \\
1.380\end{array}$ & $\begin{array}{l}-.4800807+00 \\
-.4545200+00 \\
-.4298801+00 \\
-.4061127+00 \\
-.3831728+00\end{array}$ & $\begin{array}{l}.4927926+00 \\
.4784348+00 \\
.4647876+00 \\
.4518031+00\end{array}$ & $\begin{array}{l}-.2844215+00 \\
-.2712370+00\end{array}$ & & & & & & & & & $\begin{array}{l}.1079824+02 \\
.1003934+02 \\
.9346770+01 \\
.8713660+01\end{array}$ \\
\hline $\begin{array}{l}1.400 \\
1.420 \\
1.440 \\
1.460 \\
1.480\end{array}$ & $\begin{array}{l}-.3390102+00 \\
-.3189116+00 \\
-.2988883+00 \\
-.2795082+00\end{array}$ & $\begin{array}{l}.4394373+00 \\
.4276500+00 \\
.4164043+00 \\
.4056660+00 \\
.3954038+00\end{array}$ & $\begin{array}{l}-.2364760+00 \\
-.2262821+00 \\
-.2166968+00\end{array}$ & & & & & & & & & $\begin{array}{l}. .7602361+01 \\
.7114106+01 \\
.6665012+01 \\
.6251351+01\end{array}$ \\
\hline $\begin{array}{l}1.500 \\
1.540 \\
1.580 \\
1.620 \\
1.660\end{array}$ & $\begin{array}{l}-.2607412+00 \\
-.2249348+00 \\
-.1912628+00 \\
-.1595421+00 \\
-.1296100+00\end{array}$ & $\begin{array}{l}.3671951+00 \\
.3502941+00 \\
.3347216+00 \\
.3203355+00\end{array}$ & $\begin{array}{l}-.1632667+00 \\
-.1514501+00\end{array}$ & $\begin{array}{l}.2047962+00 \\
.1846054+00 \\
.1669262+00\end{array}$ & & $\begin{array}{l}-.7200417+00 \\
-.6782872+00 \\
-.6403500+00 \\
-.6057724+00 \\
-.5741633+00\end{array}$ & $\begin{array}{l}.5996838+00 \\
.5493326+00 \\
.5046516+00 \\
.4648562+00\end{array}$ & $\begin{array}{l}-.9551452+00 \\
-.8527877+00 \\
-.7641118+00 \\
-.6869560+00\end{array}$ & $\begin{array}{l}-.3160657+01 \\
-.3041937+01 \\
-.2931440+01 \\
-.2828358+01\end{array}$ & & $\begin{array}{l}-.2930228+01 \\
-.2658867+01 \\
-.2420579+01 \\
-.2210467+01 \\
-.2024473+01\end{array}$ & $\begin{array}{l}.5191515+01 \\
.4609999+01 \\
.4109052+01 \\
.3675554+01\end{array}$ \\
\hline $\begin{array}{l}1.700 \\
1.740 \\
1.780 \\
1.820 \\
1.860\end{array}$ & $\begin{array}{l}-.1013214+00 \\
-.7454647-01 \\
-.4916856-01 \\
-.2508273-01 \\
-.2194258-02\end{array}$ & $\begin{array}{l}.3070128+00 \\
.2946463+00 \\
.2831420+00 \\
.2724177+00 \\
.2624007+00\end{array}$ & $\begin{array}{l}-.1408111+00 \\
-.1312033+00 \\
-.1225016+00 \\
-.1145991+00 \\
-.1074038+00\end{array}$ & $\begin{array}{l}.1255412+00 \\
.1147623+00 \\
.1051591+00\end{array}$ & $\begin{array}{l}.1133524+01 \\
.1099830+01 \\
.1067983+01 \\
.1037836+01\end{array}$ & $\begin{array}{l}-.5451871+00 \\
-.5185543+00 \\
-.4940141+00 \\
-.4713486+00 \\
-.4503677+00\end{array}$ & $\begin{array}{l}.32573958+00 \\
.3687086+00 \\
.3428286+00 \\
.3194160+00\end{array}$ & $\begin{array}{l}-.6195491+00 \\
-.5604315+00 \\
-.5083940+00 \\
-.4624293+00 \\
-.4216948+00\end{array}$ & $\begin{array}{l}-.2731986+01 \\
-.2641704+01 \\
-.2556962+01 \\
-.2477276+01 \\
-.2402215+01\end{array}$ & $\begin{array}{l}.1724976+01 \\
.1629877+01 \\
.1542833+01 \\
.1462957+01 \\
1389481+01\end{array}$ & $\begin{array}{l}-.1859225+01 \\
-.1711894+01 \\
-.1580107+01 \\
-.1461853+01 \\
-.1355429+01\end{array}$ & $\begin{array}{l}.2970083+01 \\
.2682135+01 \\
.2428996+01 \\
.2205688+01\end{array}$ \\
\hline
\end{tabular}


$(m=36)$

\begin{tabular}{|c|c|c|c|c|c|c|c|c|c|c|c|c|}
\hline $\mathrm{T}^{*}$ & $B^{*(0)}$ & $\mathrm{B}^{*(1)}$ & $\mathrm{B}^{*(2)}$ & $\mathrm{B}^{*(3)}$ & $\mathrm{T}^{*} \frac{\partial \mathrm{B} *(0)}{\partial \mathrm{T}^{*}}$ & $\mathrm{~T}^{*} \frac{\partial \mathrm{B} *(1)}{\partial \mathrm{T}^{*}}$ & $\mathrm{~T}^{*} \frac{\partial \mathrm{B}^{*(2)}}{\partial \mathrm{T}^{*}}$ & $\mathrm{~T}^{*} \frac{\partial \mathrm{B} *(3)}{\partial \mathrm{T}^{*}}$ & $\mathrm{~T}^{* 2} \frac{\partial^{2} \mathrm{~B}^{*(0)}}{\partial \mathrm{T}^{* 2}}$ & $\mathrm{~T}^{* 2} \frac{\partial^{2} \mathrm{~B}^{*(1)}}{\partial \mathrm{T}^{* 2}}$ & $\mathrm{~T}^{* 2} \frac{\partial^{2} \mathrm{~B}^{*(2)}}{\partial \mathrm{T}^{* 2}}$ & $\mathrm{~T}^{* 2} \frac{\partial^{2} \mathrm{~B}^{*(3)}}{\partial \mathrm{T}^{* 2}}$ \\
\hline 1.900 & $.1958257-01$ & $30269+00$ & $1008362+00$ & $.9657785-01$ & $.1009258+01$ & $-.4309047+00$ & $.2981790+00$ & $-.3854820+00$ & $-.2331394+01$ & $.1321735+01$ & $-.1259381+01$ & $.2008050+01$ \\
\hline & $.4032551-01$ & $42395+00$ & $9482753-01$ & & $.9821300+00$ & $-.4128133+00$ & $.2788667+00$ & $-.3531931+00$ & $-.2264471+01$ & $.1259135+01$ & & $.1832584+01$ \\
\hline 1.980 & $10525-01$ & $59878+00$ & $.8931794-01$ & $97900-01$ & $.9563444+00$ & $-.3959644+00$ & $.2612624+00$ & $-.3243207+00$ & $-.2201137+01$ & $.1201170+01$ & $-.1093612+01$ & $.1676339+01$ \\
\hline 2.020 & $.7898618-01$ & $.2282267+00$ & $.8425522-01$ & $.7575504-01$ & $.9318047+00$ & $-.3802440+00$ & $.2451781+00$ & $-.2984334+00$ & $-.2141117+01$ & $.1147390+01$ & $-.1021900+01$ & $.1536815+01$ \\
\hline 2.060 & $.9702714-01$ & $.2209159+00$ & $-.7959367-01$ & $.7013463-01$ & $.9084230+00$ & $-.3655507+00$ & $.2304504+00$ & $-.2751623+00$ & $-.2084160+01$ & $.1097396+01$ & $-.9565298+00$ & $.1411884+01$ \\
\hline 2.100 & $.1142819+00$ & $.2140191+00$ & $-.7529316-01$ & $.6504734-01$ & $.8861196+00$ & $-.3517945+00$ & $.2169365+00$ & $-.2541913+00$ & $-.2030041+01$ & $.1050840+01$ & $-.8968091+00$ & $.1299730+01$ \\
\hline 2.140 & $.1307998+00$ & $5039+00$ & .7131837 & & +00 & $388951+00$ & & $-.2352482+00$ & $8556+01$ & $7409+01$ & $21338+00$ & $.1198797+01$ \\
\hline 2.180 & $.1466262+00$ & $.2013409+00$ & .6763 & .56236 & $4636+00$ & $67806+00$ & $0659+00$ & $-.2180981+00$ & $99520+01$ & $8266+00$ & $919758+00$ & $.1107746+01$ \\
\hline 2.220 & $.1618026+00$ & $.1955036+00$ & $-.6422473-01$ & $.5241440-01$ & $.8249840+00$ & $-.3153868+00$ & $.1825030+00$ & $-.2025375+00$ & $-.1882765+01$ & $.9288456+00$ & $-.7458725+00$ & $.1025424+01$ \\
\hline & $.1763676+00$ & $.1899680+00$ & $-.6105372-01$ & $.4892549-01$ & $.8063277+00$ & $-.3046557+00$ & $.1727376+00$ & $-.1883896+00$ & $-.1838137+01$ & $.8932452+00$ & $-.7034171+00$ & $.9508317+00$ \\
\hline 2.300 & $.1903566+00$ & $.1847124+00$ & $-.5810326-01$ & $.4573480-01$ & $.7884435+00$ & $-.2945353+00$ & $.1636942+00$ & $-.1755005+00$ & $-.1795496+01$ & $.8598279+00$ & $-.6642509+00$ & $.8831011+00$ \\
\hline & & .179 & -01 & .42811 & $.7712848+00$ & $-.2849785+00$ & & $-.1637355+00$ & $-.1754714+01$ & $.8284158+00$ & $-.6280572+00$ & $.8214778+00$ \\
\hline 2.380 & $.2167350+00$ & $.1749638+00$ & .5278 & & $2+00$ & $8+00$ & $0+00$ & $768+00$ & $5673+01$ & $8491+00$ & +00 & $.7653028+00$ \\
\hline 2.42 & $.2291830+00$ & $.1704364+00$ & $.5039054-01$ & .376 & -00 & +00 & +00 & $31208+00$ & $265+01$ & $.7709835+00$ & $-.5634952+00$ & $.7139992+00$ \\
\hline 2.460 & $.2411723+00$ & $97+00$ & $7-01$ & -01 & $.7237455+00$ & $2839+00$ & $.1335069+00$ & $-.1340762+00$ & $-.1642391+01$ & $.7446886+00$ & $-.5346556+00$ & $.6670608+00$ \\
\hline 2.500 & $.2527272+00$ & $.1620000+00$ & $-.4604487-01$ & .3329 & $.7090885+00$ & $-.2515939+00$ & $.1272036+00$ & $-.1257627+00$ & $-.1607959+01$ & $.7198462+00$ & $-.5078381+00$ & $.6240422+00$ \\
\hline & & & & & & & & & +01 & +00 & $41+00$ & .53117 \\
\hline 2.700 & +00 & .1439 & .372 & & .64334 & $-.2184464+00$ & +00 & -01 & $692+01$ & +00 & $854+00$ & .45547 \\
\hline 2.800 & .3275 & $.1362590+00$ & 01 & & $.6145347+00$ & $-.2046100+00$ & $5326-01$ & $-.8067691-01$ & $-.1388124+01$ & $.5704360+00$ & $-.3559486+00$ & $.3932048+00$ \\
\hline 2.900 & $.3486912+00$ & $.1292987+00$ & $-.3076310-01$ & $.1914862-01$ & $.5879982+00$ & $22396+00$ & $5183-01$ & $-.7045473-01$ & $-.1327149+01$ & $.5318353+00$ & $-.3195349+00$ & $.3415629+00$ \\
\hline 3.000 & $.3682066+00$ & $.1229720+00$ & -01 & .16 & -00 & $.1811267+00$ & 01 & -01 & 127109 & .497 & -.28 & 00 \\
\hline & & & & & & & & & & & & \\
\hline 3.200 & .40 & 00 & 01 & & -00 & 00 & & -. & 01 & +00 & $2675+00$ & .23132 \\
\hline 3.300 & +00 & +00 & 01 & & & & & & & .4 & & +00 \\
\hline 3.400 & $.4334733+00$ & $.1025795+00$ & $-.2024429-01$ & $.1074564-01$ & $.4815808+00$ & $-.1462112+00$ & $942-01$ & $43-01$ & $866+01$ & $.3913474+00$ & $-.1982286+00$ & $.1826472+00$ \\
\hline 3.500 & $.4471822+00$ & $.9844222-01$ & $-.1878430-01$ & $.968 c$ & .464 & .13 & -01 & -.3 & -.10 & .37 & -.182068 & .16329 \\
\hline & & & & & & & & & & & & \\
\hline 3. & .47 & & & & & & & 01 & 00 & +00 & +00 & $.1319524+00$ \\
\hline 3.800 & .4834 & .87 & 01 & & & & & & & +00 & & \\
\hline 3.900 & $.4941668+00$ & $.8463175-01$ & $-.1425535-01$ & $.6601897-02$ & $.4052924+00$ & $-.1167275+00$ & $.3598940-01$ & $-.2321061-01$ & $-.9161467+00$ & $.3043769+00$ & $-.1332147+00$ & $.1080429+00$ \\
\hline 4.000 & $.5042659+00$ & $.8173566-01$ & .1337458-01 & .60405 & $.3925539+00$ & 11208 & .336161 & -.2 & 0 & .2 & 00 & 01 \\
\hline & & & & & & & & & $-.8615799+00$ & & & \\
\hline & $.5228402+00$ & $.7647334-01$ & $1-01$ & & & & & & $-.8365292+00$ & $9842+00$ & $57+00$ & $.8179306-01$ \\
\hline 4.300 & .5313 & .74 & -01 & & & & .2 & & & +00 & & $.7492927-01$ \\
\hline 4.400 & $.5395126+00$ & $.7181853-01$ & $1054592-01$ & $.4334933-02$ & $.3479124+00$ & $.9647432-01$ & $.2608277-01$ & $-.1499675-01$ & $-.7903163+00$ & $.2462835+00$ & $-.9487145-01$ & $.6880257-01$ \\
\hline 4.500 & .5472 & .69687 & $9976776-02$ & 4011128 & $.3381007+00$ & $.9317586-01$ & .245849 & -.138368 & -.7689580 & .236 & 01 & \\
\hline & & & & & & & & & & & & $.5839346-01$ \\
\hline 4.700 & $.5615223+00$ & $.6576848-01$ & $8966323-02$ & -02 & & & & & & & & -01 \\
\hline & .5681 & .635 & 2 & & & & & & & +00 & & $.4996062-01$ \\
\hline 4.900 & $.5745008+00$ & $.6224877-01$ & .8099689-02 & $.2994733-02$ & $.3031766+00$ & $-.8182452-01$ & $.1969358-01$ & $-.1022326-01$ & $-.6933111+00$ & $.2052377+00$ & $-.7059360-01$ & $.4634176-01$ \\
\hline 5.000 & $.5805468+00$ & $.6062058-01$ & $-.7712022-02$ & $2795388-02$ & $2953862+00$ & $-.7937523-01$ & .18693 & $-.9519945-02$ & & .1984 & & \\
\hline & & $.5907183-01$ & & & & $-.7705752-01$ & & -.8 & -.66 & & $3-01$ & $.4007823-01$ \\
\hline 5.200 & $.5918432+00$ & $.5759696-01$ & $2-02$ & & & & & &.$- t$ & & & \\
\hline & .59 & .561 & 2 & .22 & .27 & 01 & .161 & -.7 & -.6 & & -.5 & $408-01$ \\
\hline & & $.5484915-01$ & $6406388-02$ & .2153 & $37+00$ & $-.7079948-01$ & $337-01$ & $-.7269161-02$ & $-.6162436+00$ & $.1749609+00$ & $-.5435738-01$ & $.3261802-01$ \\
\hline 5.500 & $.6070305+00$ & .53567 & 2 & .20 & +00 & -.68 & .1 & 02 & -.6 & & $830-01$ & \\
\hline & & & & & & & & & & .16 & $-.4935412-01$ & $.2863720-01$ \\
\hline 5.80 & $.6204186+00$ & $.5004532-01$ & $-.5402722-02$ & $.1693424-02$ & $.2433651+00$ & $-.6379265-01$ & $.1281863-01$ & $-.5672268-02$ & $-.5651250+00$ & $.1560222+00$ & $-.4499177-01$ & $.2527341-01$ \\
\hline & $.6284865+00$ & $.4793465-01$ & $-.4986093-02$ & $947-02$ & $.2326627+00$ & $-.6075328-01$ & $.1177582-01$ & $-.5046441-02$ & $-.5423699+00$ & $.1478859+00$ & $-.4116745-01$ & $.2241240-01$ \\
\hline 6.200 & $.6359510+00$ & $.4598856-01$ & $-.4615318-02$ & $.1355458-02$ & $.2226920+00$ & $-.5797275-01$ & $.1085281-01$ & $|-.4508972-02|$ & $-.5212201+00$ & $.1404863+00$ & $-.3779785-01$ & $.1996425-01$ \\
\hline
\end{tabular}


$(m=36)$

\begin{tabular}{|c|c|c|c|c|c|c|c|c|c|c|c|c|}
\hline $\mathrm{T}^{*}$ & $\mathrm{~B}^{*(0)}$ & $\mathrm{B}^{*(1)}$ & $\mathrm{B}^{*(2)}$ & $\mathrm{B}^{*(3)}$ & $\mathrm{T}^{*} \frac{\partial \mathrm{B}^{*(0)}}{\partial \mathrm{T}^{*}}$ & $\mathrm{~T}^{*} \frac{\partial \mathrm{B}^{*(1)}}{\partial \mathrm{T}^{*}}$ & $\mathrm{~T}^{*} \frac{\partial \mathrm{B}^{*(2)}}{\partial \mathrm{T}^{*}}$ & $\mathrm{~T}^{*} \frac{\partial \mathrm{B}^{*(3)}}{\partial \mathrm{T}^{*}}$ & $\mathrm{~T}^{* 2} \frac{\partial^{2} \mathrm{~B}^{*(0)}}{\partial \mathrm{T}^{* 2}}$ & $\mathrm{~T}^{* 2} \frac{\partial^{2} \mathrm{~B}^{*(1)}}{\partial \mathrm{T}^{* 2}}$ & $\mathrm{~T}^{* 2} \frac{\partial^{2} \mathbf{B}^{*(2)}}{\partial \mathbf{T}^{* 2}}$ & $\mathrm{~T}^{* 2} \frac{\partial^{2} \mathrm{~B}^{*(3)}}{\partial \mathrm{T}^{* 2}}$ \\
\hline & & & 22 & & 00 & & -01 & -02 & 00 & +00 & -01 & .1785 \\
\hline & & & $86681-02$ & $01651-02$ & $46641+00$ & $5307024-01$ & $99527-02$ & $.3642080-02$ & $.4831002+00$ & $.1275462+00$ & $-.3216283-01$ & $.1603519-01$ \\
\hline 6.800 & $3+00$ & $96831-01$ & .3718991-02 & $.9982588-03$ & $64880+00$ & $-.5089985-01$ & $42883-02$ & $-.3290845-02$ & $-.4658630+00$ & $.1218626+00$ & $79517-01$ & $.1445102-01$ \\
\hline 7.000 & $08745+00$ & $52221-01$ & $.3477123-02$ & $.9073995-03$ & $388033+00$ & $-.4889001-01$ & $.8052218-02$ & $-.2983205-02$ & $-.4496905+00$ & $.1166252+00$ & $-.2767341-01$ & $.1306758-01$ \\
\hline 7.200 & $.6660908+00$ & $.3817141-01$ & $.3257886-02$ & & $315667+00$ & $-.4702411-01$ & $.7519091-02$ & $-.2712623-02$ & $-.4344867+00$ & $.1117856+00$ & $-.2576520-01$ & $.1185424-01$ \\
\hline 7.400 & $.6709716+00$ & $.3690696-01$ & $3058561-02$ & $.7562355-03$ & $.1747402+00$ & -.452876 & $.7036354-02$ & $-.2473703-02$ & $-.4201668+00$ & $.1073020+00$ & $-.2404329-01$ & $.1078577-0$ \\
\hline 7.600 & & & & & +00 & -43 & & & - & & & 128 \\
\hline 7.800 & $.6798369+00$ & $.3460646-01$ & $.2710685-02$ & & $21850+00$ & $-.4215421-01$ & $.6198613-02$ & $-.2073651-02$ & $-.3938870+00$ & $.9926195-01$ & $-.2106941-01$ & $.9003404-02$ \\
\hline 8.000 & $.6838695+00$ & .3355727 & 2 & & $33990+00$ & $-.4073640-01$ & $.5833944-02$ & $-.1905629-02$ & $-.3818004+00$ & $.9564622-01$ & $978094-01$ & $.8257564-02$ \\
\hline 8.200 & $.6876633+00$ & $.3256791-01$ & $.2418525-02$ & .54 & $.1509073+00$ & $-.3940602-01$ & $.5500071-02$ & $-.1755243-02$ & $-.3703428+00$ & $.9226641-01$ & $-.1860472-01$ & $.7591522-02$ \\
\hline 8.400 & $.6912366+00$ & $.3163348-01$ & $.2289716-02$ & .500697 & 00 & 1 & 02 & 02 & - & 01 & 01 & 02 \\
\hline 8.600 & & & & & & & & & & & & \\
\hline 8.800 & & & 2 & & +00 & & & -02 & - & $3959-01$ & $-.1563287-01$ & $.5976266-02$ \\
\hline 9.000 & & & & & & & & & & & & \\
\hline 9.200 & .70 & .28 & 02 & .373 & 00 & -01 & $.4189855-02$ & -.119974 & $-.3209643+00$ & $.7823363-01$ & $-.1402391-01$ & $.5145572-02$ \\
\hline 9.400 & .7 & $.2764653-01$ & $.1776748-02$ & .3485000 & +00 & $-.3288624-01$ & 02 & -.11178 & $-.3124207+00$ & $.7589365-01$ & $-.1330938-01$ & $.4787362-02$ \\
\hline 9.600 & & & & & & & & & & & & \\
\hline & & & & & & & & & & 01 & & \\
\hline 10.000 & & & 1 & & & & & -.5 & & -01 & & .38 \\
\hline 10.200 & $5+00$ & $.2509830-01$ & $.1480477-02$ & $.2683844-03$ & $.1082339+00$ & $-.2957704-01$ & $.3293710-02$ & $-.8558165-03$ & $-.2817684+00$ & $.6771273-01$ & $-.1092778-01$ & $.3645038-02$ \\
\hline 104 & $99+00$ & $.2453110-01$ & $-.1417937-02$ & $.2522808-03$ & $.1048988+00$ & .288468 & .31489 & -.80 & $-.2748788+00$ & .65 & -. & .34 \\
\hline & & & $-.1359256-02$ & & & & & & & & & \\
\hline 00 & & & 2 & & & & & & & & & \\
\hline & & & & & & & & & & & & $.2838496-02$ \\
\hline & & .2 & $57377-02$ & .18 & $3075-01$ & 5899-01 & $.2549414-02$ & $-.5965843-03$ & $-.2442185+00$ & $.5814925-01$ & $-.8384030-02$ & $.2523341-02$ \\
\hline 11.8 & & & - & $.1690288-03$ & .84 & 01 & .2356 & -.5337 & -.2334 & -01 & 02 & .225 \\
\hline & & & & & & & & & & & & \\
\hline & & 01 & 9 & & & - & & & & & & \\
\hline 13.000 & & & & & & & & & & & & .16421 \\
\hline & & $67-01$ & $866-03$ & .1 & 01 & 01 & .17 & -.3550833 & -.1 & $54-01$ & $-.5742305-02$ & $.1488213-02$ \\
\hline 13 & & .1 & -.76 & . & 01 & 01 & .16536 & $-.3233009-03$ & -.189409 & $.451 C$ & -.5 & .13 \\
\hline & & & & & & & & & & & & \\
\hline & & 01 & 03 & & & -1 & & & & & & .11 \\
\hline 15.000 & & & & & & & & & & & & \\
\hline & & $8-1$ & $1-00$ & $\cdot$ & 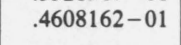 & -01 & .1 & 1 & & $177-01$ & $-.3821865-02$ & $.8404473-03$ \\
\hline 17.00 & .75 & -01 & 03 & & 01 & - & 2 & -.1 & - . & .345 & -02 & $.6924 S$ \\
\hline & & & & & & & & & & & & \\
\hline & & & 00 & & & & & & & & 2 & .4 \\
\hline 2000 & .7571 & & & & & & & & & & & \\
\hline & &. & 33 & - & $322-01$ & $1734-01$ & $.6557807-03$ & $-.8637679-04$ & $-.1017985+00$ & $.2652475-01$ & $-.2076301-02$ & .3547431 \\
\hline 22.000 & .75 & -01 & 03 & .2 & -01 & -.11 & .59 & -.7 & -.94255 & -01 & 02 & .30 \\
\hline & & & & & & & & & & & & \\
\hline & & & & 10 & .10 & 2 & .4 & 04 & -.0 & .22 & -02 & -03 \\
\hline & & & & & & & & & & & & \\
\hline & .7609 & .8 & $1-03$ & . & $.4967091-$ & - & .4142927 & $-.4463494-04$ & $-.6998168-01$ & $.2048532-01$ & $-.1298958-02$ & $.1819484-03$ \\
\hline 00 & .7 & .8 & -.18 & & & - & &.- & - . & -01 & & .1 \\
\hline & & & & & & & .3 & 4 & & & & \\
\hline & .76 & .7 & 157 & .1 & 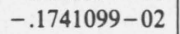 & .0 & .3 & 4 & - & $9487-01$ & $-.1025031-02$ & $.1296941-03$ \\
\hline & & .74 & & & $a^{2}$ & 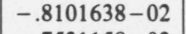 & .3056088 & 4 & & -01 & 03 & $.1167961-03$ \\
\hline 32.000 & $.7606734+00$ & $.6945208-02$ & $-.1287678-03$ & $.7817724-05$ & $.7162613-02$ & $-.7531158-02$ & $.2666376-03$ & $-.2362761-04$ & $-.4510349-01$ & $.1603093-01$ & $-.8292492-03$ & $.9572288-0$ \\
\hline
\end{tabular}


$(m=36)$

\begin{tabular}{|c|c|c|c|c|c|c|c|c|c|c|c|c|}
\hline $\mathrm{T}^{*}$ & $\mathrm{~B}^{*(0)}$ & $\mathrm{B}^{*(1)}$ & $\mathrm{B}^{*(2)}$ & $\mathrm{B}^{*(3)}$ & $\mathrm{T}^{*} \frac{\partial \mathrm{B}^{*(0)}}{\partial \mathrm{T}^{*}}$ & $\mathrm{~T}^{*} \frac{\partial \mathrm{B}^{*(1)}}{\partial \mathrm{T}^{*}}$ & $\mathrm{~T}^{*} \frac{\partial \mathrm{B}^{*(2)}}{\partial \mathrm{T}^{*}}$ & $\mathrm{~T}^{*} \frac{\partial \mathrm{B}^{*(3)}}{\partial \mathrm{T}^{*}}$ & $T^{* 2} \frac{\partial^{2} B^{*(0)}}{\partial T^{* 2}}$ & $\mathrm{~T}^{* 2} \frac{\partial^{2} \mathrm{~B}^{*(1)}}{\partial \mathrm{T}^{* 2}}$ & $\mathrm{~T}^{* 2} \frac{\partial^{2} \mathrm{~B}^{*(2)}}{\partial \mathrm{T}^{* 2}}$ & $T^{* 2} \frac{\partial^{2} B^{*(3)}}{\partial T^{* 2}}$ \\
\hline 34.000 & $.7601451+00$ & .6503881-02 & $.1135932-03$ & $09908-05$ & $.1023184-01$ & $.7034165-02$ & 1346685 & $.1964124-04$ & $-.3878860-01$ & $.1493804-01$ & $-.7282659-03$ & $.7944414-04$ \\
\hline & & & & & -01 & -02 & 12 & & $318521-01$ & $8115-01$ & & \\
\hline 000 & $155+00$ & $2-02$ & $.9031570-04$ & $56404-05$ & $.1537457-01$ & $6210746-02$ & 58363 & $-.1400772-04$ & $-.2817998-01$ & $.1313663-01$ & $-.5746060-03$ & $.5650083-04$ \\
\hline 40.000 & $.7578706+00$ & $.5458663-02$ & $8127678-04$ & $.3990984-05$ & $.1754853-01$ & $-.5866018-02$ & $.1669489-03$ & $-.1199072-04$ & $-.2368243-01$ & $.1238601-01$ & $153853-03$ & $.4830714-04$ \\
\hline 42.000 & $.7569662+00$ & $.5180073-02$ & $.7353204-04$ & $.3447109-05$ & $-.1950848-01$ & $-.5556834-02$ & $.1508021-03$ & $-.1034466-04$ & $-.1961949-01$ & $.1171462-01$ & $-.4648615-03$ & $.4162992-04$ \\
\hline 44.000 & $.7560170+00$ & $.4928113-02$ & $.6684563-04$ & $.2998180-05$ & $.2128388-01$ & $-.5278010-02$ & $.1368907-03$ & $-.8987861-05$ & $-.1593147-01$ & $.1111067-01$ & $-.4214140-03$ & $.3613336-04$ \\
\hline & & & & & & & & & & & & -04 \\
\hline & & -02 & & & $-.2437423-01$ & $95282-02$ & -03 & $-.6912943-05$ & $-.9491460-02$ & $.1006853-01$ & $-.3509732-03$ & $.2774208-04$ \\
\hline 50.000 & .75 & 98793 & 4 & & $38-01$ & & $222-03$ & $-.6113269-05$ & $-.6664126-02$ & $.9615975-02$ & $-.3221983-03$ & $.2451341-04$ \\
\hline 52.000 & $.7519721+00$ & $.4122778-02$ & $.4751843-04$ & $.1818685-05$ & $-.2696987-01$ & $-.4392065-02$ & .96846 & -.543 & -.40580 & $.9201507-02$ & $-.2968225-03$ & $.2176903-04$ \\
\hline 54.000 & $.7509325+00$ & $.3960397-02$ & $.4400214-04$ & $.1624849-05$ & $.2811690-01$ & $.4214421-02$ & .895906 & $-.4850309-05$ & .1648514 & .8820553 & $-.2743313-03$ & .19421 \\
\hline 000 & & & & & & & & & & & & \\
\hline & & .36 & 14 & & : $5+2+3$ & & & & & & & $5239-04$ \\
\hline 60.000 & & 02 & & & 01 & & & & & & $.2203112-03$ & $.1413167-04$ \\
\hline 6 & .74 & $.3306762-02$ & $.3115717-04$ & $.9792535-06$ & $-.3272533-01$ & $3502828-02$ & $.6317619-04$ & -.29143 & .809939 & $.7301064-02$ & $-.1927095-03$ & $.1163595-04$ \\
\hline 68.000 & $34+\infty$ & .3101228 & -2 & .817 & 341 & $.3280247-02$ & .55 & 05 & 1 & .6827 & 03 & .96 \\
\hline & & & & & & & & & & & & \\
\hline 00 & .73 & .2 & 4 & 06 & & & & & & & & -05 \\
\hline 80.000 & & & & & & & & & & & -03 & $.5956980-05$ \\
\hline 84.000 & .73 & -02 & & & 01 & $1-02$ & .36 & -.129433 & & & & $.5148001-05$ \\
\hline 88.000 & .73 & .2 & 4 & & 1 & 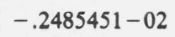 & .32 & 05 & .22 & & 03 & -05 \\
\hline & & & & & & & & & & & & \\
\hline & & & 34 & & 01 & & & & & & 04 & -05 \\
\hline 10 & .72 & .2 & & & 01 & & & & & & & \\
\hline & & $.1962811-02$ & $1141401-04$ & $370-06$ & $-.419900 /-01$ & $-.2000140-02$ & $.2305218-04$ & $-.060840 /-$ & .28 & .4250475 & -04 & \\
\hline 110.000 & .725 & .1 & 4 & & 01 & 02 & .2 & 06 & & & & \\
\hline & & & & & & & & & & & & \\
\hline & & & & & 01 & & & 06 & & & $3-04$ & $7-05$ \\
\hline 125.000 & .71 & $71-02$ & & & A & & & & & & & \\
\hline & & .15 & | & $.1200706-06$ & $-.4453033-01$ & $-.1640457-02$ & $.1497486-04$ & $-.3541623-06$ & $.3417275-01$ & $.3377858-02$ & $-.4516070-04$ & $.1402098-05$ \\
\hline I. & $.7160885+00$ & & $3=05$ & & -.4 & 02 & & -.3 & & & - . & \\
\hline & & & & & & & & & & & & \\
\hline & & & & & 1 & & & & 01 & & & $.1014236-05$ \\
\hline & & & & & $-4,4,-1$ & & & & & & & $2-06$ \\
\hline & & $.1263753-02$ & | & $.6511676-07$ & $-.4643022-01$ & $-.1317316-02$ & $.9860673-05$ & $-.1917290-06$ & $.3867643-01$ & $.2705922-02$ & $-.2966970-04$ & $.7577556-06$ \\
\hline 17 & $.7054931+00$ & $.1186388-02$ & 207214 \& 05 & $.5447388-07$ & (1<070<0 & $1025015=$ & & $.1603179-06$ & & & & \\
\hline & & & & & & & & & & & & .5 \\
\hline & $.7002375+00$ & $.1056672-02$ & & & & & & & & & & \\
\hline & .69 & .10 & $.3161315-05$ & & -.4 & & & & & & & $4-06$ \\
\hline & & $49-03$ & $.2868344-05$ & $.2926219-07$ & $-.4815079-0$ & $-.9897533-03$ & $.5716245-05$ & $-.8599488-07$ & $.4313016-01$ & $.2027781-02$ & $-.1715728-04$ & $.3392526-06$ \\
\hline 220.000 & .6 & .90 & & & 1 & & & & & & & \\
\hline 240.000 & & .0200920 & 200 & .197 & -.40 & -.000041 & & 07 & & & 04 & $.2288204-06$ \\
\hline & $.6850603+00$ & & & & & & $9847-05$ & & & & $7739-04$ & \\
\hline & .68 & .70 & $.1617818-05$ & .12 & -.492 & -.1 & & -.3 & & .14 &.- & $3120-06$ \\
\hline & $.6780125+00$ & $.6576224-03$ & $.1410465-05$ & $.1026944-07$ & $.4944242-01$ & $-.6815674-03$ & $.2803397-05$ & $-.3012122-07$ & $.4706704-01$ & $.1392697-02$ & $-.8393361-05$ & $.1186100-06$ \\
\hline
\end{tabular}




\section{References}

[1] Wigner, E., Phys. Rev. 40, 749 (1932); Uhlenbeck, G. E., and Gropper, L., Phys. Rev. 41, 79 (1932); Kirkwood. J. G., Phys. Rev. 44, 31 (1933).

[2] Kihara. T., Midzuno. Y., and Shizume, T., J. Phys. Soc. Japan 10, 249 (1955); Kihara. T., Revs. Mod. Phys. 27, 412 (1955).

[3] Boyd, M. E., Larsen, S. Y., and Kilpatrick, J. E., J. Chem. Phys. 50, 4034 (1969).

[4] Lennard-Jones. J. E., Proc. Roy. Soc. (London). A106, 463 (1924).

[5] Epstein, L. F., and Hibbert, C. J., J. Chem. Phys. 20, 752 (1952).
[6] Hirshfelder, J. O., Curtiss, C. F., and Bird, R. B., Molecular Theory of Gases and Liquids, (John Wiley and Sons, Inc.. New York, 1954.) Appendix I-B, p. 1114.

[7] Michels, H. H., Phys. of Fluids 9, 1352 (1966).

[8] Saxena, S. C., and Joshi, K. M., Ind. J. Phys. 36, 422 (1962).

[9] Sze, M., and Hsu, H., J. Chem. and Engr. Data 11, 77 (1966). An errata to this paper is to be published in the same journal in the near future.

[10] Sherwood, A. E., and Prausnitz, J. M., J. Chem. Phys. 41, 429 (1964).

(Paper 75Al-650) 



\section{Publications of the National Bureau of Standards*}

\section{Selected Abstracts}

Blaine, R. L., Tomes, L. A., Exploratory studies of early strength development in portland cement pastes and mortars, Nat. Bur. Stand. (U.S.), Bldg. Sci. Ser. 28, 14 pages (July 1970) 25 cents, SD Catalog No. C13.29/2:28.

Key words: Cement: cement mortar; cement paste; early strength; false set; hardening of cement; hydration; shear resistance; theory of cement hardening; time of set; vane-shear apparatus.

A modified vane-shear apparatus was used to measure the shear resistance of neat cement pastes of normal consistency and 1:2.75 (cement to sand) mortars of standard consistency, and to measure the increase in shear resistance with time as the cements hardened. The hardening process appeared to occur in three stages. The rate of increase of shear resistance as well as the duration of the different phases differed with the different cements. The results were analyzed in terms of the various theories proposed to explain the hardening of cements.

Brauer, G. M., Pyrolysis-gas chromatographic techniques for polymer identification, Chapter 2 . Thermal Characteristics Techniques in Techniques and Methods of Polymer Evaluations, P. Slade, Ed., II, 41-105 (Marcel Dehitier Publ. New York, N.Y., 1970).

Key words: Chromatographic analysis of degradation products; Polymer characterization; polymer identification; pyrolysis-gas chromatography of polymers; pyrolytic techniques.

Pyrolytic techniques used in conjunction with gas chromatography are very useful for elucidating the structure of macromolecules. These methods are a powerful tool in the qualitative characterization of the gross structure, the study of the thermal stability of polymers and the identification of their pyrolysis products, but have also found an increasing number of applications for the quantitative analysis of copolymeric systems and in studies of the kinetics of polymer degradation. The shape of the pyrograms is dependent on the structural characteristics such as the degree of branching or crosslinking, stereoregularity, crystallinity and monomer sequence length distribution in block and graft copolymers. Thus the pyrolysisgas chromatographic technique opens up new avenues in studying the ultimate arrangement of monomeric units within the polymer chain.

Bryson, J. O., Carpenter, E. F., Flexural behavior of prestressed concrete conipositt Tee-beams, Nat. Bur. Stand. (U.S.), Bldg. Sci. Ser. 31, 14 pages (July 1970) 25 cents, SD Catalog No. C13.29| 2:31. Catalog of standard reference materials, Nat. Bur. Stand. (U.S.), Spec. Pub. 260-1970 ed., 84 pages (July 1970) 75 cents, SD Catalog No. C13.10:260-1970 ed.

Key words: Composite concrete construction; prestressed concrete beams; Tee-beams.

Prestressed Tee-beams constructed by the split-beam method were tested to failure in flexure to study the behavior and ultimate strength of these beams and to compare their flexural characteristics with with those of prestressed beams of conventional construction. The compressive portion of the cross section of the split-beam is cast after the web of the beam has been formed and prestressed. The variables in the study included the percentage of prestressing steel, strength of concrete in the compressive element of the com- posite split-beams, manner of prestressing and web reinforcement. Results showed that the composite split-beams behaved similarly to the monolithically constructed beams on the basis of flexural response and ultimate load. The strength of the concrete for the compressive element can be reduced within limits from that required for the prestressed element without sacrificing ultimate load capacity. The required percentage of reinforcing steel is less for the split-beam compared with conventional beams.

Diller, D. E., Roder, H. M., Thermal conductivity measurements on fluid hydrogen at 17 to $200{ }^{\circ} \mathrm{K}$ and pressures to $10 \mathrm{MN} \mathrm{m}^{2}$ (Proc. 1969 Cryogenic Engineering Conf., June 16-18, 1969, Univ. of California, Los Angeles, Calif.), Chapter in Advances in Cryogenic Engineering 15, Paper No. C-1, 58-64 (Plenum Press, Inc., New York, N.Y., 1970).

Key words: Critical region; parahydrogen; thermal conductivity.

New absolute thermal conductivity measurements on fluid normal and parahydrogen are reported. A nominal accuracy of $2 \%$ has been achieved using a parallel plate apparatus in the temperature range 17 to $200 \mathrm{~K}$ at pressures to $100 \mathrm{~atm}$ and densities to 2.6 times critical. A graphical presentation of the data is given along isotherms, isochores, and isobars in the following regions: 1) the dilute gas, 2) the moderately dense gas, 3) the saturated and compressed liquid, 4) the critical region. Several interesting features of the behavior of the thermal conductivity of this fluid are pointed out: 1) the thermal conductivity of saturated liquid hydrogen increases with temperature between the triple point and the normal boiling point in contrast to the temperature dependence for most liquids other than helium, 2) a large anomalous increase in the thermal conductivity of hydrogen was found in the vicinity of the critical point.

Gonano, R., Adams, E. D., In Situ vapor pressure measurement for low temperature thermometry, Rev. Sci. Instr. 41, No. 5 , 716-719 (May 1970).

Key words: Capacitance manometer; cryogenic thermometry; manometry; pressure measurement; thermomolecular pressure difference; vapor pressure.

By use of a capacitance diaphragm manometer operated at cryogenic temperature, it is possible to measure the vapor pressure of cryogenic liquids without the uncertainties introduced by thermomolecular pressure gradients (thermal transpiration). Since the zero of this manometer is more stable at low temperature than at room temperature, useful sensitivity is also increased. Because of the increased accuracy at low pressure as well as great convenience and simplicity, this technique appears to have wide application in precise vapor pressure thermometry and similar work.

Hamilton, W. C., Edmonds, J. W., Tippe, A., Rush, J. J., Methyl group rotation and the low temperature transition in hexamethylbenzene. A neutron diffraction study, Discussions Faraday Soc., No. 48, 192-204 (1969).

Key words: Barrier to rotation; crystal; crystal structure; hexamethylbenzene; hindered rotation; phase transition; point methyl group; torsional oscillation.

Neutron diffraction studies of single crystals of hexamethylbenzene at $298^{\circ} \mathrm{K}$ and at $130^{\circ} \mathrm{K}$ indicate that the molecule in Phase II has approximate D ${ }_{d}$ symmetry. The amplitudes of libration of the methyl 
group and of rigid body motions of the molecule are consistent with earlier data, except that the barrier to methyl group rotation appears to be somewhat lower - in the neighborhood of $2000 \mathrm{~J} / \mathrm{mol}$.

Consideration of intra- and inter-molecular hydrogen atom contact distances and calculated potential energy curves using a 6-exp potential function suggest that intermolecular forces are important in determining the barrier to rotation of the methyl groups and that substantial changes in the intermolecular packing must be responsible for the lambda-point transition at $116^{\circ} \mathrm{K}$ and the consequent profound change in the potential barrier to internal rotation which has been previously observed.

Heinrich. K. F. J., Present state of the classical theory of quantitative electron probe microanalysis, Nat. Bur. Stand. (U.S.), Tech. Note 521,17 pages (Aug. 1970) 30 cents, SD Catalog No C13.46:521

Key words: Corrections; electron probe microanalysis; quantitative analysis; x-ray spectroscopy.

Although the foundations for a procedure of data reduction in quantitative electron probe analysis have not been changed for several years, there has been progress in the choice of expressions, parameters, and constants. A brief account of recommended expressions and procedures is given. Reference is made to the Standard Reference Materials of $\mathrm{Au}-\mathrm{Ag}$ and $\mathrm{Au}-\mathrm{Cu}$ alloys issued for electron probe microanalysis. These are especially useful for investigating the application of correction procedures.

Madey, T. E., Yates, J. T., Jr., Chemisorption on single crystals: $\mathrm{H}_{2}$ on (100) tungsten, Proc. Colloque Intern. sur la Structure et les Proprietes des Surfaces des Solides, Paris, France, July 7-11, 1969, No. 187, pp. 155-162 (Centre National de la Recherche Scientifique, Paris, France, 1970).

Key words: Binding state; chemisorption; hydrogen; isotopes; single crystal; tungsten; work function.

An ultrahigh vacuum apparatus has been constructed to study flash desorption of gaseous molecules from macroscopic single crystals. The crystals are in the form of thin discs, polished on both faces, whose total surface area is 95 percent of one orientation. A focussed light source external to the vacuum system is used to heat the nearly adiabatically suspended sample to $T>1200 \mathrm{~K}$; temperature inhomogeneities and end effects which complicate kinetic measurements on resistively heated samples are not present here. The sample is cleaned by electron bombardment prior to making adsorption measurements. Work function changes upon adsorption are monitored using an electron gun; a quadrupole mass spectrometer is used as the detector of desorbed species.

The chemisorption of $\mathrm{H}_{2}$ and $\mathrm{D}_{2}$ on a (100) oriented tungsten disc has been examined with this apparatus. The hydrogen desorption spectra reveal that two binding states exist on the (100) surface. The first order $\beta_{1}$ state desorbs with an activation energy of $\sim 25 \mathrm{kcal} / \mathrm{mole}$ and at saturation coverage, is twice as densely populated as the $\beta_{2}$. state. The coadsorption of an equimolar mixture of $\mathrm{H}_{2}$ and $\mathrm{D}_{2}$ shows that both states are isotopically mixed upon desorption. The work function-coverage relation is linear over the entire coverage range; the dipole moment per atom is 0.15 Debye, independent of binding state. Several models of $\mathrm{H}_{2}$ chemisorption are discussed.

Pfeiffer, E. R., Schooley, J. F., Effect of stress on the superconducting transition temperature of $\mathrm{SrTiO}_{3}$, J. Low Temp. Phys. 2, No.3/4,333-352 (August 7, 1970).

Key words: Anisotropic stress effect; hydrostatic pressure Nbdoped $\mathrm{SrTiO}_{3} ; 0-1.8$ kbars; reduced $\mathrm{SrTiO}_{3}$; superconductivity; transition temperatures; uniaxial compression.

The superconducting transition temperatures of several specimens of reduced $\mathrm{SrTiO}_{3}$ and of $\mathrm{Nb}$-doped $\mathrm{SrTiO}_{3}$ have been investigated as functions of hydrostatic and uniaxial compressive stresses up to 1.8 kbars. Large decreases in $T_{c}$ were observed in each specimen under hydrostatic pressure. Because of the low $T_{c}$ and small compressibility of $\mathrm{SrTiO}_{3}, \Delta\left(\ln T_{c}\right) / \Delta P$ and $\Delta\left(\ln T_{c}\right) / \Delta(\ln V)$ are orders of magnitude greater than the corresponding effects in the elemental superconductors. An anisotropic uniaxial stress effect was observed. [111] compression caused large decreases in $T_{c}$, while both small increases and small decreases in $T_{c}$ were observed for specimens under [100] compression. It is believed that the present results reveal a sensitive volume dependence of one or more of the parameters important to superconductivity in $\mathrm{SrTiO}_{3}$, and that no significant electron-transfer effects occurred in the range of stresses of this experiment.

Rockett, J. A., Objectives and pitfalls in the simulation of building fires with a computer, Fire Tech. 5, No. 4, 311-322 (November 1969).

Key words: Building; computer; fire; simulation.

The complex interactions between a building and a fire are being studied using the NBS computer facility. A description of the building in a form suitable for use with the computer has been developed. Obtaining sufficient detail in the description to allow a good representation of the building, with an easily manageable amount of input data, has been a major problem. In describing the fire, it is necessary to determine the ai movements induced by the fire, since these establish both the severity and extent of the fire, and the movement of smoke and toxic products. The technical problem is twofold; not enough is known about convective air movements and how to calculate them, and those calculations which can be carried out are excessively time consuming. Concurrently, information is lacking on the precise configuration of the building at the time of the fire; which doors are open or closed and where critical items are located. By highlighting the information needs for a successful calculation, the fire simulation study provides a guide to future research. Insofar as the study is able to represent correctly actual building fires, it provides a useful tool to the fire protection engineer.

Searles, S. K., Sieck, L. W., High pressure photoionization mass spectrometry, III. Reactions of $\mathbf{N O}^{+}\left(\mathbf{X}^{\prime} \Sigma^{+}\right)$with $\mathbf{C}_{3}-\mathbf{C}_{7}$ hydrocarbons at thermal kinetic energies, J. Chem. Phys. 53, No. 2, 794-797 (July 15, 1970).

Key words: Alkanes; excited states; ion-molecule reactions; mass spectrometry; photoionization; rate constants.

The vapor phase reaction of $\mathrm{NO}^{+}\left(\mathrm{X}^{\prime} \Sigma^{+}\right)$with $\mathrm{C}_{3}$ through $\mathrm{C}_{6}$ normal, branched, or cyclic alkanes was found to proceed exclusively via an $\mathrm{H}^{-}$transfer mechanism:

$$
\mathrm{NO}^{+}\left(\mathrm{X}^{1} \Sigma^{+}\right)+\mathrm{RH}_{2}-\mathrm{RH}^{+}+\mathrm{HNO}
$$

In addition to (I), $\mathrm{C}_{4} \mathrm{H}_{9}^{+}$was also formed by a second order process in the reaction with 3-methylhexane. Absolute rate constants were determined for all systems at thermal kinetic energies. Isomers containing tertiary $\mathrm{H}$ atoms were found to be the most reactive, exhibiting rate constants on the order of $10^{-9} \mathrm{~cm}^{3} /$ molecule-second. Isotopic labeling has verified that only the tertiary site is involved in the $\mathrm{H}^{-}$transfer reaction in those molecules having both secondary and tertiary $\mathrm{H}$ atoms. The rate constants found for $n$-alkanes and non-substituted cycloalkanes fall in the range $10^{-12}$ to $10^{-10} \mathrm{~cm}^{3}$ / molecule-second. The bimolecular reaction cyclo- $\mathrm{C}_{6} \mathrm{H}^{+}{ }_{11}+\mathrm{NO}$ $-\mathrm{C}_{6} \mathrm{H}_{11} \mathrm{NO}^{+}$was also noted at higher pressures. No further reaction of the $\mathrm{RH}^{+}$species generated in (I) was found in any other $\mathrm{RH}_{2}-\mathrm{NO}$ combination at pressures up to 0.5 torr.

Shinyayev, A. Y., Butrymowicz, D. B., Interdiffusion in and the phase diagram for vanadium-rich alloys of the $\mathrm{V}$-Al system at pressures 0 to 47 kbar, Met. Trans. 1, No. 7, 1905-1907 (July 1970) Simson, B. G., Mandel, J., Brenner, F. C., Research for a uniform quality grading system for tires. III, Breaking energy Rubber Chem. and Tech. 43, No. 2, 356-369 (March 1970).

Key words: Alloys; aluminum; diffusion; high-pressure; phasediagram; vanadium.

The $\mathrm{V}-\mathrm{Al}$ system between 16 and 39 at. pct. $\mathrm{Al}$ was studied at $1400{ }^{\circ} \mathrm{C}$ under pressures of 0,30 , and 47 kbar. Electron microprobe analysis, x-ray diffraction, microhardness readings, and metallographic examination revealed only a single solid solution. 
Interdiffusion coefficients were determined as a function of composition and pressure. Concentration gradients were measured with an electron microprobe analyzer and the diffusion coefficients were calculated by the Matano analysis. At $1400{ }^{\circ} \mathrm{C}$, the value of the interdiffusion coefficient varies from 1 to $12 \times 10^{-9} \mathrm{~cm}^{2} / \mathrm{s}$, increasing with aluminum content and decreasing with increasing pressure

Straty, G. D., Prydz, R., The vapor pressure of liquid fluorine, (Proc. 1969 Cryogenic Engineering Conf., June 16-18, 1969, Univ. of California, Los Angeles, Calif.), Chapter in Advances in Cryogenic Engineering 15, Paper No. B-1, 36-4l (Plenum Press, Inc., New York, N.Y., 1970).

Key words: Critical point pressure; fluorine; normal boiling point; vapor pressure data; vapor pressure equation.

This paper reports new vapor pressure measurements on liquid fluorine from the triple point to the critical point at $1 \mathrm{~K}$ intervals. Accuracies in both temperature and pressure approach a few hundredths of a percent. The data were fitted to a newly developed. non-analytic vapor pressure equation and compared graphically to earlier, inconsistent measurements available in the literature. Of this earlier data, the estimated $P-T$ values above the normal boiling point and the value given for the critical pressure are in error by as much as 10 to 15 percent.

Sugar, J., Spectrum of doubly ionized thulium (Tm II), J. Opt. Soc. Am. 69, No. 4, 454-466 (April 1970).

Key words: Doubly ionized thulium; energy levels of Tm III; radial energy integrals of Tm III; spectrum of Tm III.

A list of 848 low excitation spectral lines of Tm III has been obtained by utilizing the sliding spark light source at a peak current of $6 \mathrm{~A}$. An analysis of these lines yielded 108 energy levels belonging to the configurations $4 f^{13}, 4 f^{12} 5 d, 4 f^{12} 6 s$, and $4 f^{12} 6 p$. The classified lines and level values are presented as well as a theoretical interpretation of these configurations.

Tighe, N. J., Hockey, B. J., Ion thinning of electron microscope specimens (Proc. 10th Symp. Electron, Ion and Laser Beam Tech., May 21-23, 1969. National Bureau of Standards, Gaithersburg, Md.), Record of 10th Symposium on Electron, Ion, and Laser Beam Technology, L. Marton, Ed., pp. 375-380 (San Francisco Press Inc., San Francisco, Calif., 1970).

Key words: $\mathrm{Al}_{2} \mathrm{O}_{3}$; argon ion bombardment; ceramics; electron microscopy; hardness; ion thinning; $\mathrm{SiO}_{2}$.

In recent years, it has been possible to prepare ceramic specimens for transmission electron microscopy using ion bombardment for thinning bulk samples to less than $\sim 0.5 \mu \mathrm{m}$ thickness. Chemical inertness and electrical insulating properties of many ceramic materials make chemical and electrochemical methods, such as those used for metals, difficult to apply. Even when suitable chemical polishes are found they often are useful only for certain crystallographeric orientations and not at all useful for polycrystalline bodies. In this laboratory, the ion-thinning technique has been applied to polycrystalline and single-crystalline ceramics including alumina, magnesia, silica and zirconia.

Prior to ion thinning, disk specimens 2.3 or $3 \mathrm{~mm}$ in diameter are cut from thin slices $(40$ to $150 \mu \mathrm{m})$ of the selected samples. When a specimen is particularly fragile because of large deformation, fractures, or porosity, a supporting rim is cemented to it. Specimens are thinned simultaneously from both sides and are rotated during thinning to eliminate or reduce directional grooving. They are thinned with argon ion beams using accelerating voltages of 4 to $8 \mathrm{kV}$ and beam currents of 50 to $150 \mu \mathrm{A}$. Thinning rates vary from $1 / 2$ to $4 \mu \mathrm{m} / \mathrm{hr}$ depending on the specimen material and on the operating conditions.

The ion-thinned specimens have electron transparent regions which usually are more extensive than those in the best chemically thinned single crystal specimens. Studies of the microstructure of hotpressed alumina, periclase brick, quartz and magnetite rocks and a number of sintered ceramics have been undertaken. The dislocation substructure, impurity precipitates, second phase grains, and voids have been revealed by transmission electron microscopy; and, in some cases, the defects have been related to the deformation sustained by specimens during forming or during mechanical testing. Because specimens can be thinned from only one side, it has been possible to study the damage in single and polycrystalline alumina specimens which is produced by hardness indents and by mechanical polishing with diamond abrasives.

Torrance, K. E., Orloff, L., Rockett, J. A., Numerical study of natural convection in an enclosure with localized heating from below-creeping flow to the onset of laminar instability, J. Fluid Mech. 36, Part I, 33-54 (June 1969).

Key words: Circular cylinder; enclosures; natural convection; numerical.

An analytical study was made of the natural convection induced in an enclosure by a small hot spot centrally located on the floor. The enclosure was a circular cylinder, vertically oriented, with height equal to radius. A Prandtl number of 0.7 (air) was assumed; the Grashof number (Gr) was based on cylinder height and hot spot temperature. The equations of fluid flow in axisymmetric cylindrical coordinates were simplified with the Boussinesq approximation. The equations were solved numerically with a computationally stable, explicit method. The computation, starting from quiescent conditions, proceeded through the initial transient to the fully developed flow. Solutions were obtained for Gr from $4 \times 10^{4}$ to $4 \times 10^{10}$. The calculated flows were compared with the experimental flows presented in a companion paper, Torrance, Orloff and Rockett (1968). The experimental flows were laminar for $\mathrm{Cr} \leq 1.2 \times 10^{9}$; turbulence was observed above this value. In the laminar flow region $\left(\mathrm{Gr} \lesssim 1.2 \times 10^{9}\right)$ agreement between the theoretical and experimental flow patterns was excellent. When extended into the experimentally observed turbulent range $\left(\mathrm{Gr}=4 \times 10^{10}\right)$ the theoretical flow developed a periodic vortex shedding, suggestive of the onset of transition.

Verdier, P. H., Relaxation behavior of the freely jointed chain, J. Chem. Phys. 52, No. 11, 5512-5517 (June 1, 1970).

Key words: Chain dynamics; polymers; random walk; relaxation; stochastic process.

A method is presented for treating the relaxation behavior of the freely jointed chain model of a random coil polymer. Exact results are exhibited for the relaxation of quantities linear in chain coordinates. For the treatment of quantities quadratic in chain coordinates, a numerical approach is employed and exemplified by obtaining the autocorrelation in the square of end-to-end length for chains of up to 16 beads. In both cases, the rapid approach of the behavior of the freely jointed chain of $N$ beads to that of the Rouse model of $N$ statistical segments is demonstrated.

Winogradoff, N. N., Neill, A. H., Jr., Temperature dependence of the power output of the spontaneous emission from $\mathrm{GaAs}$ laser diodes, Intern. J. Electron. 28, No. 5, 401-406 (1970).

Key words: Band tailing: GaAs lasers; non-radiative recombination centers; radiative recombination; temperature dependence; valence band.

The spectra of the spontaneous emission from GaAs laser diodes fabricated by vapor phase epitaxy and containing a high degree of compensation in the $p$-type side of the junction are, in many respects, similar to those previously reported for the cathodoluminescence of homogeneous $p$-type material. The temperature dependence of the spectra of these diodes supports a model where the emission is attributed to radiative transitions between a narrow band of states near the conduction band edge and an exponential distribution of states extending the valence band into the forbidden gap.

An increase in temperature then results in an increase or a decrease in the radiative power output depending on the position of the quasi Fermi level for electrons relative to the above narrow band of states and a distribution of non-radiative levels below it. 
Younglove, B. A., Measurements of the dielectric constant of saturated liquid oxygen (Proc. 1969 Cryogenic Engineering Conf., June 16-18, 1969, Univ. of California, Los Angeles, Calif.), Chapter in Advances in Cryogenic Engineering 15, Paper No. C-3, 70-75 (Plenum Press, Inc., New York, N.Y., 1970).

Key words: Clausius-Mossotti function; dielectric constant; oxygen; polarizability; saturated liquid.

Dielectric constant measurements of saturated liquid oxygen show a variation of 1.22 near the critical point to 1.57 near the triple point. The calculation "polarizability" (Clausius-Mossotti function) shows a variation of $1.3 \%$, with the maximum occurring at about $150 \mathrm{~K}$. The decrease in polarizability near critical is attributed to inaccuracies in the densities in this region. The uncertainty in dielectric constant is about $0.01 \%$.

\section{Other NBS Publications}

J. Res. Nat. Bur. Stand. (U.S.), 74B (Math. Sci.), No. 4 (October-December 1970), SD Catalog No. C13.22/ sec. B: 74/4. $\$ 1.25$

A property of the triangle groups. J. Lehner.

Some theorems on tensor composite graphs. M. F. Capobianco.

The diophantine approximation of roots of positive integers. C. F. Osgood.

The minimum number of problems to cover all subproblems. H. J. Greenberg.

Error estimates for the solution of linear algebraic systems. Brother K. E. Fitzgerald, F.S.C.

On the singular values of a product matrices. W. Watkins.

Contractifiable semigroups. P. R. Meyers.

Publications of the National Bureau of Standards.

J. Res. Nat. Bur. Stand. (U.S.), 74C (Engr. and Instr.) Nos. 3 \& 4 (July-Dec. 1970), SD Catalog Number C13.22/sec. C: $74 / 3 \& 4$. $\$ 1.25$.

Techniques for comparing four-terminal-pair admittance standards. R. D. Cutkosky.

Some applications for series impedance elements in radio frequency immittance measurements. L. E. Huntley.

A pulse heating method for the measurement of melting point of electrical conductors (thin wires) above $2000 \mathrm{~K}$. A. Cezairliyan.

A time-shared computer system for diffractometer control. H. A. Alperin and E. Prince.

Tensile deformation of vapor-deposited copper reinforced with tungsten wires. W. D. Jenkins, W. A. Willard, and D. A. Harne.

"Connector-pair" techniques for the accurate measurement of two-terminal low-value capacitances. A. Millea.

The use of dew-point temperature in humidity calculations. L. A. Wood.

Bolovac application for $\mathrm{HF}$ and microwave power measurement and standardization. M. C. Selby.

Arp, V., Heat transport through helium II, Cryogenics 10, No. 2. 96-105 (April 1970)

Bridges, J. M., Wiese, W. L., The oscillator-strength scale for Fe I, Astrophys. J. 161, L71-L75 (July 1970).

Brower, W. S., Jr., Fang, P. H., Dielectric constants of scheelite structure crystals, J. Appl. Phys. 40, No. 12, 4988-4989 (November 1969).

Brower, W. S., Jr., Fang, P. H., Dielectric constants of zinc tungstate, J. Appl. Phys. 41, No. 5, 2266 (April 1970).

Brower, W. S., Jr., Farabaugh, E. N., Dislocation etchant for single crystal $\mathrm{Y}_{2} \mathrm{O}_{3}$, J. Am. Ceram. Soc. 53, No. 4, 225 (April 1970).

Cezairliyan, A., High-speed methods of measuring specific heat of electrical conductors at high temperatures (A review), High Temperatures - High Pressures 1, 517-529 (1969).

Childs, G. E., Diller, D. E., Refractive index of liquid deuterium, (Proc. 1969 Cryogenic Engineering Conf., June 16-18, 1969, Univ. of California, Los Angeles, Calif.), Chapter in Advances in
Cryogenic Engineering 15, Paper No. C-2, 65-69 (Plenum Press, Inc., New York, N.Y., 1970).

Clark, A. F., Childs, G. E., Wallace, G. H., Low-temperature electrical resistivity of some engineering alloys (Proc. 1969 Cryogenic Engineering Conf., June 16-18, 1969, Univ. of California, Los Angeles, Calif.), Chapter in Advances in Cryogenic Engineering 15, Paper No. C-5, 85-90 (Plenum Press, Inc., New York, N.Y., 1970).

Daney, D. E., Rapial, A. S., Preparation and characterization of slush hydrogen and nitrogen gels (Proc. 1969 Cryogenic Engineering Conf., June 16-18, 1969, Univ. of California, Los Angeles, Calif.), Chapter in Advances in Cryogenic Engineering 15, Paper No. L-4, 467-475 (Plenum Press, Inc., New York, N.Y., 1970). Duncan, A. G., Hiza, M. J., A multipurpose phase equilibrium apparatus to study mixtures of cryogenic fluids: application to argon-methane (Proc. 1969 Cryogenic Engineering Conf., June 16-18, 1969, Univ. of California, Los Angeles, Calif.), Chapter in Advances in Cryogenic Engineering 15, Paper No. B-2, 42-45 (Plenum Press, Inc., New York, N.Y., 1970).

Geil, G. W., Feinberg, I. J., Temperature changes in specimens in microplasticity tests, Met. Trans. 1, No. 7, 1845-1851 (July 1970).

Giacchetti, A., Stanley, R. W., Zalubas, R., Proposed secondary standard wavelengths in the spectrum of thorium, J. Opt. Soc. Am. 69, No. 4, 474-489 (April 1970).

Hanson, D. W., Quasi-optical components using total reflection in dielectrics, IEEE Trans. Microwave Theory Tech. MTT-18, No. 4, 233-234 (April 1970).

Hendricks, R. C., Simoneau, R. J., Smith, R. V., Survey of heat transfer to near-critical fluids (Proc. 1969 Cryogenic Engineering Conf., June 16-18, 1969, Univ. of California, Los Angeles, Calif.), Chapter in Advances in Cryogenic Engineering 15, Paper No. G-1, 197-237 (Plenum Press, Inc., New York, N.Y., 1970).

Heydemann, P. L., Ultrasonic measurements at pressures up to 50 kbar, Proc. Intern. Collog. Solids at High Pressures, Grenoble, France, Sept. 8, 1969, No. 188, pp. 461-467 (Centre National de la Recherche Scientifique, Paris, France, 1970).

Hiza, M. J., Solid-vapour equilibria research on systems of interest in cryogenics, Cryogenics 10, No. 2, 106-115 (April 1970).

Kidnay, A. J., Hiza, M. J., Dickson, P. F., Adsorption kinetics in a ternary system containing hydrogen (Proc. 1969 Cryogenic Engineering Conf., June 16-18, 1969, Univ. of California, Los Angeles, Calif.), Chapter in Advances in Cryogenic Engineering 15, Paper No. B-3, 46-49 (Plenum Press, Inc., New York, N.Y., 1970).

LaFleur, P. D., Editor, Activation analysis section: Summary of activities July 1968 to June 1969, Nat. Bur. Stand. (U.S.) Tech. Note 508, 147 pages (July 1970) \$1.25, SD Catalog No. C13.46:508.

Levin, E. M., X-ray determination of the thermal expansion of silver nitrate, J. Am. Ceram. Soc. 52, No. 1, 53-54 (January 1969).

Locke, J. W., Penn, R., Rick, J., Bunten, E., Hare, G., Compilation and use of criminal court data in relation to Pre-Trial Release of defendants: pilot study, Nat. Bur. Stand. (U.S.), Tech. Note 535 , 242 pages (Aug. 1970) \$1.75, SD Catalog No. C13.46:535.

Lutz, G. J., Masters, L. W., Determination of carbon in high purity metals by photon activation analysis. Anal. Chem. 42, No. 8, 948-950 (July 1970).

Marvin. R. S., Rheological Models and measurements (Proc. 5th. Intern. Congress on Rheology, Kyoto, Japan, Oct. 7-11, 1968), Chapter in Fifth International Congress on Rheology, S. Onogi, Ed., 1, 85-96, University of Tokyo Press and University Park Press, Tokyo, Japan, 1969)

Mauvais, C. J., Latanision, R. M., Ruff, A. W., Jr., On the anisotropy observed during the passivation of nickel monocrystals, J. Electrochem. Soc. 117. No. 7, 902-903 (July 1970).

McNish, A. G., Progress on the Metric Study Program, Proc. Conf. 18th Annual Meeting of Standards Engineers Society, Washington, D.C., Sept. 17-19, 1969, pp. 53-56 (Standards Engineers Society, Philadelphia, Pa., 1969).

McNish, A. G., The impact of increasing metric usage, Proc. Conf. Industrial Heating Equipment Association, Spring (77th Consecutive) Meeting, Hot Springs, Virginia, May 19-21, 1969, Exhibit E (Industrial Heating Equipment Assoc., Washington, D.C., 1969).

Melmed, A. J., Carroll, J. J., Ellipsometry, LEED, and FEM study of evaporated epitaxial films of iron on (011) tungsten, Surface Sci. Letters to the Editor 19, No. 1, 243-248 (January 1970). 
Mighell, A. D., Reimann, C. W., Santoro, A., The crystal structure of the 2:1 dimer [ (pyridine $\mathrm{N}$-oxide $\left.)_{2} \mathrm{CuBr}_{2}\right]_{2}$, Chem. Commun, 4 , 204 (1970)

Molino, J. A., Noise the growing hazard, Dialog 1, No. 4, 26-28 (1970).

Negas, T., Roth, R. S., The system $\mathrm{SrMnO}_{3-x}$, J. Solid State Che 1, 409-418 (1970).

Page, C. H., Relations among systems of electromagnetic equations, Am. J. Phys. 38, No. 4, 421-424 (April 1970).

Page, C. H., The trolley problem, Am. J. Phys. 38, No. 5, 608-610 (May 1970).

Phillips, S. L., Johnson, C. E., Effect of atmospheric exposure on the contact resistance of selected tin alloys, J. Electrochem. Soc. 117, No. 6, 827-830 (June 1970).

'Reed, R. P., Durcholz, R. L., Cryostat and strain measurement for tensile tests to $1.5{ }^{\circ} \mathrm{K}$ (Proc. 1969 Cryogenic Engineering Conf., June 16-18, 1969, Univ, of California, Los Angeles, Calif.), Chapter in Advances in Cryogenic Engineering 15, Paper No. D-3, 109116 (Plenum Press, Inc., New York, N.Y., 1970).

Robertson, A. F., Gross, D., Fire load, fire severity, and fire endurance, Am. Soc. Testing Mater. ASTM Spec. Tech. Publ. 464, Fire Test Performance, pp. 3-29 (January 1970).

Searles, S. K., Sieck, L. W., Ausloos, P., Reactions of $\mathrm{C}_{2} \mathrm{H}_{6}^{+}$. Formation of the $\left(\mathrm{C}_{2} \mathrm{H}_{6}\right)_{2}^{+}$ion, J. Chem. Phys. 53, No. 2, 849-850 (July $15,1970)$

Sher, A. H., Nomographs for use in the fabrication and testing of Ge(Li) detectors, Nat. Bur. Stand. (U.S.), Tech. Note 537, 18 pages (Aug. 1970) 30 cents, SD Catalog No. C13.46:537.

Sindt, C. F., Ludtke, P. R., Slush hydrogen flow characteristics and solid fraction upgrading (Proc. 1969 Cryogenic Engineering Conf., June 16-18, 1969, Univ. of California, Los Angeles, Calif.), Chapter in Advances in Cryogenic Engineering 15, Paper No. I-6, 382-390 (Plenum Press, Inc., New York, N.Y., 1970).

Snyder, N. S., Heat transport through helium II: Kapitza conductance Cryogenics 10, No. 2, 89-95 (April 1970).

Stevenson, D. R., Technical Standards Coordinator, Heavy-duty alpha-cellulose-filled melamine tableware, Nat. Bur. Stand. (U.S.), Voluntary Prod. Stand. 25-70, 8 pages (Aug. 1970) 10 cents, SD Catalog No. C13.20/2:25-70. Supersedes Commercial Standard C. $173-50$.

Stevenson, D. R., Technical Standards Coordinator, Melamine dinnerware (alpha-cellulose-filled) for household use, Nat. Bur. Stand. (U.S.), Voluntary Prod. Stand. 24-70, 9 pages (Aug. 1970) 10 cents, SD Catalog No. C13.20/2:24-70. Supersedes Commercial Standard CS 210-57.

Steward, W. G., Smith, R. V., Brennan, J. A., Cooldown transients in cryogenic transfer lines (Proc. 1969 Cryogenic Engineering
Conf., June 16-18, 1969, Univ. of California, Los Angeles, Calif.), Chapter in Advances in Cryogenic Engineering 15, Paper No. I-2, 354-363 (Plenum Press, Inc., New York, N.Y., 1970).

Stief, L. J., Payne, W. A., DeCarlo, V. J., Gorden, R., Jr., Ausloos, P., Comment on "Xenon sensitized photolysis of carbon dioxide", J. Chem. Phys. 53, No. 1, 475-476 (July 1, 1970).

Straty, G. C., Prydz, R., Melting curve and triple-point properties of fluorine, Physics Letters 31 A, No. 6, 301-302 (March 23, 1970).

Sugar, J., Configuration $4 f^{13} 5 d$ of doubly ionized ytterbium, J. Opt. Soc. Am. 60, No. 4, 571-572 (April 1970).

Swartzendruber, L. J., Bennett, L. H., Clustering, cold work, and the Mössbauer effect doublet structure in $\mathrm{Cu}-\mathrm{Ni}-\mathrm{Fe}$ alloys, Physics Letters 31A, No. 10, 581-582 (May 18, 1970).

Torrance, K. E., Orloff, L., Rockett, J. A., Experiments on natural convection in enclosures with localized heating from below, J. Fluid Mech. 36, Part I, 21-31 (1969).

Weber, L. A., The P-V-T surface of oxygen in the critical region; densities of saturated liquid and vapor (Proc. 1969 Cryogenic Engineering Conf., June 16-18, 1969, Univ. of California, Los Angeles, Calif.), Chapter in Advances in Cryogenic Engineering 15, Paper No. B-4, 50-57 (Plenum Press, Inc., New York, N.Y., 1970).

Wegstein, J. H., Automated fingerprint identification, Nat. Bur. Stand. (U.S.), Tech. Note 538, 33 pages (Aug. 1970) 40 cents, SD Catalog No. C13.46:538.

White, H. J., Jr., Counties and county equivalents of the states of the United States, Nat. Bur. Stand. (U.S.), Fed. Info. Process. Stand. Publ. (FIPS Pub) 6-1, 34 pages (1970) 45 cents, SD Catalog No. C13.52:6-1. Supersedes FIPS PUB 6, Nov. 1, 1968.

White, H. J., Jr., States and outlying areas of the United States, Nat. Bur. Stand. (U.S.), Fed. Info. Process. Stand. Publ. (FIPS Pub) 5-1, 4 pages, (1970) 20 cents, SD Catalog No. C13.52:5-1. Supersedes FIPS PUB 5, Nov. 1, 1968.

Winogradoff, N. N., Neill, A. H., Petrescu-Prahova, J. B., Band tailing and the spontaneous spectra of compensated epitaxial GaAs laser junctions, IEEE J. Quantum Electron. Q.E. 6, No. 6, 305-310 (June 1970).

*Publications for which a price is indicated are available by purchase from the Superintendent of Documents, U.S. Government Printing Office, Washington, D.C. 20402 (foreign postage, one-fourth additional). The NBS non-periodical series are also available from the National Technical Information Service, Springfield, Va. 22151. Reprints from outside journals and the NBS Journal of Research may often be obtained directly from the authors. 